\title{
Methodological flaws in meta-analyses of clinical studies on the management of knee osteoarthritis with stem cells: a systematic review
}

\author{
Christoph Schmitz ${ }^{1^{*}}$, Christopher Alt ${ }^{2,3}$, David A. Pearce ${ }^{4,5,6}$, John P. Furia ${ }^{7}$, \\ Nicola Maffulli ${ }^{8,9,10}$, Eckhard U. Alt $t^{2,3,6,11}$ \\ 1 Institute of Anatomy, Faculty of Medicine, LMU Munich, Munich, Germany \\ 2 InGeneron, Inc., Houston, TX, USA \\ 3 IsarKlinikum, Munich, Germany \\ 4 Sanford Research, Sioux Falls, SD, USA \\ 5 Sanford Health, Sioux Falls, SD, USA \\ 6 Sanford School of Medicine, University of South Dakota, Sioux Falls, SD, USA \\ 7 SUN Orthopedics of Evangelical Community Hospital, Lewisburg, PA, USA \\ 8 Department of Musculoskeletal Disorders, Faculty of Medicine and Surgery, \\ University of Salerno, Salerno, Italy \\ 9 Centre for Sports and Exercise Medicine, Barts and The London School of \\ Medicine and Dentistry, Mile End Hospital, Queen Mary University of \\ London, London, England \\ ${ }^{10}$ School of Pharmacy and Bioengineering, Guy Hilton Research Centre, Keele \\ University School of Medicine, Stoke on Trent, England \\ ${ }^{11}$ Heart and Vascular Institute, Department of Medicine, Tulane University \\ Health Science Center, New Orleans, LA, USA
}

Background: Conclusions of meta-analyses of clinical studies may substantially influence opinions of perspective patients and stakeholders in health care. Nineteen meta-analyses of clinical studies on the management of primary knee osteoarthritis (pkOA) with stem cells, published between January 2020 and July 2021, came to inconsistent conclusions regarding the efficacy of this treatment modality. It is possible that a separate meta-analysis based on an independent, systematic assessment of clinical studies on the management of pkOA with stem cells may reach a different conclusion.

Methods: PubMed, Web of Science and the Cochrane library were systematically searched for clinical studies and meta-analyses of clinical studies on the management of pkOA with stem cells. All clinical studies and meta-analyses identified were evaluated in detail, as were all sub-analyses included in the meta-analyses.

Results: The inconsistent conclusions regarding the efficacy of treating pkOA with stem cells in the 19 assessed meta-analyses were most probably based on substantial differences in literature search strategies among different authors, misconceptions

Correspondence

Dr. Christoph Schmitz, M.D. Institute of Anatomy Faculty of Medicine LMU Munich D-80336 Munich Germany

Phone: +49-89-2180-72620

Fax: +49-89-2180-72683 E-mail: christoph_schmitz@med.unimuenchen.de about meta-analyses themselves, and misconceptions about the biology of stem cells. An independent, systematic review of the literature yielded a total of 183 studies, of which 33 were randomized clinical trials, including a total of 6860 patients with pkOA. However, it was not possible to perform a scientifically sound meta-analysis.

Conclusion: Clinicians should interpret the results of the 19 assessed meta-analyses of clinical studies on the management of pkOA with stem cells with caution, and should be cautious of the conclusions drawn therein. Clinicians and researchers should strive to participate in FDA and/or EMA reviewed and approved clinical trials to provide clinically and statistically valid efficacy.

Keywords: meta-analyses; primary knee osteoarthritis; stem cells; systematic review 


\begin{abstract}
Background: Conclusions of meta-analyses of clinical studies may substantially influence opinions of perspective patients and stakeholders in health care. Nineteen meta-analyses of clinical studies on the management of primary knee osteoarthritis (pkOA) with stem cells, published between January 2020 and July 2021, came to inconsistent conclusions regarding the efficacy of this treatment modality. It is possible that a separate meta-analysis based on an independent, systematic assessment of clinical studies on the management of pkOA with stem cells may reach a different conclusion.

Methods: PubMed, Web of Science and the Cochrane library were systematically searched for clinical studies and meta-analyses of clinical studies on the management of pkOA with stem cells. All clinical studies and meta-analyses identified were evaluated in detail, as were all sub-analyses included in the meta-analyses.

Results: The inconsistent conclusions regarding the efficacy of treating pkOA with stem cells in the 19 assessed meta-analyses were most probably based on substantial differences in literature search strategies among different authors, misconceptions about meta-analyses themselves, and misconceptions about the biology of stem cells. An independent, systematic review of the literature yielded a total of 183 studies, of which 33 were randomized clinical trials, including a total of 6860 patients with pkOA. However, it was not possible to perform a scientifically sound meta-analysis.

Conclusion: Clinicians should interpret the results of the 19 assessed meta-analyses of clinical studies on the management of pkOA with stem cells with caution, and should be cautious of the conclusions drawn therein. Clinicians and researchers should strive to participate in FDA and/or EMA reviewed and approved clinical trials to provide clinically and statistically valid efficacy.
\end{abstract}

Keywords: meta-analyses; primary knee osteoarthritis; stem cells; systematic review 


\section{Introduction}

Osteoarthritis (OA) is the most common cause of pain and disability worldwide, especially in the elderly population. ${ }^{1-3}$ The hip and knee joints are mostly commonly affected, and women are more frequently affected than men. ${ }^{4,5}$ There is no cure for OA despite its high prevalence and deleterious impact on the quality of life of affected individuals. ${ }^{1,3}$ The currently available therapeutic measures aim to relieve pain and maximize functional capacity and quality of life, while minimizing adverse effects from drugs and invasive interventions. ${ }^{6}$ Patients suffering from primary knee OA (pkOA) often report refractory, severe and disabling pain, and are eventually referred for partial or total arthroplasty. 7,8

Intra-articular (i.a.) injection of platelet rich plasma (PRP), corticosteroid (CS) and hyaluronic acid (HA) are commonly used to manage pkOA.9-11 These injections can easily be administered, fewer treatment sessions are necessary compared with other treatments, and treatment adherence of patients is relatively easy to achieve (except for repeated injections). According to the Osteoarthritis Research Society International (OARSI) guidelines, i.a. injection of CS and HA (but not of PRP) are among the recommended treatments for pkOA, dependent upon comorbidity status. ${ }^{12}$ A recent metaanalysis of the efficacy and safety of i.a. injection of CS and $\mathrm{HA}$ for pkOA concluded that both therapies are relatively safe and lead to comparable improvement of knee function. ${ }^{13}$ According to such meta-analysis, better short-term effects (up to 1 month) are obtained with CS than with HA, and better long-term effects (up to 6 months) with HA than with CS. ${ }^{13}$ On the other hand, i.a. injection of HA causes more topical adverse effects than i.a. injection of CS. ${ }^{13}$

During the last 2 decades, various studies proposed the management of pkOA with different types of stem cells. By means of a systematic literature search in PubMed, Web of Science and the Cochrane database according to the PRISMA (Preferred Reporting Items for Systematic Reviews and MetaAnalyses) guidelines ${ }^{14}$, we identified 19 meta-analyses, all published between January 2020 and July 2021. ${ }^{15-33}$ Unfortunately, the conclusions of these meta-analyes are inconsistent. Specifically, these meta-analyses demonstrated (i) efficacy of treating pkOA with stem cells; ${ }^{16,18,19,23,28,30}$ (ii) superiority of autologous stem cells derived from adipose tissue over other types of stem cells in the management of pkOA; ${ }^{15,20,22,27,32}$ (iii) efficacy of treating pkOA with autologous, adipose-derived stem cells, without comparison with other types of stem cells; ${ }^{21,33}$ (iv) superiority of autologous, bone marrow-derived stem cells over autologous, adipose-derived stem cells in the management of pkOA; ${ }^{31}(\mathrm{v})$ efficacy of treating pkOA with autologous, bone marrowderived stem cells, without comparison with other types of stem cells; ${ }^{17}$ (vi) efficacy of treating pkOA with allogeneic stem cells; ${ }^{25}$ (vii) efficacy of treating pkOA with stem cells only in conjunction with surgery; ${ }^{29}$ and (viii) lack of efficacy of treating pkOA with stem cells ${ }^{24,26}$ (Table S1) (Tables S1S22 are provided in the Online Supplementary Material).

To determine the reasons for these inconsistent conclusions, we performed a comprehensive assessment of all clinical studies included in these 19 meta-analyses. ${ }^{15-33}$ Furthermore, we determined whether a separate meta-analysis based on an independent, systematic assessment of studies on the management of pkOA with stem cells would reach a different conclusion.

\section{Methods}

2.1. Literature search

PubMed, Web of Science and the Cochrane library were searched for "knee osteoarthritis stem cell*", "knee osteoarthritis stromal vascular fraction" and "knee osteoarthritis SVF" from the days of inception of these databases until August 07, 2021 according to the PRISMA (Preferred Reporting Items for Systematic Reviews and MetaAnalyses)14 guidelines. Duplicates were excluded. This search strategy found many more clinical studies than evaluated in the 19 assessed meta-analyses. ${ }^{15-33}$

\subsection{Assessment of published meta-analyses}

The stratetgy of the first assessment of the identified publications is summarized in Figure 1. In a first step, for each identified publication it was determined by reading title and abstract whether it represented a meta-analysis of clinical studies on the management of pkOA with stem cells and was published in 2020 or 2021. This was independently undertaken by CS and CA. Results were compared and discussed until agreement was achieved. Afterwards, all studies included in the identified meta-analyses that reported the management of pkOA with stem cells were classified with regard to the type of study as summarized in Tables S2 and $\mathrm{S} 3$, as well as with regard to the type of stem cells used as summarized in Table S4. Thereafter, all identified metaanalyses were classified by CS and CA with regard to the type of meta-analysis performed as summarized in Table S5.

Then, 2 types of meta-analyses were excluded from further assessment: those in which only endpoints of the same patients before and after treatment were compared (four metaanalyses) (Class 2 in Table S5), and those in which the vast majority $(>80 \%)$ of clinical studies included addressed management of pkOA with different modalities than stem cells (three meta-analyses) (Class 3 in Table S5).

Afterwards, the quality of each sub-analysis performed in the remaining 12 meta-analyses (Class 1 in Table S5) was assessed by $\mathrm{CS}$ and $\mathrm{CA}$ according to the quality criteria outlined in Table 1.

\subsection{Systematic assessment of clinical studies on treatment of primary knee osteoarthritis with stem cells}

The stratetgy of the second assessment of the identified publications is summarized in Figure 2. In a first step, CS and CA excluded reviews and investigations that did not represent clinical studies on the management of pkOA with stem cells. Then, each study identified by this search was classified with regard to the type of study as summarized in Tables S2 and S3 and with regard to the type of stem cells used as summarized in Table S4. Furthermore, it was determined which analyses could be performed that fulfilled all quality criteria summarized in Table 1. All this was undertaken by CS and CA. 


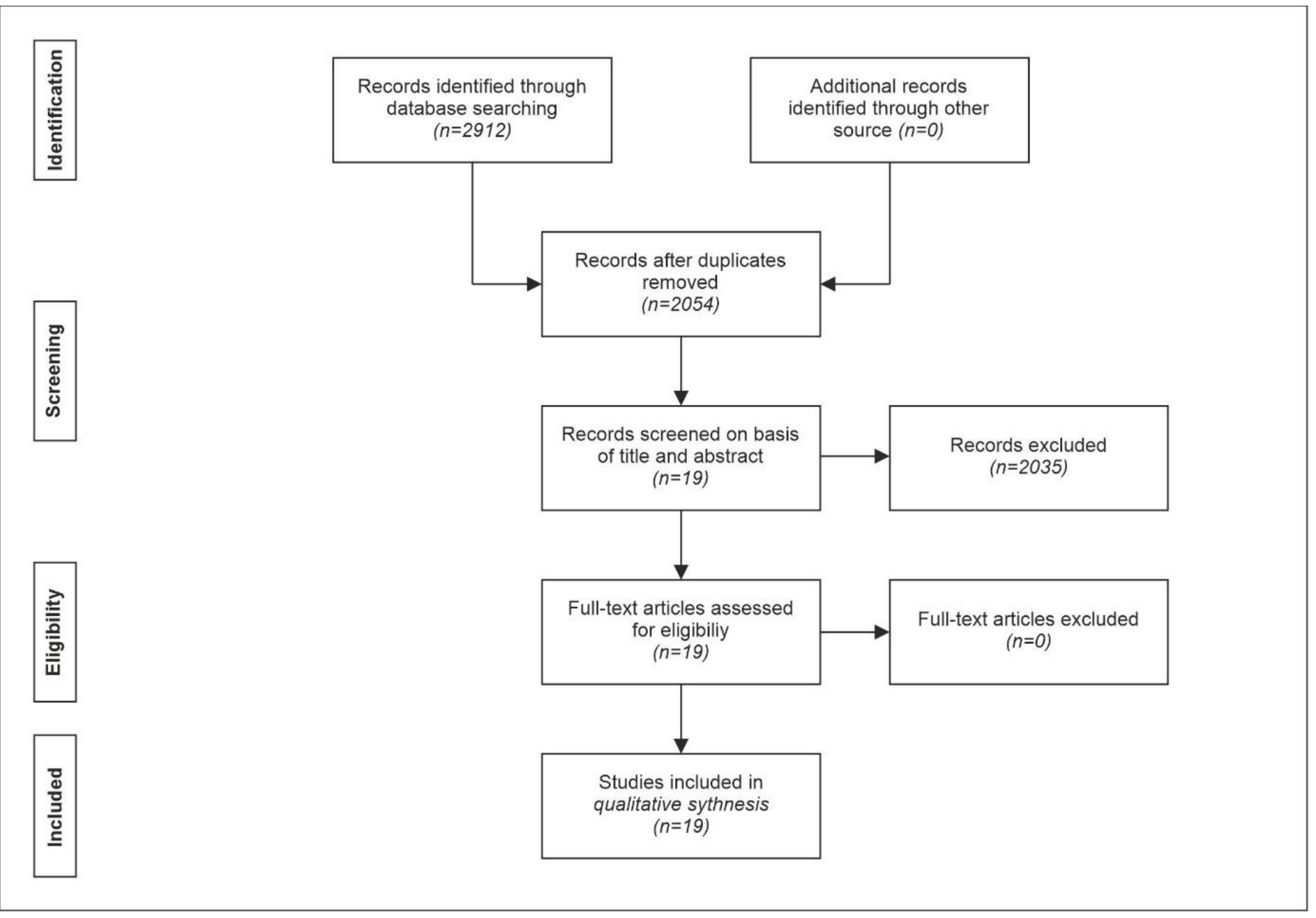

Figure 1 | Systematic review flow chart of the first literature search regarding meta-analyses of clinical studies on treatment of primary knee osteoarthritis with stem cells that were published between January 2020 and July 2021, performed according to the PRISMA guidelines $^{14}$ on 07 August 2021.

\section{Results}

\subsection{Assessment of published meta-analyses}

A total of 56 clinical studies ${ }^{\text {S1-S56 }}$ (note that References S1 to S56 are provided in the Supplement) were included in the 19 assessed meta-analyses; details of these 56 clinical studies are provided in Table S6. Classification of these 56 studies with regard to the type of study is summarized in Tables S2 and S3. Two of these 56 studies $^{\mathrm{S} 55, \mathrm{~S} 56}(3.6 \%)$ could not be evaluated because they are not listed in PubMed, Web of Science and the Cochrane Library, and are not listed in Google Scholar.

Of note, 4 of the 54 studies ${ }^{\mathrm{S} 1, \mathrm{~S} 15, \mathrm{~S} 38, \mathrm{~S} 49}$ that could be evaluated $(7.4 \%)$ did not address pkOA but, respectively, focal chondral, osteochondral, meniscal chondral or meniscal lesions. Furthermore, only 29 of the 54 studies that could be evaluated $(53.7 \%)$ were randomized controlled trials (RCT); the other studies were RCTs with the contralateral knee as internal control $(3 / 54=5.6 \%)$, prospective cohort studies $(7 / 54=13.0 \%)$, retrospective cohort studies $(2 / 54=3.7 \%)$, and case series without control group $(13 / 54=24.1 \%)$ (Table S3).

Thirteen different types of stem cells ( 8 were autologous cell types and 5 were allogeneic cell types) were applied in the
54 studies that could be evaluated, (Table S4). The most frequently applied cell types were autologous, adiposederived stem cells (ADSCs) $(11 / 54=20.4 \%)$, autologous, bone marrow-derived mesenchymal stromal cells (BM-MSCS) $(10 / 54=18.5 \%)$, autologous, adipose derived regenerative cells (ADRCs) $(9 / 54=16.7 \%)$, and autologous bone marrow aspirate concentrate (BMAC) $(7 / 54=13.0 \%)$. In 1 study $^{\mathrm{S} 6}$ no cells but allogeneic amniotic fluid was applied.

Each of the 19 assessed meta-analyses included an average number of $9.3 \pm 4.6$ (mean \pm standard deviation) studies (median, 8; range, 2-18). Conversely, each study listed in Table S6 was included in an average of $3.1 \pm 3.5$ of the 19 assessed meta-analyses (median, 2; range, 1-15). Only 9 of the 56 studies $(16.0 \%)$ were included in 5 or more of the 19 assessed meta-analyses, whereas 26 of the 56 studies $(46.4 \%)$ were included in only 1 of the 19 assessed meta-analyses. None of the 56 studies were included in all assessed metaanalyses; the most frequently included study ${ }^{\mathrm{S} 51}$ was an RCT in which injection of allogeneic BM-MSCs was compared with injection of HA (considered in 15 of the 19 assessed meta-analyses). 
Table 1 | Criteria used to assess the quality of sub-analyses in the meta-analyses summarized in Table S1 of studies in which treatment of primary knee osteoarthritis (pkOA) with different types of stem cells was investigated.

\begin{tabular}{|c|c|c|c|}
\hline No. & Quality criterion & $\mathbf{N}$ & $\mathbf{F}[\%]$ \\
\hline 1 & At least 2 different clinical studies were included. & 141 & 89.8 \\
\hline 2 & Only clinical studies on pkOA were included. & 141 & 89.9 \\
\hline 3 & Only clinical studies in which stem cells were applied were included. & 155 & 98.7 \\
\hline 4 & Only randomized controlled trials were included. & 133 & 84.7 \\
\hline 5 & $\begin{array}{l}\text { Only clinical studies were included in which application of stem cells was compared with placebo } \\
\text { treatment, or in which application of stem cells plus concomitant therapy (including arthroscopic } \\
\text { debridement, high tibial osteotomy, injection of hyaluronic acid, etc.) was compared with the concomitant } \\
\text { therapy alone, respectively. }\end{array}$ & 55 & 35.0 \\
\hline 6 & Only clinical studies using respectively autologous or allogeneic stem cells were included. & 64 & 40.8 \\
\hline 7 & Only clinical studies in which respectively cultured or uncultured cells were applied were included. & 133 & 84.7 \\
\hline 8 & $\begin{array}{l}\text { Clinical studies in which more than } 1 \text { dose of stem cells were applied were only considered once in the } \\
\text { corresponding meta-analysis. }\end{array}$ & 100 & 63.4 \\
\hline
\end{tabular}

Abbreviations: $\mathrm{N}_{\mathrm{A}}$ and $\mathrm{N}_{\mathrm{R}}=$ absolute $\left(\mathrm{N}_{\mathrm{A}}\right)$ and relative $\left(\mathrm{N}_{\mathrm{R}}\right)$ number of sub-analyses that fulfilled the corresponding criterion; No. $=$ number.

In 4 of the 19 assessed meta-analyses ${ }^{19,21,22,31}$, subjective (self-reported pain and function scores) and objective (cartilage measurements) endpoints of the same patients before and after treatment were analyzed. Since this approach cannot rule out the possibility that all reported and measured effects were based on the placebo effect in the management of $\mathrm{pkOA}^{34-36}$, these meta-analyses were excluded from further assessment.

In the 3 network meta-analyses, respectively only two ${ }^{26}$, three ${ }^{23}$ and $\operatorname{six}^{33}$ studies on the management of pkOA with stem cells were included, but respectively $41^{26}, 22^{23}$ and $37^{33}$ studies addressing the management of pkOA without injection of stem cells were included. Since this approach does not at all reflect the actually available literature on the management of pkOA with stem cells (c.f. Table S6), these network metaanalyses were also excluded from further assessment.

In the remaining 12 meta-analyses $15-18,20,24,25,27-30,32$, a total of 157 sub-analyses were performed; details of these 157 subanalyses and individual assessment according to the quality criteria listed in Table 1 are provided in Tables S7-S18. The absolute and relative numbers of sub-analyses that fulfilled respectively $0 / 1 / 2 / 3 / 4 / 5 / 6 / 7 / 8$ of the quality criteria outlined in Table 1 were $0 / 0 / 2 / 3 / 9$ / 18 / 94 / 30 / 1 (or $0 \% / 0 \% / 1.3 \% / 1.9 \% / 5.7 \% / 11.5 \% / 59.9 \% / 19.1 \% / 0.6 \%$, respectively).
The 2 sub-analyses which fulfilled only 2 of the 8 quality criteria outlined in Table 1 are listed in Table S10, and compared the following studies with respect to the VAS pain score at 3 months and 6 months post treatment: a study in which the management of pkOA was performed using allogeneic amniotic fluid ${ }^{\mathrm{S} 6}$, a RCT in which application of allogeneic ADSCs was compared with placebo treatment ${ }^{\mathrm{S} 26}$ (study considered 2 times because of 2 different doses of stem cells), a RCT in which application of autologous BM-MSCs plus HA injection was compared to HA injection alone S27 $^{\text {Hen }}$ (study also considered 2 times because of 2 different doses of stem cells), and a study in which application of autologous BMC plus platelet poor plasma injection was compared with placebo treatment, with the contralateral knee as internal control $^{\mathrm{S} 41}$.

The only sub-analysis fulfilling all quality criteria outlined in Table 1 is listed in Table S15, and compared the following studies with respect to categorical data (worse/not worse) of different MRI evaluations, which were categorical structures with different scales and, thus, converted to categorical data by the authors of this meta-analysis ${ }^{17}$ : a RCT in which the management of pkOA with allogeneic placental mesenchymal stem cells was compared with placebo treatment ${ }^{\mathrm{S} 21}$, and a $\mathrm{RCT}$ in which the management of pkOA with allogeneic ADSCs was also compared with placebo treatment ${ }^{\mathrm{S} 26}$. 


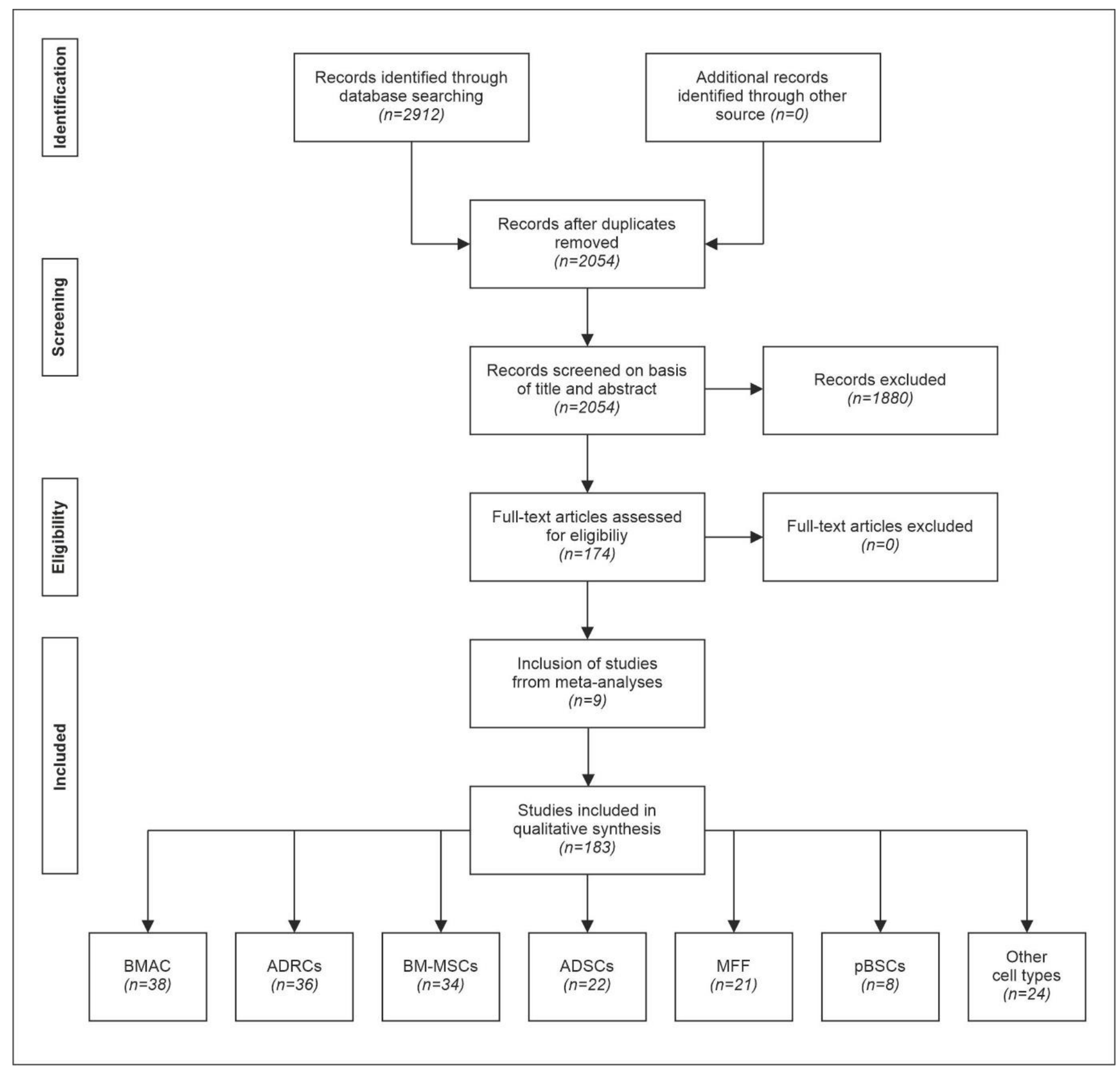

Figure 2 Systematic review flow chart of the second literature search regarding treatment of primary knee osteoarthritis with stem cells, performed according to the PRISMA guidelines ${ }^{14}$ on 07 August 2021. ADRCs $=$ adipose-derived regenerative cells; ADSCs $=$ adipose derived stem cells; BMC = bone marrow concentrate; BM-MSCs = bone marrow-derived mesenchymal stem cells; hUCBMSCs = human umbilical cord blood-derived MSCs; MFF = micro-fragmented fat (from liposuction).

\subsection{Systematic assessment of clinical studies on treatment of} primary knee osteoarthritis with stem cells

Our independent literature search yielded 2912 publications, of which 174 described clinical studies with a total number of $\mathrm{n}=7146$ patients treated with different types of stem cells (treatment groups). The other 2738 publications were duplicates $(n=858)$ or addressed topics other than the management of pkOA with stem cells $(n=681)$, reviews of the literature $(\mathrm{n}=476)$, animal studies $(\mathrm{n}=329)$, in vitro studies $(n=285)$, commentaries $(n=35)$, combined animal and in vitro studies $(n=26)$, conference abstracts $(n=17)$, study protocols $(n=11)$, animal studies on different topics $(n=6)$, reviews on different topics $(n=5)$, position statements $(n=4)$, errata $(n=3)$ and retraction notes $(n=2)$. Eight of the studies listed in Table $\mathrm{S} 1^{\mathrm{S} 3, \mathrm{~S} 7, \mathrm{~S} 12, \mathrm{~S} 22, \mathrm{~S} 38, \mathrm{~S} 46, \mathrm{~S} 50, \mathrm{~S} 56}$ were not found in this literature search, as well as 1 study ${ }^{\mathrm{S} 173}$ that was mentioned but not used in the sub-analyses performed in 1 of the assessed metaanalyses $^{28}$. In these 9 additional studies, a total number of 
$\mathrm{n}=532$ patients were treated with different types of stem cells (treatment groups). Accordingly, the combined literature search (all assessed meta-analyses and the literature search outlined in Figure 2) yielded a total of 183 studies, with a total number of $n=7678$ patients. Details of these 183 studies are provided in Table S19. Categorization of these studies with respect to treatment and control treatment is provided in Table S20, with respect to the type of study in Table S21 and with respect to the types of stem cells used in Table S22. Results of the corresponding combined three-step categorization are summarized in Table 2.

Most of the 183 studies listed in Table S19 were performed using autologous BMAC (38 studies comprising a total of 2905 patients, among them 2588 patients with pkOA), followed by autologous ADRCs (36 studies; 1608 patients; 1568 with pkOA), autologous BM-MSCs (34 studies; 468 patients; 385 patients with pkOA), autologous ADSCs (22 studies; 368 patients; 346 patients with pkOA), microfragmented fat from liposuction (MFF) (21 studies; 1489 patients with pkOA), peripheral blood-derived MSCs ( 8 studies; 183 patients with pkOA; 54 patients with pkOA) and other cell types (24 studies; 657 patients; 430 patients with pkOA). Of note, in none of these 183 studies a serious adverse event after application of stem cells was reported.

Management of pkOA with stem cells was performed on a total of $n=6860$ patients in 143 of the 183 studies (Categories $\mathrm{I}-\mathrm{V}$ in Table 2 and Table S20). The remaining 40 clinical studies (including a total of 818 patients) addressed respectively focal chondral, osteochondral, meniscal chondral or meniscal lesions (Category VI in Table 2 and Table S20), pathologies which are not addressed in the present setting.

In 65 of these 143 clinical studies on the management of pkOA, treatment with stem cells was compared to sham treatment or another treatment (RCTs or prospective or retrospective two- or three-cohort studies, respectively) (Categories I-IV in Table 2 and Table S20). On the other hand, the management of pkOA with i.a. injection of stem cells as the sole treatment (not considering rehabilitation) was performed in only 38 of these 65 studies (Categories I-III in Table 2 and Table S20), and i.a. injection of respectively saline or sham treatment was only performed in 8 of these 38 studies (Category I in Table 2 and Table S20).

According to the quality criteria of meta-analyses of studies on the management of pkOA with stem cells outlined in Table 1 , it would generally be possible to perform the sub-analyses shown in Table 3. However, MFF must not be confused with $\mathrm{ADRCs}^{37,38}$, and there are substantial differences between cultured stem cells derived from various human tissues ${ }^{39}$. The latter argument also applies to the 56 studies included in the 19 assessed meta-analyses, which further diminishes the value of these meta-analyses as assessment of the management of pkOA with stem cells.

Furthermore, a meta-analysis requires that in all included studies the same type of data is reported (i.e., same assessment scores, same or very similar intervals between treatment and follow-up, and mean, standard deviation and number of patients in each group). As shown in Table 4, this was not achieved for the clinical studies on the management of pkOA with respectively autologous ADRCs, autologous ADSCs and autologous BM-MSCs listed in Table 3.

\section{Discussion}

Concerning the first quality criterion outlined in Table 1, it should be mentioned that a meta-analysis is a statistical analysis that combines the results of multiple scientific studies. Hence, calculating overall effects based on a single study should not be considered a meta-analysis. However, calculating overall effects based on a single study is exactly what was done in 5 of the 19 assessed meta-analyses $15,20,24,25,29$ and 16 of the $157(10.2 \%)$ sub-analyses listed in Tables S7S18.

With respect to the second and third quality criteria outlined in Table 1, it appears obvious that, in meta-analyses of studies in which the management of pkOA with stem cells was investigated, only studies focusing on the management of pkOA and applying stem cells should be included. However, this was not always the case in the 19 assessed meta-analyses. The pathomechanisms of focal chondral, osteochondral or meniscal chondral lesions ${ }^{\mathrm{S} 1, \mathrm{~S} 15, \mathrm{~S} 38}$ as well as of injuries that require partial medial meniscectomy ${ }^{\mathrm{S} 49}$ are not the same as the pathomechanisms of pkOA. This will likely impact on treatment outcome when applying stem cells. Furthermore, despite the fact that stem cells can be isolated from amniotic fluid $^{40}$, in our opinion injection of allogeneic amniotic fluid itself $^{\mathrm{S} 6}$ should not be considered a stem cell treatment, as it does not contain, by definition, stems cells, though it may contain products of secretion by stem cells.

Concerning the fourth quality criterion outlined in Table 1, non-randomized clinical trials and studies in which the contralateral knee was used as internal control may differ from RCTs in terms of selection bias (result of systematic differences between baseline characteristics of the groups that are compared ${ }^{41}$ ), performance bias (result of systematic differences between groups in the care that is provided, or in exposure to factors other than the interventions of interest ${ }^{42}$ ), and attrition bias (result of systematic differences between groups in withdrawals from a study ${ }^{41}$ ). Accordingly, nonrandomized clinical trials and studies in which the contralateral knee was used as internal control should not be combined with RCTs in meta-analyses. However, this quality criterion was not fulfilled in 24 of the 157 (15.3\%) subanalyses performed in the 12 assessed meta-analyses (Category 1 in Table S5). 
Table 2 Detailed analysis of clinical studies on the management of pkOA with stem cells (summarized in Table S19) that were identified during an evidence-based, systematic review of the literature according to the PRISMA guidelines ${ }^{14}$ performed on August 07, 2021. The categories of studies (I to VI) are explained in Table S20, and the types of study (a to d) in Table S21.

\begin{tabular}{|c|c|c|c|c|c|c|c|c|c|c|c|c|c|c|c|c|c|c|c|c|c|c|c|c|c|c|}
\hline \multirow{2}{*}{$\begin{array}{l}\text { Category } \\
\text { Type of study }\end{array}$} & \multicolumn{4}{|c|}{$\mathbf{I}$} & \multicolumn{4}{|c|}{ II } & \multicolumn{4}{|c|}{ III } & \multicolumn{4}{|c|}{ IV } & \multicolumn{2}{|c|}{$\mathbf{V}$} & \multicolumn{6}{|c|}{$V I$} & \multirow[t]{2}{*}{$\mathbf{N}_{\text {pkOA }}$} & \multirow[t]{2}{*}{$N_{C L}$} \\
\hline & $\mathbf{a}$ & b & c & d & $\mathbf{a}$ & b & c & d & $\mathbf{a}$ & b & c & d & $\mathbf{a}$ & b & c & d & e & f & $a$ & $b$ & $c$ & $d$ & $\boldsymbol{e}$ & $f$ & & \\
\hline ADRCs & 1 & & & & & & & 1 & & & 1 & 2 & 2 & 1 & 5 & 1 & 20 & 1 & 1 & & & & & & 1568 & 40 \\
\hline ADSCs & 2 & & & & 1 & & & & 4 & & 5 & & 1 & & 1 & & 3 & 1 & 1 & & & & 1 & 2 & 346 & 22 \\
\hline MFF & & & & & 1 & & & & & & 2 & 1 & 1 & & & 1 & 15 & & & & & & & & 1489 & \\
\hline CLL & & & & & & & & & & & & & & & & & 1 & & & & & & & & 20 & \\
\hline $\mathrm{BMA}$ & & & & & & & & & & & & & & & & & 1 & & & & & & & & 3 & \\
\hline BMAC & & 1 & & & 2 & & & & 1 & 3 & & & 1 & 1 & & 4 & 13 & & 1 & & 3 & 1 & 6 & 1 & 2588 & 317 \\
\hline BM-MSCs & 1 & & & & 1 & & & & 1 & & 1 & 1 & 7 & & & & 11 & 2 & 3 & & & & 4 & 2 & 385 & 83 \\
\hline $\mathrm{Cs} / \mathrm{CPs}$ & & & & & & & & & & & & & & & & & & & & & & & 1 & 1 & & 13 \\
\hline $\mathrm{CSCs}$ & & & & & & & & & & & & & & & & & & & & & & & 1 & & & 15 \\
\hline MACI & & & & & & & & & & & & & & & & & & & & & & & 1 & & & 15 \\
\hline S-MSCs & & & & & & & & & & & & & & & & & 1 & & 1 & & & & 2 & & 8 & 18 \\
\hline Ch-TGFß & 2 & & & & & & & & & & & & & & & & & & & & & & & & 128 & \\
\hline hUC-MSCs & & & & & 2 & & & & & & & & & & & & 1 & & & & & & 1 & & 75 & $*$ \\
\hline hUCB-MSCs & & & & & & & & & & & & & & & & & 5 & & 1 & & & & 1 & & 186 & 166 \\
\hline P-MSCs & 1 & & & & & & & & & & & & & & & & & & & & & & & & 10 & \\
\hline pBSCs & & & & & & & & & & & & & 1 & & & & 3 & & 3 & & & & 1 & & 54 & 129 \\
\hline Sum & 7 & 1 & $\mathbf{0}$ & $\mathbf{0}$ & 7 & $\mathbf{0}$ & $\mathbf{0}$ & 1 & 6 & 3 & 9 & 4 & 13 & 2 & 6 & 6 & 74 & 4 & 11 & 0 & 3 & 1 & 19 & 6 & 6860 & 818 \\
\hline
\end{tabular}

Abbreviations: $\mathrm{ADRCs}=$ adipose-derived regenerative cells; $\mathrm{ADSCs}=$ adipose-derived stem cells (obtained by culturing ADRCs); $\mathrm{BMA}=$ bone marrow aspirate; $\mathrm{BMAC}=$ bone marrow concentrate; BM-MSCs = bone marrow-derived mesenchymal stromal cells; CLL = centrifuged liposuction liquid; Cs = chondrocytes; $\mathrm{CPs}=$ chondrocyte precursors; $\mathrm{CSCs}$ $=$ cartilage stem cells; hUC-MSCs = human umbilical cord-derived MSCs; hUCB-MSCs = human umbilical cord blood-derived MSCs; MACI = matrix-induced autologous chondrocyte implant; $\mathrm{MFF}=$ micro-fragmented fat (from liposuction); $\mathrm{N}_{\mathrm{CL}}=$ number of patients with chondral lesions treated in these studies; $\mathrm{N}_{\mathrm{pkOA}}=$ number of patients with pKOA treated in these studies; pBSCs $=$ activated peripheral blood stem cells; P-MSCs $=$ placental MSCs; S-MSCs $=$ matrix-induced MSCs from synovia; $*=$ number of patients not provided. 
Table 3 | Sub-analyses that would generally be possible in a meta-analysis of studies on treatment of primary knee osteoarthritis with stem cells (summarized in Table S19) that were identified during an evidence-based, systematic review of the literature according to the PRISMA guidelines ${ }^{14}$ performed on August 07, 2021.

\begin{tabular}{|c|c|c|c|c|c|c|c|}
\hline $\mathbf{R}$ & $\mathbf{C a}$ & $\mathbf{T}$ & First author & $\mathbf{Y}$ & Cell type & Treatment & Control \\
\hline \multicolumn{8}{|c|}{ Autologous, uncultured cells } \\
\hline S11 & 1 & $\mathrm{a}$ & Garza & 2020 & ADRCs & $\mathrm{C}$ & RS \\
\hline S25 & 4 & a & Koh & 2014 & ADRCs & $\mathrm{C}+\mathrm{AD}+\mathrm{HTO}+\mathrm{PRP}$ & $\mathrm{AD}+\mathrm{HTO}+\mathrm{PRP}$ \\
\hline S149 & 4 & a & Peretti & 2018 & MFF & $\mathrm{C}+\mathrm{AD}$ & $\mathrm{AD}$ \\
\hline \multicolumn{8}{|c|}{ Autologous, cultured cells } \\
\hline S28 & 1 & $\mathrm{a}$ & Lee & 2019 & ADSCs & $\mathrm{C}$ & $\mathrm{Sa}$ \\
\hline S9 & 3 & a & Freitag & 2019 & ADSCs & $\mathrm{C}$ & $\mathrm{CM}$ \\
\hline S56 & 4 & a & Zhang & 2018 & ADSCs & $\mathrm{C}+\mathrm{HA}$ & $\mathrm{C}$ \\
\hline S152 & 4 & a & Qiao & 2020 & ADSCs & $\mathrm{C}+\mathrm{AD}+\mathrm{MF}+\mathrm{HA}$ & MF or MF + HA \\
\hline S8 & 1 & $\mathrm{a}$ & Emadedin & 2018 & BM-MSCs & $\mathrm{C}$ & $\mathrm{Sa}$ \\
\hline S50 & 4 & a & Varma & 2010 & BM-MSCs & $\mathrm{C}+\mathrm{AD}$ & $\mathrm{AD}$ \\
\hline S53 & 4 & a & Wong & 2013 & BM-MSCs & $\mathrm{C}+\mathrm{MF}+\mathrm{HTO}+\mathrm{HA}$ & $\mathrm{MF}+\mathrm{HA}+\mathrm{HTO}$ \\
\hline S27 & 4 & a & Lamo-Espinosa & 2016 & BM-MSCs & $\mathrm{C}+\mathrm{HA}$ & HA \\
\hline S5 & 4 & a & Bastos & 2018 & BM-MSCs & $\mathrm{C}+\mathrm{PRP}$ & $\mathrm{C}$ \\
\hline S128 & 4 & a & Lamo-Espinosa & 2018 & BM-MSCs & $\mathrm{C}+\mathrm{HA}$ & HA \\
\hline S127 & 4 & $\mathrm{a}$ & Lamo-Espinosa & 2020 & BM-MSCs & $\mathrm{C}+\mathrm{PRP}$ & PRP \\
\hline \multicolumn{8}{|c|}{ Allogeneic, cultured cells } \\
\hline S7 & 1 & $\mathrm{a}$ & Cherian & 2015 & Ch-TGFß & $\mathrm{C}$ & $\mathrm{Sa}$ \\
\hline S13 & 4 & a & Gupta & 2016 & BM-MSCs & $\mathrm{C}+\mathrm{HA}$ & $\mathrm{S}+\mathrm{HA}$ \\
\hline S22 & 1 & a & Kim & 2018 & Ch-TGFß & $\mathrm{C}$ & $\mathrm{Sa}$ \\
\hline S26 & 1 & a & Kuah & 2018 & ADSCs & $\mathrm{C}$ & $\mathrm{Sa}$ \\
\hline S21 & 1 & $\mathrm{a}$ & Khalifeh Soltani & 2019 & P-MSCs & $\mathrm{C}$ & $\mathrm{Sa}$ \\
\hline
\end{tabular}

Abbreviations: $\mathrm{AD}=$ arthroscopic debridement; $\mathrm{ADRCs}=$ adipose-derived regenerative cells; $\mathrm{ADSCs}=$ adipose-derived stem cells; BM-MSCs = bone marrow-derived mesenchymal stromal cells; $\mathrm{C}=$ cells; $\mathrm{Ca}=$ category of study as outlined in Table S20; Ch-TGFß = chondrocytes that overexpress transcription growth factor beta; HTO = high tibial osteotomy; MF = microfracture; MFF = microfragmented fat; P-MSCs = placental MSCs; PRP = platelet rich plasma; RS = Ringer solution; $\mathrm{S}=$ reference number (note that these references are provided in the Supplemental Online Material); $\mathrm{Sa}=$ saline; $\mathrm{T}=$ type of study as outlined in Table $\mathrm{S} 21$; $\mathrm{Y}=\mathrm{year}$ of publication.

With respect to the fifth quality criterion outlined in Table 1 , it is crucial to bear in mind that studies in which the management of pkOA with stem cells was compared to placebo treatment (or studies in which the management of pkOA with stem cells plus a concomitant therapy was compared to the concomitant therapy alone, respectively) must not be combined with studies in which the management of pkOA with stem cells (with or without concomitant therapy) was compared to a different treatment. However, this quality criterion was not fulfilled in 102 of the $157(65.0 \%)$ sub-analyses performed in the 12 assessed meta-analyses. Specifically, in 86 of the 157 (54.8\%) sub-analyses performed in the 12 assessed meta-analyses studies in which the management of pkOA with stem cells was compared with placebo treatment (or studies in which the management of pkOA with stem cells plus concomitant therapy was compared with the concomitant therapy alone, respectively) were combined with studies in which the management of pkOA with stem cells was compared with injection of HA. However, this approach disregards documented, positive effects of injection of $\mathrm{HA}$ as the management of $\mathrm{pkOA}^{9-11}$, and may thus substantially underestimate the positive effects of treating pkOA with stem cells in meta-analyses. Furthermore, in 9 of the $157(5.7 \%)$ sub-analyses performed in the 12 assessed meta-analyses, only studies in which the management of pkOA with stem cells was compared with the management of pkOA with injection of HA were included. Because of the documented, positive effects of injection of HA injection as the management of $\mathrm{pkOA}^{9-11}$, these sub-analyses may reach a different conclusion than sub-analyses in which only studies were included in which the management of pkOA with stem cells was compared with placebo treatment (or studies in which the management of pkOA with stem cells plus concomitant therapy was compared with the concomitant therapy alone, respectively). 
Table 4 Reported outcome in studies on treatment of primary knee osteoarthritis with respectively autologous, adipose-derived regenerative cells (ADRCs) or autologous, adipose-derived stem cells (ADSCs) listed in Table 3.

\begin{tabular}{|c|c|c|}
\hline $\begin{array}{l}\text { Table } 4 \\
\text { (cont.) R }\end{array}$ & FA & Reported outcome \\
\hline \multicolumn{3}{|l|}{ ADRCs } \\
\hline S11 & Garza & $\begin{array}{l}\text { - WOMAC Total score at BL and at } \mathrm{W} 6, \mathrm{M} 3, \mathrm{M} 6 \text { and M12 (mean, median, interquartile range, } \\
\text { median percentage range, minimum, maximum) } \\
\text { - Cartilage loss (mean at BL, mean change at M6) } \\
\text { - Outerbridge classification (median at BL, range at BL, median change at M6) }\end{array}$ \\
\hline S25 & Koh & $\begin{array}{l}\text { - VAS pain score (mean and SD at baseline and at last follow-up) } \\
\text { - KOOS subscores (mean improvement from BL to LFU) } \\
\text { - Lysholm score (mean and SD at BL and LFU) } \\
\text { - Weight-bearing line }(\%) \text { (mean and SD at BL and LFU) } \\
\text { - Femorotibial angle }\left(^{\circ}\right) \text { (mean and SD at BL and LFU) }\end{array}$ \\
\hline \multicolumn{3}{|c|}{ Autologous ADRCs } \\
\hline $\mathrm{S} 28$ & Lee & $\begin{array}{l}\text { - VAS Pain score at BL, M3 and M6 (mean) } \\
\text { - WOMAC Total and subscores at BL, M3 and M6 (mean) } \\
\text { - KOOS subscores at BL, M3 and M6 (mean) } \\
\text { - Size of cartilage defect in MRI at BL and M6 (mean, SD) }\end{array}$ \\
\hline S9 & Freitag & $\begin{array}{l}\text { - VAS Pain score at BL, M1,5, M3, M6 and M12 (mean, 95\% CI) } \\
\text { - KOOS subscores at BL, M1,5, M3, M6 and M12 (mean, 95\% CI) } \\
\text { - WOMAC score at BL, M1,5, M3, M6 and M12 (mean, 95\% CI) }\end{array}$ \\
\hline S56 & Zhang & $\begin{array}{l}\text { - VAS Pain score at BL, M3, M6, M12, M24 and M36 (mean, SD) } \\
\text { - WOMAC Total score at BL, M3, M6, M12, M24 and M36 (mean, SD) }\end{array}$ \\
\hline S152 & Qiao & $\begin{array}{l}\text { - WOMAC Total and subscores at BL, M3, M6, M9, M12 and M24 (mean, SD) } \\
\text { - SF-36 Physical Component and Mental Component subscores at BL, M3, M6, M9, M12 and M24 } \\
\text { (mean, SD) }\end{array}$ \\
\hline \multicolumn{3}{|c|}{ Autologous ADRCs } \\
\hline $\mathrm{S} 8$ & Emadedin & $\begin{array}{l}\text { - Walking distance, painless walking distance, VAS Pain score, standing time, WOMAC total and } \\
\text { subscores, time to gelling, flexion of knee, MCII Pain and Function scores, PASS Pain and } \\
\text { Function scores (changes at M3 and M6 compared to BL; mean, range, standard error of difference, } \\
\text { 95\% CI of difference) }\end{array}$ \\
\hline S50 & Varma & $\begin{array}{l}\text { - No access; this publication is not listed on the homepage of the Journal of the Indian Medical } \\
\text { Association (https://www.ima-india.org/ima/left-side-bar.php?pid=347) }\end{array}$ \\
\hline S53 & Wong & $\begin{array}{l}\text { - IKDC score, Lysholm score and Tegner score at BL and M6, M12 and M24 (mean, plotted } \\
\text { individual data) }\end{array}$ \\
\hline S27 & Lamo-Espinosa & $\begin{array}{l}\text { - VAS Pain score, WOMAC Total and subscores, range of motion, WORMS score at BL, M3, M6 } \\
\text { and M12 (median, interquartile range) }\end{array}$ \\
\hline $\mathrm{S} 128$ & Lamo-Espinosa & $\begin{array}{l}\text { - VAS Pain score, WOMAC Total score at BL, M3, M6, M12 and post-trial follow-up (median, } \\
\text { interquartile range) }\end{array}$ \\
\hline S127 & Lamo-Espinosa & - VAS Pain score, WOMAC Total and subscores at 3, 6 and 12 months (mean, SD) \\
\hline
\end{tabular}

Abbreviations: $\mathrm{BL}=$ baseline; $\mathrm{CI}=$ confidence interval; $\mathrm{FA}=$ first author; LFU = last follow-up (range, M14-M24; mean: M19.8); M3 / M6 / M9 / M12 / M24 = 3 / 6 / 9 / 12 / 24 months post treatment; R = reference number (note that these references are provided in the Online Supplementary Material); SD = standard deviation; W6 = 6 weeks post treatment.

Concerning the sixth quality criterion outlined in Table 1, it is of note that, in contrast to autologous cells, application of allogeneic cells bears the risk of HLA mismatch; compromised clinical outcome after application of allogeneic cells was repeatedly reported in the literature. ${ }^{43-45} \mathrm{In}$ a position statement recently published by representatives of the U.S. Food and Drug Administration (FDA) in The New England Journal of Medicine $e^{46}$, it was stated that autologous stem cells may typically raise fewer safety concerns than allogeneic stem cells. Consequently, studies using autologous stem cells should not be combined with studies using allogeneic stem cells in meta-analyses. However, this quality criterion was not fulfilled in 93 of the 157 (59.2\%) sub-analyses performed in the 12 assessed meta-analyses.

With respect to the seventh quality criterion outlined in Table 1, ADSCs and BM-MSCs are expanded in culture before application, whereas ADRCs and BMAC are not. As a consequence, the percentage of certain cell types in ADSCs 
fundamentally differs from the percentage of the same cell types in $\mathrm{ADRCs}{ }^{38}$, and the percentage of certain cell types in BM-MSCs fundamentally differs from the percentage of the same cell types in $\mathrm{BMAC}^{47}$. Hence, in meta-analyses of studies in which pkOA was treated with stem cells studies in which cultured cells were applied should not be combined with studies in which uncultured cells were applied. However, this quality criterion was not fulfilled in 24 of the 157 (15.3\%) sub-analyses performed in the 12 assessed meta-analyses.

Concerning the eighth quality criterion outlined in Table 1, it should be realized that each study has its own inclusion and exclusion criteria which may vary among studies. Furthermore, there are many different ways to prepare a certain type of stem cells (e.g., approximately 40 different approaches to isolate ADRCs from adipose tissue are reported in the literature, with substantial inter-individual variations in the final cell suspensions ${ }^{37}$ ). Accordingly, considering the same study more than once in a meta-analysis can introduce bias (such as considering the 4 groups of patients treated $i^{\mathrm{S} 13}$ with 4 different doses of allogeneic BM-MSCs as 4 studies in a certain meta-analysis). However, this quality criterion was not fulfilled in 57 of the 157 (36.3\%) sub-analyses performed in the 12 assessed meta-analyses.

We cannot offer any explanation why the 19 assessed metaanalyses did not adhere to the quality criteria summarized in Table 1. As outlined in detail above, these quality criteria reflect general considerations about meta-analyses and the very basic biology of stem cells.

\subsection{Limitations}

This review had two limitations. First, only PubMed, Web of Science and the Cochrane Library were searched. However, considering the sophisticated search strategies described in the 19 assessed meta-analyses and inclusion of all studies that were included in the 19 assessed meta-analyses also in the present investigation minimized the risk to overlook any relevant clinical study on the management of pkOA with stem cells. Second, no meta-analyses published before 2020 were assessed. However, this does not devalue our finding that the 19 meta-analyses published from January 2020 to July 2021 were not scientifically sound.

\section{Conclusions}

The inconsistent conclusions of the 19 assessed meta-analyses regarding the efficacy and safety of treating pkOA with stem cells were most probably based on substantial inter-individual differences in literature search strategies among different authors, misconceptions about meta-analyses themselves and misconceptions about the biology of stem cells. None of the 19 assessed meta-analyses should be considered to provide a scientifically definitive assessment of the efficacy of treating pkOA with stem cells. Accordingly, clinicians should be cautious of the 19 assessed meta-analyses of clinical studies on the management of pkOA with stem cells and the conclusions drawn therein, and strive to participate in FDA and/or EMA approved trials that have been reviewed to provide clinically and statistically valid efficacy.

Furthermore, at this time it appears impossible to perform a scientifically sound meta-analysis of such a heterogenous set of studies which investigated the management of pkOA with stem cells. There are strong indications that the management of pkOA with stem cells is safe and effective (e.g., all RCTs in Category I in Table S20 reported superiority of the management of pkOA over treatment with placebo). However, apparently it is still too early for scientifically sound metaanalyses on this topic.

\section{Acknowledgments}

This study did not receive external funding.

\section{Authors' contributions}

CS participated in conceptualization, development of methodology, formal analysis and investigation; managed resources, and performed data curation, writing of the original draft, visualization and project administration. CA participated in validation, formal analysis and investigation, as well as in reviewing and editing of the manuscript. DAP participated in validation as well as reviewing and editing of the manuscript. JPF and NM participated in reviewing and editing of the manuscript. EUA participated in conceptualization as well as reviewing and editing of the manuscript, and in project administration and supervision. All authors have read and approved the final version of the manuscript, and agree with the order of presentation of the authors.

\section{Competing interests}

C.S. is Advisory Medical Director of InGeneron, Inc. (Houston, TX). C.A. is Director of Medical and Scientific Affairs of InGeneron. E.U.A. is Executive Chair of InGeneron. InGeneron had no role in study design, data collection and analysis, interpretation of the data, and no role in the decision to publish and write this manuscript. No other potential conflicts of interest relevant to this article were reported.

\section{Supplementary materials}

Supplementary material associated with this article can be found at the end of this preprint.

\section{REFERENCES}

1. Felson DT. Clinical practice. Osteoarthritis of the knee. $N$ Engl $J$ Med 2006;354:841-848. doi: 10.1056/NEJMcp051726.

2. World report on disability. Geneva: World Health Organization, 2011

https://www.who.int/disabilities/world_report/2011/report.pdf?ua= 1. Accessed November 12, 2021.

3. Loeser RF, Goldring SR, Scanzello CR, et al. Osteoarthritis: a disease of the joint as an organ. Arthritis Rheum 2012;64:1697-1707. doi: 10.1002/art.34453.

4. Fransen M, Agaliotis M, Bridgett L, et al. Hip and knee pain: role of occupational factors. Best Pract Res Clin Rheumatol 2011;25:81101. doi: 10.1016/j.berh.2011.01.012.

5. Salaffi F, Ciapetti A, Carotti M. The sources of pain in osteoarthritis: a pathophysiological review. Reumatismo 2014;66:57-71. doi: 10.4081/reumatismo.2014.766.

6. Lluch Girbés E, Nijs J, Torres-Cueco R, et al. Pain treatment for 
patients with osteoarthritis and central sensitization. Phys Ther 2013;93:842-851. doi: 10.2522/ptj.20120253.

7. Carr AJ, Robertsson O, Graves S, et al. Knee replacement. Lancet 2012;379:1331-40. doi: 10.1016/S0140-6736(11)60752-6.

8. Skou ST, Roos EM, Laursen MB, et al. A randomized, controlled trial of total knee replacement. N Engl J Med 2015;373:1597-1606. doi: 10.1056/NEJMoa1505467.

9. Jüni P, Hari R, Rutjes AW, et al. Intra-articular corticosteroid for knee osteoarthritis. Cochrane Database Syst Rev 2015;(10):CD005328. doi: 10.1002/14651858.CD005328.pub3.

10. Meheux CJ, McCulloch PC, Lintner DM, et al. Efficacy of intraarticular platelet-rich plasma injections in knee osteoarthritis: a systematic review. Arthroscopy 2016;32:495-505. doi: 10.1016/j.arthro.2015.08.005.

11. Ran J, Yang X, Ren Z, et al. Comparison of intra-articular hyaluronic acid and methylprednisolone for pain management in knee osteoarthritis: A meta-analysis of randomized controlled trials. Int $J$ Surg 2018;53:103-10. doi: 10.1016/j.ijsu.2018.02.065.

12. Bannuru RR, Osani MC, Vaysbrot EE, et al. OARSI guidelines for the non-surgical management of knee, hip, and polyarticular osteoarthritis. Osteoarthritis Cartilage 2019;27:1578-89. doi: 10.1016/j.joca.2019.06.011.

13. He WW, Kuang MJ, Zhao J, et al. Efficacy and safety of intraarticular hyaluronic acid and corticosteroid for knee osteoarthritis: A meta-analysis. Int J Surg 2017;39:95-103. doi: 10.1016/j.ijsu.2017.01.087.

14. Liberati A, Altman DG, Tetzlaff J, et al. The PRISMA statement for reporting systematic reviews and meta-analyses of studies that evaluate healthcare interventions: explanation and elaboration. $B M J$. 2009;339:b2700. doi: 10.1136/bmj.b2700.

15. Han X, Yang B, Zou F, et al. Clinical therapeutic efficacy of mesenchymal stem cells derived from adipose or bone marrow for knee osteoarthritis: a meta-analysis of randomized controlled trials. J Comp Eff Res 2020;9:361-74. doi: 10.2217/cer-2019-0187.

16. Huang R, Li W, Zhao Y, et al. Clinical efficacy and safety of stem cell therapy for knee osteoarthritis: A meta-analysis. Medicine (Baltimore) 2020;99:e19434. doi: 10.1097/MD.0000000000019434.

17. Kim SH, Djaja YP, Park YB, et al. Intra-articular injection of culture-expanded mesenchymal stem cells without adjuvant surgery in knee osteoarthritis: a systematic review and meta-analysis. Am J Sports Med 2020;48:2839-49. doi: 10.1177/0363546519892278.

18. Ma W, Liu C, Wang S, et al. Efficacy and safety of intra-articular injection of mesenchymal stem cells in the treatment of knee osteoarthritis: A systematic review and meta-analysis. Medicine (Baltimore) 2020;99:e23343. doi: 10.1097/MD.0000000000023343.

19. Song Y, Zhang J, Xu H, et al. Mesenchymal stem cells in knee osteoarthritis treatment: A systematic review and meta-analysis. $J$ Orthop Translat 2020;24:121-30. doi: 10.1016/j.jot.2020.03.015.

20. Wang J, Zhou L, Zhang Y, et al. Mesenchymal stem cells - a promising strategy for treating knee osteoarthritis. Bone Joint Res 2020;9:719-28. doi: 10.1302/2046-3758.910.BJR-2020-0031.R3.

21. Agarwal N, Mak C, Bojanic C, et al. Meta-analysis of adipose tissue derived cell-based therapy for the treatment of knee osteoarthritis. Cells 2021;10:1365. doi: 10.3390/cells10061365.

22. Bolia IK, Bougioukli S, Hill WJ, et al. Clinical efficacy of bone marrow aspirate concentrate versus stromal vascular fraction injection in patients with knee osteoarthritis: a systematic review and meta-analysis. Am J Sports Med 2021:3635465211014500. Epub ahead of print. doi: 10.1177/03635465211014500.

23. Cao Z, Li Y, Gao F, et al. Mesenchymal stem cells: a new choice for nonsurgical treatment of OA? results from a Bayesian network metaanalysis. Biomed Res Int 2021;2021:6663003. doi: $10.1155 / 2021 / 6663003$.

24. Dai W, Leng X, Wang J, et al. Intra-articular mesenchymal stromal cell injections are no different from placebo in the treatment of knee osteoarthritis: a systematic review and meta-analysis of randomized controlled trials. Arthroscopy 2021;37:340-58. doi: 10.1016/j.arthro.2020.10.016.
25. Ding W, Xu YQ, Zhang Y, et al. Efficacy and safety of intra-articular cell-based therapy for osteoarthritis: systematic review and network meta-analysis. Cartilage 2021;13:104S-15S. doi: 10.1177/1947603520942947. doi: 10.1177/1947603520942947.

26. Han SB, Seo IW, Shin YS. Intra-articular injections of hyaluronic acid or steroids associated with better outcomes than platelet-rich plasma, adipose mesenchymal stromal cells, or placebo in knee osteoarthritis: a network meta-analysis. Arthroscopy 2021;37:292306. doi: 10.1016/j.arthro.2020.03.041.

27. Jeyaraman M, Muthu S, Ganie PA. Does the source of mesenchymal stem cell have an effect in the management of osteoarthritis of the knee? Meta-analysis of randomized controlled trials. Cartilage 2021;13:1532S-47S. doi: 10.1177/1947603520951623.

28. Jiang P, Mao L, Qiao L, et al. Efficacy and safety of mesenchymal stem cell injections for patients with osteoarthritis: a meta-analysis and review of RCTs. Arch Orthop Trauma Surg 2021;141:1241-51. doi: 10.1007/s00402-020-03703-0.

29. Maheshwer B, Polce EM, Paul K, et al. Regenerative potential of mesenchymal stem cells for the treatment of knee osteoarthritis and chondral defects: a systematic review and meta-analysis. Arthroscopy 2021;37:362-78. doi: 10.1016/j.arthro.2020.05.037.

30. Qu H, Sun S. Efficacy of mesenchymal stromal cells for the treatment of knee osteoarthritis: a meta-analysis of randomized controlled trials. J Orthop Surg Res 2021;16:11. doi: 10.1186/s13018-020-02128-0.

31. Tan SHS, Kwan YT, Neo WJ, et al. Intra-articular injections of mesenchymal stem cells without adjuvant therapies for knee osteoarthritis: a systematic review and meta-analysis. Am J Sports Med 2021;49:3113-24. doi: 10.1177/0363546520981704.

32. Wei ZJ, Wang QQ, Cui ZG, et al. Which is the most effective one in knee osteoarthritis treatment from mesenchymal stem cells obtained from different sources?-A systematic review with conventional and network meta-analyses of randomized controlled trials. Ann Transl Med 2021;9:452. doi: 10.21037/atm-20-5116.

33. Zhao D, Pan JK, Yang WY, et al. Intra-articular injections of platelet-rich plasma, adipose mesenchymal stem cells, and bone marrow mesenchymal stem cells associated with better outcomes than hyaluronic acid and saline in knee osteoarthritis: a systematic review and network meta-analysis. Arthroscopy 2021;37:2298314.e10. doi: 10.1016/j.arthro.2021.02.045.

34. Dieppe P, Goldingay S, Greville-Harris M. The power and value of placebo and nocebo in painful osteoarthritis. Osteoarthritis Cartilage 2016;24:1850-7. doi: 10.1016/j.joca.2016.06.007.

35. Zhang W. The powerful placebo effect in osteoarthritis. Clin Exp Rheumatol 2019;37 Suppl 120:118-23.

36. Previtali D, Merli G, Di Laura Frattura G, et al. The long-lasting effects of "placebo injections" in knee osteoarthritis: a meta-analysis. Cartilage 2021;13:185S-96S. doi: 10.1177/1947603520906597. doi: $10.1177 / 1947603520906597$.

37. Winnier GE, Valenzuela N, Peters-Hall J, et al. Isolation of adipose tissue derived regenerative cells from human subcutaneous tissue with or without the use of an enzymatic reagent. PLoS One 2019;14:e0221457. doi: 10.1371/journal.pone.0221457.

38. Alt EU, Winnier G, Haenel A, et al. Towards a comprehensive understanding of UA-ADRCs (uncultured, autologous, fresh, unmodified, adipose derived regenerative cells, isolated at point of care) in regenerative medicine. Cells 2020;9:1097. doi: 10.3390/cells9051097.

39. Kozlowska U, Krawczenko A, Futoma K, et al. Similarities and differences between mesenchymal stem/progenitor cells derived from various human tissues. World J Stem Cells 2019;11:347-374. doi: 10.4252/wjsc.v11.i6.347.

40. Srivastava M, Ahlawat N, Srivastava A. Amniotic fluid stem cells: a new era in regenerative medicine. J Obstet Gynaecol India 2018;68:15-9. doi: 10.1007/s13224-017-1034-z.

41. Higgins JPT, Thomas J, eds. Cochrane Handbook for Systematic Reviews of Interventions. 2nd ed. Hoboken, NJ: John Wiley \& Sons; 2008. 
42. Heitjan DF. Ignorability and bias in clinical trials. Stat Med 1999;18:2421-34. doi: 10.1002/(sici)10970258(19990915/30)18:17/18<2421::aid-sim266>3.0.co;2-r.

43. Panés J, García-Olmo D, Van Assche G, et al. Expanded allogeneic adipose-derived mesenchymal stem cells (Cx601) for complex perianal fistulas in Crohn's disease: a phase 3 randomised, doubleblind controlled trial. Lancet 2016;388:1281-90. doi: 10.1016/S0140-6736(16)31203-X.

44. Álvaro-Gracia JM, Jover JA, García-Vicuña R, et al. Intravenous administration of expanded allogeneic adipose-derived mesenchymal stem cells in refractory rheumatoid arthritis (Cx611): results of a multicentre, dose escalation, randomised, single-blind, placebo-controlled phase Ib/IIa clinical trial. Ann Rheum Dis
2017;76:196-202. doi: 10.1136/annrheumdis-2015-208918.

45. Kastrup J, Haack-Sørensen M, Juhl M, et al. Cryopreserved off-theshelf allogeneic adipose-derived stromal cells for therapy in patients with ischemic heart disease and heart failure-a safety study. Stem Cells Transl Med 2017;6:1963-71. doi: 10.1002/sctm.17-0040.

46. Marks PW, Witten CM, Califf RM. Clarifying stem-cell therapy's benefits and risks. $N$ Engl $J$ Med.2017;376:1007-9. doi: 10.1056/NEJMp1613723.

47. Eder C, Schmidt-Bleek K, Geissler S, et al. Mesenchymal stromal cell and bone marrow concentrate therapies for musculoskeletal indications: a concise review of current literature. Mol Biol Rep 2020;47:4789-814. doi: 10.1007/s11033-020-05428-0 


\author{
Methodological flaws in meta-analyses of clinical studies on treatment of knee \\ osteoarthritis with stem cells \\ Christoph Schmitz, Christopher Alt, David A. Pearce, \\ John P. Furia, Nicola Maffulli, Eckhard U. Alt
}

\title{
Supplementary Material
}

Note: the reference numbers $15-33$ refer to references in the main text, whereas the reference numbers S1-S187 refer to references provided in this Supplementary Material. 
Table S1 | Conclusions of 19 meta-analyses (published between January 2020 and July 2021) of studies in which treatment of primary knee osteoarthritis with different types of stem cells were investigated.

Abbreviations: $\mathrm{AD}=$ adipose-derived; $\mathrm{BM}=$ bone marrow-derived; $\mathrm{BMAC}=$ bone marrow aspirate concentrate; HA = hyaluronic acid; KOOS = Knee Injury and Osteoarthritis Outcome Score; MSCs = mesenchymal stem cells; $\mathrm{OA}=$ osteoarthritis; $\mathrm{PRP}=$ platelet rich plasma; $\mathrm{R}=$ reference number (note that the reference numbers provided in this table refer to the reference numbers in the main text); $\mathrm{SVF}=$ stromal vascular fraction; WOMAC $=$ Western Ontario and McMaster Universities Osteoarthritis Index.

\section{R Conclusion (original quotes taken from the abstracts of the cited papers)}

\section{Meta-analyses that demonstrated efficacy of treating pkOA with stem cells}

"There are significant therapeutic effects on joint function, symptoms, and no permanent adverse
effect has been found after stem cell treatment. It is promising to apply intro-articular injection
of stem cells for OA to clinical application."
"Based on the current studies, our results suggested that MSCs were a promising option for the
treatment of patients with knee OA."
"Intra-articular injection of MSCs is effective and safe to relieve pain and improve motor function
of patients with knee OA in a short term which is different to conclusions of previous study."
"We demonstrated that MSC treatment could significantly decrease visual analog scale in a 12-
month follow-up study compared with controls (p < 0.001). MSC therapy also showed significant
decreases in WOMAC scores after the 6-month follow-up (p < 0.001). MSC therapy showed no
difference compared with controls (p > 0.05) in adverse events. We suggest that MSC therapy
could serve as an effective and safe therapy for clinical application in OA treatment."
"Stem cell therapy is certainly superior to traditional treatments in the conservative treatment of
knee OA; it considerably reduces pain with no obvious additional side effects."
" MSCs relieve pain, stiffness, and dysfunction due to OA better than PRP, HA, and GCs and are
not statistically correlated with greater safety concerns

Meta-analyses that demonstrated superiority of autologous, adipose-derived stem cells over other types of stem cells in treatment of knee $O A$

"These findings suggested that MSCs are effective in the treatment of knee OA. AD-MSCs might
be the most effective for relieving pain, and umbilical cord-derived mesenchymal stem cells
might be the most effective for improving function. However, the current evidence does not
support the use of MSCs for improving cartilage repair in knee OA patients."
"Overall, MSC-based cell therapy is a relatively safe treatment that holds great potential for OA,
evidenced by a positive effect on pain and knee function. Using low-dose (25 million) and
adipose-derived stem cells is likely to achieve better results."
"A single BMAC or SVF injection into the knee joint of patients with OA resulted in symptomatic
improvement at short-term follow-up. However, SVF seemed to be more effective than did
BMAC in the reduction of knee pain."
"Our analysis establishes the efficacy, safety, and superiority of AD-MSC transplantation,
compared to BM-MSC, in the management of osteoarthritis of knee from available literature."
"The therapeutic effect of AD-MSCs on knee OA was more effective than that of BM-MSCs." 
Table S1 (cont.)

\begin{tabular}{ll}
\hline $\mathbf{R}$ & Conclusion \\
\hline $\begin{array}{l}\text { Meta-analysis that demonstrated efficacy of treating pkOA with autologous, adipose-derived stem } \\
\text { cells, without comparison with other types of stem cells }\end{array}$ \\
\hline $26 \quad \begin{array}{l}\text { "Pooled analysis revealed that cell-based treatments definitively improve WOMAC scores, post } \\
\text { treatment. These improvements increased with time. The studies in this meta-analysis have } \\
\text { established the safety and efficacy of both AD-MSC therapy and SVF therapy for knee OA in } \\
\text { old adults and show that they reduce pain and improve knee function in symptomatic knee OA } \\
\text { suggesting that they may be effective therapies to improve mobility in an aging population." } \\
\text { "During } 6 \text { months of follow-up, AD-MSCs relieved pain the best; LP-PRP was most effective for } \\
\text { functional improvement. During the 12-month follow-up, both AD-MSCs and LP-PRP showed } \\
\text { potential clinical pain relief effects; functional improvement was achieved with LP-PRP." }\end{array}$ \\
\hline
\end{tabular}

Meta-analysis that demonstrated superiority of autologous, bone marrow-derived stem cells over autologous, adipose-derived stem cells in treatment of pkOA

28 "Intra-articular injections of MSCs without any adjuvant therapies improves pain and function for osteoarthritis. Significantly better outcomes were obtained with the use of bone marrow MSCs as compared with adipose MSCs and with the use of cultured MSCs as opposed to uncultured MSCs."

Meta-analysis that demonstrated efficacy of treating pkOA with autologous, bone marrow-derived stem cells, without comparison with other types of stem cells

29 "Intra-articular injection of culture-expanded MSCs without adjuvant surgery can improve pain for patients experiencing knee osteoarthritis at short-term follow-up (6-12 months)."

Meta-analysis that demonstrated efficacy of treating pkOA with allogeneic stem cells

30 "Cell-based therapy had a better effect on KOOS improvement and pain relief without safety concerns. However, cell-based therapy did not show a benefit in terms of the WOMAC. Allogeneic cells might have advantages compared to controls in the WOMAC and KOOS scores."

Meta-analysis that demonstrated efficacy of treating pkOA with stem cells only in conjunction with surgery

31 "The pooled standardized mean difference from meta-analyses showed statistically significant effects of MSC on self-reported physical function but not self-reported pain. MSCs provided functional benefit only in patients who underwent concomitant surgery."

Meta-analysis that demonstrated lack of efficacy of treating pkOA with stem cells

32 "The ranking statistics like surface under the cumulative ranking curve values of our network meta-analysis support the use of steroids and HA for appropriate patients with knee OA. For pain relief and adverse events, steroids are most likely the best treatment, followed by HA. Single PRP, multiple PRP, and adipose MSC interventions do not result in a relevant reduction of joint pain nor improvement of joint function compared with placebo."

33 "Intra-articular MSC injection was not found to be superior to placebo in pain relief and functional improvement for patients with symptomatic knee OA." 
Table S2 | Categories of studies included in the 19 meta-analyses summarized in Table S1 of studies in which treatment of primary knee osteoarthritis (pkOA) with stem cells was investigated.

Abbreviations: $\mathrm{Ca}=$ category; $\mathrm{CS}=$ corticosteroid; $\mathrm{HA}=$ hyaluronic acid; i.a. $=$ intra-articular; $\mathrm{N}_{\text {all }}=$ number of studies among all studies included in the 19 assessed meta-analyses; PRP = platelet rich plasma.

\begin{tabular}{|c|c|c|}
\hline $\mathbf{C a}$ & $\mathbf{N}_{\text {all }}$ & Description \\
\hline I & 8 & $\begin{array}{l}\text { Treatment of pkOA with i.a. injection of stem cells as the sole treatment (not considering } \\
\text { rehabilitation), compared with i.a. injection of saline or sham treatment as control. }\end{array}$ \\
\hline II & 6 & $\begin{array}{l}\text { Treatment of pkOA with i.a. injection of stem cells as the sole treatment (not considering } \\
\text { rehabilitation), compared with i.a. injection of respectively PRP, CS or HA as control. }\end{array}$ \\
\hline III & 10 & $\begin{array}{l}\text { Treatment of pkOA with i.a. injection of stem cells as the sole treatment (not considering } \\
\text { rehabilitation), compared with other treatments than those in Categories I and II as } \\
\text { control. }\end{array}$ \\
\hline IV & 12 & $\begin{array}{l}\text { Treatment of pkOA with combinations of stem cells and other modalities, compared with } \\
\text { sham treatment or other treatments as control. }\end{array}$ \\
\hline V & 13 & $\begin{array}{l}\text { Treatment of pkOA with combinations of stem cells with or without other modalities, } \\
\text { without control group (case series or case reports). }\end{array}$ \\
\hline VI & 4 & $\begin{array}{l}\text { Treatment of focal chondral, osteochondral or meniscal chondral lesions with stem cells } \\
\text { as the sole treatment (not considering rehabilitation) or combinations of stem cells and } \\
\text { other modalities, with or without other treatments as control. }\end{array}$ \\
\hline VII & 1 & Treatment of pkOA without stem cells \\
\hline $\mathrm{N}$ & 2 & $\begin{array}{l}\text { Study not listed in PubMed, Embase, Web of Science, Cochrane Library and Googe } \\
\text { Scholar }\end{array}$ \\
\hline Sum & 56 & \\
\hline
\end{tabular}


Table S3 | Types of studies included in the meta-analyses summarized in Table S1 of studies in which treatment of primary knee osteoarthritis (pkOA) with stem cells was investigated.

Abbreviations: $\mathrm{Ca}=$ category; $\mathrm{N}_{\text {all }}=$ number of studies among all studies included in the 19 assessed metaanalyses; $\mathrm{N}_{\mathrm{pkOA}}=$ number of studies among those studies included in the 19 assessed meta-analyses that addressed pkOA.

\begin{tabular}{cccl}
\hline Ca & $\mathbf{N}_{\text {all }}$ & $\mathbf{N}_{\mathbf{p k O A}}$ & Description \\
\hline $\mathrm{a}$ & 29 & 25 & Randomized controlled trials (RCTs) \\
$\mathrm{b}$ & 3 & 3 & RCTs with the contralateral knee as internal control \\
$\mathrm{c}$ & 7 & 7 & Prospective cohort studies \\
$\mathrm{d}$ & 2 & 2 & Retrospective cohort studies \\
$\mathrm{e}$ & 13 & 13 & Case series with more than one subject \\
$\mathrm{f}$ & 0 & 0 & Case reports with only one subject \\
\hline $\mathrm{N}$ & 2 & 2 & Study not listed in PubMed, Embase, Web of Science, Cochrane Library and \\
& & & Googe Scholar \\
\hline Sum & 56 & 52 & \\
\hline
\end{tabular}


Table S4 | Cell types used in those studies included in the meta-analyses summarized in Table S1 in which treatment of primary knee osteoarthritis with stem cells was investigated.

Abbreviations: Auto $=$ autologous cells; Allo $=$ allogeneic cells; $\mathrm{C}=$ cells; $\mathrm{N}_{\text {all }}=$ number of studies among all studies included in the 19 assessed meta-analyses; $\mathrm{N}_{\mathrm{pkOA}}=$ number of studies among those studies included in the 19 assessed meta-analyses that addressed pkOA.

\begin{tabular}{|c|c|c|c|c|}
\hline Cell type & $\mathbf{C}$ & $\mathbf{N}_{\text {all }}$ & $\mathbf{N}_{\text {pkOA }}$ & Description \\
\hline ADRCs & Auto & 9 & 9 & Autologous, adipose-derived regenerative cells \\
\hline ADSCs & Auto & 11 & 11 & $\begin{array}{l}\text { Autologous, adipose-derived stem cells (obtained by culturing } \\
\text { ADRCs) }\end{array}$ \\
\hline ADSCs & Allo & 1 & 1 & Allogeneic, adipose-derived stem cells \\
\hline MFF & Auto & 4 & 4 & Autologous, micro-fragmented fat (from liposuction) \\
\hline CLL & Auto & 1 & 1 & Autologous, centrifuged liposuction liquid \\
\hline BMAC & Auto & 7 & 7 & Autologous bone marrow concentrate \\
\hline BM-MSCs & Auto & 10 & 9 & Autologous, bone marrow-derived mesenchymal stem cells \\
\hline BM-MSCs & Allo & 3 & 2 & Allogeneic, bone marrow-derived mesenchymal stromal cells \\
\hline S-MSCs & Auto & 1 & 0 & Autologous, matrix-induced MSCs from synovia \\
\hline Ch-TGFß & Allo & 2 & 2 & $\begin{array}{l}\text { Allogeneic chondrocytes that overexpress transcription } \\
\text { growth factor beta }\end{array}$ \\
\hline hUC-MSCs & Allo & 2 & 2 & Allogeneic, human umbilical cord-derived MSCs \\
\hline P-MSCs & Allo & 1 & 1 & Allogeneic, placental MSCs \\
\hline pBSCs & Auto & 1 & 0 & Autologous, activated peripheral blood stem cells \\
\hline No cells & Allo & 1 & 1 & Allogeneic amniotic fluid \\
\hline Sum & & 54 & 50 & \\
\hline
\end{tabular}


Table S5 | Type of analysis performed in the meta-analyses summarized in Table S1 in which treatment of primary knee osteoarthritis with stem cells was investigated.

Abbreviation: $\mathrm{Cl}=$ Class; $\mathrm{N}=$ number of meta-analyses.

\begin{tabular}{ccl}
\hline $\mathbf{C l}$ & $\mathbf{N}$ & Description \\
\hline 1 & 12 & $\begin{array}{l}\text { Meta-analysis of studies in which treatment of pkOA with stem cells was compared } \\
\text { with placebo treatment (or studies in which treatment of pkOA with stem cells plus } \\
\text { concomitant therapy was compared with the concomitant therapy alone, } \\
\text { respectively). } \\
\text { Meta-analysis in which only endpoints of the same patients before and after } \\
\text { treatment were compared. } \\
\text { Network meta-analysis that included only a small number of studies on treatment } \\
\text { of pkOA with stem cells and a much higher number of studies on treatment of } \\
\text { pkOA without stem cells. }\end{array}$ \\
\hline Sum & 19 & \\
\hline
\end{tabular}


Table S6 | Details of the studies included in the meta-analyses summarized in Table S1 in which treatment of primary knee osteoarthritis (pkOA) with different types of stem cells was investigated.

Abbreviations: $\mathrm{AD}=$ arthroscopic debridement; $\mathrm{BMC}=$ bone marrow concentrate; $\mathrm{C}=$ cells; $\mathrm{Ca}=$ category of study as shown in Table $\mathrm{S} 2 ; \mathrm{CM}$ = conservative management $\mathrm{CS}=$ corticosteroid; $\mathrm{HA}=$ hyaluronic acid; $\mathrm{HTO}=$ high tibial osteotomy; MACI = matrix-induced, autologous chondrocyte implant; $\mathrm{MF}=$ microfracture; $\mathrm{N}=$ number of meta-analyses in which the corresponding study was included; $\mathrm{OA}=$ oral acetaminophen; $\mathrm{PPP}=$ platelet poor plasma; $\mathrm{PRP}=$ platelet rich plasma; $\mathrm{R}=$ reference number; $\mathrm{RS}=$ Ringer solution; $\mathrm{T}=$ Type of study as shown in Table $\mathrm{S} 3$; $\mathrm{U}=$ unknown; $\mathrm{x}$ ADRCs = autologous, adipose-derived regenerative cells; $\mathrm{x}$ ADSCs $=$ autologous, adipose-derived stem cells; $\mathrm{x}$ BMAC $=$ autologous bone marrow aspirate concentrate; $\mathrm{x}$ BM-MSCs = autologous, bone marrow-derived mesenchymal stem cells; $\mathrm{x}$ CLL = autologous, centrifuged liposuction liquid; $\mathrm{x}$ MFF = autologous, micro-fragmented fat; $\mathrm{x}$ pBSCs = autologous, activated peripheral blood stem cells; $y$ ADSCs = allogeneic, adipose-derived stem cells; y BM-MSCs = allogeneic, bone marrow-derived mesenchymal stromal cells; y Ch-TGF $\beta=$ allogeneic chondrocytes that overexpress transcription growth factor beta; y hUC-MSCs = allogeneic, human umbilical cord-derived MSCs; y P-MSCs = allogeneic, placental MSCs; $\bullet$ = study included in the corresponding meta-analysis.

\begin{tabular}{|c|c|c|c|c|c|c|c|c|c|c|c|c|c|c|c|c|c|c|c|c|c|c|c|c|c|c|c|}
\hline \multirow[b]{2}{*}{$\mathbf{R}$} & \multirow[b]{2}{*}{$\begin{array}{l}\mathbf{C} \\
\mathbf{a}\end{array}$} & \multirow[b]{2}{*}{$\mathbf{T}$} & \multirow[b]{2}{*}{ First author } & \multirow[b]{2}{*}{ Year } & \multirow[b]{2}{*}{ Cells } & \multirow[b]{2}{*}{$\mathbf{T}$} & \multirow[b]{2}{*}{ Control } & \multicolumn{20}{|c|}{ Reference no. in the main text } \\
\hline & & & & & & & & $\begin{array}{l}1 \\
5\end{array}$ & $\begin{array}{l}1 \\
6\end{array}$ & $\begin{array}{l}1 \\
7\end{array}$ & $\begin{array}{l}1 \\
8\end{array}$ & $\begin{array}{l}1 \\
9\end{array}$ & $\begin{array}{l}2 \\
0\end{array}$ & $\begin{array}{l}2 \\
1\end{array}$ & $\begin{array}{l}2 \\
2\end{array}$ & $\begin{array}{l}\mathbf{2} \\
\mathbf{3}\end{array}$ & $\begin{array}{l}2 \\
4\end{array}$ & $\begin{array}{l}2 \\
5\end{array}$ & $\begin{array}{l}2 \\
6\end{array}$ & $\begin{array}{l}2 \\
7\end{array}$ & $\begin{array}{l}2 \\
8\end{array}$ & $\begin{array}{l}2 \\
9\end{array}$ & $\begin{array}{l}\mathbf{3} \\
\mathbf{0}\end{array}$ & $\begin{array}{l}3 \\
1\end{array}$ & $\begin{array}{ll}\mathbf{3} & \mathbf{3} \\
\mathbf{2} & \mathbf{3}\end{array}$ & $\begin{array}{l}3 \\
3\end{array}$ & $\mathbf{N}$ \\
\hline S7 & 1 & $\mathrm{a}$ & Cherian & 2015 & y Ch-TGFß & $\mathrm{C}$ & Saline & & & & & & & & & & & & & & & & $\bullet$ & & & & 1 \\
\hline S13 & 1 & a & Gupta & 2016 & y BM-MSCs & $\mathrm{C}+\mathrm{HA}$ & $\mathrm{S}+\mathrm{HA}$ & & $\bullet$ & • & • & & & $\bullet$ & $\bullet$ & & $\bullet$ & $\bullet$ & & & & $\bullet$ & $\bullet$ & $\bullet$ & $\bullet$ & & 11 \\
\hline S8 & 1 & a & Emadedin & 2018 & x BM-MSCs & $\mathrm{C}$ & Saline & & $\bullet$ & $\bullet$ & & & • & $\bullet$ & $\bullet$ & & $\bullet$ & & & $\bullet$ & $\bullet$ & $\bullet$ & $\bullet$ & & $\bullet$ & & 11 \\
\hline S22 & 1 & a & Kim & 2018 & y Ch-TGFß & $\mathrm{C}$ & Saline & & & & & & & & & & & & & & & & $\bullet$ & $\bullet$ & & & 2 \\
\hline S26 & 1 & $\mathrm{a}$ & Kuah & 2018 & y ADSCs & $\mathrm{C}$ & Saline & & & $\bullet$ & $\bullet$ & $\bullet$ & & $\bullet$ & $\bullet$ & & $\bullet$ & & & & $\bullet$ & $\bullet$ & $\bullet$ & & $\bullet$ & & 10 \\
\hline S21 & 1 & $\mathrm{a}$ & Khalifeh Soltani & 2019 & y P-MSCs & $\mathrm{C}$ & Saline & & & $\bullet$ & & & & & & & & & & & & $\bullet$ & & & $\bullet$ & & 3 \\
\hline S28 & 1 & $\mathrm{a}$ & Lee & 2019 & $\mathrm{x}$ ADSCs & $\mathrm{C}$ & Saline & & & $\bullet$ & $\bullet$ & $\bullet$ & & $\bullet$ & & & $\bullet$ & & $\bullet$ & $\bullet$ & $\bullet$ & & $\bullet$ & $\bullet$ & $\bullet$ & & 11 \\
\hline S11 & 1 & $\mathrm{a}$ & Garza & 2020 & $\mathrm{x}$ ADRCs & $\mathrm{C}$ & $\mathrm{RS}$ & & & & & & & & & $\bullet$ & $\bullet$ & & $\bullet$ & & & & & & $\bullet$ & & 4 \\
\hline S51 & 2 & $\mathrm{a}$ & Vega & 2015 & y BM-MSCs & $\mathrm{C}$ & HA & $\bullet$ & $\bullet$ & $\bullet$ & $\bullet$ & $\bullet$ & & $\bullet$ & $\bullet$ & & $\bullet$ & $\bullet$ & & $\bullet$ & $\bullet$ & $\bullet$ & $\bullet$ & $\bullet$ & $\bullet$ & & 15 \\
\hline S52 & 2 & a & Wang & 2016 & y hUC-MSCs & $\mathrm{C}$ & HA & $\bullet$ & & & $\bullet$ & & & & & & & & & & & & $\bullet$ & & & & 3 \\
\hline $\mathrm{S} 12$ & 2 & $\mathrm{a}$ & Goncars & 2017 & x BMACBMAC & $\mathrm{C}$ & HA & & & & & & & & & & & & & & $\bullet$ & & & & & & 1 \\
\hline S29 & 2 & $\mathrm{a}$ & $\mathrm{Lu}$ & 2019 & $\mathrm{x}$ ADSCs & $\mathrm{C}$ & HA & $\bullet$ & & $\bullet$ & & & & & $\bullet$ & & $\bullet$ & $\bullet$ & & $\bullet$ & $\bullet$ & & $\bullet$ & $\bullet$ & & $\bullet$ & 11 \\
\hline S31 & 2 & $\mathrm{a}$ & Matas & 2019 & y hUC-MSCs & $\mathrm{C}$ & HA & $\bullet$ & & $\bullet$ & $\bullet$ & & $\bullet$ & $\bullet$ & $\bullet$ & & & & & & & & $\bullet$ & & $\bullet$ & & 8 \\
\hline S3 & 2 & $\mathrm{a}$ & Anz & 2020 & $\mathrm{x}$ BMACBMAC & $\mathrm{C}$ & PRP & & & & & & & & & $\bullet$ & & & & & & & & & & & 1 \\
\hline S10 & 3 & $\mathrm{a}$ & Garay-Mendoza & 2018 & x BMACBMAC & $\mathrm{C}$ & $\mathrm{OA}$ & & & & & & & & & $\bullet$ & $\bullet$ & & & & $\bullet$ & & & & & & 3 \\
\hline S44 & 3 & a & Song & 2018 & $\mathrm{x}$ ADSCs & $\mathrm{C}$ & $\mathrm{C}$ & & & & $\bullet$ & & & & & & & & $\bullet$ & & & & & & & & 2 \\
\hline S9 & 3 & a & Freitag & 2019 & $\mathrm{x}$ ADSCs & $\mathrm{C}$ & $\mathrm{CM}$ & & & $\bullet$ & & & & & & & $\bullet$ & $\bullet$ & $\bullet$ & & $\bullet$ & & $\bullet$ & & $\bullet$ & & 7 \\
\hline S4 & 3 & a & Bastos & 2020 & x BM-MSCs & $\mathrm{C}$ & $\mathrm{C}+\mathrm{PRP}+\mathrm{CS}$ & & & & & & $\bullet$ & & & & $\bullet$ & & & & $\bullet$ & & & & & & 2 \\
\hline $\mathrm{S} 20$ & 3 & $\mathrm{c}$ & $\mathrm{JO}$ & 2014 & $\mathrm{x}$ ADSCs & $\mathrm{C}$ & $\mathrm{C}$ & & & & & & & & & & & & $\bullet$ & & & & & & & & 1 \\
\hline S36 & 3 & $\mathrm{c}$ & Pers & 2016 & $\mathrm{x}$ ADSCs & $\mathrm{C}$ & $\mathrm{C}$ & & & & $\bullet$ & & & & & & & & $\bullet$ & & & & & & & & 2 \\
\hline S19 & 3 & $\mathrm{c}$ & Jo & 2017 & x ADSCs & $\mathrm{C}$ & $\mathrm{C}$ & & & & $\bullet$ & & & & & & & & $\bullet$ & & & & & & & & 2 \\
\hline S35 & 3 & $\mathrm{c}$ & Pers & 2018 & $\mathrm{x}$ ADSCs & $\mathrm{C}$ & $\mathrm{C}$ & & & & & & & & & & & & & & $\bullet$ & & & & & & 1 \\
\hline $\mathrm{S} 32$ & 3 & $\mathrm{~d}$ & Mautner & 2019 & $\mathrm{x}$ MFF & $\mathrm{C}$ & BMACBMAC & & & & & & & & & $\bullet$ & & & & & & & & & & & 1 \\
\hline
\end{tabular}


Table S6 (cont.)

\begin{tabular}{|c|c|c|c|c|c|c|c|c|c|c|c|c|c|c|c|c|c|c|c|c|c|c|}
\hline \multirow{3}{*}{$\mathbf{R}$} & \multirow{3}{*}{$\begin{array}{l}\mathbf{C} \\
\mathbf{a}\end{array}$} & \multirow{3}{*}{$\mathbf{T}$} & \multirow{3}{*}{ First author } & \multirow{3}{*}{ Year } & \multirow{3}{*}{ Cells } & \multirow{3}{*}{$\mathbf{T}$} & \multirow{3}{*}{ Control } & \multicolumn{14}{|c|}{ Reference no. in the main text } & \multirow{3}{*}{$\mathbf{N}$} \\
\hline & & & & & & & & 1 & 1 & 1 & 1 & 12 & 22 & 2 & 22 & 2 & 2 & 2 & 2 & 3 & 33 & \\
\hline & & & & & & & & 5 & 67 & 78 & 9 & 90 & 12 & 3 & 45 & 6 & 7 & 8 & 9 & 0 & 12 & \\
\hline S54 & 3 & $\mathrm{~d}$ & Yokota & 2019 & $\mathrm{x}$ ADRCs & $\mathrm{C}$ & $\mathrm{C}$ & & & & & & & $\bullet$ & & $\bullet$ & & & & & & 2 \\
\hline S50 & 4 & $\mathrm{a}$ & Varma & 2010 & x BM-MSCs & $\mathrm{C}+\mathrm{AD}$ & $\mathrm{AD}$ & & $\bullet$ & & & & & & & & & & & & & 1 \\
\hline S53 & 4 & $\mathrm{a}$ & Wong & 2013 & x BM-MSCs & $\begin{array}{l}\mathrm{C}+\mathrm{MF}+\mathrm{HTO} \\
+\mathrm{HA}\end{array}$ & $\begin{array}{l}\mathrm{MF}+\mathrm{HTO}+ \\
\mathrm{HA}\end{array}$ & & & & & $\bullet$ & & & - $\bullet$ & & & & & & & 3 \\
\hline S25 & 4 & $\mathrm{a}$ & Koh & 2014 & x ADRCs & $\begin{array}{l}\mathrm{C}+\mathrm{AD}+\mathrm{HTO} \\
+\mathrm{PRP}\end{array}$ & $\begin{array}{l}\mathrm{AD}+\mathrm{HTO}+ \\
\mathrm{PRP}\end{array}$ & & & & & & & & $\bullet$ & & & & & & $\bullet$ & 2 \\
\hline S27 & 4 & $\mathrm{a}$ & Lamo-Espinosa & 2016 & x BM-MSCs & $\mathrm{C}+\mathrm{HA}$ & $\mathrm{HA}$ & $\bullet$ & $\bullet$ & $\bullet$ & & $\bullet$ & $\bullet \bullet$ & & $\bullet$ & & $\bullet$ & & $\bullet$ & $\bullet$ & $\bullet$ & 12 \\
\hline S5 & 4 & $\mathrm{a}$ & Bastos & 2018 & x BM-MSCs & $\mathrm{C}+\mathrm{PRP}$ & $\mathrm{C}$ & & & • & & & & & & & & & & - & & 3 \\
\hline S56 & 4 & $\mathrm{a}$ & Zhang & 2018 & $\mathrm{x}$ ADSCs & $\mathrm{C}+\mathrm{HA}$ & $\mathrm{C}$ & & & & & & & & & & $\bullet$ & & & & & 1 \\
\hline S41 & 4 & $\mathrm{~b}$ & Shapiro & 2017 & X BMACBMAC & $\mathrm{C}+\mathrm{PPP}$ & Saline & & & & & $\bullet$ & & & & & & & & & & 1 \\
\hline S16 & 4 & $\mathrm{~b}$ & Hong & 2019 & $\mathrm{x}$ ADRCs & $\mathrm{C}+\mathrm{AD}$ & $\mathrm{HA}+\mathrm{AD}$ & & & & & & & & $\bullet$ & $\bullet$ & & & & & - • & 4 \\
\hline S40 & 4 & $\mathrm{~b}$ & Shapiro & 2019 & X BMACBMAC & $\mathrm{C}+\mathrm{PPP}$ & Saline & & & & & & & & & & & & & & $\bullet$ & 1 \\
\hline $\mathrm{S} 23$ & 4 & $\mathrm{c}$ & Koh & 2012 & x ADSCs & $C+A D+P R P$ & $\mathrm{AD}+\mathrm{PRP}$ & & & $\bullet$ & 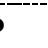 & & & & $\bullet$ & & & & & & $\bullet$ & 4 \\
\hline S33 & 4 & $\mathrm{c}$ & Nguyen & 2017 & $\mathrm{x}$ ADRCs & $\begin{array}{l}\mathrm{C}+\mathrm{AD}+\mathrm{MF}+ \\
\mathrm{PRP}\end{array}$ & $\mathrm{AD}+\mathrm{MF}$ & & & & & & & & & $\bullet$ & & & & & & 1 \\
\hline S47 & 4 & c & Tran & 2019 & $\mathrm{x}$ ADRCs & $\mathrm{C}+\mathrm{AD}+\mathrm{MF}$ & $\mathrm{AD}+\mathrm{MF}$ & & & & & & & & $\bullet$ & $\bullet$ & & & & & & 2 \\
\hline S24 & 5 & $\mathrm{e}$ & Koh & 2013 & $\mathrm{x}$ ADRCs & $\mathrm{C}+\mathrm{PRP}+\mathrm{HA}$ & None & & & & & & & & & $\bullet$ & & & & & & 1 \\
\hline S34 & 5 & $\mathrm{e}$ & Orozco & 2013 & x BM-MSCs & $\mathrm{C}$ & None & & & & & & & & & & & $\bullet$ & & & & 1 \\
\hline S43 & 5 & $\mathrm{e}$ & Soler & 2016 & x BM-MSCs & $\mathrm{C}$ & None & & & & & & & & & & & $\bullet$ & & & & 1 \\
\hline $\mathrm{S} 2$ & 5 & $\mathrm{e}$ & Al-Najar & 2017 & x BM-MSCs & $\mathrm{C}$ & None & & & & & & & & & & & $\bullet$ & & & & 1 \\
\hline S18 & 5 & $\mathrm{e}$ & Hudetz & 2017 & $\mathrm{x} \mathrm{MFF}$ & $\mathrm{C}$ & None & & & & & & & & & & & $\bullet$ & & & & 1 \\
\hline S55 & 5 & $\mathrm{e}$ & Yokota & 2017 & $\mathrm{x}$ ADRCs & $\mathrm{C}$ & None & & & & & & & $\bullet$ & & & & $\bullet$ & & & & 2 \\
\hline S42 & 5 & $\mathrm{e}$ & Shaw & 2018 & x BMACBMAC & $\mathrm{C}$ & None & & & & & & & & & & & $\bullet$ & & & & 1 \\
\hline S45 & 5 & $\mathrm{e}$ & Spasovski & 2018 & $\mathrm{x}$ ADSCs & $\mathrm{C}$ & None & & & $\bullet$ & 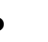 & & & & & $\bullet$ & & & & & & 2 \\
\hline S46 & 5 & $\mathrm{e}$ & Themistocleous & 2018 & x BMACBMAC & $\mathrm{C}$ & None & & & & & & & $\bullet$ & & & & & & & & 1 \\
\hline S17 & 5 & $\mathrm{e}$ & Hudetz & 2019 & x MFF & $\mathrm{C}$ & None & & & & & & & $\bullet$ & & $\bullet$ & & $\bullet$ & & & & 3 \\
\hline S37 & 5 & $\mathrm{e}$ & Roato & 2019 & x CLL & $\mathrm{C}+\mathrm{AD}$ & None & & & & & & & & & $\bullet$ & & & & & & 1 \\
\hline S39 & 5 & $\mathrm{e}$ & Schiavone Panni & 2019 & $\mathrm{x}$ MFF & $C+A D$ & None & & & & & & & & & $\bullet$ & & & & & & 1 \\
\hline $\mathrm{S} 48$ & 5 & $\mathrm{e}$ & Tsubosaka & 2020 & $\mathrm{x}$ ADRCs & C & None & & & & & & & $\bullet$ & & & & $\bullet$ & & & & 2 \\
\hline S38 & 6 & $\mathrm{a}$ & Saw & 2013 & $\mathrm{xpBSCs}$ & $\mathrm{C}+\mathrm{MF}+\mathrm{HA}$ & $\mathrm{HA}$ & & & & & $\bullet$ & & & & & & & & & & 1 \\
\hline $\mathrm{S} 1$ & 6 & $\mathrm{a}$ & Akgun & 2015 & x S-MSCs & $\mathrm{C}$ in $\mathrm{Sc}+\mathrm{AD}$ & MACI & & & & & & & & & & & & & & $\bullet$ & 1 \\
\hline S15 & 6 & $\mathrm{a}$ & Hashimoto & 2019 & x BM-MSCs & $\mathrm{C}+\mathrm{MF}$ & MF & & & & & & & & & & & & & & $\bullet$ & 1 \\
\hline S49 & 6 & $\mathrm{a}$ & Vangsness & 2014 & y BM-MSCs & & & & $\bullet$ & & & & & & - $\bullet$ & & & & & & & 3 \\
\hline S6 & 1 & $\mathrm{a}$ & Bhattacharia & 2010 & - & & & & & & & $\bullet$ & & & & & & & & & & 1 \\
\hline S30 & & & Lv & 2015 & $\mathrm{U}$ & & & & & & & & & & $\bullet$ & & & & & & & 1 \\
\hline $\mathrm{S} 14$ & & & $\mathrm{Ha}$ & 2018 & $\mathrm{U}$ & & & & • & & & & & & & & & & & & & 1 \\
\hline
\end{tabular}


Table S7 | Details of the sub-analyses performed in a meta-analysis by Jiang et al. ${ }^{28}$.

Abbreviations: allo = allogeneic cells; auto = autologous cells; $\mathrm{C}=$ category of study as outlined in Table S2; $\mathrm{O}=$ origin of cells; $\mathrm{QC}=$ quality criteria (outlined in Table 1 in the main text; a point indicates that the corresponding quality criterion was fulfilled); $\mathrm{R}=$ reference number; $\mathrm{T}=$ type of study as outlined in Table S3; Y = year of publication. The abbreviations of the cell types and treatments are provided in Table S6.

WOMAC Total score reported at 6 months post treatment

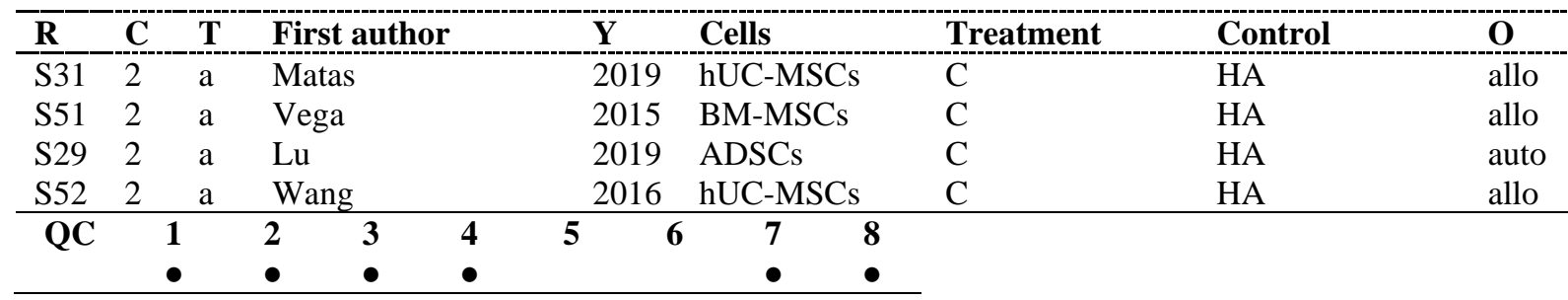

WOMAC Total score reported at 12 months post treatment

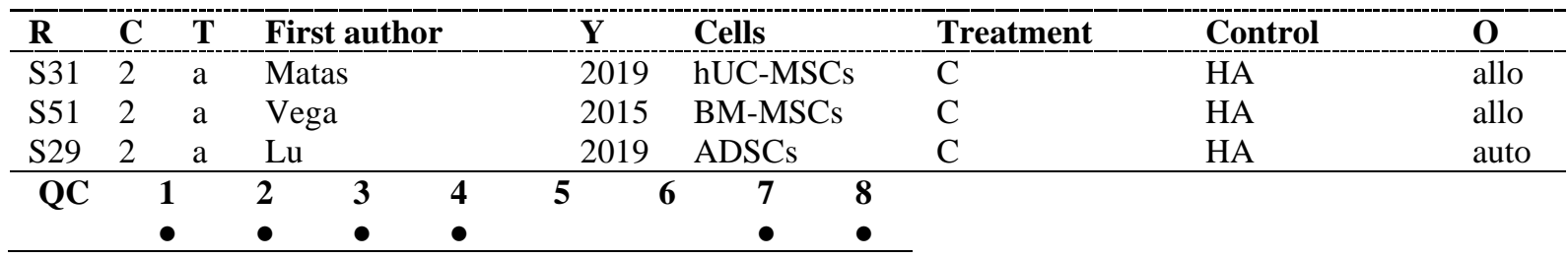

\section{WOMAC Pain score reported at 6 months post treatment}

\begin{tabular}{|c|c|c|c|c|c|c|c|c|}
\hline $\mathbf{R}$ & $\mathbf{C}$ & $\mathbf{T}$ & First author & $\mathbf{Y}$ & Cells & Treatment & Control & $\mathbf{O}$ \\
\hline S27 & 4 & $\mathrm{a}$ & Lamo-Espinosa & 2016 & BM-MSCs & $\mathrm{C}+\mathrm{HA}$ & $\mathrm{HA}$ & auto \\
\hline S29 & 2 & $\mathrm{a}$ & $\mathrm{Lu}$ & 2019 & ADSCs & $\mathrm{C}$ & HA & auto \\
\hline S31 & 2 & $\mathrm{a}$ & Matas & 2019 & hUC-MSCs & $\mathrm{C}$ & HA & allo \\
\hline S51 & 2 & $\mathrm{a}$ & Vega & 2015 & BM-MSCs & $\mathrm{C}$ & HA & allo \\
\hline QC & & & $\begin{array}{ll}2 & 3 \\
- & \bullet\end{array}$ & 56 & $\begin{array}{ll}7 & 8 \\
\bullet & \end{array}$ & & & \\
\hline
\end{tabular}

\section{WOMAC Stiffness score reported at 6 months post treatment}

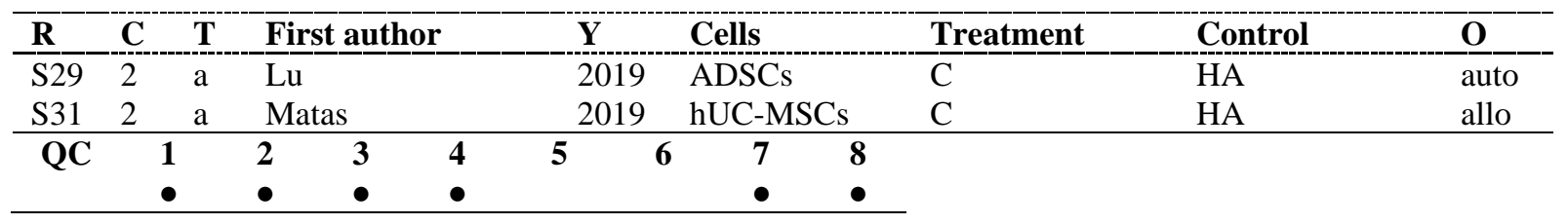


WOMAC Stiffness score reported at 12 months post treatment

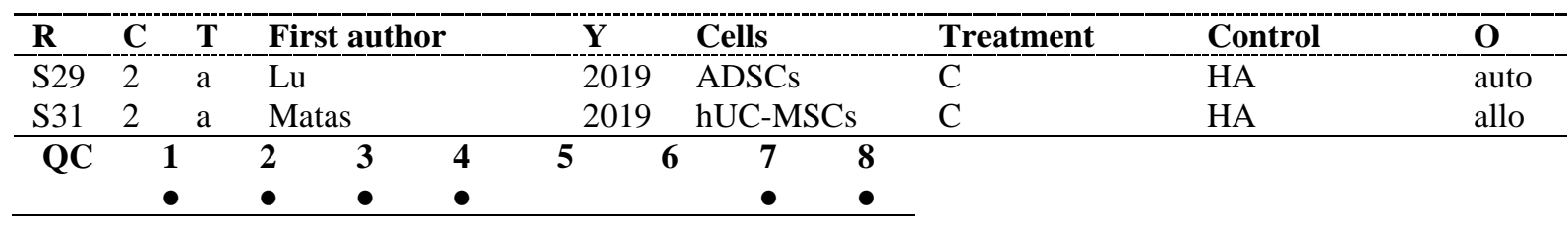

WOMAC Function score reported at 6 months post treatment

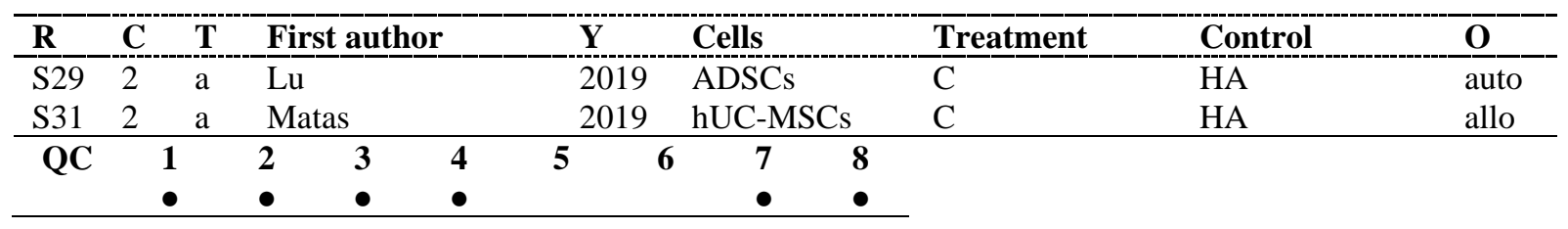

WOMAC Function score reported at 12 months post treatment

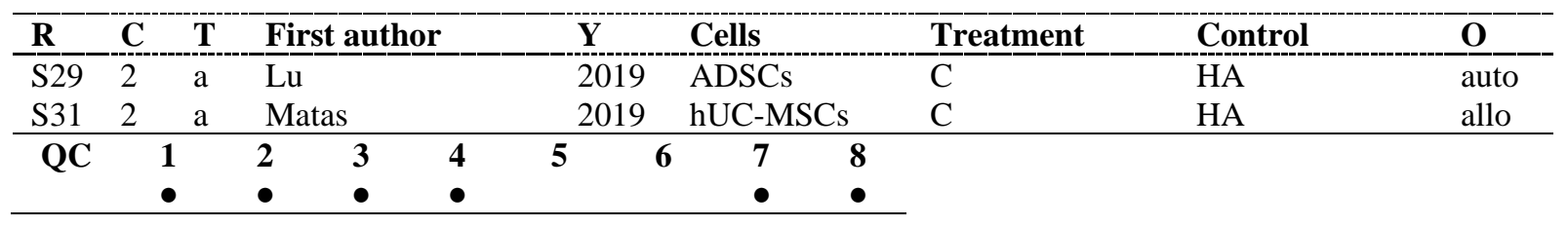

\begin{tabular}{|c|c|c|c|c|c|c|c|c|}
\hline $\mathbf{R}$ & $\mathrm{C}$ & $\mathbf{T}$ & First author & $\mathbf{Y}$ & Cells & Treatment & Control & $\mathbf{0}$ \\
\hline $\mathrm{S} 27$ & 4 & a & Lamo-Espinosa & 2016 & BM-MSCs & $\mathrm{C}+\mathrm{HA}$ & $\mathrm{HA}$ & auto \\
\hline S29 & 2 & $\mathrm{a}$ & $\mathrm{Lu}$ & 2019 & ADSCs & $\mathrm{C}$ & HA & auto \\
\hline S31 & 2 & $\mathrm{a}$ & Matas & 2019 & hUC-MSCs & $\mathrm{C}$ & HA & allo \\
\hline S51 & 2 & $\mathrm{a}$ & Vega & 2015 & BM-MSCs & $\mathrm{C}$ & HA & allo \\
\hline $\mathbf{Q C}$ & & & $\begin{array}{ll}2 & 3 \\
\bullet & \bullet\end{array}$ & $5 \quad 6$ & $\begin{array}{ll}7 \\
\bullet\end{array}$ & & & \\
\hline
\end{tabular}

VAS pain score reported at 12 months post treatment

\begin{tabular}{|c|c|c|c|c|c|c|c|c|}
\hline $\mathbf{R}$ & $\mathbf{C}$ & $\mathbf{T}$ & First author & $\mathbf{Y}$ & Cells & Treatment & Control & $\mathbf{O}$ \\
\hline S27 & 4 & $\mathrm{a}$ & Lamo-Espinosa & 2016 & BM-MSCs & $\mathrm{C}+\mathrm{HA}$ & $\mathrm{HA}$ & auto \\
\hline S29 & 2 & $\mathrm{a}$ & $\mathrm{Lu}$ & 2019 & $\mathrm{ADSCs}$ & $\mathrm{C}$ & HA & auto \\
\hline S31 & 2 & $\mathrm{a}$ & Matas & 2019 & hUC-MSCs & $\mathrm{C}$ & HA & allo \\
\hline S51 & 2 & $\mathrm{a}$ & Vega & 2015 & BM-MSCs & $\mathrm{C}$ & HA & allo \\
\hline QC & & & 23 & $5 \quad 6$ & $\begin{array}{ll}7 & 8\end{array}$ & & & \\
\hline & & & • & & $\bullet$ & & & \\
\hline
\end{tabular}


Whole-Organ Magnetic Resonance Imaging (WORMS) score reported at 6 months post treatment

\begin{tabular}{|c|c|c|c|c|c|c|c|c|c|c|c|}
\hline $\mathbf{R}$ & $\mathbf{C}$ & $\mathbf{T}$ & & au & & & & Cells & Treatment & Control & $\mathbf{O}$ \\
\hline $\mathrm{S} 27$ & 4 & $\mathrm{a}$ & & $-\mathrm{Es}$ & osa & & & BM-MSCs & $\mathrm{C}+\mathrm{HA}$ & $\mathrm{HA}$ & auto \\
\hline S31 & 2 & $\mathrm{a}$ & & & & & & hUC-MSCs & $\mathrm{C}$ & HA & allo \\
\hline QC & & & 2 & $\begin{array}{l}3 \\
\bullet\end{array}$ & $\begin{array}{l}4 \\
\bullet\end{array}$ & 5 & 6 & $\begin{array}{ll}7 & 8 \\
\bullet & \bullet \\
\end{array}$ & & & \\
\hline
\end{tabular}

WORMS score reported at 12 months post treatment

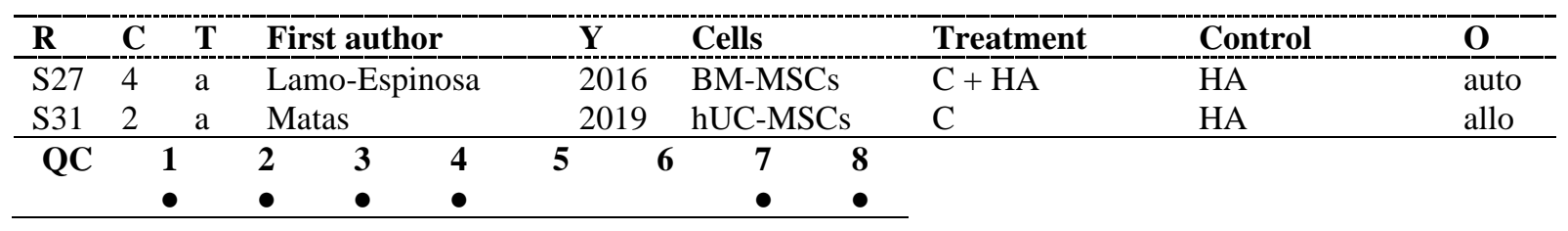

LEQUESNE score reported at 6 months post treatment

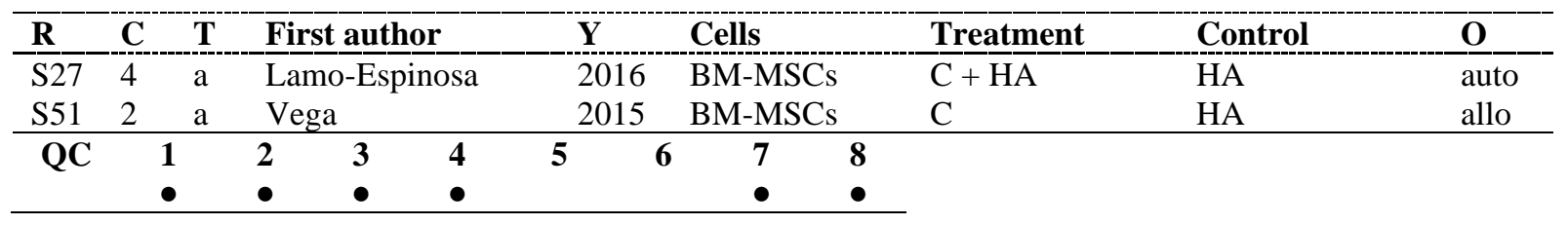

\section{LEQUESNE score reported at 12 months post treatment}

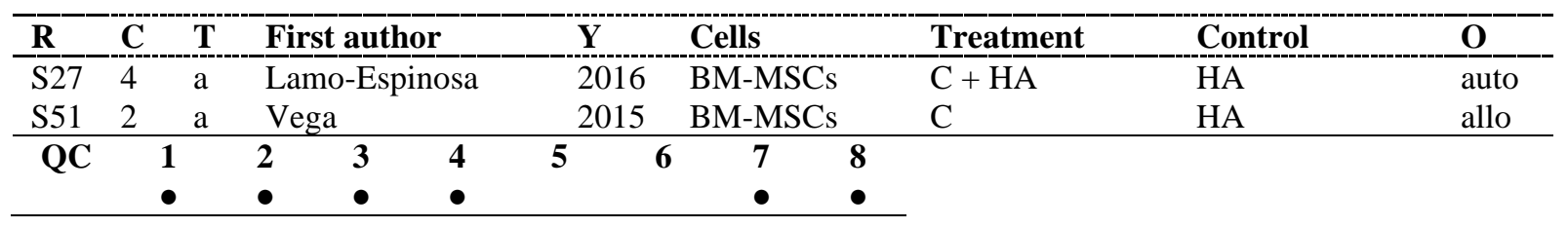


Table S8 | Details of the sub-analyses performed in a meta-analysis by Qu and $\mathrm{Sun}^{30}$.

Abbreviations: allo $=$ allogeneic cells; auto $=$ autologous cells; $\mathrm{C}=$ category of study as outlined in Table S2; $\mathrm{O}=$ origin of cells; $\mathrm{QC}=$ quality criteria (outlined in Table 1 in the main text; a point indicates that the corresponding quality criterion was fulfilled); $\mathrm{R}=$ reference number; $\mathrm{T}=$ type of study as outlined in Table S3; Y = year of publication. The abbreviations of the cell types and treatments are provided in Table S6.

VAS Pain score reported at 3 months post treatment

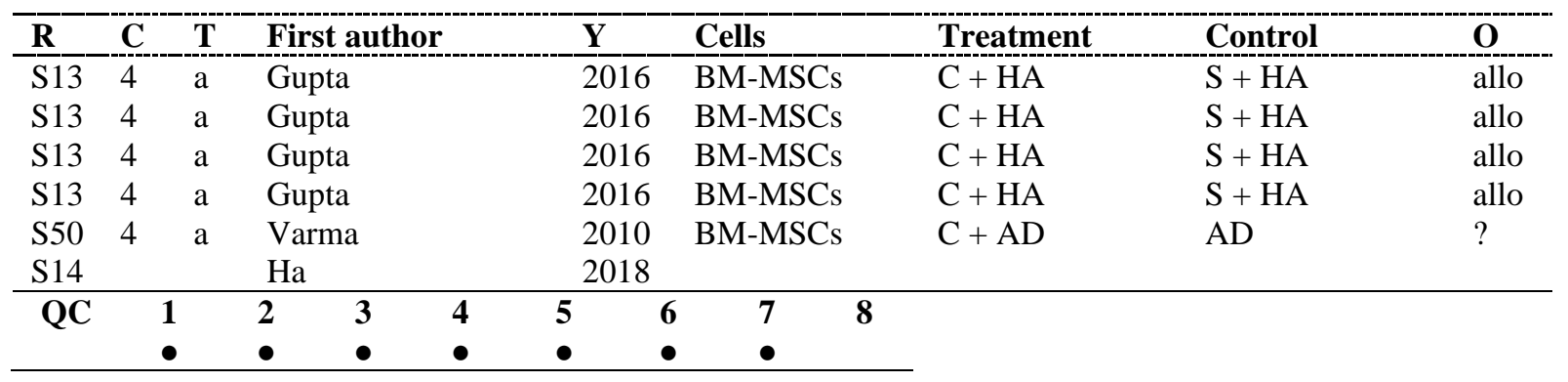

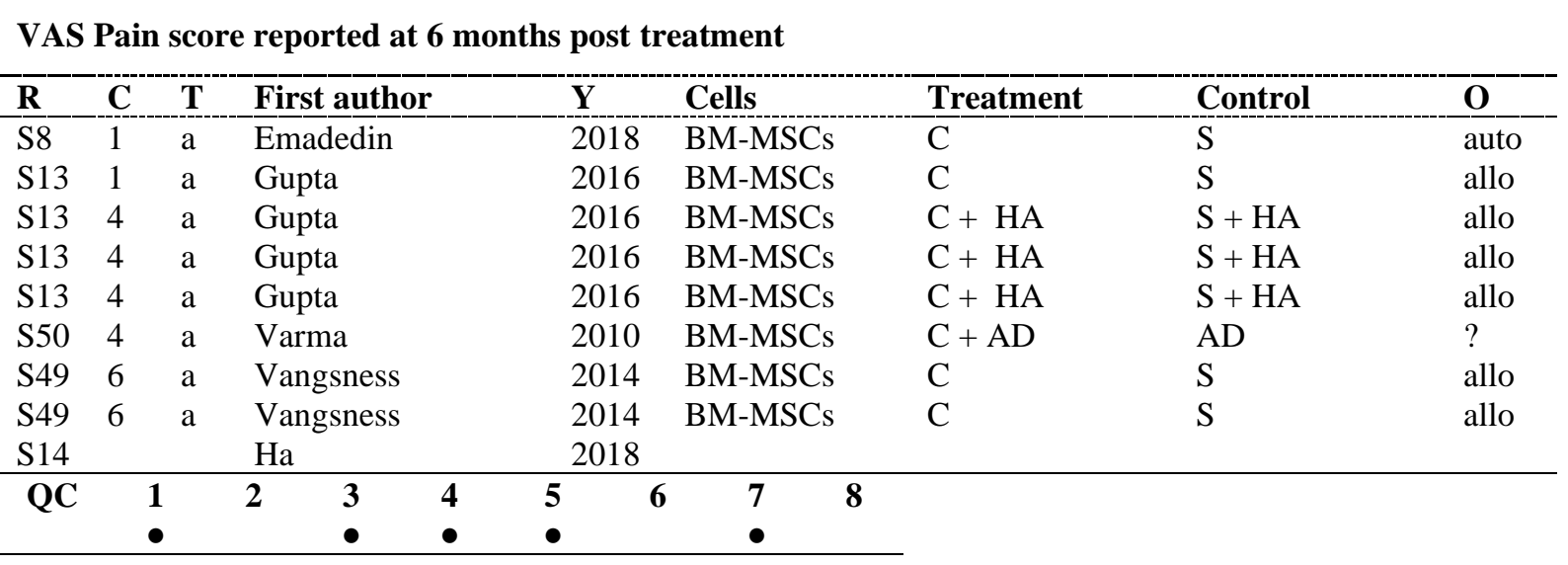

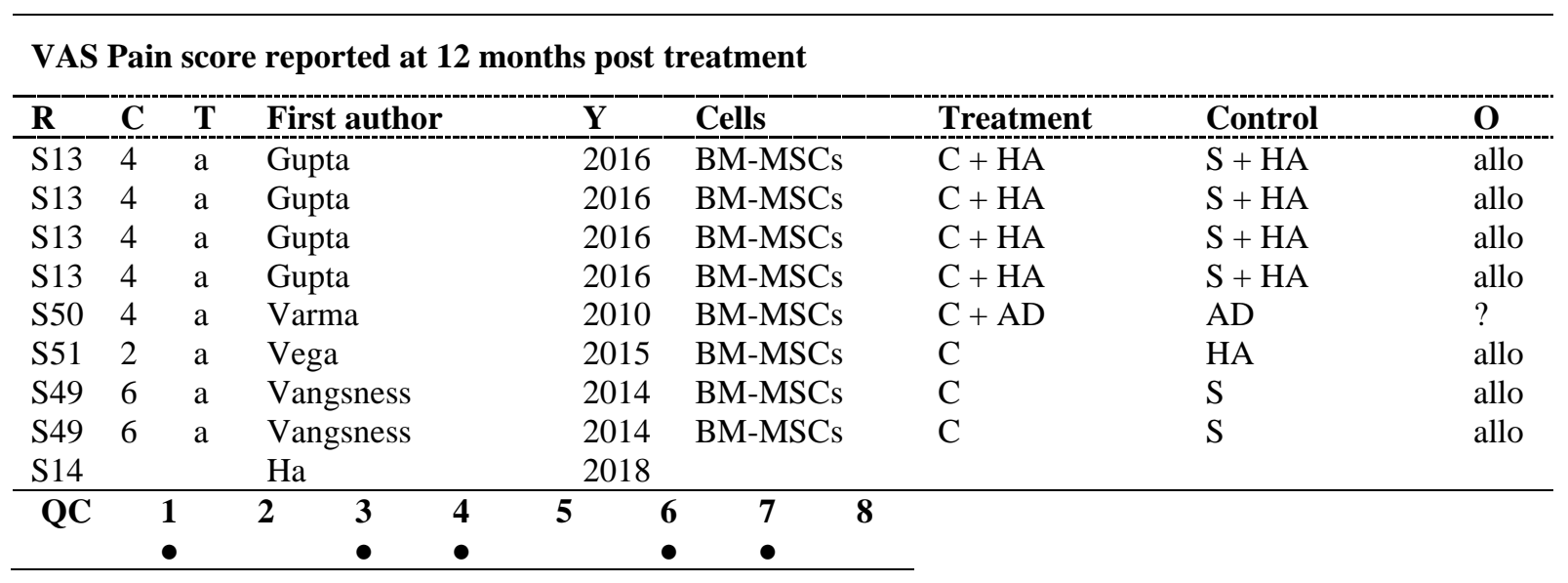


VAS Pain score reported at 24 months post treatment

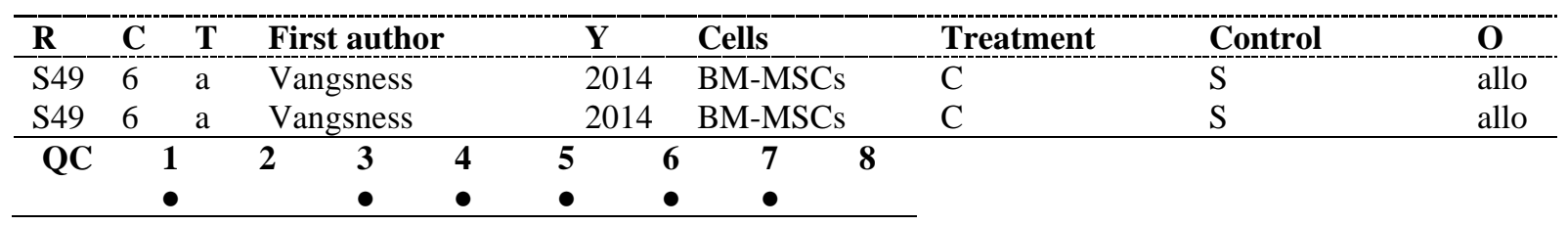

WOMAC Pain score reported at 3 months post treatment

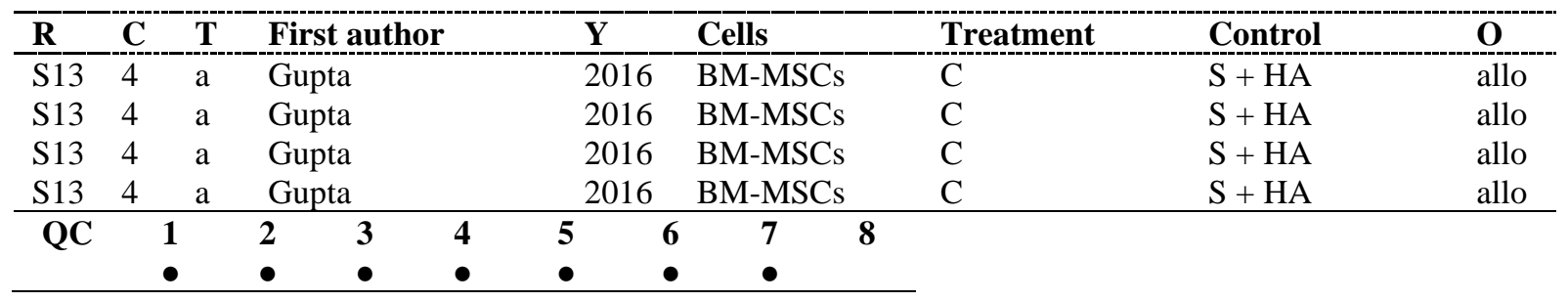

WOMAC Pain score reported at 6 months post treatment

\begin{tabular}{|c|c|c|c|c|c|c|c|c|}
\hline $\mathbf{R}$ & $\mathbf{C}$ & $\mathbf{T}$ & First author & $\mathbf{Y}$ & Cells & Treatment & Control & $\mathbf{O}$ \\
\hline S8 & 1 & $a$ & Emadedin & 2018 & BM-MSCs & $\mathrm{C}$ & $\mathrm{S}$ & auto \\
\hline S27 & 4 & $\mathrm{a}$ & Lamo-Espinosa & 2016 & BM-MSCs & $\mathrm{C}+\mathrm{HA}$ & HA & auto \\
\hline S27 & 4 & $\mathrm{a}$ & Lamo-Espinosa & 2016 & BM-MSCs & $\mathrm{C}+\mathrm{HA}$ & HA & auto \\
\hline S13 & 4 & $\mathrm{a}$ & Gupta & 2016 & BM-MSCs & $\mathrm{C}+\mathrm{HA}$ & $\mathrm{S}+\mathrm{HA}$ & allo \\
\hline S13 & 4 & $\mathrm{a}$ & Gupta & 2016 & BM-MSCs & $\mathrm{C}+\mathrm{HA}$ & $\mathrm{S}+\mathrm{HA}$ & allo \\
\hline S13 & 4 & $\mathrm{a}$ & Gupta & 2016 & BM-MSCs & $\mathrm{C}+\mathrm{HA}$ & $\mathrm{S}+\mathrm{HA}$ & allo \\
\hline $\mathrm{S} 13$ & 4 & $\mathrm{a}$ & Gupta & 2016 & BM-MSCs & $\mathrm{C}+\mathrm{HA}$ & $\mathrm{S}+\mathrm{HA}$ & allo \\
\hline \multirow[t]{2}{*}{$\mathbf{Q C}$} & & & 23 & $5 \quad 6$ & 7 & & & \\
\hline & & & $\bullet$ & $\bullet$ & $\bullet$ & & & \\
\hline
\end{tabular}

WOMAC Pain score reported at 12 months post treatment

\begin{tabular}{|c|c|c|c|c|c|c|c|c|}
\hline $\mathbf{R}$ & $\mathbf{C}$ & $\mathbf{T}$ & First author & $\mathbf{Y}$ & Cells & Treatment & Control & $\mathbf{O}$ \\
\hline $\mathrm{S} 27$ & 4 & $\mathrm{a}$ & Lamo-Espinosa & 2016 & BM-MSCs & $\mathrm{C}+\mathrm{HA}$ & $\mathrm{HA}$ & auto \\
\hline S27 & 4 & $\mathrm{a}$ & Lamo-Espinosa & 2016 & BM-MSCs & $\mathrm{C}+\mathrm{HA}$ & $\mathrm{HA}$ & auto \\
\hline $\mathrm{S} 13$ & 4 & $\mathrm{a}$ & Gupta & 2016 & BM-MSCs & $\mathrm{C}+\mathrm{HA}$ & $\mathrm{S}+\mathrm{HA}$ & allo \\
\hline S13 & 4 & $\mathrm{a}$ & Gupta & 2016 & BM-MSCs & $\mathrm{C}+\mathrm{HA}$ & $\mathrm{S}+\mathrm{HA}$ & allo \\
\hline S13 & 4 & $\mathrm{a}$ & Gupta & 2016 & BM-MSCs & $\mathrm{C}+\mathrm{HA}$ & $\mathrm{S}+\mathrm{HA}$ & allo \\
\hline $\mathrm{S} 13$ & 4 & $\mathrm{a}$ & Gupta & 2016 & BM-MSCs & $\mathrm{C}+\mathrm{HA}$ & $\mathrm{S}+\mathrm{HA}$ & allo \\
\hline S51 & 2 & $\mathrm{a}$ & Vega & 2015 & BM-MSCs & $\mathrm{C}+\mathrm{HA}$ & HA & allo \\
\hline \multirow[t]{2}{*}{$\mathbf{Q C}$} & & & $\begin{array}{ll}2 & 3\end{array}$ & $5 \quad 6$ & $\begin{array}{ll}7 & 8\end{array}$ & & & \\
\hline & & & $\bullet$ & & $\bullet$ & & & \\
\hline
\end{tabular}


Table S9 | Details of the sub-analyses performed in a meta-analysis by Ma et al. ${ }^{18}$.

Abbreviations: allo = allogeneic cells; auto = autologous cells; $\mathrm{C}=$ category of study as outlined in Table S2; $\mathrm{O}=$ origin of cells; $\mathrm{QC}=$ quality criteria (outlined in Table 1 in the main text; a point indicates that the corresponding quality criterion was fulfilled); $\mathrm{R}=$ reference number; $\mathrm{T}=$ type of study as outlined in Table S3; Y = year of publication. The abbreviations of the cell types and treatments are provided in Table S6.

\begin{tabular}{|c|c|c|c|c|c|c|c|c|}
\hline \multicolumn{9}{|c|}{ VAS Pain score } \\
\hline $\mathbf{R}$ & C & $\mathbf{T}$ & First author & $\mathbf{Y}$ & Cells & Treatment & Control & O \\
\hline $\mathrm{S} 8$ & 1 & $\mathrm{a}$ & Emadedin & 2018 & BM-MSCs & $\mathrm{C}$ & $\mathrm{S}$ & auto \\
\hline S13 & 4 & $\mathrm{a}$ & Gupta & 2016 & BM-MSCs & $\mathrm{C}+\mathrm{HA}$ & $\mathrm{S}+\mathrm{HA}$ & allo \\
\hline S26 & 1 & $\mathrm{a}$ & Kuah & 2018 & ADSCs & $\mathrm{C}$ & $\mathrm{S}$ & allo \\
\hline S27 & 4 & $\mathrm{a}$ & Lamo-Espinosa & 2016 & BM-MSCs & $\mathrm{C}+\mathrm{HA}$ & HA & auto \\
\hline S29 & 2 & a & $\mathrm{Lu}$ & 2019 & ADSCs & $\mathrm{C}$ & HA & auto \\
\hline S31 & 2 & $\mathrm{a}$ & Matas & 2019 & hUC-MSCs & $\mathrm{C}$ & HA & allo \\
\hline S51 & 2 & $\mathrm{a}$ & Vega & 2015 & BM-MSCs & $\mathrm{C}$ & HA & allo \\
\hline QC & & & $\begin{array}{ll}2 & 3 \\
\bullet & \bullet\end{array}$ & $5 \quad 6$ & $\begin{array}{ll}7 & 8 \\
\bullet & \bullet\end{array}$ & & & \\
\hline
\end{tabular}

\begin{tabular}{|c|c|c|c|c|c|c|c|c|}
\hline $\mathbf{R}$ & C & $T$ & First author & $\mathbf{Y}$ & Cells & Treatment & Control & 0 \\
\hline S8 & 1 & $\mathrm{a}$ & Emadedin & 2018 & BM-MSCs & $\mathrm{C}$ & $\mathrm{S}$ & auto \\
\hline S9 & 3 & $\mathrm{a}$ & Freitag & 2019 & ADSCs & $\mathrm{C}$ & $\mathrm{CM}$ & auto \\
\hline S13 & 4 & $\mathrm{a}$ & Gupta & 2016 & BM-MSCs & $\mathrm{C}+\mathrm{HA}$ & $\mathrm{S}+\mathrm{HA}$ & allo \\
\hline S27 & 4 & $\mathrm{a}$ & Lamo-Espinosa & 2016 & BM-MSCs & $\mathrm{C}+\mathrm{HA}$ & HA & auto \\
\hline S29 & 2 & $\mathrm{a}$ & $\mathrm{Lu}$ & 2019 & ADSCs & $\mathrm{C}$ & HA & auto \\
\hline S31 & 2 & $\mathrm{a}$ & Matas & 2019 & hUC-MSCs & $\mathrm{C}$ & HA & allo \\
\hline S51 & 2 & $\mathrm{a}$ & Vega & 2015 & BM-MSCs & $\mathrm{C}$ & $\mathrm{HA}$ & allo \\
\hline$\overline{Q C}$ & & & $\begin{array}{lll}2 & 3 & 4 \\
\bullet & \bullet & \bullet\end{array}$ & $\begin{array}{ll}5 & 6\end{array}$ & $\begin{array}{l}7 \\
\bullet\end{array}$ & & & \\
\hline
\end{tabular}

\section{WOMAC Pain score}

\begin{tabular}{|c|c|c|c|c|c|c|c|c|}
\hline $\mathbf{R}$ & C & $\mathbf{T}$ & First author & $\mathbf{Y}$ & Cells & Treatment & Control & O \\
\hline S8 & 1 & $\mathrm{a}$ & Emadedin & 2018 & BM-MSCs & $\mathrm{C}$ & $\mathrm{S}$ & auto \\
\hline S13 & 4 & $\mathrm{a}$ & Gupta & 2016 & BM-MSCs & $\mathrm{C}+\mathrm{HA}$ & $\mathrm{S}+\mathrm{HA}$ & allo \\
\hline S26 & 1 & $\mathrm{a}$ & Kuah & 2018 & ADSCs & $\mathrm{C}$ & $\mathrm{S}$ & allo \\
\hline S27 & 4 & $\mathrm{a}$ & Lamo-Espinosa & 2016 & BM-MSCs & $\mathrm{C}+\mathrm{HA}$ & HA & auto \\
\hline S29 & 2 & $\mathrm{a}$ & $\mathrm{Lu}$ & 2019 & ADSCs & $\mathrm{C}$ & HA & auto \\
\hline S31 & 2 & $\mathrm{a}$ & Matas & 2019 & hUC-MSCs & $\mathrm{C}$ & HA & allo \\
\hline S51 & 2 & $\mathrm{a}$ & Vega & 2015 & BM-MSCs & $\mathrm{C}$ & HA & allo \\
\hline QC & & & $\begin{array}{l}3 \\
\bullet\end{array}$ & 5 & $\begin{array}{l}7 \\
\bullet \\
\end{array}$ & & & \\
\hline
\end{tabular}




\section{WOMAC Stiffness score}

\begin{tabular}{|c|c|c|c|c|c|c|c|c|}
\hline $\mathbf{R}$ & $\mathbf{C}$ & $\mathbf{T}$ & First author & $\mathbf{Y}$ & Cells & Treatment & Control & $\mathbf{O}$ \\
\hline S8 & 1 & $\mathrm{a}$ & Emadedin & 2018 & BM-MSCs & $\mathrm{C}$ & $\mathrm{S}$ & auto \\
\hline S13 & 4 & $\mathrm{a}$ & Gupta & 2016 & BM-MSCs & $\mathrm{C}+\mathrm{HA}$ & $\mathrm{S}+\mathrm{HA}$ & allo \\
\hline S27 & 4 & $\mathrm{a}$ & Lamo-Espinosa & 2016 & BM-MSCs & $\mathrm{C}+\mathrm{HA}$ & HA & auto \\
\hline S29 & 2 & $\mathrm{a}$ & $\mathrm{Lu}$ & 2019 & $\mathrm{ADSCs}$ & $\mathrm{C}$ & HA & auto \\
\hline S31 & 2 & $\mathrm{a}$ & Matas & 2019 & hUC-MSCs & $\mathrm{C}$ & HA & allo \\
\hline \multirow[t]{2}{*}{$\mathbf{Q C}$} & & & 23 & $5 \quad 6$ & $\begin{array}{ll}7 & 8\end{array}$ & & & \\
\hline & & & $\bullet$ & & $\bullet$ & & & \\
\hline
\end{tabular}

\section{WOMAC Function score}

\begin{tabular}{|c|c|c|c|c|c|c|c|c|}
\hline $\mathbf{R}$ & $\mathrm{C}$ & $\mathbf{T}$ & First author & $\mathbf{Y}$ & Cells & Treatment & Control & $\mathbf{O}$ \\
\hline S8 & 1 & $\mathrm{a}$ & Emadedin & 2018 & BM-MSCs & $\mathrm{C}$ & $\mathrm{S}$ & auto \\
\hline S13 & 4 & $\mathrm{a}$ & Gupta & 2016 & BM-MSCs & $\mathrm{C}+\mathrm{HA}$ & $\mathrm{S}+\mathrm{HA}$ & allo \\
\hline S27 & 4 & $\mathrm{a}$ & Lamo-Espinosa & 2016 & BM-MSCs & $\mathrm{C}+\mathrm{HA}$ & HA & auto \\
\hline S29 & 2 & $\mathrm{a}$ & $\mathrm{Lu}$ & 2019 & ADSCs & $\mathrm{C}$ & HA & auto \\
\hline S31 & 2 & $\mathrm{a}$ & Matas & 2019 & hUC-MSCs & $\mathrm{C}$ & HA & allo \\
\hline QC & & & $\begin{array}{ll}2 & 3 \\
\bullet & \bullet \\
\end{array}$ & $5 \quad 6$ & $\begin{array}{ll}7 \\
\bullet\end{array}$ & & & \\
\hline
\end{tabular}

Whole-Organ Magnetic Resonance Imaging (WORMS) score

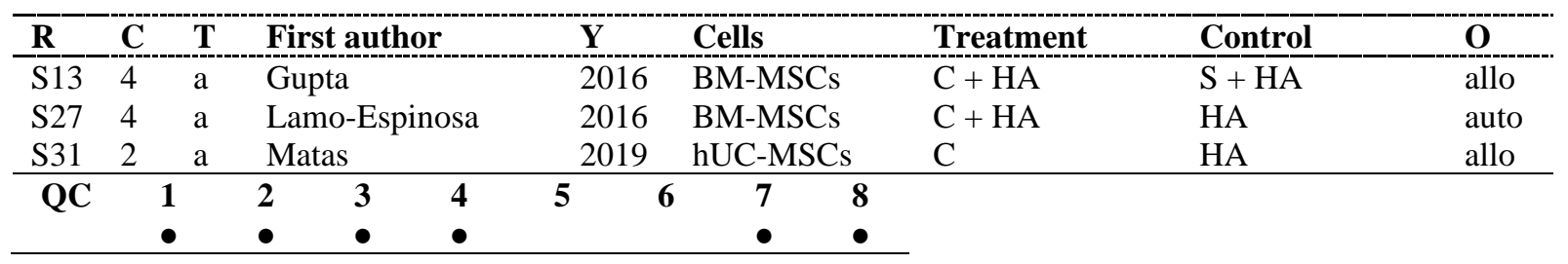

\section{Cartilage volume}

\begin{tabular}{|c|c|c|c|c|c|c|c|c|}
\hline $\mathbf{R}$ & $\mathbf{C}$ & $\mathbf{T}$ & First author & $\mathbf{Y}$ & Cells & Treatment & Control & O \\
\hline S26 & 1 & $\mathrm{a}$ & Kuah & 2018 & ADSCs & $\mathrm{C}$ & $\mathrm{S}$ & allo \\
\hline S28 & 1 & $\mathrm{a}$ & Lee & 2019 & ADSCs & $\mathrm{C}$ & S & auto \\
\hline S29 & 2 & $\mathrm{a}$ & $\mathrm{Lu}$ & 2019 & ADSCs & $\mathrm{C}$ & HA & auto \\
\hline
\end{tabular}

Number of patients with adverse events

\begin{tabular}{|c|c|c|c|c|c|c|c|c|}
\hline $\mathbf{R}$ & C & $\mathbf{T}$ & First author & $\mathbf{Y}$ & Cells & Treatment & Control & $\mathbf{0}$ \\
\hline S13 & 4 & $\mathrm{a}$ & Gupta & 2016 & BM-MSCs & $\mathrm{C}+\mathrm{HA}$ & $\mathrm{S}+\mathrm{HA}$ & allo \\
\hline $\mathrm{S} 21$ & 1 & $\mathrm{a}$ & Khalifeh Soltani & 2019 & P-MSCs & $\mathrm{C}$ & $\mathrm{S}$ & allo \\
\hline S26 & 1 & $\mathrm{a}$ & Kuah & 2018 & ADSCs & $\mathrm{C}$ & S & allo \\
\hline $\mathrm{S} 27$ & 4 & $\mathrm{a}$ & Lamo-Espinosa & 2016 & BM-MSCs & $\mathrm{C}+\mathrm{HA}$ & HA & auto \\
\hline $\mathrm{S} 28$ & 1 & $\mathrm{a}$ & Lee & 2019 & ADSCs & $\mathrm{C}$ & $\mathrm{S}$ & auto \\
\hline S29 & 2 & $\mathrm{a}$ & $\mathrm{Lu}$ & 2019 & ADSCs & $\mathrm{C}$ & $\mathrm{HA}$ & auto \\
\hline \multirow[t]{2}{*}{ QC } & & & 2 & $5 \quad 6$ & 7 & & & \\
\hline & & & $\bullet$ & & $\bullet$ & & & \\
\hline
\end{tabular}


Table S10 | Details of the sub-analyses performed in a meta-analysis by Huang et al. ${ }^{16}$.

Abbreviations: allo $=$ allogeneic cells; auto $=$ autologous cells; $\mathrm{C}=$ category of study as outlined in Table S2; $\mathrm{O}=$ origin of cells; $\mathrm{QC}=$ quality criteria (outlined in Table 1 in the main text; a point indicates that the corresponding quality criterion was fulfilled); $\mathrm{R}=$ reference number; $\mathrm{T}=$ type of study as outlined in Table S3; Y = year of publication. The abbreviations of the cell types and treatments are provided in Table S6.

VAS Pain score reported at 3 months post treatment

\begin{tabular}{|c|c|c|c|c|c|c|c|c|}
\hline $\mathbf{R}$ & $\mathbf{C}$ & $\mathbf{T}$ & First author & $\mathbf{Y}$ & Cells & Treatment & Control & $\mathbf{O}$ \\
\hline S6 & & & Bhattacharia & 2010 & & & & \\
\hline S26 & 1 & $\mathrm{a}$ & Kuah & 2018 & ADSCs & $\mathrm{C}$ & $S$ & allo \\
\hline S26 & 1 & $\mathrm{a}$ & Kuah & 2018 & ADSCs & $\mathrm{C}$ & $S$ & allo \\
\hline $\mathrm{S} 27$ & 4 & $\mathrm{a}$ & Lamo-Espinosa & 2016 & BM-MSCs & $\mathrm{C}+\mathrm{HA}$ & HA & auto \\
\hline S27 & 4 & $\mathrm{a}$ & Lamo-Espinosa & 2016 & BM-MSCs & $\mathrm{C}+\mathrm{HA}$ & HA & auto \\
\hline S41 & 4 & $\mathrm{~b}$ & Shapiro & 2017 & BMACBMAC & $\mathrm{C}+\mathrm{PPP}$ & $\mathrm{S}$ & auto \\
\hline QC & & & $\begin{array}{ll}2 & 3 \\
- & \\
\end{array}$ & $5 \quad 6$ & $\begin{array}{ll}7 & 8\end{array}$ & & & \\
\hline
\end{tabular}

\begin{tabular}{|c|c|c|c|c|c|c|c|c|}
\hline $\mathbf{R}$ & C & $T$ & First author & $\mathbf{Y}$ & Cells & Treatment & Control & O \\
\hline S6 & & & Bhattacharia & 2010 & & & & \\
\hline S26 & 1 & $\mathrm{a}$ & Kuah & 2018 & ADSCs & $\mathrm{C}$ & $\mathrm{S}$ & allo \\
\hline S26 & 1 & $\mathrm{a}$ & Kuah & 2018 & ADSCs & $\mathrm{C}$ & $\mathrm{S}$ & allo \\
\hline S27 & 4 & $\mathrm{a}$ & Lamo-Espinosa & 2016 & BM-MSCs & $\mathrm{C}+\mathrm{HA}$ & HA & auto \\
\hline S27 & 4 & $\mathrm{a}$ & Lamo-Espinosa & 2016 & BM-MSCs & $\mathrm{C}+\mathrm{HA}$ & HA & auto \\
\hline $\mathrm{S} 41$ & 4 & $\mathrm{~b}$ & Shapiro & 2017 & BMACBMAC & $\mathrm{C}+\mathrm{PPP}$ & S & auto \\
\hline QC & & 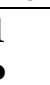 & $\begin{array}{ll}2 & 3 \\
\end{array}$ & $5 \quad 6$ & $\begin{array}{ll}7 & 8\end{array}$ & & & \\
\hline
\end{tabular}

\section{VAS pain score reported at 12 months post treatment}

\begin{tabular}{|c|c|c|c|c|c|c|c|c|}
\hline $\mathbf{R}$ & C & $\mathbf{T}$ & First author & $\mathbf{Y}$ & Cells & Treatment & Control & $\mathbf{O}$ \\
\hline S26 & 1 & $\mathrm{a}$ & Kuah & 2018 & ADSCs & $\mathrm{C}$ & $\mathrm{S}$ & allo \\
\hline S26 & 1 & $\mathrm{a}$ & Kuah & 2018 & ADSCs & $\mathrm{C}$ & S & allo \\
\hline S27 & 4 & $\mathrm{a}$ & Lamo-Espinosa & 2016 & BM-MSCs & $\mathrm{C}+\mathrm{HA}$ & HA & auto \\
\hline $\mathrm{S} 27$ & 4 & $\mathrm{a}$ & Lamo-Espinosa & 2016 & BM-MSCs & $\mathrm{C}+\mathrm{HA}$ & HA & auto \\
\hline S41 & 4 & $b$ & Shapiro & 2017 & BMACBMAC & $\mathrm{C}+\mathrm{PPP}$ & S & auto \\
\hline S51 & 2 & $\mathrm{a}$ & Vega & 2015 & BM-MSCs & $\mathrm{C}$ & HA & allo \\
\hline \multirow[t]{2}{*}{ QC } & & & 23 & $5 \quad 6$ & $\begin{array}{ll}7 & 8\end{array}$ & & & \\
\hline & & & $\bullet$ & & & & & \\
\hline
\end{tabular}


WOMAC Pain score reported at 3 months post treatment

\begin{tabular}{|c|c|c|c|c|c|c|c|c|}
\hline $\mathbf{R}$ & $\mathbf{C}$ & $\mathbf{T}$ & First author & $\mathbf{Y}$ & Cells & Treatment & Control & $\mathbf{0}$ \\
\hline S26 & 1 & $\mathrm{a}$ & Kuah & 2018 & ADSCs & $\mathrm{C}$ & $\mathrm{S}$ & allo \\
\hline S26 & 1 & $\mathrm{a}$ & Kuah & 2018 & ADSCs & $\mathrm{C}$ & S & allo \\
\hline S27 & 4 & $\mathrm{a}$ & Lamo-Espinosa & 2016 & BM-MSCs & $\mathrm{C}+\mathrm{HA}$ & HA & auto \\
\hline S27 & 4 & $\mathrm{a}$ & Lamo-Espinosa & 2016 & BM-MSCs & $\mathrm{C}+\mathrm{HA}$ & HA & auto \\
\hline QC & & & $\begin{array}{lll}2 & 3 & 4\end{array}$ & $5 \quad 6$ & $\begin{array}{ll}7 & 8\end{array}$ & & & \\
\hline & & & - & - & $\bullet$ & & & \\
\hline
\end{tabular}

WOMAC Pain score reported at 6 months post treatment

\begin{tabular}{|c|c|c|c|c|c|c|c|c|}
\hline $\mathbf{R}$ & $\mathbf{C}$ & $\mathbf{T}$ & First author & $\mathbf{Y}$ & Cells & Treatment & Control & $\mathbf{O}$ \\
\hline $\mathrm{S} 26$ & 1 & $\mathrm{a}$ & Kuah & 2018 & ADSCs & $\mathrm{C}$ & $\mathrm{S}$ & allo \\
\hline S26 & 1 & $\mathrm{a}$ & Kuah & 2018 & ADSCs & $\mathrm{C}$ & $\mathrm{S}$ & allo \\
\hline S27 & 4 & $\mathrm{a}$ & Lamo-Espinosa & 2016 & BM-MSCs & $\mathrm{C}+\mathrm{HA}$ & HA & auto \\
\hline S27 & 4 & $\mathrm{a}$ & Lamo-Espinosa & 2016 & BM-MSCs & $\mathrm{C}+\mathrm{HA}$ & $\mathrm{HA}$ & auto \\
\hline $\mathbf{Q C}$ & & & $\begin{array}{lll}2 & 3 & 4\end{array}$ & $5 \quad 6$ & $\begin{array}{ll}7 & 8\end{array}$ & & & \\
\hline
\end{tabular}

\section{WOMAC Pain score reported at 12 months post treatment}

\begin{tabular}{|c|c|c|c|c|c|c|c|c|}
\hline $\mathbf{R}$ & $\mathbf{C}$ & $\mathbf{T}$ & First author & $\mathbf{Y}$ & Cells & Treatment & Control & $\mathbf{O}$ \\
\hline S26 & 1 & $\mathrm{a}$ & Kuah & 2018 & ADSCs & $\mathrm{C}$ & $\mathrm{S}$ & allo \\
\hline S27 & 4 & $\mathrm{a}$ & Lamo-Espinosa & 2016 & BM-MSCs & $\mathrm{C}+\mathrm{HA}$ & HA & auto \\
\hline S27 & 4 & $\mathrm{a}$ & Lamo-Espinosa & 2016 & BM-MSCs & $\mathrm{C}+\mathrm{HA}$ & HA & auto \\
\hline S51 & 2 & $\mathrm{a}$ & Vega & 2015 & BM-MSCs & $\mathrm{C}$ & HA & allo \\
\hline $\mathbf{Q C}$ & & & $\begin{array}{ll}2 & 3 \\
\bullet & \bullet \\
\end{array}$ & $\begin{array}{ll}5 & 6 \\
\bullet & \\
\end{array}$ & $\begin{array}{ll}7 & 8 \\
\bullet & \end{array}$ & & & \\
\hline
\end{tabular}

\section{WOMAC Stiffness score reported at 3 months post treatment}

\begin{tabular}{|c|c|c|c|c|c|c|c|c|}
\hline $\mathbf{R}$ & C & $\mathbf{T}$ & First author & $\mathbf{Y}$ & Cells & Treatment & Control & O \\
\hline S26 & 1 & $\mathrm{a}$ & Kuah & 2018 & ADSCs & $\mathrm{C}$ & $\mathrm{S}$ & allo \\
\hline S26 & 1 & $\mathrm{a}$ & Kuah & 2018 & ADSCs & $\mathrm{C}$ & S & allo \\
\hline S27 & 4 & $\mathrm{a}$ & Lamo-Espinosa & 2016 & BM-MSCs & $\mathrm{C}+\mathrm{HA}$ & HA & auto \\
\hline S27 & 4 & $\mathrm{a}$ & Lamo-Espinosa & 2016 & BM-MSCs & $\mathrm{C}+\mathrm{HA}$ & HA & auto \\
\hline QC & & & $\begin{array}{lll}2 & 3 & 4 \\
\bullet & \bullet & \bullet\end{array}$ & $\begin{array}{ll}5 & 6 \\
0 & \end{array}$ & $\begin{array}{l}7 \\
\bullet\end{array}$ & & & \\
\hline
\end{tabular}

WOMAC Stiffnes score reported at 6 months post treatment

\begin{tabular}{|c|c|c|c|c|c|c|c|c|}
\hline $\mathbf{R}$ & C & $\mathbf{T}$ & First author & $\mathbf{Y}$ & Cells & Treatment & Control & $\mathbf{O}$ \\
\hline $\mathrm{S} 26$ & 1 & $\mathrm{a}$ & Kuah & 2018 & $\mathrm{ADSCs}$ & $\mathrm{C}$ & $\mathrm{S}$ & allo \\
\hline S26 & 1 & $\mathrm{a}$ & Kuah & 2018 & ADSCs & $\mathrm{C}$ & $\mathrm{S}$ & allo \\
\hline S27 & 4 & $\mathrm{a}$ & Lamo-Espinosa & 2016 & BM-MSCs & $\mathrm{C}+\mathrm{HA}$ & HA & auto \\
\hline S27 & 4 & $\mathrm{a}$ & Lamo-Espinosa & 2016 & BM-MSCs & $\mathrm{C}+\mathrm{HA}$ & HA & auto \\
\hline $\mathbf{Q C}$ & & & $\begin{array}{lll}2 & 3 & 4 \\
\bullet & \bullet & \bullet \\
\end{array}$ & $\begin{array}{ll}5 & 6 \\
\bullet & \\
\end{array}$ & $\begin{array}{ll}7 & 8 \\
\bullet & \\
\end{array}$ & & & \\
\hline
\end{tabular}


WOMAC Stiffness score reported at 12 months post treatment

\begin{tabular}{|c|c|c|c|c|c|c|c|c|}
\hline $\mathbf{R}$ & $\mathbf{C}$ & $\mathbf{T}$ & First author & $\mathbf{Y}$ & Cells & Treatment & Control & $\mathbf{O}$ \\
\hline S26 & 1 & $\mathrm{a}$ & Kuah & 2018 & ADSCs & $\mathrm{C}$ & $\mathrm{S}$ & allo \\
\hline S26 & 1 & $\mathrm{a}$ & Kuah & 2018 & ADSCs & $\mathrm{C}$ & S & allo \\
\hline S27 & 4 & $\mathrm{a}$ & Lamo-Espinosa & 2016 & BM-MSCs & $\mathrm{C}+\mathrm{HA}$ & HA & auto \\
\hline S27 & 4 & $\mathrm{a}$ & Lamo-Espinosa & 2016 & BM-MSCs & $\mathrm{C}+\mathrm{HA}$ & HA & auto \\
\hline QC & & & $\begin{array}{lll}2 & 3 & 4 \\
\bullet & \bullet & \bullet \\
\end{array}$ & $\begin{array}{ll}5 & 6 \\
\bullet & \\
\end{array}$ & $\begin{array}{ll}7 & 8 \\
\bullet & \\
\end{array}$ & & & \\
\hline
\end{tabular}

WOMAC Function score reported at 3 months post treatment

\begin{tabular}{|c|c|c|c|c|c|c|c|c|}
\hline $\mathbf{R}$ & $\mathbf{C}$ & $\mathbf{T}$ & First author & $\mathbf{Y}$ & Cells & Treatment & Control & $\mathbf{O}$ \\
\hline $\mathrm{S} 26$ & 1 & $\mathrm{a}$ & Kuah & 2018 & ADSCs & $\mathrm{C}$ & $\mathrm{S}$ & allo \\
\hline S26 & 1 & $\mathrm{a}$ & Kuah & 2018 & ADSCs & $\mathrm{C}$ & $\mathrm{S}$ & allo \\
\hline S27 & 4 & $\mathrm{a}$ & Lamo-Espinosa & 2016 & BM-MSCs & $\mathrm{C}+\mathrm{HA}$ & HA & auto \\
\hline $\mathrm{S} 27$ & 4 & $\mathrm{a}$ & Lamo-Espinosa & 2016 & BM-MSCs & $\mathrm{C}+\mathrm{HA}$ & $\mathrm{HA}$ & auto \\
\hline $\mathbf{Q C}$ & & & $\begin{array}{|lll|}2 & 3 & 4\end{array}$ & $5 \quad 6$ & $\begin{array}{ll}7 & 8\end{array}$ & & & \\
\hline
\end{tabular}

\section{WOMAC Function score reported at 6 months post treatment}

\begin{tabular}{|c|c|c|c|c|c|c|c|c|}
\hline $\mathbf{R}$ & $\mathbf{C}$ & $\mathbf{T}$ & First author & $\mathbf{Y}$ & Cells & Treatment & Control & $\mathbf{O}$ \\
\hline S26 & 1 & $\mathrm{a}$ & Kuah & 2018 & ADSCs & $\mathrm{C}$ & $\mathrm{S}$ & allo \\
\hline S26 & 1 & $\mathrm{a}$ & Kuah & 2018 & ADSCs & $\mathrm{C}$ & S & allo \\
\hline S27 & 4 & $\mathrm{a}$ & Lamo-Espinosa & 2016 & BM-MSCs & $\mathrm{C}+\mathrm{HA}$ & HA & auto \\
\hline $\mathrm{S} 27$ & 4 & $\mathrm{a}$ & Lamo-Espinosa & 2016 & BM-MSCs & $\mathrm{C}+\mathrm{HA}$ & HA & auto \\
\hline QC & & & $\begin{array}{lll}2 & 3 & 4 \\
\bullet & \bullet & \bullet \\
\end{array}$ & $\begin{array}{ll}5 & 6 \\
\bullet & \\
\end{array}$ & $\begin{array}{ll}7 & 8 \\
\bullet & \\
\end{array}$ & & & \\
\hline
\end{tabular}

\section{WOMAC Function score reported at 12 months post treatment}

\begin{tabular}{|c|c|c|c|c|c|c|c|c|}
\hline $\mathbf{R}$ & $\mathbf{C}$ & $\mathbf{T}$ & First author & $\mathbf{Y}$ & Cells & Treatment & Control & O \\
\hline $\mathrm{S} 26$ & 1 & $\mathrm{a}$ & Kuah & 2018 & ADSCs & $\mathrm{C}$ & $\mathrm{S}$ & allo \\
\hline S26 & 1 & $\mathrm{a}$ & Kuah & 2018 & ADSCs & $\mathrm{C}$ & $\mathrm{S}$ & allo \\
\hline $\mathrm{S} 27$ & 4 & $\mathrm{a}$ & Lamo-Espinosa & 2016 & BM-MSCs & $\mathrm{C}+\mathrm{HA}$ & HA & auto \\
\hline S27 & 4 & $\mathrm{a}$ & Lamo-Espinosa & 2016 & BM-MSCs & $\mathrm{C}+\mathrm{HA}$ & HA & auto \\
\hline QC & & & $\begin{array}{lll}2 & 3 & 4 \\
\bullet & \bullet & \bullet \\
\end{array}$ & $\begin{array}{ll}5 & 6 \\
- & \\
\end{array}$ & $\begin{array}{ll}7 & 8 \\
\bullet & \\
\end{array}$ & & & \\
\hline
\end{tabular}

International Knee Documentation Committee (IKDC) score reported at 6 months post treatment

\begin{tabular}{|c|c|c|c|c|c|c|c|c|}
\hline $\mathbf{R}$ & C & $\mathbf{T}$ & First author & $\mathbf{Y}$ & Cells & Treatment & Control & $\mathbf{O}$ \\
\hline S38 & 6 & $a$ & Saw & 2013 & pBSCs & $\mathrm{C}+\mathrm{MF}+\mathrm{HA}$ & $\mathrm{MF}+\mathrm{HA}$ & auto \\
\hline S53 & 4 & $\mathrm{a}$ & Wong & 2013 & BM-MSCs & $\begin{array}{l}\mathrm{C}+\mathrm{MF}+\mathrm{HTO} \\
+\mathrm{HA}\end{array}$ & $\begin{array}{l}\mathrm{MF}+\mathrm{HTO}+ \\
\mathrm{HA}\end{array}$ & auto \\
\hline
\end{tabular}

\begin{tabular}{lllllllll}
\hline QC & $\mathbf{1}$ & $\mathbf{2}$ & $\mathbf{3}$ & $\mathbf{4}$ & $\mathbf{5}$ & $\mathbf{6}$ & $\mathbf{7}$ & $\mathbf{8}$ \\
& $\bullet$ & & $\bullet$ & $\bullet$ & $\bullet$ & $\bullet$ & $\bullet$ & $\bullet$ \\
\hline
\end{tabular}


International Knee Documentation Committee (IKDC) score reported at 12 months post treatment

\begin{tabular}{|c|c|c|c|c|c|c|c|c|}
\hline $\mathbf{R}$ & C & $\mathbf{T}$ & First author & $\mathbf{Y}$ & Cells & Treatment & Control & $\mathbf{O}$ \\
\hline S38 & 6 & $\mathrm{a}$ & Saw & 2013 & pBSCs & $\mathrm{C}+\mathrm{MF}+\mathrm{HA}$ & $\mathrm{MF}+\mathrm{HA}$ & auto \\
\hline S53 & 4 & $\mathrm{a}$ & Wong & 2013 & BM-MSCs & $\begin{array}{l}\mathrm{C}+\mathrm{MF}+\mathrm{HTO} \\
+\mathrm{HA}\end{array}$ & $\begin{array}{l}\mathrm{MF}+\mathrm{HTO}+ \\
\mathrm{HA}\end{array}$ & auto \\
\hline
\end{tabular}

\begin{tabular}{lllllllll}
\hline QC & $\mathbf{1}$ & $\mathbf{2}$ & $\mathbf{3}$ & $\mathbf{4}$ & $\mathbf{5}$ & $\mathbf{6}$ & $\mathbf{7}$ & $\mathbf{8}$ \\
& $\bullet$ & & $\bullet$ & $\bullet$ & $\bullet$ & $\bullet$ & $\bullet$ & $\bullet$ \\
\hline
\end{tabular}

International Knee Documentation Committee (IKDC) score reported at 24 months post treatment

\begin{tabular}{|c|c|c|c|c|c|c|c|c|}
\hline $\mathbf{R}$ & C & $\mathbf{T}$ & First author & $\mathbf{Y}$ & Cells & Treatment & Control & $\mathbf{O}$ \\
\hline S38 & 6 & $\mathrm{a}$ & Saw & 2013 & pBSCs & $\mathrm{C}+\mathrm{MF}+\mathrm{HA}$ & $\mathrm{MF}+\mathrm{HA}$ & auto \\
\hline S53 & 4 & $\mathrm{a}$ & Wong & 2013 & BM-MSCs & $\begin{array}{l}\mathrm{C}+\mathrm{MF}+\mathrm{HTO} \\
+\mathrm{HA}\end{array}$ & $\begin{array}{l}\mathrm{MF}+\mathrm{HTO}+ \\
\mathrm{HA}\end{array}$ & auto \\
\hline
\end{tabular}

\begin{tabular}{ccccccccc}
\hline QC & $\mathbf{1}$ & $\mathbf{2}$ & $\mathbf{3}$ & $\mathbf{4}$ & $\mathbf{5}$ & $\mathbf{6}$ & $\mathbf{7}$ & $\mathbf{8}$ \\
& $\bullet$ & & $\bullet$ & $\bullet$ & $\bullet$ & $\bullet$ & $\bullet$ & $\bullet$ \\
\hline
\end{tabular}

\section{Adverse events}

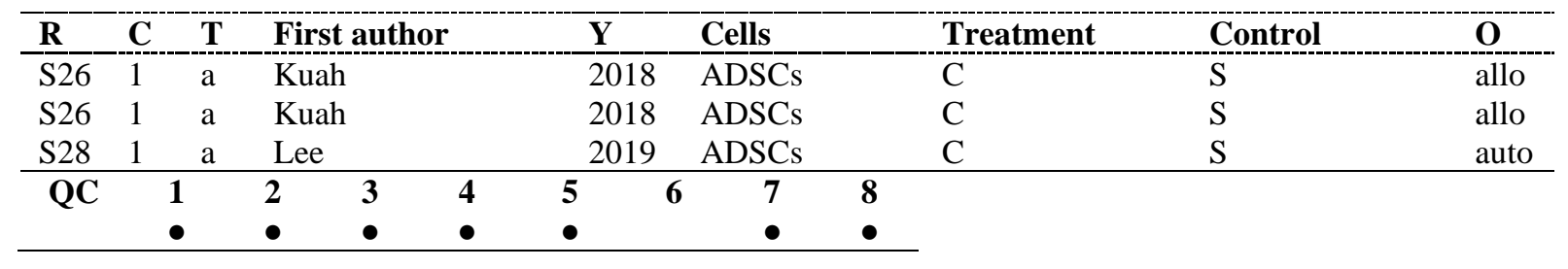


Table S11 | Details of the sub-analyses performed in a meta-analysis by Wei et al. ${ }^{32}$.

Abbreviations: allo $=$ allogeneic cells; auto $=$ autologous cells; $\mathrm{C}=$ category of study as outlined in Table S2; $\mathrm{O}=$ origin of cells; $\mathrm{QC}=$ quality criteria (outlined in Table 1 in the main text; a point indicates that the corresponding quality criterion was fulfilled); $\mathrm{R}=$ reference number; $\mathrm{T}=$ type of study as outlined in Table S3; Y = year of publication. The abbreviations of the cell types and treatments are provided in Table S6.

\begin{tabular}{|c|c|c|c|c|c|c|c|c|}
\hline \multicolumn{9}{|c|}{ VAS Pain score } \\
\hline $\mathbf{R}$ & C & $\mathbf{T}$ & First author & $\mathbf{Y}$ & Cells & Treatment & Control & 0 \\
\hline S51 & 2 & $\mathrm{a}$ & Vega & 2015 & BM-MSCs & $\mathrm{C}$ & HA & allo \\
\hline S13 & 4 & $\mathrm{a}$ & Gupta & 2016 & BM-MSCs & $\mathrm{C}+\mathrm{HA}$ & $\mathrm{S}+\mathrm{HA}$ & allo \\
\hline S27 & 4 & $\mathrm{a}$ & Lamo-Espinosa & 2016 & BM-MSCs & $\mathrm{C}+\mathrm{HA}$ & $\mathrm{HA}$ & auto \\
\hline S8 & 1 & $\mathrm{a}$ & Emadedin & 2018 & BM-MSCs & $\mathrm{C}$ & $\mathrm{S}$ & auto \\
\hline S26 & 1 & a & Kuah & 2018 & ADSCs & $\mathrm{C}$ & $\mathrm{S}$ & allo \\
\hline S31 & 2 & $\mathrm{a}$ & Matas & 2019 & hUC-MSCs & $\mathrm{C}$ & HA & allo \\
\hline S28 & 1 & $\mathrm{a}$ & Lee & 2019 & ADSCs & $\mathrm{C}$ & $\mathrm{S}$ & auto \\
\hline QC & & & 4 & $5 \quad 6$ & 7 & & & \\
\hline
\end{tabular}

\begin{tabular}{|c|c|c|c|c|c|c|c|c|}
\hline $\mathbf{R}$ & C & $\mathbf{T}$ & First author & $\mathbf{Y}$ & Cells & Treatment & Control & O \\
\hline S51 & 2 & $\mathrm{a}$ & Vega & 2015 & BM-MSCs & $\mathrm{C}$ & HA & allo \\
\hline S13 & 4 & $\mathrm{a}$ & Gupta & 2016 & BM-MSCs & $\mathrm{C}+\mathrm{HA}$ & $\mathrm{S}+\mathrm{HA}$ & allo \\
\hline S27 & 4 & $\mathrm{a}$ & Lamo-Espinosa & 2016 & BM-MSCs & $\mathrm{C}+\mathrm{HA}$ & HA & auto \\
\hline S8 & 1 & $\mathrm{a}$ & Emadedin & 2018 & BM-MSCs & $\mathrm{C}$ & $\mathrm{S}$ & auto \\
\hline S26 & 1 & $\mathrm{a}$ & Kuah & 2018 & ADSCs & $\mathrm{C}$ & $\mathrm{S}$ & allo \\
\hline S31 & 2 & $\mathrm{a}$ & Matas & 2019 & hUC-MSCs & $\mathrm{C}$ & HA & allo \\
\hline S28 & 1 & $\mathrm{a}$ & Lee & 2019 & ADSCs & $\mathrm{C}$ & $\mathrm{S}$ & auto \\
\hline $\mathbf{Q C}$ & & & 4 & $5 \quad 6$ & 7 & & & \\
\hline
\end{tabular}

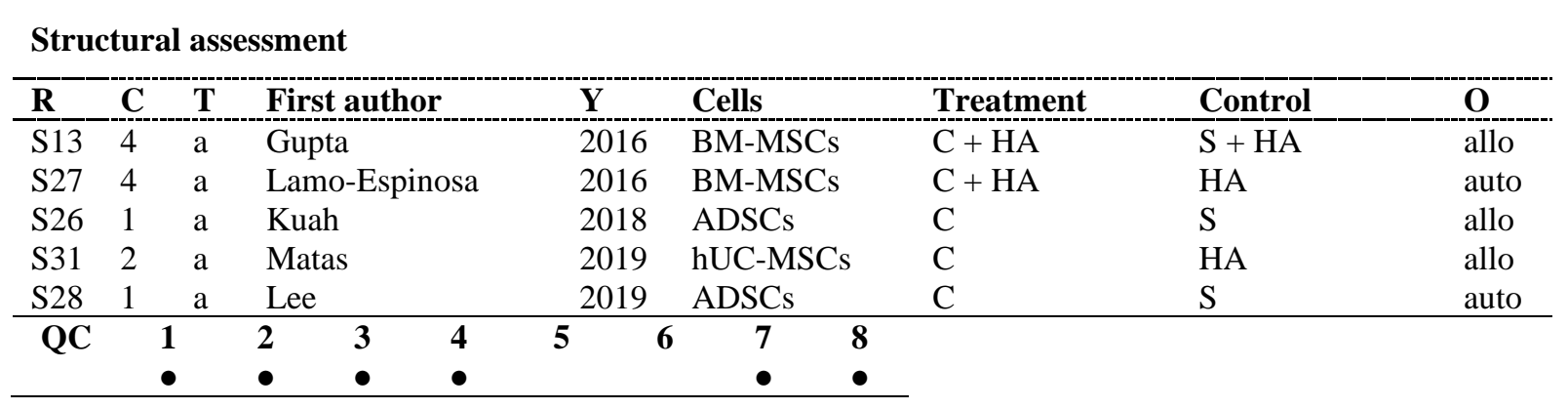


Table S12 | Details of the sub-analyses performed in a meta-analysis by Wang et al. ${ }^{20}$.

Abbreviations: allo $=$ allogeneic cells; auto $=$ autologous cells; $\mathrm{C}=$ category of study as outlined in Table S2; $\mathrm{O}=$ origin of cells; $\mathrm{QC}=$ quality criteria (outlined in Table 1 in the main text; a point indicates that the corresponding quality criterion was fulfilled); $\mathrm{R}=$ reference number; $\mathrm{T}=$ type of study as outlined in Table S3; Y = year of publication. The abbreviations of the cell types and treatments are provided in Table S6.

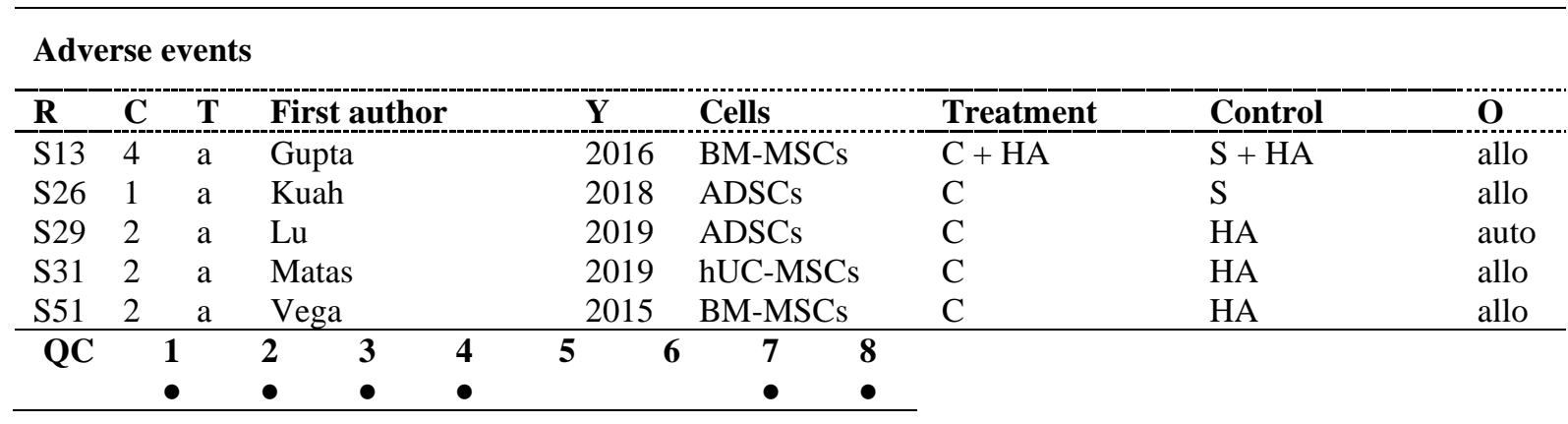

\begin{tabular}{|c|c|c|c|c|c|c|c|c|}
\hline $\mathbf{R}$ & C & $\mathbf{T}$ & First author & $\mathbf{Y}$ & Cells & Treatment & Control & O \\
\hline $\mathrm{S} 13$ & 4 & $\mathrm{a}$ & Gupta & 2016 & BM-MSCs & $\mathrm{C}+\mathrm{HA}$ & $\mathrm{S}+\mathrm{HA}$ & allo \\
\hline S26 & 1 & $\mathrm{a}$ & Kuah & 2018 & ADSCs & $\mathrm{C}$ & $\mathrm{S}$ & allo \\
\hline S27 & 4 & $\mathrm{a}$ & Lamo-Espinosa & 2016 & BM-MSCs & $\mathrm{C}+\mathrm{HA}$ & HA & auto \\
\hline S31 & 2 & $\mathrm{a}$ & Matas & 2019 & hUC-MSCs & $\mathrm{C}$ & HA & allo \\
\hline QC & & & $\begin{array}{ll}2 & 3 \\
\bullet & \bullet \\
\end{array}$ & $5 \quad 6$ & $\begin{array}{rr}7 & 8 \\
\bullet & \bullet \\
\end{array}$ & & & \\
\hline
\end{tabular}

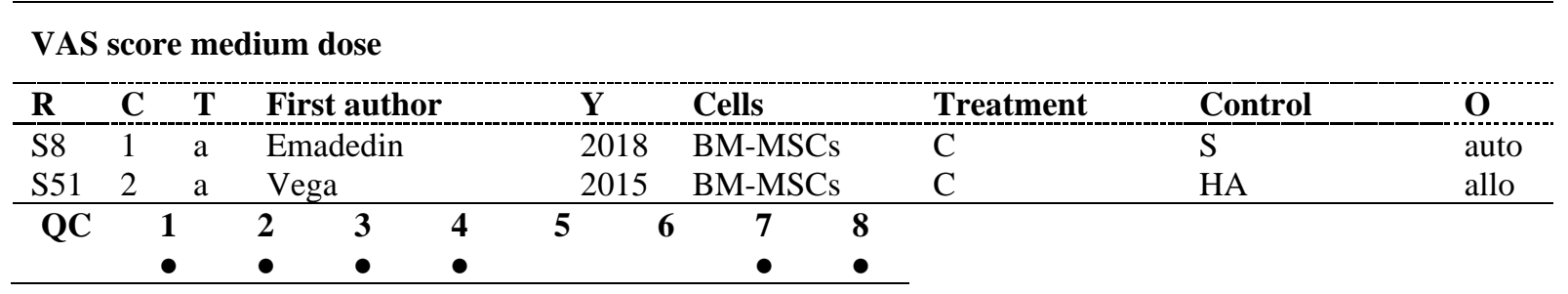

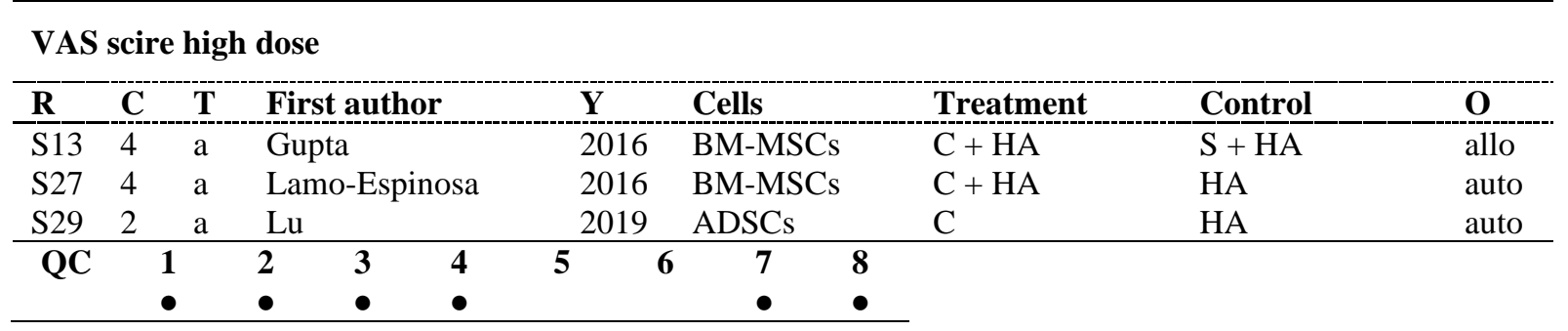


WOMAC Pain score low dose

\begin{tabular}{|c|c|c|c|c|c|c|c|c|}
\hline $\mathbf{R}$ & $\mathbf{C}$ & $\mathbf{T}$ & First author & $\mathbf{Y}$ & Cells & Treatment & Control & $\mathbf{O}$ \\
\hline S13 & 4 & $\mathrm{a}$ & Gupta & 2016 & BM-MSCs & $\mathrm{C}+\mathrm{HA}$ & $\mathrm{S}+\mathrm{HA}$ & allo \\
\hline S26 & 1 & $\mathrm{a}$ & Kuah & 2018 & ADSCs & $\mathrm{C}$ & $\mathrm{S}$ & allo \\
\hline S27 & 4 & $\mathrm{a}$ & Lamo-Espinosa & 2016 & BM-MSCs & $\mathrm{C}+\mathrm{HA}$ & HA & auto \\
\hline S31 & 2 & $\mathrm{a}$ & Matas & 2019 & hUC-MSCs & $\mathrm{C}$ & HA & allo \\
\hline QC & & & $\begin{array}{ll}2 & 3 \\
\bullet & \bullet \\
\end{array}$ & $5 \quad 6$ & $\begin{array}{ll}7 & 8 \\
\bullet & \\
\end{array}$ & & & \\
\hline
\end{tabular}

WOMAC Pain score medium dose

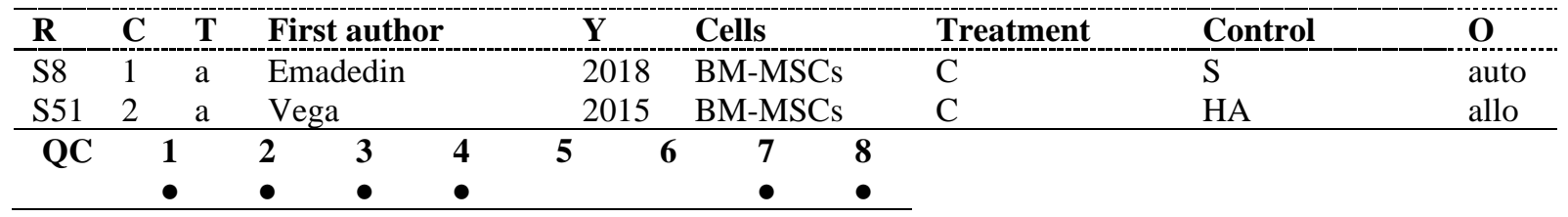

WOMAC Pain score high dose

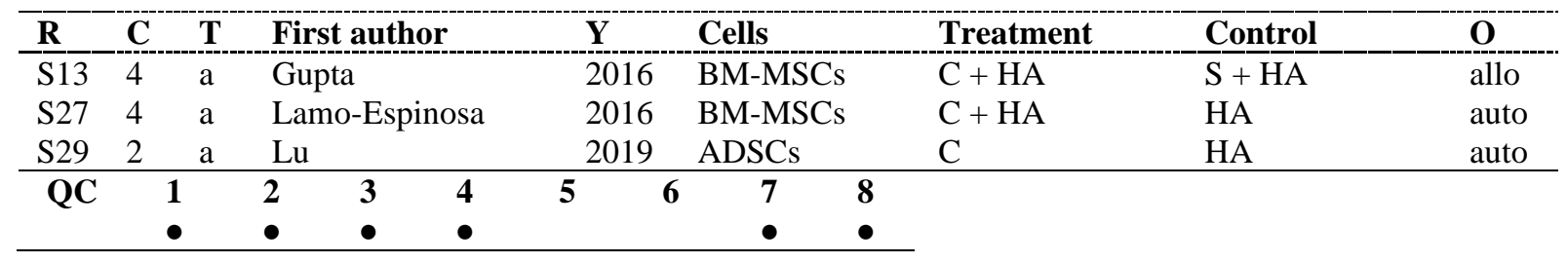

\section{WOMAC Stiffness score low dose}

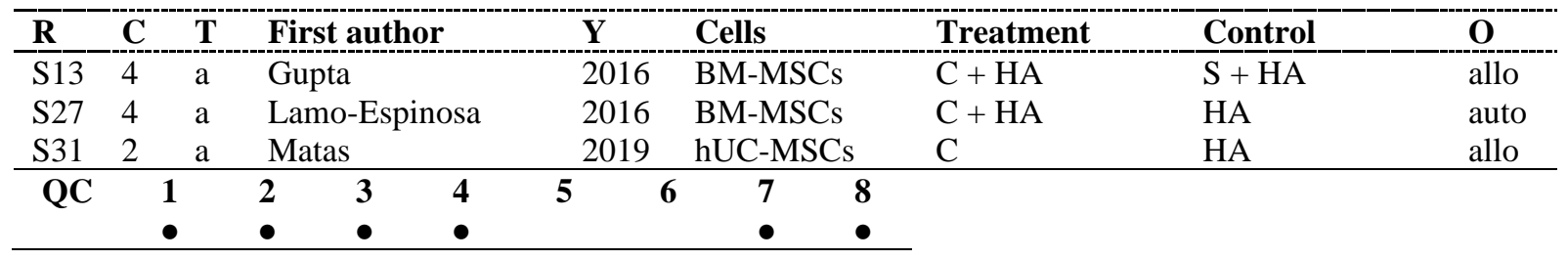

\section{WOMAC Stiffness score medium dose}

\begin{tabular}{|c|c|c|c|c|c|c|c|c|c|c|c|c|}
\hline $\mathbf{R}$ & C & $\mathbf{T}$ & $\mathbf{F}$ & au & & & & Cells & & Treatment & Control & $\mathbf{O}$ \\
\hline S8 & 1 & $\mathrm{a}$ & & edi & & & & BM-MSCs & & $\mathrm{C}$ & $\mathrm{S}$ & auto \\
\hline QC & & & $\begin{array}{l}2 \\
\bullet\end{array}$ & $\begin{array}{l}3 \\
\bullet\end{array}$ & $\begin{array}{l}4 \\
\bullet\end{array}$ & $\begin{array}{l}5 \\
\bullet\end{array}$ & $\begin{array}{l}6 \\
\bullet\end{array}$ & $\begin{array}{l}7 \\
\bullet\end{array}$ & • & & & \\
\hline
\end{tabular}


WOMAC Stiffness score high dose

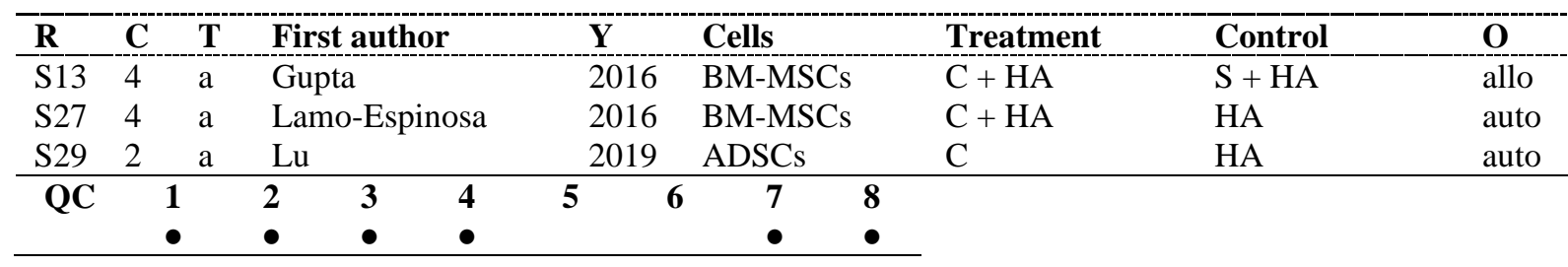

WOMAC Physical Function score low dose

\begin{tabular}{|c|c|c|c|c|c|c|c|c|}
\hline $\mathbf{R}$ & $\mathbf{C}$ & $\mathbf{T}$ & First author & $\mathbf{Y}$ & Cells & Treatment & Control & $\mathbf{O}$ \\
\hline $\mathrm{S} 13$ & 4 & $\mathrm{a}$ & Gupta & 2016 & BM-MSCs & $\mathrm{C}+\mathrm{HA}$ & $\mathrm{S}+\mathrm{HA}$ & allo \\
\hline S27 & 4 & $\mathrm{a}$ & Lamo-Espinosa & 2016 & BM-MSCs & $\mathrm{C}+\mathrm{HA}$ & HA & auto \\
\hline S31 & 2 & $\mathrm{a}$ & Matas & 2019 & hUC-MSCs & $\mathrm{C}$ & HA & allo \\
\hline $\mathbf{Q C}$ & & & $\begin{array}{ll}2 & 3 \\
\bullet & \bullet \\
\end{array}$ & $5 \quad 6$ & $\begin{array}{l}7 \\
-\end{array}$ & & & \\
\hline
\end{tabular}

WOMAC Physical Function score medium low dose

\begin{tabular}{lcccccccccccc}
\hline R & C & T & \multicolumn{2}{l}{ First author } & \multicolumn{2}{c}{ Y } & Cells & Treatment & Control & O \\
\hline S8 & 1 & a & \multicolumn{2}{c}{ Emadedin } & & \multicolumn{2}{c}{2018} & BM-MSCs & C & auto \\
\hline QC & $\mathbf{1}$ & $\mathbf{2}$ & $\mathbf{3}$ & $\mathbf{4}$ & $\mathbf{5}$ & $\mathbf{6}$ & $\mathbf{7}$ & $\mathbf{8}$ & & \\
& & $\bullet$ & $\bullet$ & $\bullet$ & $\bullet$ & $\bullet$ & $\bullet$ & $\bullet$ & & \\
\end{tabular}

WOMAC Physical Function score high dose

\begin{tabular}{|c|c|c|c|c|c|c|c|c|}
\hline $\mathbf{R}$ & $\mathbf{C}$ & $\mathbf{T}$ & First author & $\mathbf{Y}$ & Cells & Treatment & Control & $\mathbf{O}$ \\
\hline S13 & 4 & $\mathrm{a}$ & Gupta & 2016 & BM-MSCs & $\mathrm{C}+\mathrm{HA}$ & $\mathrm{S}+\mathrm{HA}$ & allo \\
\hline $\mathrm{S} 27$ & 4 & $\mathrm{a}$ & Lamo-Espinosa & 2016 & BM-MSCs & $\mathrm{C}+\mathrm{HA}$ & HA & auto \\
\hline S29 & 2 & $\mathrm{a}$ & $\mathrm{Lu}$ & 2019 & ADSCs & $\mathrm{C}$ & HA & auto \\
\hline QC & & & $\begin{array}{lll}2 & 3 & 4 \\
\bullet & \bullet & \bullet \\
\end{array}$ & $\begin{array}{ll}5 & 6\end{array}$ & $\begin{array}{l}7 \\
\bullet\end{array}$ & & & \\
\hline
\end{tabular}

WOMAC Total score low dose

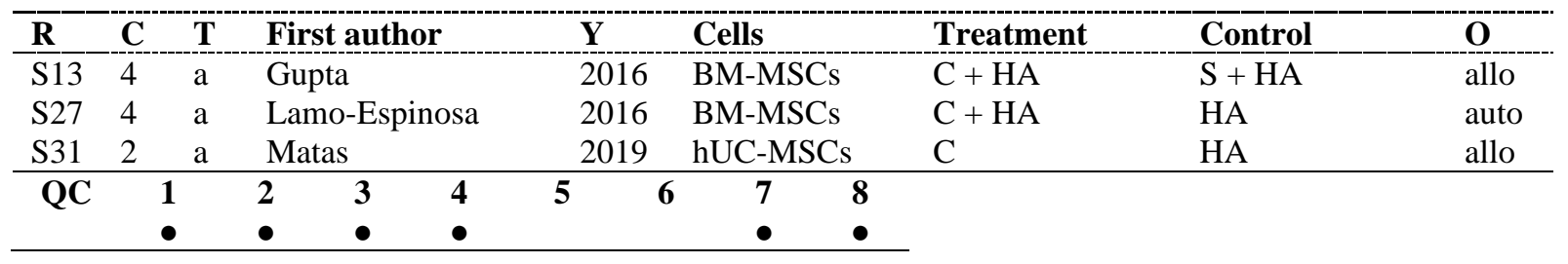


WOMAC Total score medium dose

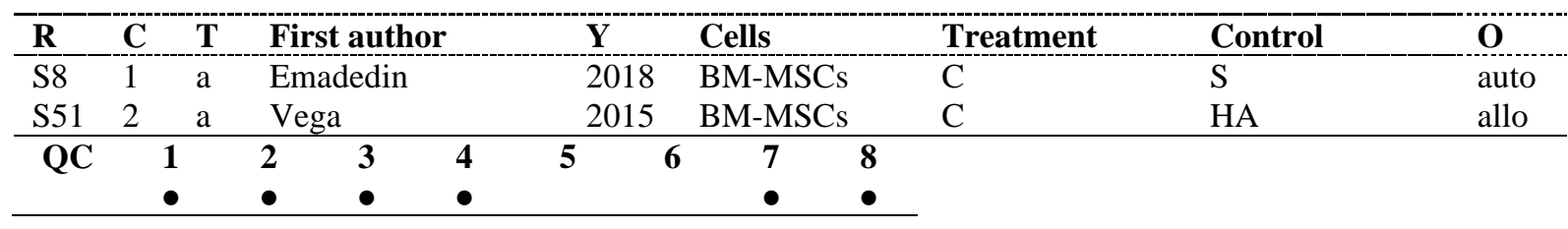

WOMAC Total score high dose

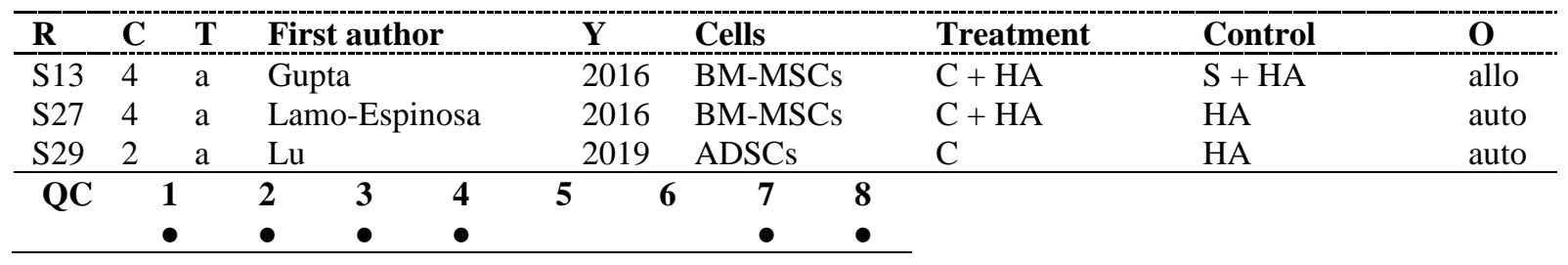

Whole-Organ Magnetic Resonance Imaging (WORMS) score reported at 6 months post treatment

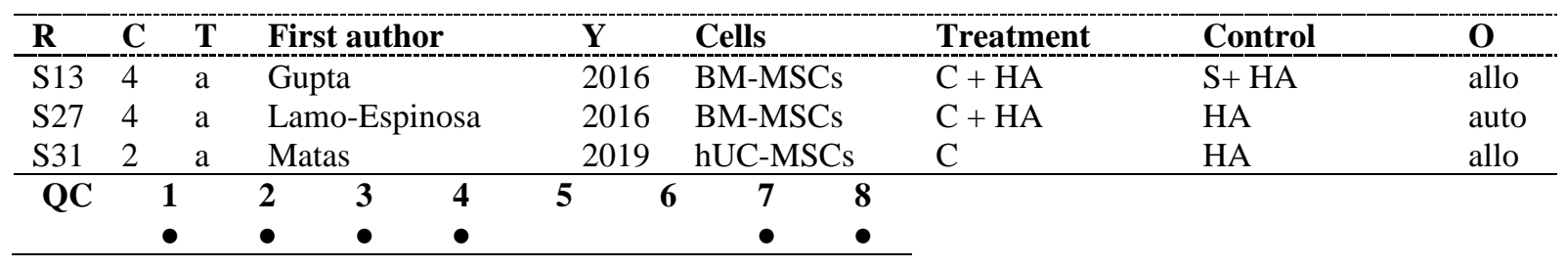

WORMS score reported at 12 months post treatment

\begin{tabular}{|c|c|c|c|c|c|c|c|c|}
\hline $\mathbf{R}$ & $\mathbf{C}$ & $\mathbf{T}$ & First author & $\mathbf{Y}$ & Cells & Treatment & Control & $\mathbf{O}$ \\
\hline $\mathrm{S} 13$ & 4 & $\mathrm{a}$ & Gupta & 2016 & BM-MSCs & $\mathrm{C}+\mathrm{HA}$ & $\mathrm{S}+\mathrm{HA}$ & allo \\
\hline S27 & 4 & $\mathrm{a}$ & Lamo-Espinosa & 2016 & BM-MSCs & $\mathrm{C}+\mathrm{HA}$ & HA & auto \\
\hline S31 & 2 & $\mathrm{a}$ & Matas & 2019 & hUC-MSCs & $\mathrm{C}$ & HA & allo \\
\hline QC & & & $\begin{array}{lll}2 & 3 & 4 \\
\bullet & \bullet & \bullet\end{array}$ & $5 \quad 6$ & $\begin{array}{ll}7 & 8 \\
\bullet & \end{array}$ & & & \\
\hline
\end{tabular}

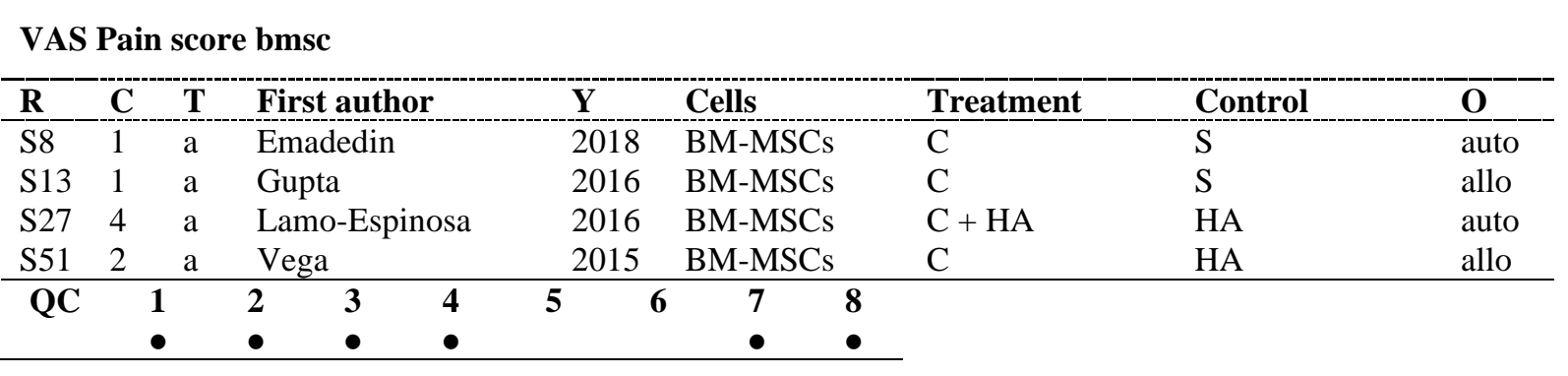


VAS Pain score admsc and ucmsc

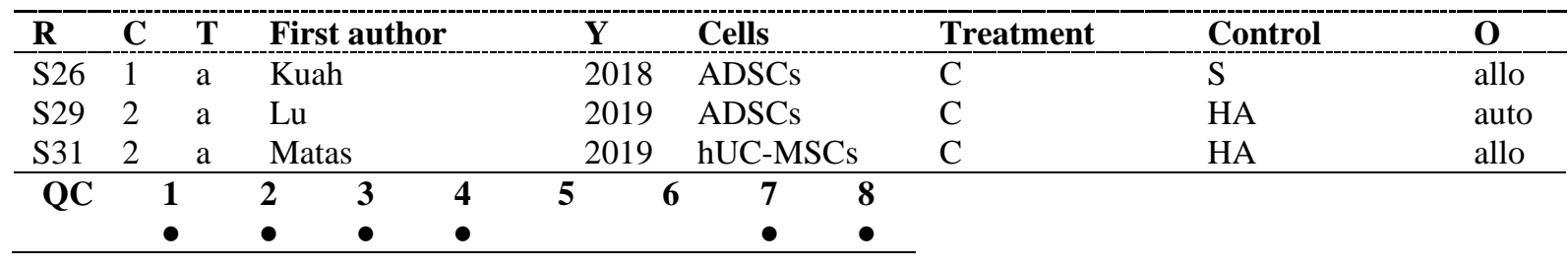

WOMAC Pain score bmsc

\begin{tabular}{|c|c|c|c|c|c|c|c|c|}
\hline $\mathbf{R}$ & $\mathbf{C}$ & $\mathbf{T}$ & First author & $\mathbf{Y}$ & Cells & Treatment & Control & $\mathbf{O}$ \\
\hline $\mathrm{S} 8$ & 1 & $\mathrm{a}$ & Emadedin & 2018 & BM-MSCs & $\mathrm{C}$ & $\mathrm{S}$ & auto \\
\hline $\mathrm{S} 13$ & 1 & $\mathrm{a}$ & Gupta & 2016 & BM-MSCs & $\mathrm{C}$ & $\mathrm{S}$ & allo \\
\hline $\mathrm{S} 27$ & 4 & $\mathrm{a}$ & Lamo-Espinosa & 2016 & BM-MSCs & $\mathrm{C}+\mathrm{HA}$ & HA & auto \\
\hline S51 & 2 & $\mathrm{a}$ & Vega & 2015 & BM-MSCs & $\mathrm{C}$ & $\mathrm{HA}$ & allo \\
\hline QC & & & $\begin{array}{ll}2 & 3 \\
\bullet & \bullet\end{array}$ & $5 \quad 6$ & $\begin{array}{l}7 \\
\bullet\end{array}$ & & & \\
\hline
\end{tabular}

\section{WOMAC Pain score admsc and ucmsc}

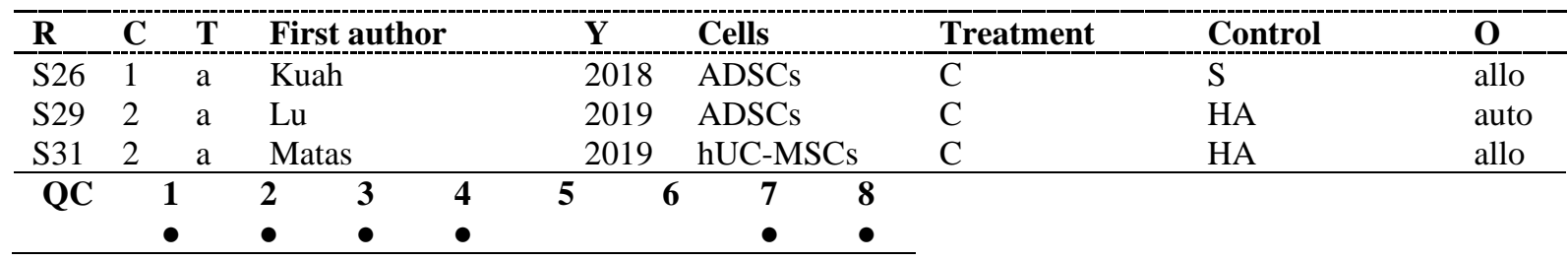

\section{VAS Pain score allogenic}

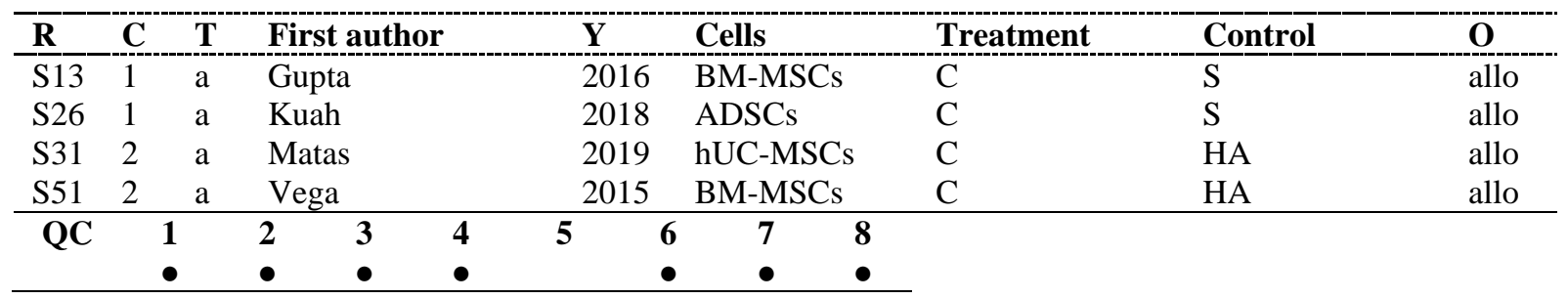

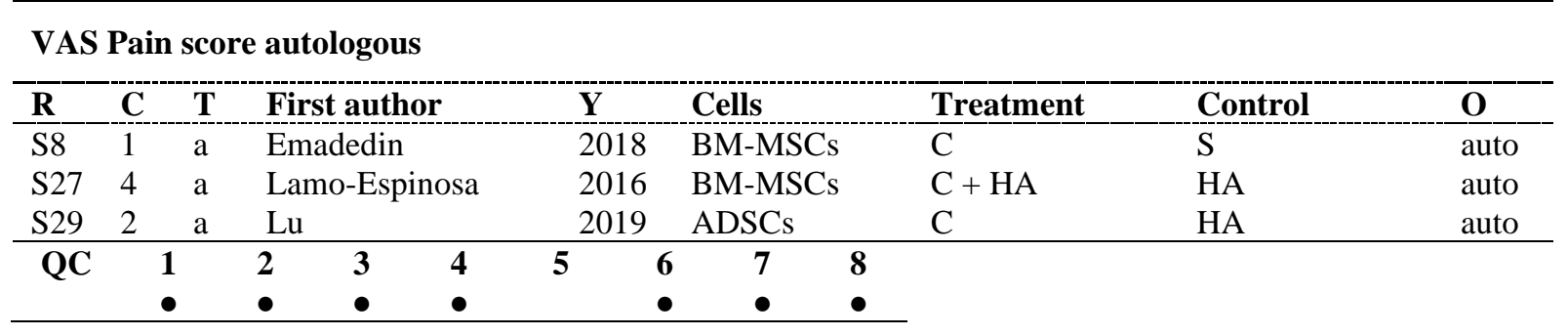




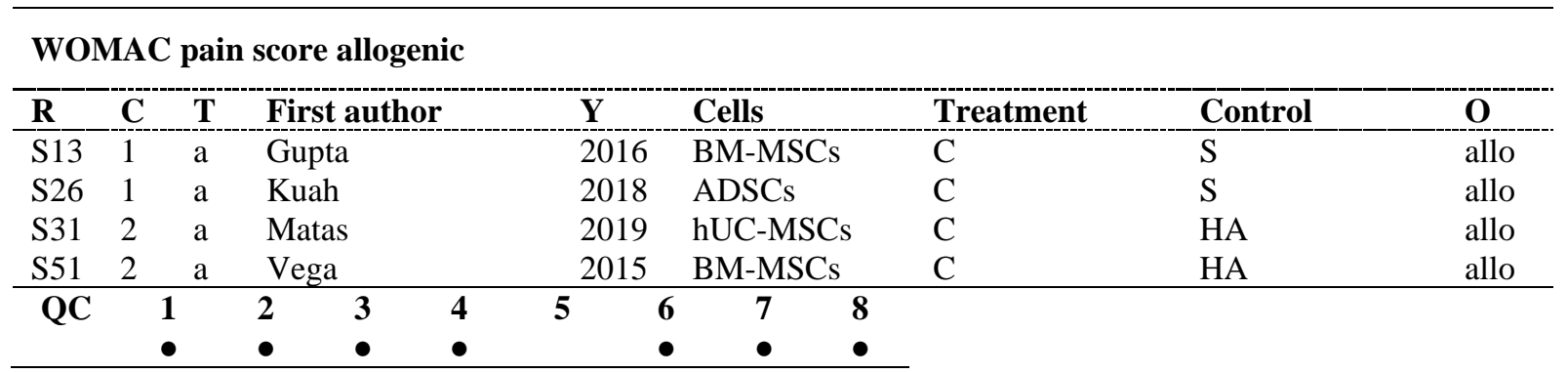

\section{WOMAC score autologous}

\begin{tabular}{|c|c|c|c|c|c|c|c|c|}
\hline $\mathbf{R}$ & C & $\mathbf{T}$ & First author & Y & Cells & Treatment & Control & O \\
\hline $\mathrm{S} 8$ & 1 & $\mathrm{a}$ & Emadedin & 2018 & BM-MSCs & $\mathrm{C}$ & $\mathrm{S}$ & auto \\
\hline S27 & 4 & $\mathrm{a}$ & Lamo-Espinosa & 2016 & BM-MSCs & $\mathrm{C}+\mathrm{HA}$ & HA & auto \\
\hline S29 & 2 & $\mathrm{a}$ & $\mathrm{Lu}$ & 2019 & ADSCs & $\mathrm{C}$ & HA & auto \\
\hline QC & & & $\begin{array}{ll}3 & 4 \\
\bullet & \bullet \\
\end{array}$ & 5 & $\bullet$ & & & \\
\hline
\end{tabular}


Table S13 | Details of the sub-analyses performed in a meta-analysis by Jeyaraman et al. ${ }^{27}$.

Abbreviations: allo $=$ allogeneic cells; auto $=$ autologous cells; $\mathrm{C}=$ category of study as outlined in Table S2; $\mathrm{O}=$ origin of cells; $\mathrm{QC}=$ quality criteria (outlined in Table 1 in the main text; a point indicates that the corresponding quality criterion was fulfilled); $\mathrm{R}=$ reference number; $\mathrm{T}=$ type of study as outlined in Table S3; Y = year of publication. The abbreviations of the cell types and treatments are provided in Table S6.

VAS Pain score reported at 6 months post treatment using bone marrow-derived cells

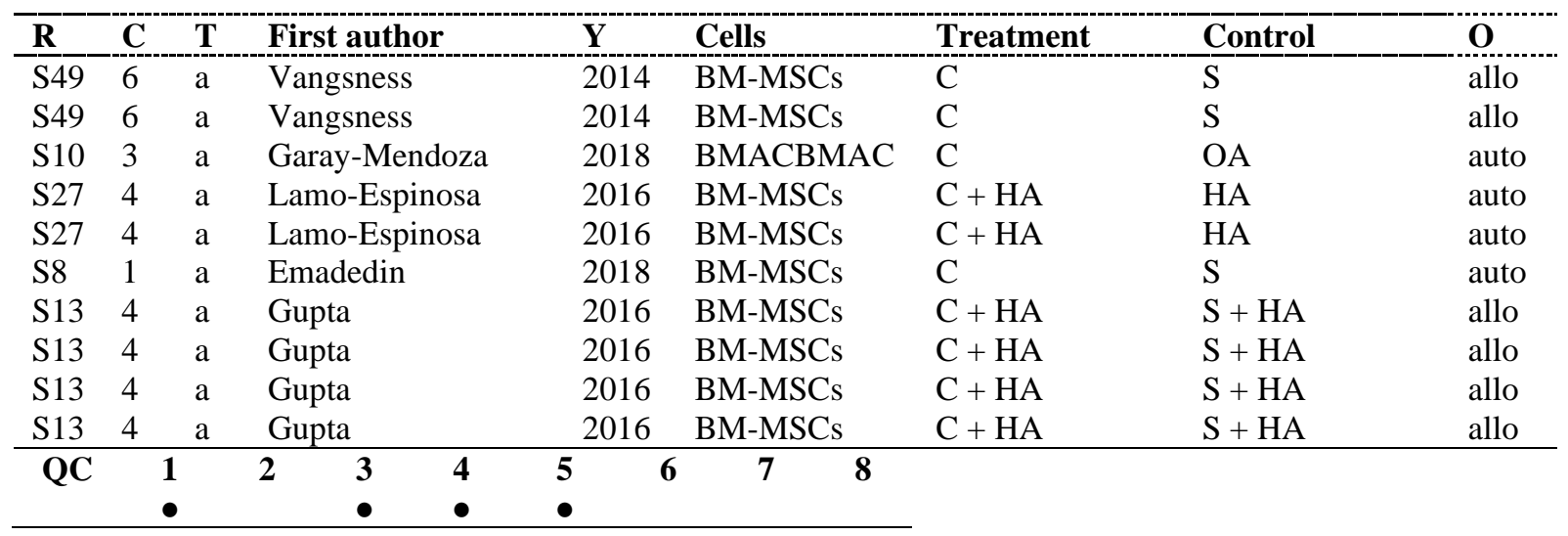

VAS Pain score reported at 12 months post treatment using bone marrow-derived cells

\begin{tabular}{|c|c|c|c|c|c|c|c|c|}
\hline $\mathbf{R}$ & $\mathbf{C}$ & $\mathbf{T}$ & First author & $\mathbf{Y}$ & Cells & Treatment & Control & $\mathbf{O}$ \\
\hline S49 & 6 & $a$ & Vangsness & 2014 & BM-MSCs & $\mathrm{C}$ & $\mathrm{S}$ & allo \\
\hline S49 & 6 & $\mathrm{a}$ & Vangsness & 2014 & BM-MSCs & $\mathrm{C}$ & S & allo \\
\hline S27 & 4 & $\mathrm{a}$ & Lamo-Espinosa & 2016 & BM-MSCs & $\mathrm{C}+\mathrm{HA}$ & $\mathrm{HA}$ & auto \\
\hline S27 & 4 & $\mathrm{a}$ & Lamo-Espinosa & 2016 & BM-MSCs & $\mathrm{C}+\mathrm{HA}$ & HA & auto \\
\hline $\mathrm{S} 13$ & 4 & $\mathrm{a}$ & Gupta & 2016 & BM-MSCs & $\mathrm{C}+\mathrm{HA}$ & $\mathrm{S}+\mathrm{HA}$ & allo \\
\hline $\mathrm{S} 13$ & 4 & a & Gupta & 2016 & BM-MSCs & $\mathrm{C}+\mathrm{HA}$ & $\mathrm{S}+\mathrm{HA}$ & allo \\
\hline S13 & 4 & $\mathrm{a}$ & Gupta & 2016 & BM-MSCs & $\mathrm{C}+\mathrm{HA}$ & $\mathrm{S}+\mathrm{HA}$ & allo \\
\hline $\mathrm{S} 13$ & 4 & $\mathrm{a}$ & Gupta & 2016 & BM-MSCs & $\mathrm{C}+\mathrm{HA}$ & $\mathrm{S}+\mathrm{HA}$ & allo \\
\hline QC & & & $\begin{array}{ll}2 & 3 \\
& \bullet \\
\end{array}$ & $\begin{array}{ll}5 & 6 \\
- & \\
\end{array}$ & $\begin{array}{ll}7 & 8 \\
\bullet & \end{array}$ & & & \\
\hline
\end{tabular}

VAS Pain score reported at 24 months post treatment using bone marrow-derived cells

\begin{tabular}{|c|c|c|c|c|c|c|c|c|c|}
\hline $\mathbf{R}$ & $\mathbf{C}$ & $\mathbf{T}$ & First author & & $\mathbf{Y}$ & Cells & Treatment & Control & $\mathbf{O}$ \\
\hline $\mathrm{S} 49$ & 6 & $\mathrm{a}$ & Vangsness & & 2014 & BM-MSCs & $\mathrm{C}$ & $\mathrm{S}$ & allo \\
\hline S49 & 6 & $\mathrm{a}$ & Vangsness & & 2014 & BM-MSCs & $\mathrm{C}$ & $\mathrm{S}$ & allo \\
\hline QC & & & $\begin{array}{ll}2 & 3 \\
& \bullet\end{array}$ & $\begin{array}{l}4 \\
\bullet\end{array}$ & $\begin{array}{ll}5 & 6 \\
\bullet & \bullet\end{array}$ & $\begin{array}{l}7 \\
\bullet\end{array}$ & & & \\
\hline
\end{tabular}


VAS Pain score reported at 6 months post treatment using adipose-derived cells

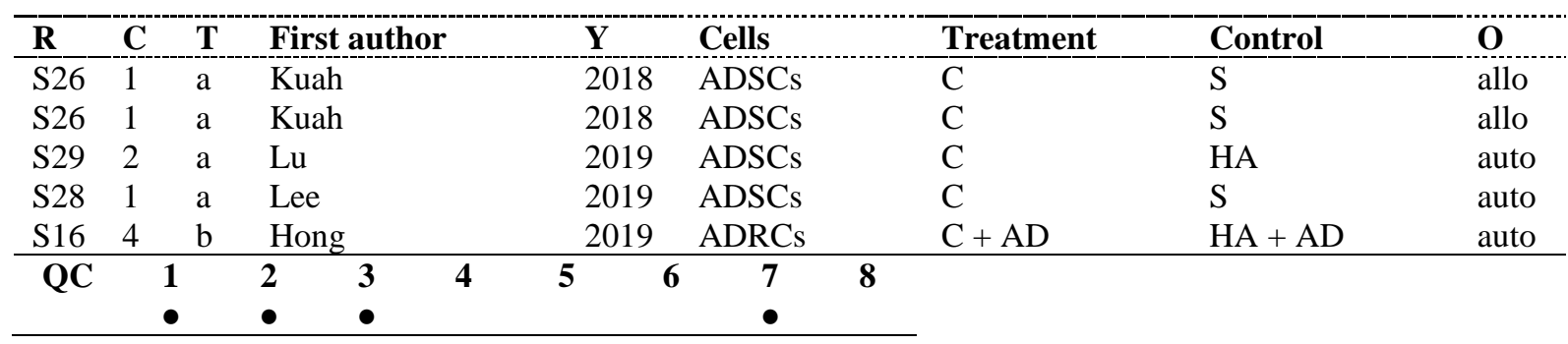

VAS Pain score reported at 12 months post treatment using adipose-derived cells

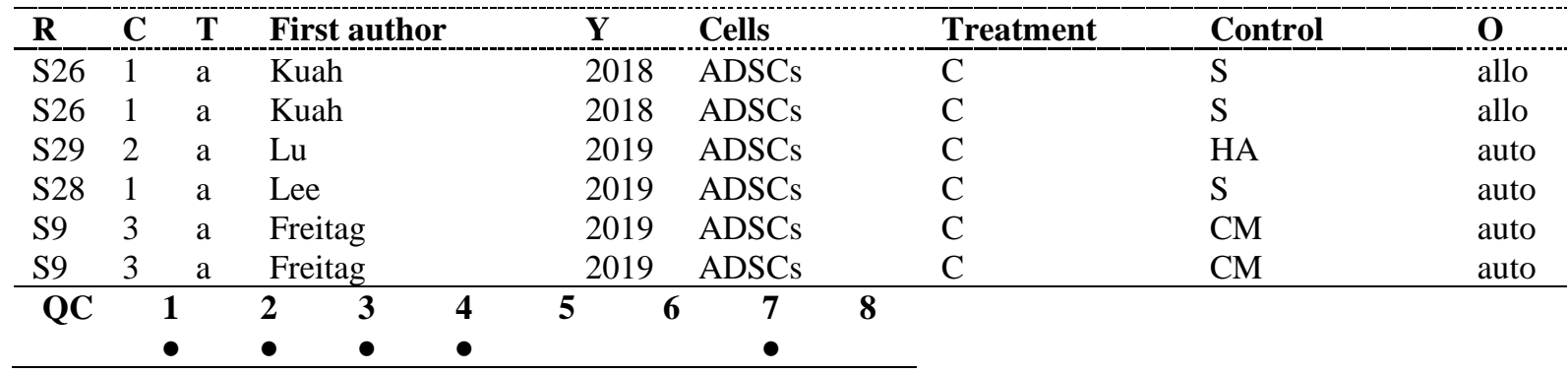

VAS Pain score reported at 24 months post treatment using adipose-derived cells

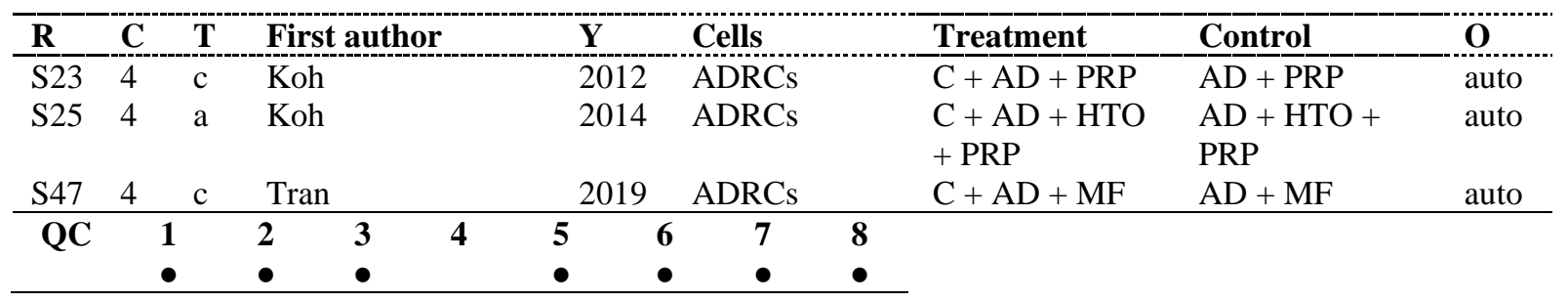

WOMAC Total score reported at 6 months post treatment using bone marrow-derived cells

\begin{tabular}{|c|c|c|c|c|c|c|c|c|}
\hline $\mathbf{R}$ & $\mathbf{C}$ & $\mathbf{T}$ & First author & $\mathbf{Y}$ & Cells & Treatment & Control & $\mathbf{O}$ \\
\hline $\mathrm{S} 10$ & 3 & $\mathrm{a}$ & Garay-Mendoza & 2018 & BMACBMAC & $\mathrm{C}$ & $\mathrm{OA}$ & auto \\
\hline $\mathrm{S} 27$ & 4 & $\mathrm{a}$ & Lamo-Espinosa & 2016 & BM-MSCs & $\mathrm{C}+\mathrm{HA}$ & HA & auto \\
\hline S27 & 4 & $\mathrm{a}$ & Lamo-Espinosa & 2016 & BM-MSCs & $\mathrm{C}+\mathrm{HA}$ & HA & auto \\
\hline $\mathrm{S} 30$ & & & $\mathrm{Lv}$ & 2015 & BM-MSCs & $?$ & $?$ & auto \\
\hline S8 & 1 & $\mathrm{a}$ & Emadedin & 2018 & BM-MSCs & $\mathrm{C}$ & $\mathrm{S}$ & auto \\
\hline \multirow[t]{2}{*}{ QC } & & & 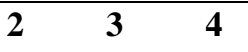 & 5 & $\begin{array}{ll}7 & 8\end{array}$ & & & \\
\hline & & & $\bullet$ & $\bullet$ & & & & \\
\hline
\end{tabular}


WOMAC Total score reported at 12 months post treatment using bone marrow-derived cells

\begin{tabular}{|c|c|c|c|c|c|c|c|c|}
\hline $\mathbf{R}$ & $\mathbf{C}$ & $\mathbf{T}$ & First author & $\mathbf{Y}$ & Cells & Treatment & Control & $\mathbf{O}$ \\
\hline $\mathrm{S} 27$ & 4 & $\mathrm{a}$ & Lamo-Espinosa & 2016 & BM-MSCs & $\mathrm{C}+\mathrm{HA}$ & HA & auto \\
\hline $\mathrm{S} 27$ & 4 & $\mathrm{a}$ & Lamo-Espinosa & 2016 & BM-MSCs & $\mathrm{C}+\mathrm{HA}$ & HA & auto \\
\hline $\mathrm{S} 30$ & & & $\mathrm{Lv}$ & 2015 & BM-MSCs & $?$ & $?$ & auto \\
\hline S13 & 4 & a & Gupta & 2016 & BM-MSCs & $\mathrm{C}+\mathrm{HA}$ & $\mathrm{S}+\mathrm{HA}$ & allo \\
\hline S13 & 4 & $\mathrm{a}$ & Gupta & 2016 & BM-MSCs & $\mathrm{C}+\mathrm{HA}$ & $\mathrm{S}+\mathrm{HA}$ & allo \\
\hline S13 & 4 & $\mathrm{a}$ & Gupta & 2016 & BM-MSCs & $\mathrm{C}+\mathrm{HA}$ & $\mathrm{S}+\mathrm{HA}$ & allo \\
\hline S13 & 4 & $\mathrm{a}$ & Gupta & 2016 & BM-MSCs & $\mathrm{C}+\mathrm{HA}$ & $\mathrm{S}+\mathrm{HA}$ & allo \\
\hline S51 & 2 & $\mathrm{a}$ & Vega & 2015 & BM-MSCs & $\mathrm{C}+\mathrm{HA}$ & HA & allo \\
\hline \multirow[t]{2}{*}{ QC } & & & 23 & $5 \quad 6$ & 78 & & & \\
\hline & & & $\bullet$ & & $\bullet$ & & & \\
\hline
\end{tabular}

WOMAC Total score reported at 6 months post treatment using adipose-derived cells

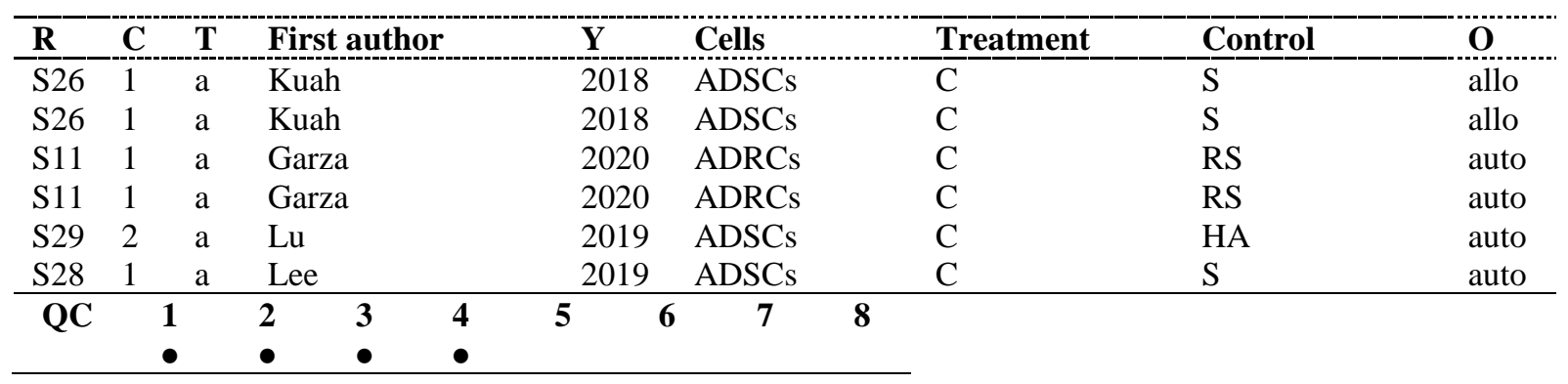

\section{WOMAC Total score reported at 12 months post treatment using adipose-derived cells}

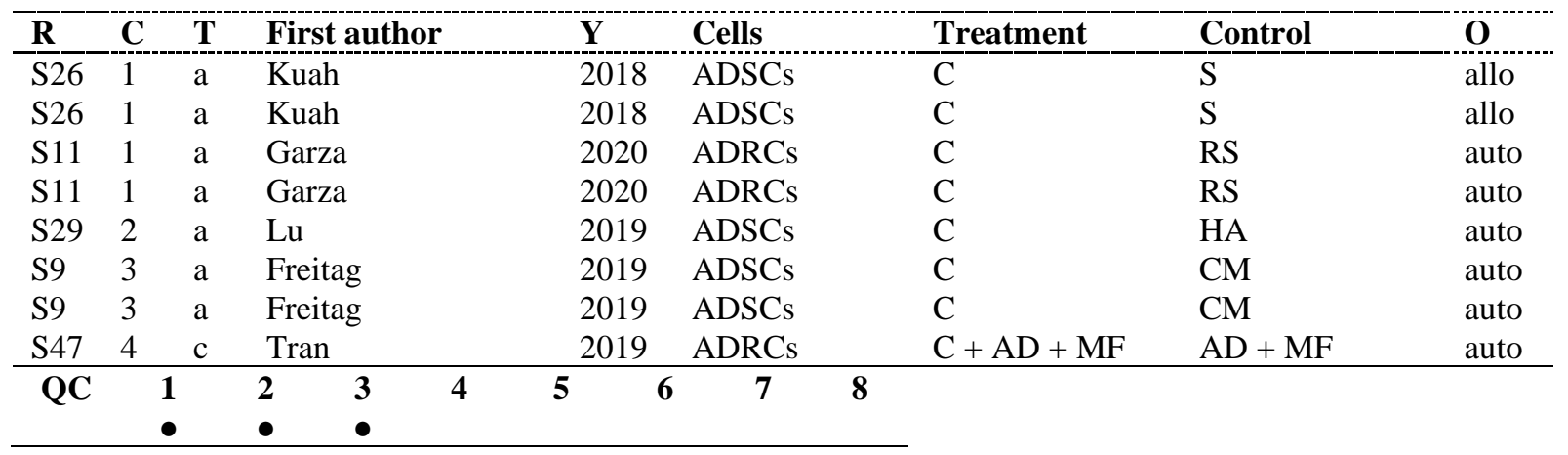

Lysholm score reported at 12 months post treatment using bone marrow-derived cells

\begin{tabular}{|c|c|c|c|c|c|c|c|c|}
\hline $\mathbf{R}$ & $\mathbf{C}$ & $\mathbf{T}$ & First author & $\mathbf{Y}$ & Cells & Treatment & Control & O \\
\hline S49 & 6 & $\mathrm{a}$ & Vangsness & 2014 & BM-MSCs & $\mathrm{C}$ & $\mathrm{S}$ & allo \\
\hline S49 & 6 & $\mathrm{a}$ & Vangsness & 2014 & BM-MSCs & $\mathrm{C}$ & S & allo \\
\hline S53 & 4 & $\mathrm{a}$ & Wong & 2013 & BM-MSCs & $\begin{array}{l}\mathrm{C}+\mathrm{MF}+\mathrm{HTO} \\
+\mathrm{HA}\end{array}$ & $\begin{array}{l}\mathrm{MF}+\mathrm{HTO}+ \\
\mathrm{HA}\end{array}$ & auto \\
\hline
\end{tabular}

\begin{tabular}{lllllllll}
\hline QC & 1 & 2 & 3 & 4 & 5 & 6 & 7 & 8 \\
& $\bullet$ & & $\bullet$ & $\bullet$ & $\bullet$ & & $\bullet$ & \\
\hline
\end{tabular}


Lysholm score reported at 24 months post treatment using bone marrow-derived cells

\begin{tabular}{|c|c|c|c|c|c|c|c|c|}
\hline $\mathbf{R}$ & $\mathbf{C}$ & $\mathbf{T}$ & First author & $\mathbf{Y}$ & Cells & Treatment & Control & $\mathbf{O}$ \\
\hline S49 & 6 & $\mathrm{a}$ & Vangsness & 2014 & BM-MSCs & $\mathrm{C}$ & $\mathrm{S}$ & allo \\
\hline S49 & 6 & $\mathrm{a}$ & Vangsness & 2014 & & $\mathrm{C}$ & S & allo \\
\hline S53 & 4 & $\mathrm{a}$ & Wong & 2013 & BM-MSCs & $\begin{array}{l}\mathrm{C}+\mathrm{MF}+\mathrm{HTO} \\
+\mathrm{HA}\end{array}$ & $\begin{array}{l}\mathrm{MF}+\mathrm{HTO}+ \\
\mathrm{HA}\end{array}$ & auto \\
\hline
\end{tabular}

\begin{tabular}{lllllllll}
\hline QC & 1 & 2 & 3 & 4 & 5 & 6 & 7 & 8 \\
& $\bullet$ & & $\bullet$ & $\bullet$ & $\bullet$ & & $\bullet$ & \\
\hline
\end{tabular}

Lysholm score reported at 24 months post treatment using adipose-derived cells

\begin{tabular}{|c|c|c|c|c|c|c|c|c|}
\hline $\mathbf{R}$ & $\mathbf{C}$ & $\mathbf{T}$ & First author & $\mathbf{Y}$ & Cells & Treatment & Control & O \\
\hline S47 & 4 & $\mathrm{c}$ & Tran & 2019 & ADRCs & $\mathrm{C}+\mathrm{AD}+\mathrm{MF}$ & $\mathrm{AD}+\mathrm{MF}$ & auto \\
\hline S23 & 4 & $\mathrm{c}$ & Koh & 2012 & ADRCs & $\mathrm{C}+\mathrm{AD}+\mathrm{PRP}$ & $A D+P R P$ & auto \\
\hline S25 & 4 & $\mathrm{a}$ & Koh & 2014 & ADRCs & $\begin{array}{l}\mathrm{C}+\mathrm{AD}+\mathrm{HTO} \\
+\mathrm{PRP}\end{array}$ & $\begin{array}{l}\mathrm{AD}+\mathrm{HTO}+ \\
\mathrm{PRP}\end{array}$ & auto \\
\hline
\end{tabular}

\begin{tabular}{ccccccccc}
\hline QC & 1 & 2 & 3 & 4 & 5 & 6 & 7 & 8 \\
& $\bullet$ & $\bullet$ & $\bullet$ & & $\bullet$ & $\bullet$ & $\bullet$ & $\bullet$ \\
\hline
\end{tabular}

KOOS score reported at 12 months post treatment using bone marrow-derived cells

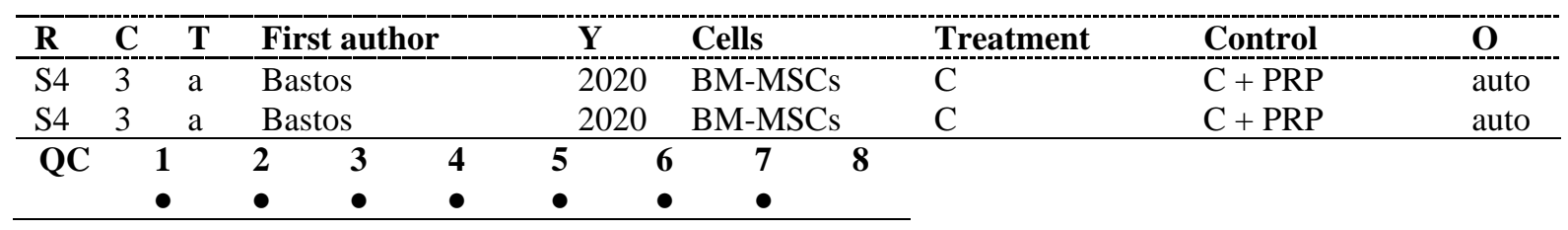

KOOS score reported at 12 months post treatment using adipose-derived cells

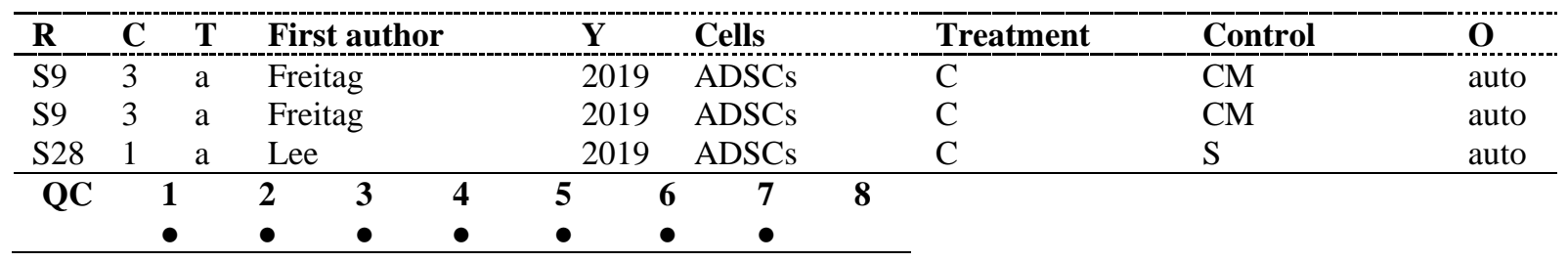

Whole-Organ Magnetic Resonance Imaging (WORMS) score reported at 12 months post treatment using bone marrow-derived cells

\begin{tabular}{|c|c|c|c|c|c|c|c|c|}
\hline $\mathbf{R}$ & C & $\mathbf{T}$ & First author & $\mathbf{Y}$ & Cells & Treatment & Control & $\mathbf{O}$ \\
\hline S27 & 4 & $\mathrm{a}$ & Lamo-Espinosa & 2016 & BM-MSCs & $\mathrm{C}+\mathrm{HA}$ & HA & auto \\
\hline $\mathrm{S} 27$ & 4 & $\mathrm{a}$ & Lamo-Espinosa & 2016 & BM-MSCs & $\mathrm{C}+\mathrm{HA}$ & $\mathrm{HA}$ & auto \\
\hline S53 & 4 & a & Wong & 2013 & BM-MSCs & $\begin{array}{l}\mathrm{C}+\mathrm{MF}+\mathrm{HTO} \\
+\mathrm{HA}\end{array}$ & $\begin{array}{l}\mathrm{MF}+\mathrm{HTO}+ \\
\mathrm{HA}\end{array}$ & auto \\
\hline $\mathrm{S} 13$ & 4 & $\mathrm{a}$ & Gupta & 2016 & BM-MSCs & $\mathrm{C}+\mathrm{HA}$ & $\mathrm{S}+\mathrm{HA}$ & allo \\
\hline $\mathrm{S} 13$ & 4 & $\mathrm{a}$ & Gupta & 2016 & BM-MSCs & $\mathrm{C}+\mathrm{HA}$ & $\mathrm{S}+\mathrm{HA}$ & allo \\
\hline QC & & b & $\begin{array}{ll}2 & 3 \\
\bullet & \bullet \\
\end{array}$ & $\begin{array}{ll}5 & 6 \\
- & \\
\end{array}$ & $\begin{array}{ll}7 & 8 \\
\bullet & \\
\end{array}$ & & & \\
\hline
\end{tabular}


WORMS score reported at 12 months post treatment using adipose-derived cells

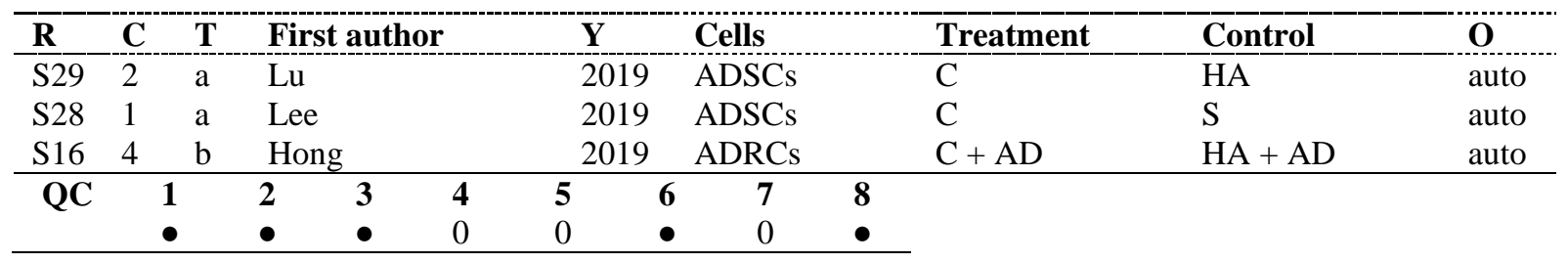

Adverse events reported after treating pkOA using bone marrow-derived cells

\begin{tabular}{|c|c|c|c|c|c|c|c|c|}
\hline $\mathbf{R}$ & $\mathbf{C}$ & $\mathbf{T}$ & First author & $\mathbf{Y}$ & Cells & Treatment & Control & $\mathbf{0}$ \\
\hline S10 & 3 & $\mathrm{a}$ & Garay-Mendoza & 2018 & BMACBMAC & $\mathrm{C}$ & $\mathrm{OA}$ & auto \\
\hline S27 & 4 & $\mathrm{a}$ & Lamo-Espinosa & 2016 & BM-MSCs & $\mathrm{C}+\mathrm{HA}$ & HA & auto \\
\hline S27 & 4 & $\mathrm{a}$ & Lamo-Espinosa & 2016 & BM-MSCs & $\mathrm{C}+\mathrm{HA}$ & HA & auto \\
\hline QC & & & $\begin{array}{lll}2 & 3 & 4 \\
\bullet & \bullet & \bullet \\
\end{array}$ & $\begin{array}{ll}5 & 6 \\
\bullet & \bullet \\
\end{array}$ & $\begin{array}{ll}7 & 8 \\
\bullet & \\
\end{array}$ & & & \\
\hline
\end{tabular}

Adverse events reported after treating pkOA using adipose-derived cells

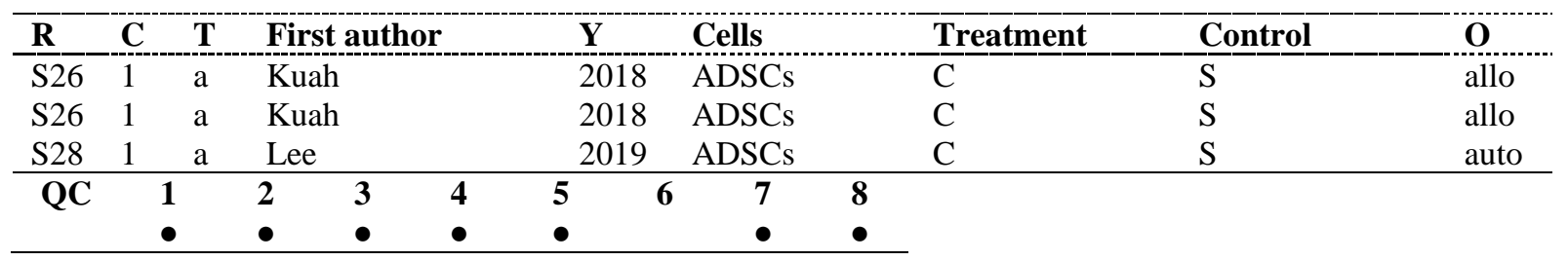


Table S14 | Details of the sub-analyses performed in a meta-analysis by Han et al. ${ }^{26}$.

Abbreviations: allo $=$ allogeneic cells; auto $=$ autologous cells; $\mathrm{C}=$ category of study as outlined in Table S2; $\mathrm{O}=$ origin of cells; $\mathrm{QC}=$ quality criteria (outlined in Table 1 in the main text; a point indicates that the corresponding quality criterion was fulfilled); $\mathrm{R}=$ reference number; $\mathrm{T}=$ type of study as outlined in Table S3; Y = year of publication. The abbreviations of the cell types and treatments are provided in Table S6.

VAS Pain score M6 bone marrow-derived cells

\begin{tabular}{|c|c|c|c|c|c|c|c|c|}
\hline $\mathbf{R}$ & C & $\mathbf{T}$ & First author & $\mathbf{Y}$ & Cells & Treatment & Control & $\mathbf{O}$ \\
\hline S27 & 4 & $\mathrm{a}$ & Lamo-Espinosa & 2016 & BM-MSCs & $\mathrm{C}+\mathrm{HA}$ & $\mathrm{HA}$ & auto \\
\hline S27 & 4 & $\mathrm{a}$ & Lamo-Espinosa & 2016 & BM-MSCs & $\mathrm{C}+\mathrm{HA}$ & HA & auto \\
\hline S49 & 6 & $\mathrm{a}$ & Vangsness & 2014 & BM-MSCs & $\mathrm{C}$ & $\mathrm{S}$ & allo \\
\hline S49 & 6 & $\mathrm{a}$ & Vangsness & 2014 & BM-MSCs & $\mathrm{C}$ & $\mathrm{S}$ & allo \\
\hline QC & & & $\begin{array}{ll}2 & 3 \\
& \bullet\end{array}$ & $\begin{array}{l}5 \\
\bullet\end{array}$ & $\begin{array}{l}7 \\
\bullet\end{array}$ & & & \\
\hline
\end{tabular}

VAS Pain score M6 adipose-derived cells

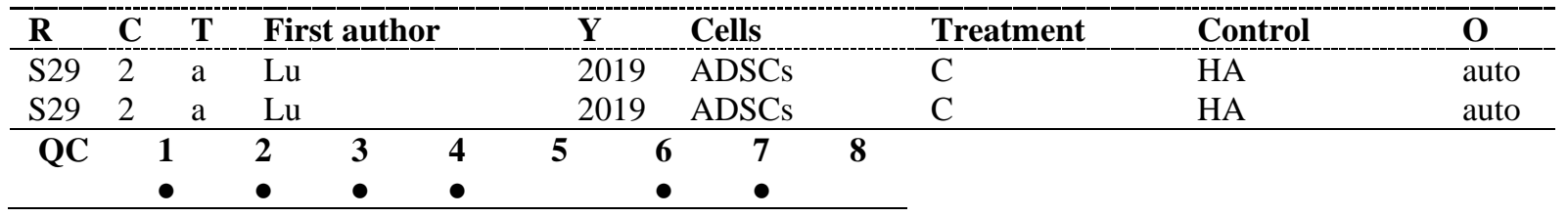

VAS Pain score M12 bone marrow-derived cells

\begin{tabular}{|c|c|c|c|c|c|c|c|c|}
\hline $\mathbf{R}$ & C & $\mathbf{T}$ & First author & $\mathbf{Y}$ & Cells & Treatment & Control & $\mathbf{O}$ \\
\hline S13 & 1 & $\mathrm{a}$ & Gupta & 2016 & BM-MSCs & $\mathrm{C}$ & $\mathrm{S}$ & allo \\
\hline S13 & 1 & $\mathrm{a}$ & Gupta & 2016 & BM-MSCs & $\mathrm{C}$ & $\mathrm{S}$ & allo \\
\hline S27 & 4 & $\mathrm{a}$ & Lamo-Espinosa & 2016 & BM-MSCs & $\mathrm{C}+\mathrm{HA}$ & HA & auto \\
\hline S27 & 4 & $\mathrm{a}$ & Lamo-Espinosa & 2016 & BM-MSCs & $\mathrm{C}+\mathrm{HA}$ & HA & auto \\
\hline S49 & 6 & $\mathrm{a}$ & Vangsness & 2014 & BM-MSCs & $\mathrm{C}$ & $\mathrm{S}$ & allo \\
\hline S49 & 6 & $\mathrm{a}$ & Vangsness & 2014 & BM-MSCs & $\mathrm{C}$ & $\mathrm{S}$ & allo \\
\hline S51 & 2 & $\mathrm{a}$ & Vega & 2015 & BM-MSCs & $\mathrm{C}$ & HA & allo \\
\hline $\mathbf{Q C}$ & & & $\begin{array}{ll}2 & 3 \\
& \bullet\end{array}$ & $\begin{array}{ll}5 & 6 \\
\bullet & \end{array}$ & $\begin{array}{l}7 \\
\bullet\end{array}$ & & & \\
\hline
\end{tabular}

VAS Pain score M12 adipose-derived cells

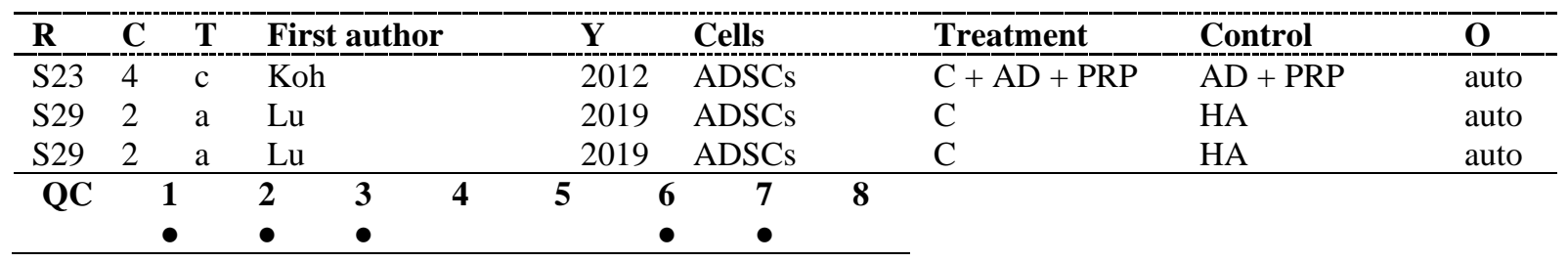


VAS Pain score M24 bone marrow-derived cells

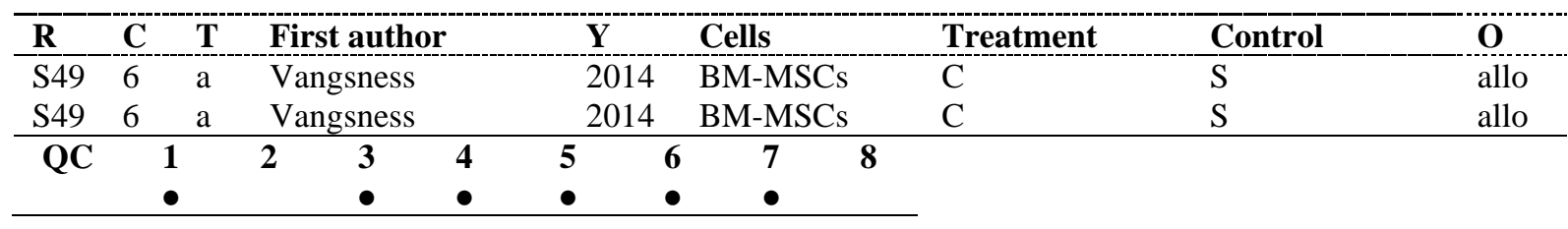

VAS Pain score M24 adipose-derived cells

\begin{tabular}{ccccccccccccc}
\hline R & C & T & \multicolumn{1}{l}{ First author } & & Y & Cells & Treatment & Control & O \\
\hline S23 & 4 & c & Koh & & & & 2012 & ADSCs & & C + AD + PRP & AD + PRP & auto \\
\hline QC & $\mathbf{1}$ & $\mathbf{2}$ & $\mathbf{3}$ & $\mathbf{4}$ & $\mathbf{5}$ & $\mathbf{6}$ & $\mathbf{7}$ & $\mathbf{8}$ & & \\
& & $\bullet$ & $\bullet$ & & $\bullet$ & $\bullet$ & $\bullet$ & $\bullet$ & & \\
\end{tabular}

WOMAC Total score at 6 months post treatment using bone marrow-derived cells

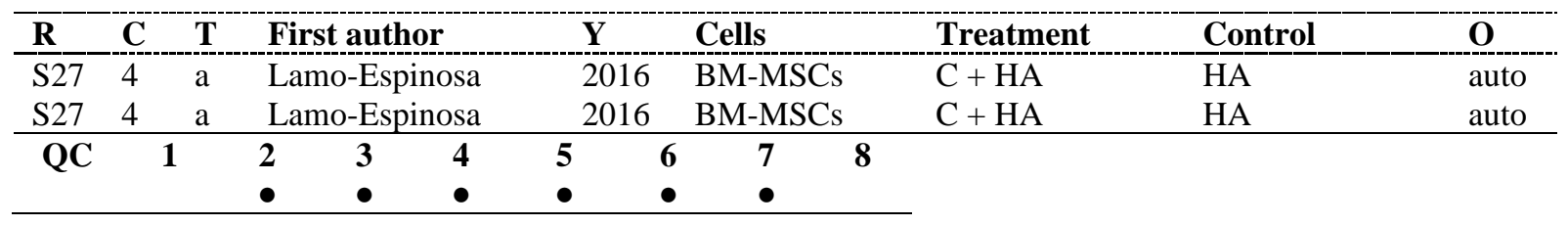

WOMAC Total score at 6 months post treatment using adipose-derived cells

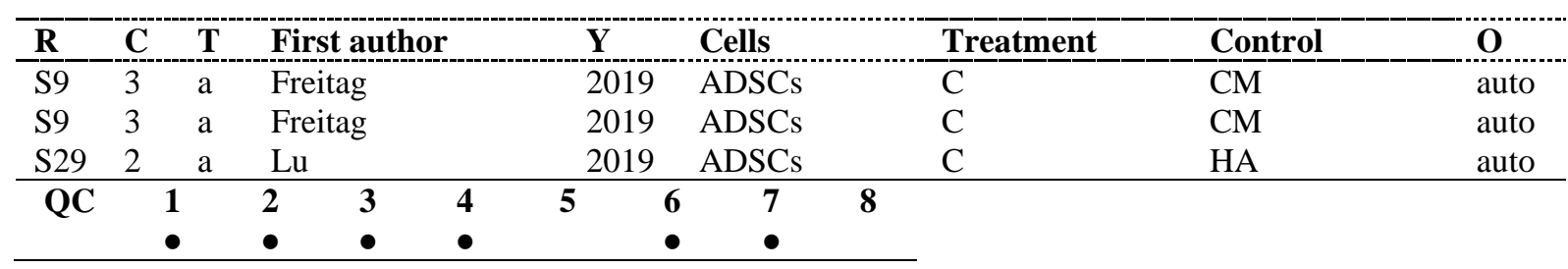

WOMAC Total score at 12 months post treatment using bone marrow-derived cells

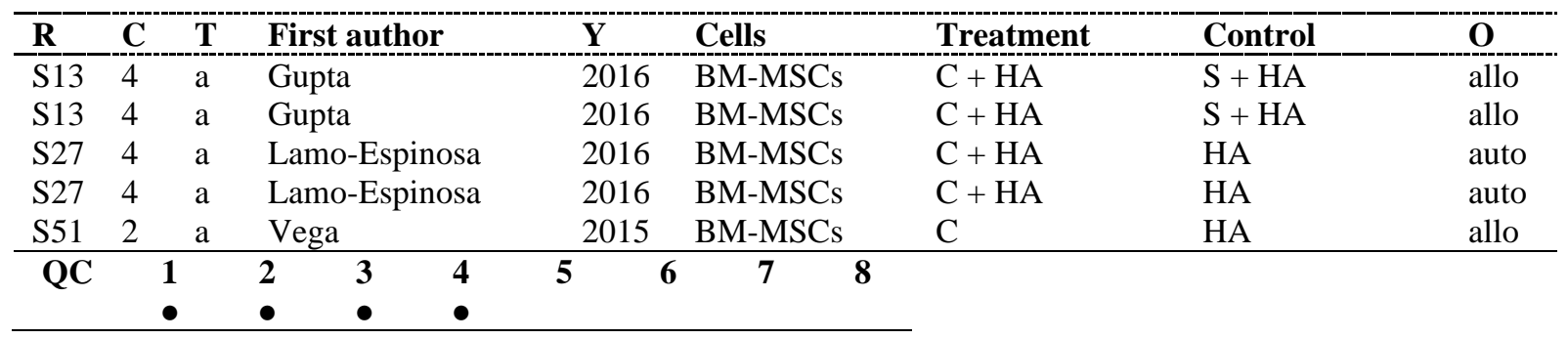


WOMAC Total score at 12 months post treatment using adipose-derived cells

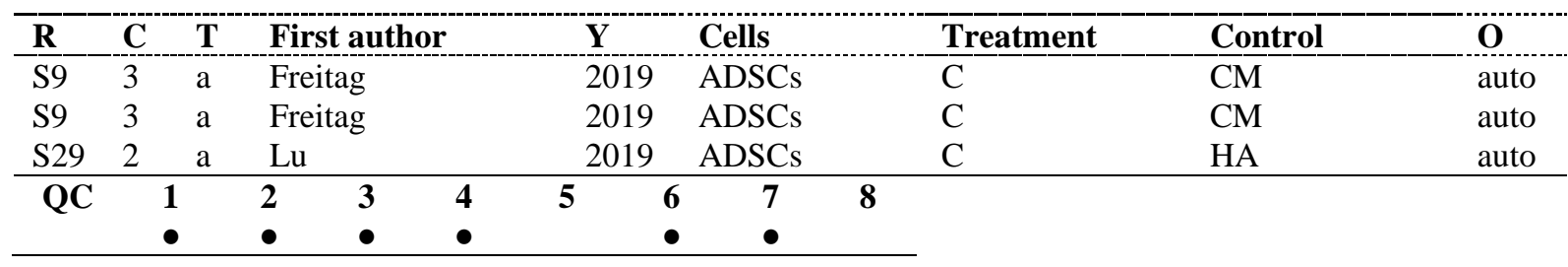

Lysholm score at 12 months post treatment using bone marrow-derived cells

\begin{tabular}{|c|c|c|c|c|c|c|c|c|}
\hline $\mathbf{R}$ & C & $\mathbf{T}$ & First author & $\mathbf{Y}$ & Cells & Treatment & Control & O \\
\hline S49 & 6 & $\mathrm{a}$ & Vangsness & 2014 & BM-MSCs & $\mathrm{C}$ & $\mathrm{S}$ & allo \\
\hline S49 & 6 & $\mathrm{a}$ & Vangsness & 2014 & BM-MSCs & $\mathrm{C}$ & $\mathrm{S}$ & allo \\
\hline S53 & 4 & $\mathrm{a}$ & Wong & 2013 & BM-MSCs & $\begin{array}{l}\mathrm{C}+\mathrm{MF}+\mathrm{HTO} \\
+\mathrm{HA}\end{array}$ & $\begin{array}{l}\mathrm{MF}+\mathrm{HTO}+ \\
\mathrm{HA}\end{array}$ & auto \\
\hline
\end{tabular}

\begin{tabular}{ccccccccc}
\hline QC & 1 & 2 & 3 & 4 & 5 & 6 & 7 & 8 \\
$\bullet$ & & $\bullet$ & $\bullet$ & $\bullet$ & $\bullet$ & & & $\bullet$ \\
\hline
\end{tabular}

Lysholm score at 12 months post treatment using adipose-derived cells

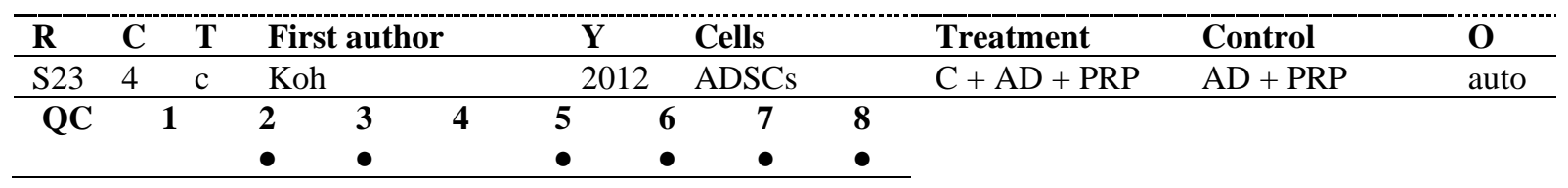

Lysholm score at 24 months post treatment using bone marrow-derived cells

\begin{tabular}{|c|c|c|c|c|c|c|c|c|}
\hline $\mathbf{R}$ & $\mathbf{C}$ & $\mathbf{T}$ & First author & $\mathbf{Y}$ & Cells & Treatment & Control & O \\
\hline S49 & 6 & $\mathrm{a}$ & Vangsness & 2014 & BM-MSCs & $\mathrm{C}$ & $\mathrm{S}$ & allo \\
\hline S49 & 6 & $\mathrm{a}$ & Vangsness & 2014 & BM-MSCs & $\mathrm{C}$ & S & allo \\
\hline S53 & 4 & $\mathrm{a}$ & Wong & 2013 & BM-MSCs & $\begin{array}{l}\mathrm{C}+\mathrm{MF}+\mathrm{HTO} \\
+\mathrm{HA}\end{array}$ & $\begin{array}{l}\mathrm{MF}+\mathrm{HTO}+ \\
\mathrm{HA}\end{array}$ & auto \\
\hline
\end{tabular}

\begin{tabular}{lllllllll}
\hline QC & 1 & 2 & 3 & 4 & 5 & 6 & 7 & 8 \\
& $\bullet$ & & $\bullet$ & $\bullet$ & $\bullet$ & $\bullet$ & & \\
\hline
\end{tabular}

Lysholm score at 24 months post treatment using adipose-derived cells

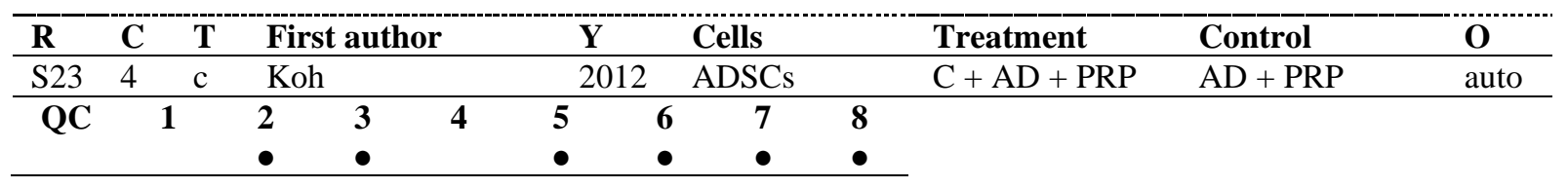


Tegner score at 12 months post treatment using bone marrow-derived cells

\begin{tabular}{lllllllllll}
\hline R & C & T & First author & Y & Cells & Treatment & Control & O \\
\hline S53 & 4 & a & Wong & & & 2013 & BM-MSCs & $\begin{array}{l}\text { C + MF + HTO } \\
+ \text { HA }\end{array}$ & $\begin{array}{l}\text { MF + HTO + } \\
\text { HA }\end{array}$ \\
\hline QC & $\mathbf{1}$ & $\mathbf{2}$ & $\mathbf{3}$ & $\mathbf{4}$ & $\mathbf{5}$ & $\mathbf{6}$ & $\mathbf{7}$ & $\mathbf{8}$ & \\
& & $\bullet$ & $\bullet$ & $\bullet$ & $\bullet$ & $\bullet$ & $\bullet$ & $\bullet$ & \\
\hline
\end{tabular}

Tegner score at 12 months post treatment using adipose-derived cells

\begin{tabular}{ccccccccccccc}
\hline R & C & T & \multicolumn{2}{l}{ First author } & & Y & Cells & Treatment & Control & O \\
\hline S23 & 4 & c & Koh & & & & 2012 & ADSCs & & C + AD + PRP & AD + PRP & auto \\
\hline QC & $\mathbf{1}$ & $\mathbf{2}$ & $\mathbf{3}$ & $\mathbf{4}$ & $\mathbf{5}$ & $\mathbf{6}$ & $\mathbf{7}$ & $\mathbf{8}$ & & \\
& & $\bullet$ & $\bullet$ & & $\bullet$ & $\bullet$ & $\bullet$ & $\bullet$ & & \\
\end{tabular}


Table S15 | Details of the sub-analyses performed in a meta-analysis by Kim et al. ${ }^{17}$.

Abbreviations: allo $=$ allogeneic cells; auto $=$ autologous cells; $\mathrm{C}=$ category of study as outlined in Table S2; $\mathrm{O}=$ origin of cells; $\mathrm{QC}=$ quality criteria (outlined in Table 1 in the main text; a point indicates that the corresponding quality criterion was fulfilled); $\mathrm{R}=$ reference number; $\mathrm{T}=$ type of study as outlined in Table S3; Y = year of publication. The abbreviations of the cell types and treatments are provided in Table S6.

\begin{tabular}{|c|c|c|c|c|c|c|c|c|}
\hline \multicolumn{9}{|c|}{ VAS Pain score } \\
\hline $\mathbf{R}$ & C & $\mathbf{T}$ & First author & $\mathbf{Y}$ & Cells & Treatment & Control & O \\
\hline S51 & 2 & $\mathrm{a}$ & Vega & 2015 & BM-MSCs & $\mathrm{C}$ & HA & allo \\
\hline S27 & 4 & $\mathrm{a}$ & Lamo-Espinosa & 2016 & BM-MSCs & $\mathrm{C}+\mathrm{HA}$ & HA & auto \\
\hline S13 & 4 & $\mathrm{a}$ & Gupta & 2016 & BM-MSCs & $\mathrm{C}+\mathrm{HA}$ & $\mathrm{S}+\mathrm{HA}$ & allo \\
\hline S26 & 1 & $\mathrm{a}$ & Kuah & 2018 & ADSCs & $\mathrm{C}$ & $\mathrm{S}$ & allo \\
\hline S8 & 1 & a & Emadedin & 2018 & BM-MSCs & $\mathrm{C}$ & $\mathrm{S}$ & auto \\
\hline$\overline{Q C}$ & & & $\begin{array}{ll}2 & 3 \\
\bullet & \bullet\end{array}$ & $5 \quad 6$ & $\begin{array}{l}7 \\
\bullet\end{array}$ & & & \\
\hline
\end{tabular}

\section{WOMAC Total score}

\begin{tabular}{|c|c|c|c|c|c|c|c|c|}
\hline $\mathbf{R}$ & C & $\mathbf{T}$ & First author & $\mathbf{Y}$ & Cells & Treatment & Control & O \\
\hline S51 & 2 & $\mathrm{a}$ & Vega & 2015 & BM-MSCs & $\mathrm{C}$ & HA & allo \\
\hline S13 & 4 & $\mathrm{a}$ & Gupta & 2016 & BM-MSCs & $\mathrm{C}+\mathrm{HA}$ & $\mathrm{S}+\mathrm{HA}$ & allo \\
\hline S27 & 4 & $\mathrm{a}$ & Lamo-Espinosa & 2016 & BM-MSCs & $\mathrm{C}+\mathrm{HA}$ & HA & auto \\
\hline S8 & 1 & $\mathrm{a}$ & Emadedin & 2018 & BM-MSCs & $\mathrm{C}$ & $\mathrm{S}$ & auto \\
\hline QC & & & $\begin{array}{ll}2 & 3 \\
\bullet & \bullet \\
\end{array}$ & 5 & $\begin{array}{l}7 \\
\bullet \\
\end{array}$ & & & \\
\hline
\end{tabular}

\section{WOMAC Pain score}

\begin{tabular}{|c|c|c|c|c|c|c|c|c|}
\hline $\mathbf{R}$ & C & $\mathbf{T}$ & First author & Y & Cells & Treatment & Control & $\mathbf{O}$ \\
\hline S8 & 1 & $\mathrm{a}$ & Emadedin & 2018 & BM-MSCs & $\mathrm{C}$ & $\mathrm{S}$ & auto \\
\hline S13 & 4 & $\mathrm{a}$ & Gupta & 2016 & BM-MSCs & $\mathrm{C}+\mathrm{HA}$ & $\mathrm{S}+\mathrm{HA}$ & allo \\
\hline S26 & 1 & $\mathrm{a}$ & Kuah & 2018 & ADSCs & $\mathrm{C}$ & $\mathrm{S}$ & allo \\
\hline S27 & 4 & $\mathrm{a}$ & Lamo-Espinosa & 2016 & BM-MSCs & $\mathrm{C}+\mathrm{HA}$ & HA & auto \\
\hline S51 & 2 & $\mathrm{a}$ & Vega & 2015 & BM-MSCs & $\mathrm{C}$ & HA & allo \\
\hline QC & & & $\begin{array}{ll}3 & 4 \\
\bullet & \bullet \\
\end{array}$ & 5 & $\begin{array}{l}7 \\
\bullet \\
\end{array}$ & & & \\
\hline
\end{tabular}

\section{WOMAC Function score}

\begin{tabular}{lllllllllll}
\hline R & C & T & First author & & Y & Cells & & Treatment & Control & O \\
\hline S8 & 1 & a & Emadedin & & 2018 & BM-MSCs & C & S & auto \\
S13 & 4 & a & Gupta & & 2016 & BM-MSCs & C + HA & S + HA & allo \\
S27 & 4 & a & Lamo-Espinosa & \multicolumn{2}{c}{2016} & BM-MSCs & C + HA & HA & auto \\
\hline QC & $\mathbf{1}$ & $\mathbf{2}$ & $\mathbf{3}$ & $\mathbf{4}$ & $\mathbf{5}$ & $\mathbf{6}$ & $\mathbf{7}$ & $\mathbf{8}$ & & \\
& $\bullet$ & $\bullet$ & $\bullet$ & $\bullet$ & $\bullet$ & & $\bullet$ & $\bullet$ & &
\end{tabular}


Whole-Organ Magnetic Resonance Imaging (WORMS) score

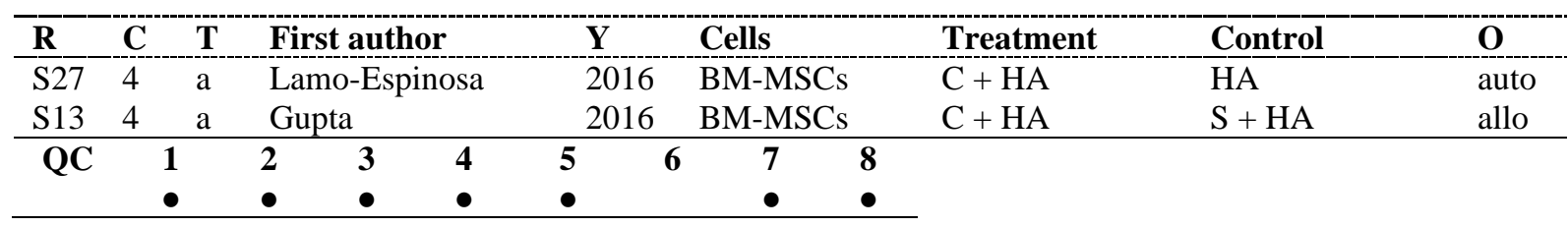

Categorical evaluations of MRI data

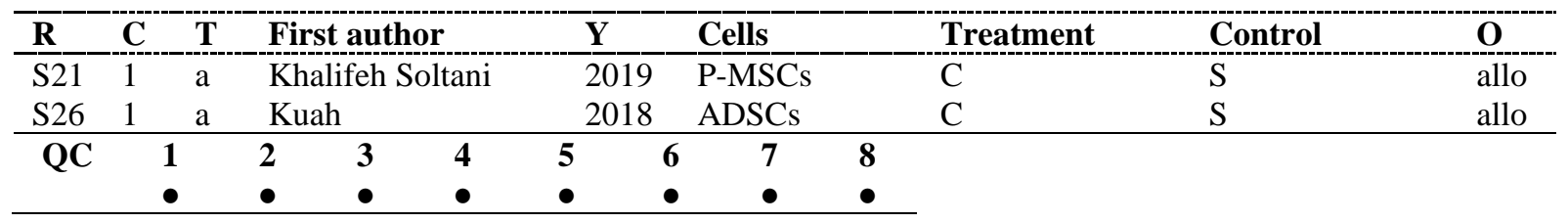

Cumulative pain scores (WOMAC and VAS): WOMAC

\begin{tabular}{|c|c|c|c|c|c|c|c|c|}
\hline $\mathbf{R}$ & C & $\mathbf{T}$ & First author & $\mathbf{Y}$ & Cells & Treatment & Control & $\mathbf{O}$ \\
\hline S51 & 2 & $\mathrm{a}$ & Vega & 2015 & BM-MSCs & $\mathrm{C}$ & $\mathrm{HA}$ & allo \\
\hline S13 & 4 & $\mathrm{a}$ & Gupta & 2016 & BM-MSCs & $\mathrm{C}+\mathrm{HA}$ & $\mathrm{S}+\mathrm{HA}$ & allo \\
\hline S27 & 4 & $\mathrm{a}$ & Lamo-Espinosa & 2016 & BM-MSCs & $\mathrm{C}+\mathrm{HA}$ & HA & auto \\
\hline S26 & 1 & $\mathrm{a}$ & Kuah & 2018 & ADSCs & $\mathrm{C}$ & S & allo \\
\hline S8 & 1 & $\mathrm{a}$ & Emadedin & 2018 & BM-MSCs & $\mathrm{C}$ & $\mathrm{S}$ & auto \\
\hline $\mathbf{Q C}$ & & & $\begin{array}{ll}2 & 3 \\
\bullet & \bullet\end{array}$ & $5 \quad 6$ & $\begin{array}{l}7 \\
\bullet\end{array}$ & & & \\
\hline
\end{tabular}

\section{Cumulative pain scores (WOMAC and VAS): VAS}

\begin{tabular}{|c|c|c|c|c|c|c|c|c|}
\hline $\mathbf{R}$ & $\mathbf{C}$ & $\mathbf{T}$ & First author & $\mathbf{Y}$ & Cells & Treatment & Control & $\mathbf{O}$ \\
\hline S51 & 2 & $\mathrm{a}$ & Vega & 2015 & BM-MSCs & $\mathrm{C}$ & HA & allo \\
\hline S13 & 4 & $\mathrm{a}$ & Gupta & 2016 & BM-MSCs & $\mathrm{C}+\mathrm{HA}$ & $\mathrm{S}+\mathrm{HA}$ & allo \\
\hline $\mathrm{S} 27$ & 4 & $\mathrm{a}$ & Lamo-Espinosa & 2016 & BM-MSCs & $\mathrm{C}+\mathrm{HA}$ & $\mathrm{HA}$ & auto \\
\hline S8 & 1 & $\mathrm{a}$ & Emadedin & 2018 & BM-MSCs & $\mathrm{C}$ & $S$ & auto \\
\hline S26 & 1 & $\mathrm{a}$ & Kuah & 2018 & ADSCs & $\mathrm{C}$ & $\mathrm{S}$ & allo \\
\hline QC & & & $\begin{array}{ll}2 & 3 \\
- & \bullet \\
\end{array}$ & $5 \quad 6$ & $\begin{array}{l}7 \\
\bullet\end{array}$ & & & \\
\hline
\end{tabular}


Table S16 | Details of the sub-analyses performed in a meta-analysis by Ding et al. ${ }^{25}$.

Abbreviations: allo $=$ allogeneic cells; auto $=$ autologous cells; $\mathrm{C}=$ category of study as outlined in Table S2; $\mathrm{O}=$ origin of cells; $\mathrm{QC}=$ quality criteria (outlined in Table 1 in the main text; a point indicates that the corresponding quality criterion was fulfilled); $\mathrm{R}=$ reference number; $\mathrm{T}=$ type of study as outlined in Table S3; Y = year of publication. The abbreviations of the cell types and treatments are provided in Table S6.

WOMAC Total score reported at 6 months post treatment using allogeneic cells

\begin{tabular}{|c|c|c|c|c|c|c|c|c|}
\hline $\mathbf{R}$ & C & $\mathbf{T}$ & First author & $\mathbf{Y}$ & Cells & Treatment & Control & O \\
\hline S31 & 2 & $\mathrm{a}$ & Matas & 2019 & hUC-MSCs & $\mathrm{C}$ & HA & allo \\
\hline S31 & 2 & $\mathrm{a}$ & Matas & 2019 & hUC-MSCs & $\mathrm{C}$ & HA & allo \\
\hline S26 & 1 & $\mathrm{a}$ & Kuah & 2018 & ADSCs & $\mathrm{C}$ & $\mathrm{S}$ & allo \\
\hline S26 & 1 & $\mathrm{a}$ & Kuah & 2018 & ADSCs & $\mathrm{C}$ & $S$ & allo \\
\hline S52 & 2 & $\mathrm{a}$ & Wang & 2016 & hUC-MSCs & $\mathrm{C}$ & HA & allo \\
\hline S13 & 4 & $\mathrm{a}$ & Gupta & 2016 & BM-MSCs & $\mathrm{C}+\mathrm{HA}$ & $\mathrm{S}+\mathrm{HA}$ & allo \\
\hline S13 & 4 & $\mathrm{a}$ & Gupta & 2016 & BM-MSCs & $\mathrm{C}+\mathrm{HA}$ & $\mathrm{S}+\mathrm{HA}$ & allo \\
\hline S13 & 4 & $\mathrm{a}$ & Gupta & 2016 & BM-MSCs & $\mathrm{C}+\mathrm{HA}$ & $\mathrm{S}+\mathrm{HA}$ & allo \\
\hline S13 & 4 & $\mathrm{a}$ & Gupta & 2016 & BM-MSCs & $\mathrm{C}+\mathrm{HA}$ & $\mathrm{S}+\mathrm{HA}$ & allo \\
\hline S51 & 2 & $\mathrm{a}$ & Vega & 2015 & BM-MSCs & $\mathrm{C}$ & HA & allo \\
\hline QC & & & $\begin{array}{ll}2 & 3 \\
\bullet & \bullet\end{array}$ & 5 & 7 & & & \\
\hline
\end{tabular}

WOMAC Total score reported at 6 months post treatment using autologous cells

\begin{tabular}{|c|c|c|c|c|c|c|c|c|}
\hline $\mathbf{R}$ & C & $\mathbf{T}$ & First author & $\mathbf{Y}$ & Cells & Treatment & Control & O \\
\hline S29 & 2 & $\mathrm{a}$ & $\mathrm{Lu}$ & 2019 & ADSCs & $\mathrm{C}$ & HA & auto \\
\hline S9 & 3 & a & Freitag & 2019 & ADSCs & $\mathrm{C}$ & $\mathrm{CM}$ & auto \\
\hline S9 & 3 & $\mathrm{a}$ & Freitag & 2019 & ADSCs & $\mathrm{C}$ & $\mathrm{CM}$ & auto \\
\hline S27 & 4 & $\mathrm{a}$ & Lamo-Espinosa & 2016 & BM-MSCs & $\mathrm{C}+\mathrm{HA}$ & HA & auto \\
\hline S27 & 4 & $\mathrm{a}$ & Lamo-Espinosa & 2016 & BM-MSCs & $\mathrm{C}+\mathrm{HA}$ & HA & auto \\
\hline S8 & 1 & $\mathrm{a}$ & Emadedin & 2018 & BM-MSCs & $\mathrm{C}$ & S & auto \\
\hline QC & & & \begin{tabular}{|ll}
2 & 3
\end{tabular} & 5 & 7 & & & \\
\hline
\end{tabular}

KOOS score reported at 12 months post treatment using allogeneic cells

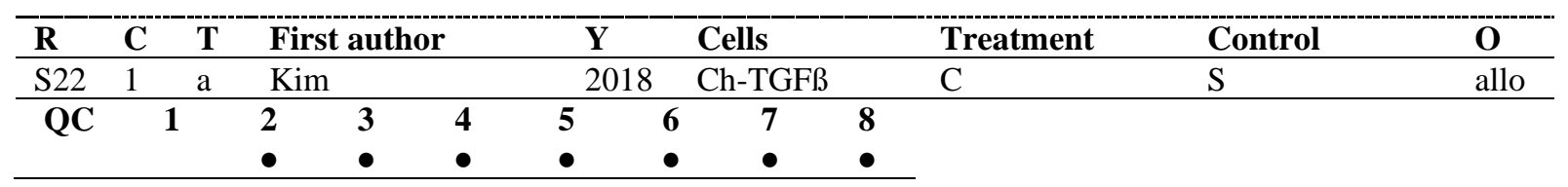

KOOS score reported at 12 months post treatment using autologous cells

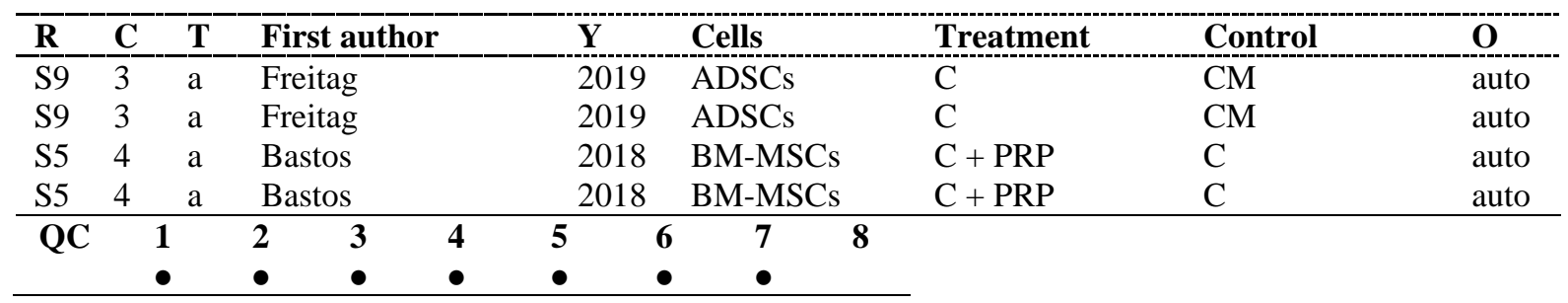


VAS Pain score reported at 6 months post treatment using allogeneic cells

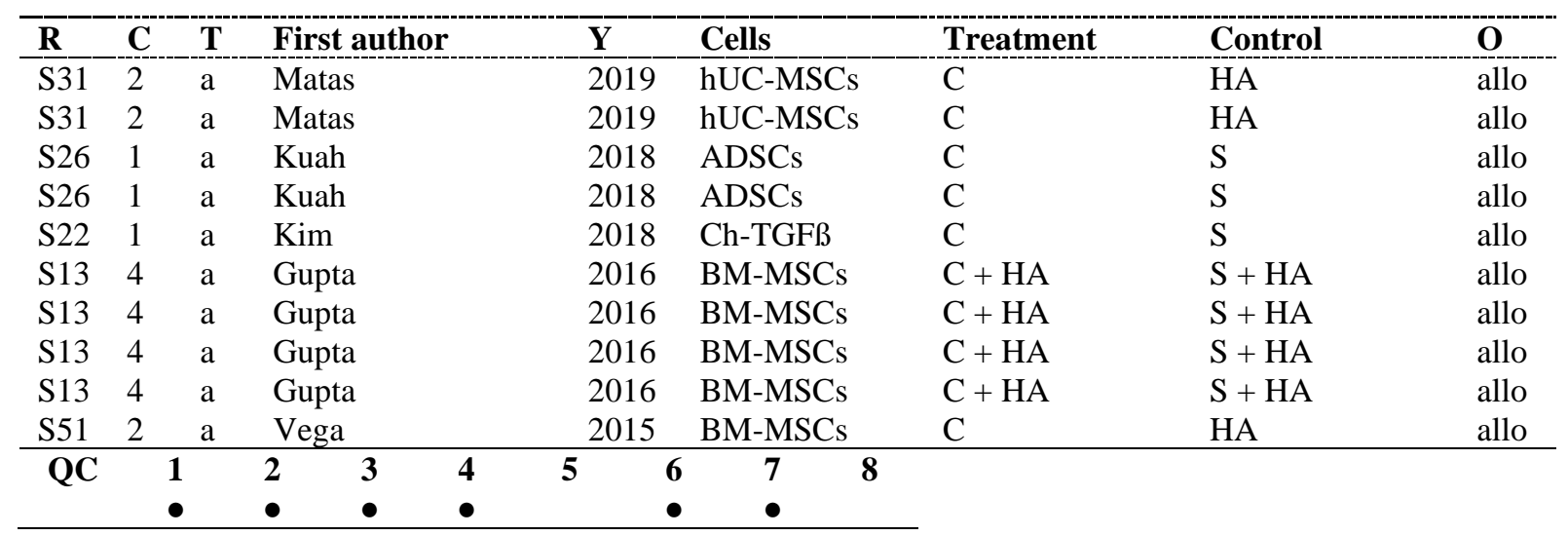

VAS Pain score reported at 6 months post treatment using autologous cells

\begin{tabular}{|c|c|c|c|c|c|c|c|c|}
\hline $\mathbf{R}$ & C & $\mathbf{T}$ & First author & $\mathbf{Y}$ & Cells & Treatment & Control & $\mathbf{O}$ \\
\hline S29 & 2 & $\mathrm{a}$ & $\mathrm{Lu}$ & 2019 & $\mathrm{ADSCs}$ & $\mathrm{C}$ & HA & auto \\
\hline S9 & 3 & $\mathrm{a}$ & Freitag & 2019 & ADSCs & $\mathrm{C}$ & $\mathrm{CM}$ & auto \\
\hline S9 & 3 & $\mathrm{a}$ & Freitag & 2019 & ADSCs & $\mathrm{C}$ & $\mathrm{CM}$ & auto \\
\hline S27 & 4 & $\mathrm{a}$ & Lamo-Espinosa & 2016 & BM-MSCs & $\mathrm{C}+\mathrm{HA}$ & HA & auto \\
\hline S27 & 4 & $\mathrm{a}$ & Lamo-Espinosa & 2016 & BM-MSCs & $\mathrm{C}+\mathrm{HA}$ & HA & auto \\
\hline S8 & 1 & $\mathrm{a}$ & Emadedin & 2018 & BM-MSCs & $\mathrm{C}$ & $\mathrm{S}$ & auto \\
\hline QC & & & $\begin{array}{ll}2 & 3 \\
\bullet & \bullet \\
\end{array}$ & $5 \quad 6$ & $\begin{array}{ll} & 8 \\
\bullet & \\
\end{array}$ & & & \\
\hline
\end{tabular}

Adverse events reported after treating pkOA using allogeneic cells

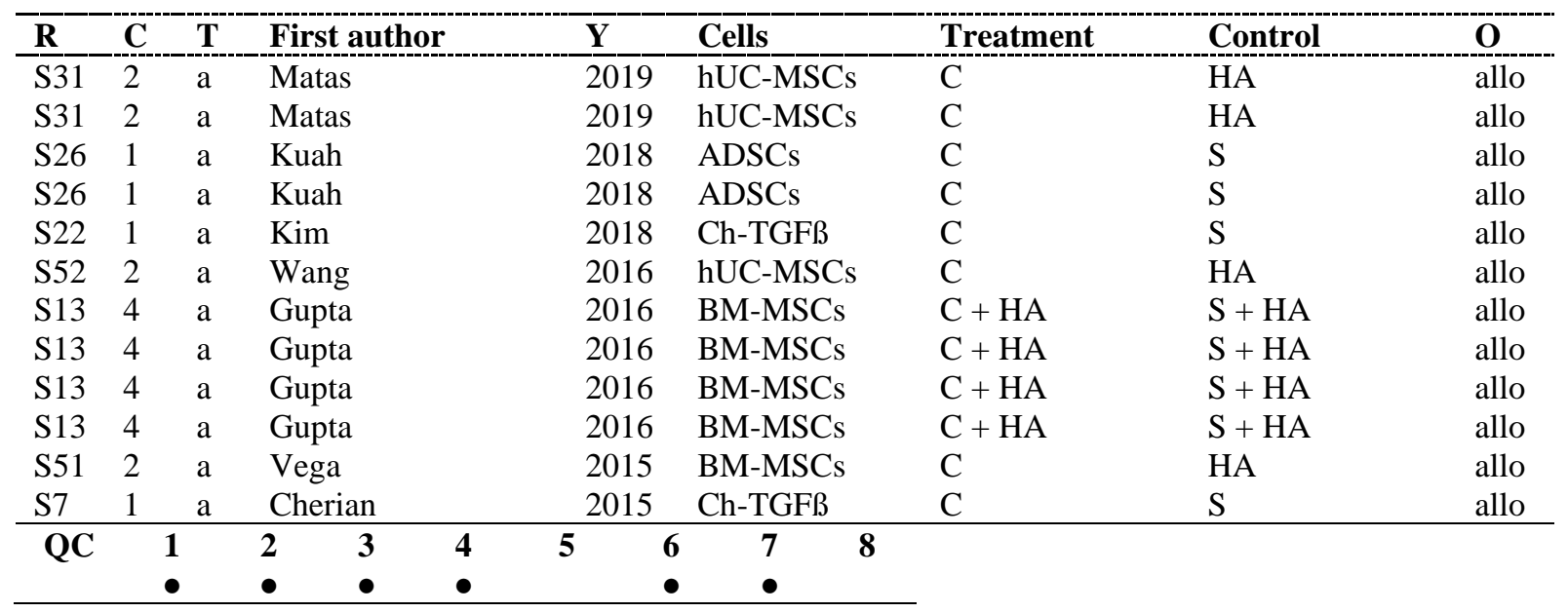


Adverse events reported after treating pkOA using autologous cells

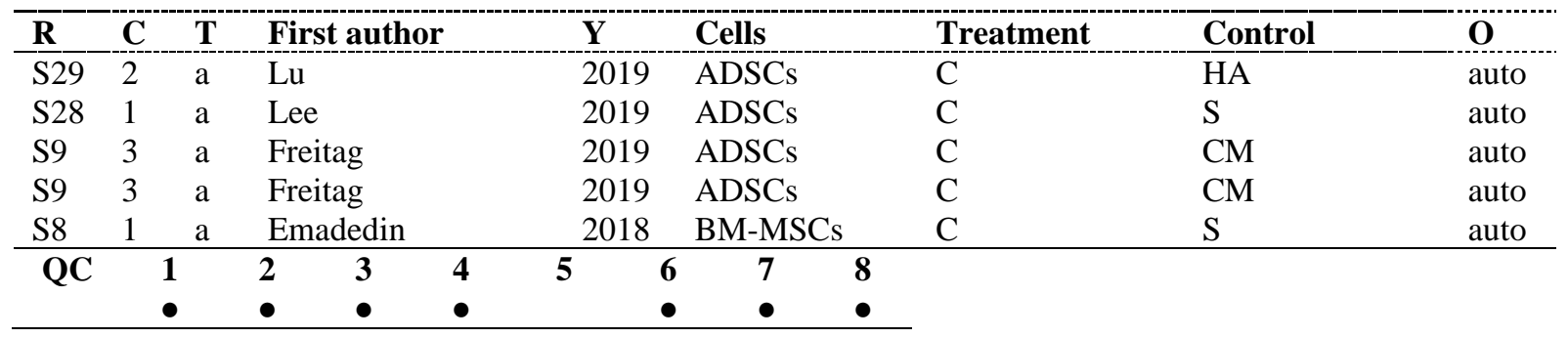


Table S17 | Details of the sub-analyses performed in a meta-analysis by Maheshwer et al. ${ }^{29}$.

Abbreviations: allo $=$ allogeneic cells; auto $=$ autologous cells; $\mathrm{C}=$ category of study as outlined in Table S2; $\mathrm{O}=$ origin of cells; $\mathrm{QC}=$ quality criteria (outlined in Table 1 in the main text; a point indicates that the corresponding quality criterion was fulfilled); $\mathrm{R}=$ reference number; $\mathrm{T}=$ type of study as outlined in Table S3; Y = year of publication. The abbreviations of the cell types and treatments are provided in Table S6.

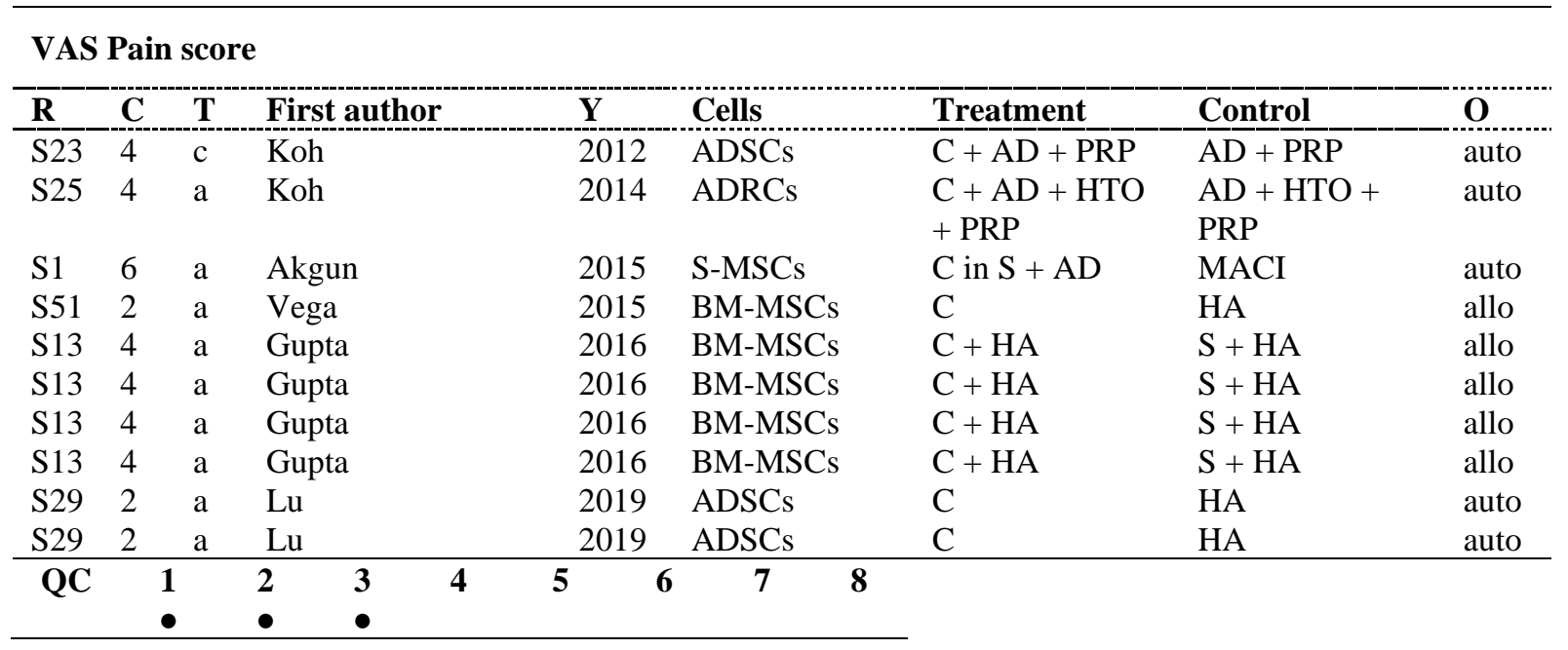

VAS Pain score after treating pkOA using stem cells

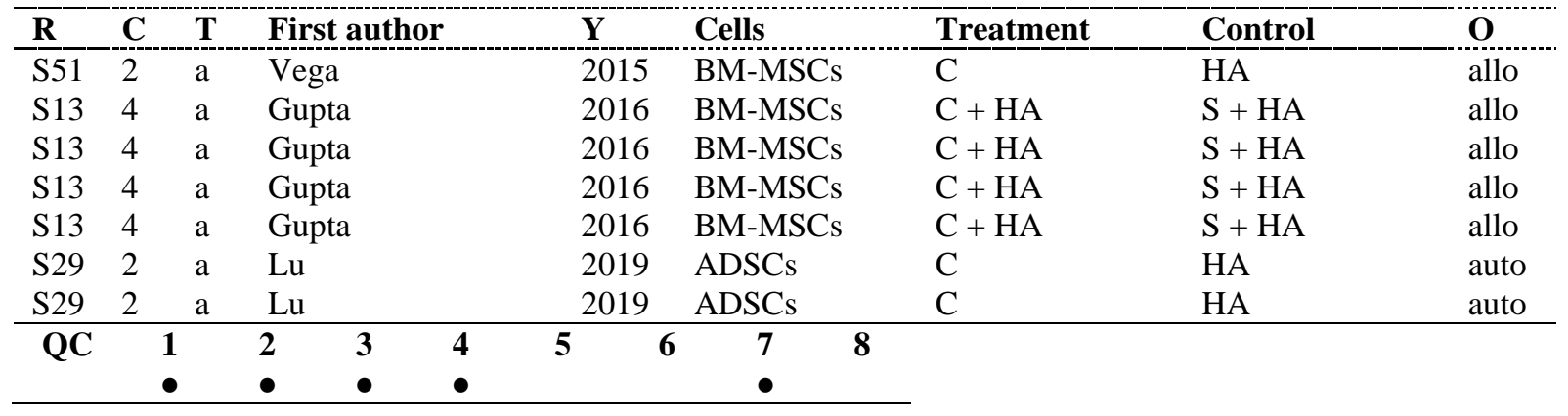

VAS Pain score after treating pkOA with stem cells in conjunction with surgery

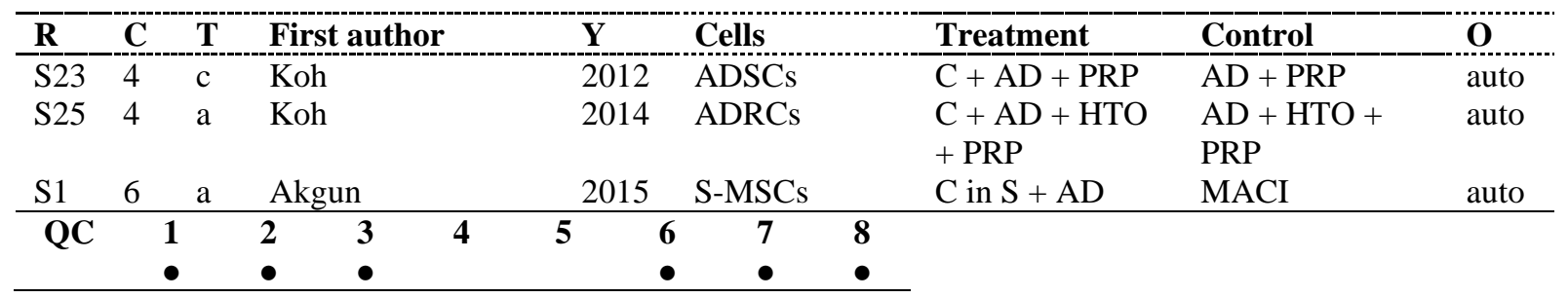




\section{WOMAC Total score}

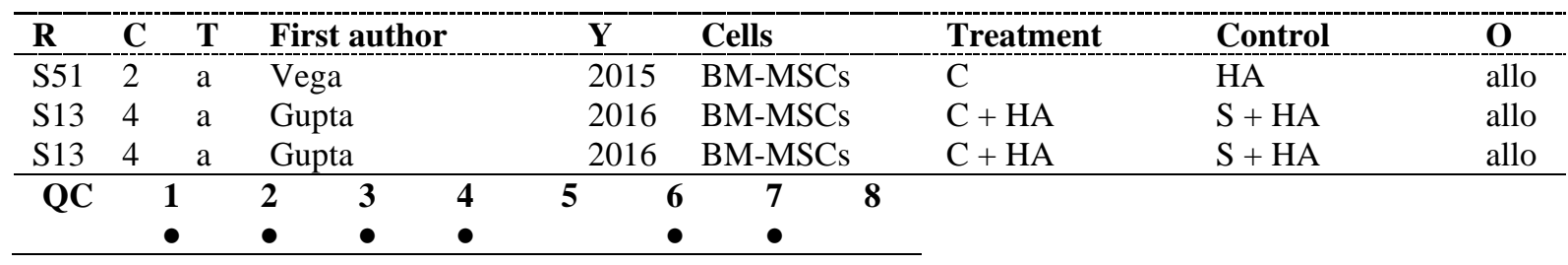

\section{IKDC scoore}

\begin{tabular}{ccccccccccccc}
\hline R & C & T & \multicolumn{2}{l}{ First author } & & Y & Cells & Treatment & Control & O \\
\hline S22 & 4 & c & Kim & & & \multicolumn{2}{c}{2018} & ADRCs & & C + AD + HTO & AD + HTO & auto \\
\hline QC & $\mathbf{1}$ & $\mathbf{2}$ & $\mathbf{3}$ & $\mathbf{4}$ & $\mathbf{5}$ & $\mathbf{6}$ & $\mathbf{7}$ & $\mathbf{8}$ & & \\
& & $\bullet$ & $\bullet$ & & $\bullet$ & $\bullet$ & $\bullet$ & $\bullet$ & & \\
\end{tabular}

\section{Lysholm score}

\begin{tabular}{|c|c|c|c|c|c|c|c|c|}
\hline $\mathbf{R}$ & C & $\mathbf{T}$ & First author & $\mathbf{Y}$ & Cells & Treatment & Control & $\mathbf{O}$ \\
\hline $\mathrm{S} 23$ & 4 & $\mathrm{c}$ & Koh & 2012 & $\mathrm{ADSCs}$ & $\mathrm{C}+\mathrm{AD}+\mathrm{PRP}$ & $\mathrm{AD}+\mathrm{PRP}$ & auto \\
\hline S25 & 4 & $\mathrm{a}$ & Koh & 2014 & ADRCs & $\begin{array}{l}\mathrm{C}+\mathrm{AD}+\mathrm{HTO} \\
+\mathrm{PRP}\end{array}$ & $\begin{array}{l}\mathrm{AD}+\mathrm{HTO}+ \\
\mathrm{PRP}\end{array}$ & auto \\
\hline
\end{tabular}

\begin{tabular}{lllllllll}
\hline QC & 1 & 2 & 3 & 4 & 5 & 6 & 7 & 8 \\
& $\bullet$ & $\bullet$ & $\bullet$ & & $\bullet$ & $\bullet$ & & $\bullet$ \\
\hline
\end{tabular}

\section{KOOS score}

\begin{tabular}{lllllllllllll}
\hline R & C & T & \multicolumn{2}{l}{ First author } & & Y & Cells & & Treatment & Control & O \\
\hline S1 & 6 & a & \multicolumn{2}{c}{ Akgun } & & 2015 & S-MSCs & & C in S + AD & MACI & auto \\
\hline QC & $\mathbf{1}$ & $\mathbf{2}$ & $\mathbf{3}$ & $\mathbf{4}$ & $\mathbf{5}$ & $\mathbf{6}$ & $\mathbf{7}$ & $\mathbf{8}$ & & \\
& & $\bullet$ & $\bullet$ & $\bullet$ & & $\bullet$ & $\bullet$ & $\bullet$ & & \\
\end{tabular}

\section{Physical function after treating pkOA using stem cells}

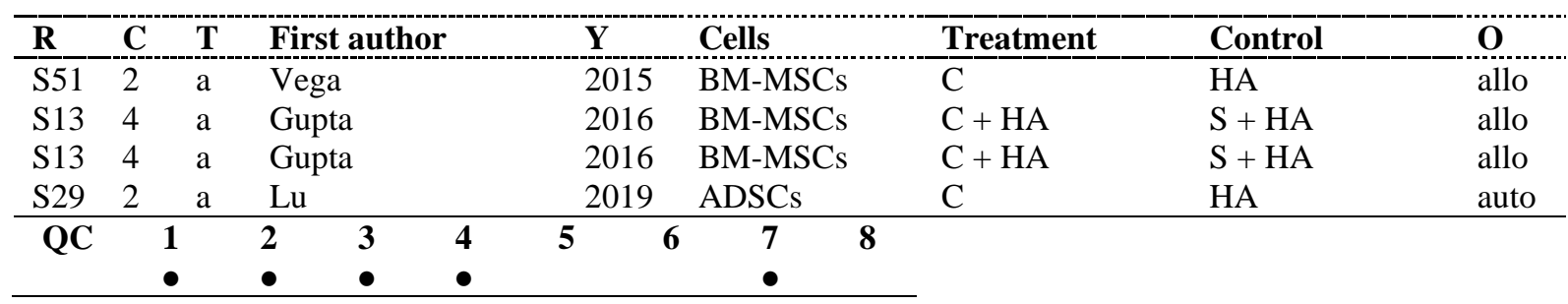


Physical function after treating pkOA with stem cells in conjunction with surgery

\begin{tabular}{lllllllll}
\hline R & C & T & First author & Y & Cells & Treatment & Control & O \\
\hline S23 & 4 & c & Koh & 2012 & ADSCs & C + AD + PRP & AD + PRP & auto \\
S1 & 6 & a & Akgun & 2015 & S-MSCs & C in S + AD & MACI & auto \\
S22 & 4 & c & Kim & 2018 & ADRCs & C + AD + HTO & AD + HTO & auto \\
S25 & 4 & a & Koh & 2014 & ADRCs & C + AD + HTO & AD + HTO + & auto \\
& & & & & PRP & \\
\hline
\end{tabular}

\begin{tabular}{lllllllll}
\hline QC & 1 & 2 & 3 & 4 & 5 & 6 & 7 & 8 \\
& $\bullet$ & $\bullet$ & $\bullet$ & & & $\bullet$ & & $\bullet$ \\
\hline
\end{tabular}

\section{Cartilage volume}

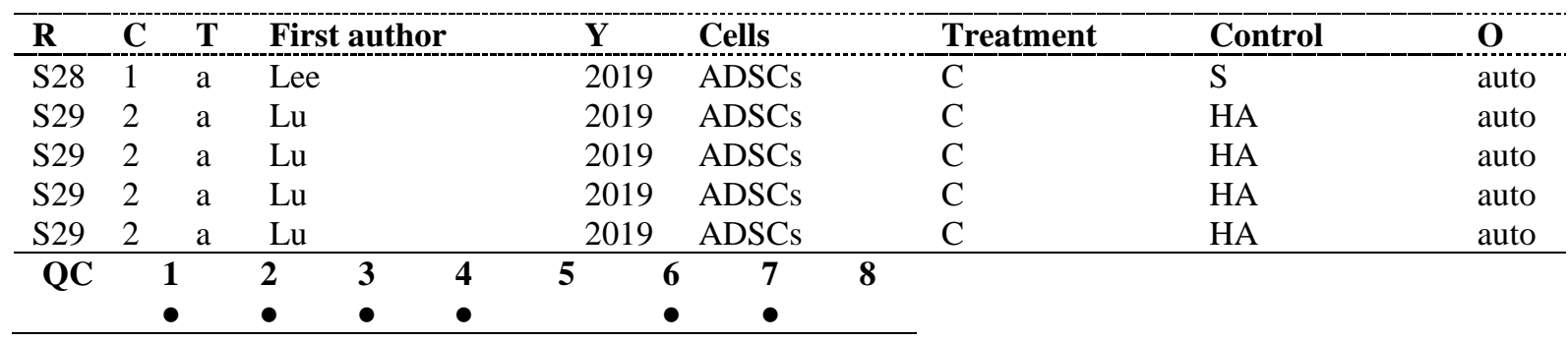

Magnetic Resonance Observation of Cartilage Repair Tissue (MOCART) score (cartilage quality)

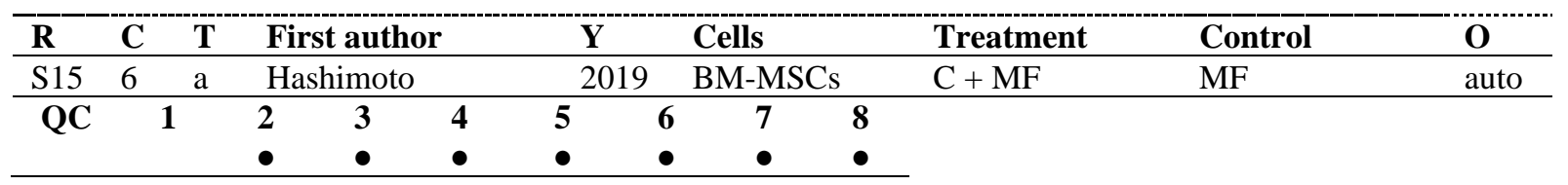

\section{Poor cartilage index}

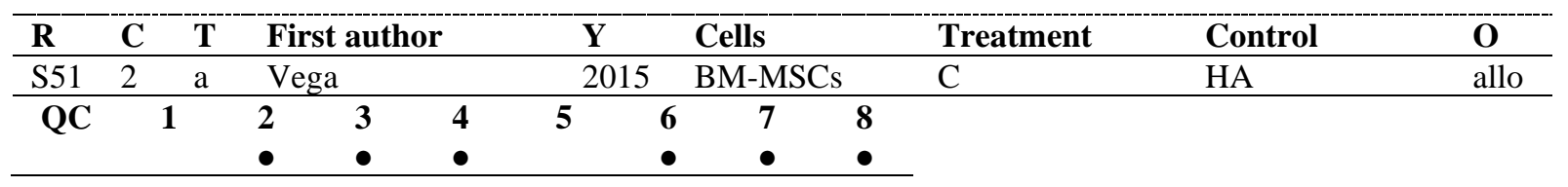

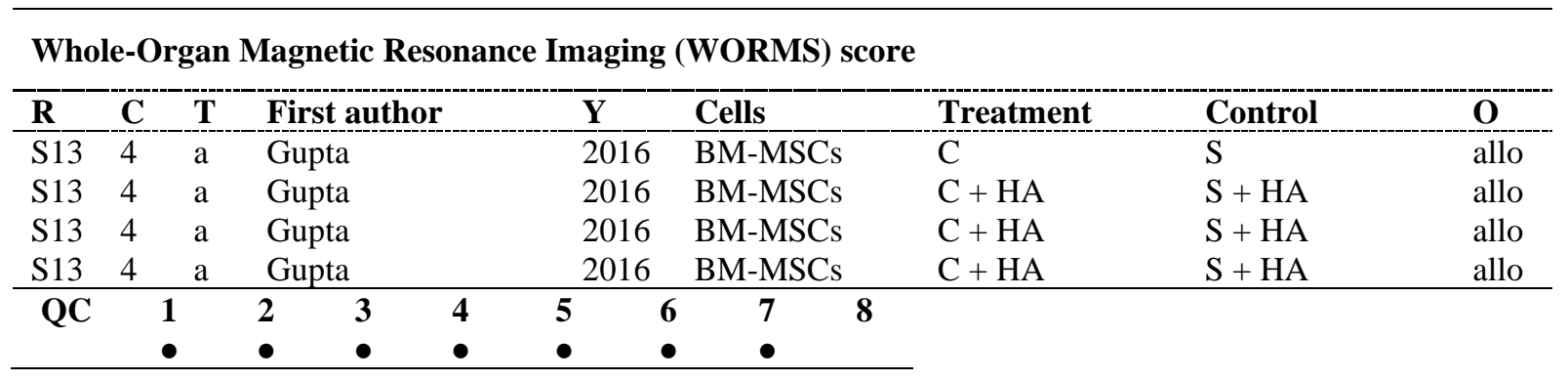


Table S18 | Details of the sub-analyses performed in a meta-analysis by Dai et al. ${ }^{24}$.

Abbreviations: allo = allogeneic cells; auto = autologous cells; $\mathrm{C}=$ category of study as outlined in Table S2; O = origin of cells; $\mathrm{QC}=$ quality criteria (outlined in Table 1 in the main text; a point indicates that the corresponding quality criterion was fulfilled); $\mathrm{R}=$ reference number; $\mathrm{T}=$ type of study as outlined in Table S3; Y = year of publication. The abbreviations of the cell types and treatments are provided in Table S6.

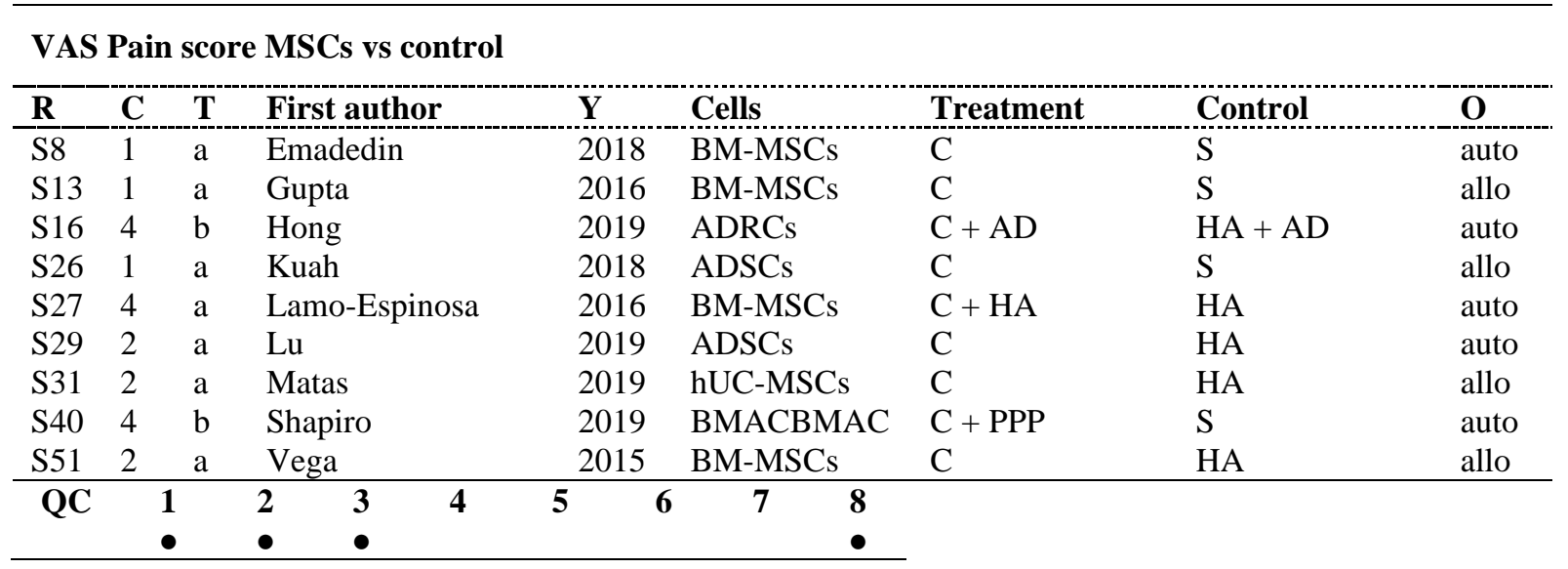

VAS Pain score MSC vs placebo

\begin{tabular}{|c|c|c|c|c|c|c|c|c|}
\hline $\mathbf{R}$ & C & $\mathbf{T}$ & First author & $\mathbf{Y}$ & Cells & Treatment & Control & O \\
\hline S8 & 1 & $\mathrm{a}$ & Emadedin & 2018 & BM-MSCs & C & $\mathrm{S}$ & auto \\
\hline S26 & 1 & $\mathrm{a}$ & Kuah & 2018 & ADSCs & $\mathrm{C}$ & $\mathrm{S}$ & allo \\
\hline S27 & 4 & $\mathrm{a}$ & Lamo-Espinosa & 2016 & BM-MSCs & $\mathrm{C}+\mathrm{HA}$ & HA & auto \\
\hline $\mathrm{S} 40$ & 4 & $\mathrm{~b}$ & Shapiro & 2019 & BMACBMAC & $\mathrm{C}+\mathrm{PPP}$ & $\mathrm{S}$ & auto \\
\hline QC & & & $\begin{array}{ll}2 & 3 \\
-\quad \\
\end{array}$ & 5 & $\begin{array}{l}8 \\
\bullet \\
\end{array}$ & & & \\
\hline
\end{tabular}

\section{VAS Pain score MSC vs HA}

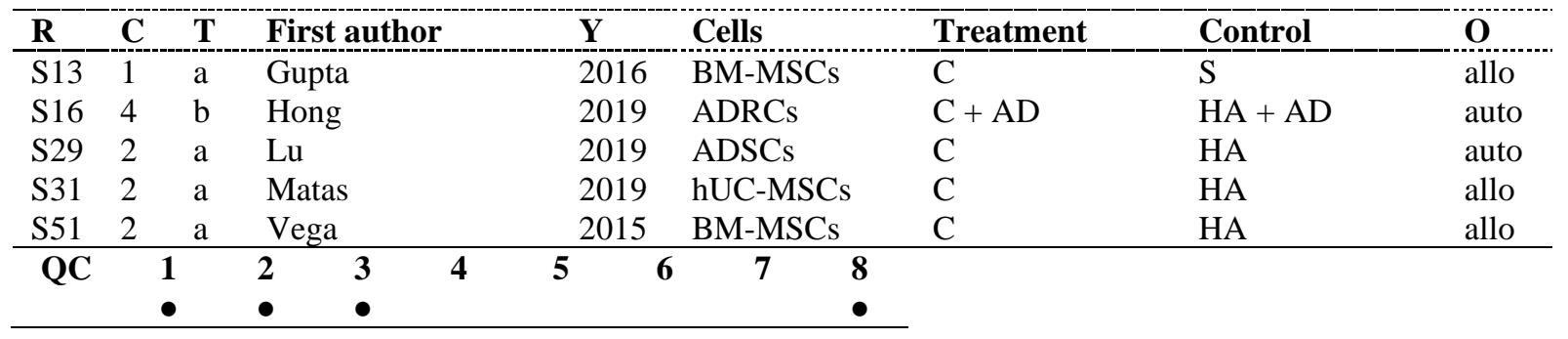




\section{WOMAC Total score MSCs vs control}

\begin{tabular}{|c|c|c|c|c|c|c|c|c|}
\hline $\mathbf{R}$ & C & $\mathbf{T}$ & First author & $\mathbf{Y}$ & Cells & Treatment & Control & $\mathbf{O}$ \\
\hline S8 & 1 & $\mathrm{a}$ & Emadedin & 2018 & BM-MSCs & $\mathrm{C}$ & $\mathrm{S}$ & auto \\
\hline S9 & 3 & $\mathrm{a}$ & Freitag & 2019 & ADSCs & $\mathrm{C}$ & $\mathrm{CM}$ & auto \\
\hline S11 & 1 & $\mathrm{a}$ & Garza & 2020 & ADRCs & $\mathrm{C}$ & $\mathrm{RS}$ & auto \\
\hline S13 & 1 & $\mathrm{a}$ & Gupta & 2016 & BM-MSCs & $\mathrm{C}$ & $\mathrm{S}$ & allo \\
\hline S27 & 4 & $\mathrm{a}$ & Lamo-Espinosa & 2016 & BM-MSCs & $\mathrm{C}+\mathrm{HA}$ & HA & auto \\
\hline S29 & 2 & $\mathrm{a}$ & $\mathrm{Lu}$ & 2019 & ADSCs & $\mathrm{C}$ & HA & auto \\
\hline S31 & 2 & $\mathrm{a}$ & Matas & 2019 & hUC-MSCs & $\mathrm{C}$ & HA & allo \\
\hline S51 & 2 & $\mathrm{a}$ & Vega & 2015 & BM-MSCs & $\mathrm{C}$ & HA & allo \\
\hline $\mathbf{Q C}$ & & & $\begin{array}{ll}2 & 3 \\
\bullet & \bullet \\
\end{array}$ & $5 \quad 6$ & 7 & & & \\
\hline
\end{tabular}

WOMAC Total score MSC vs placebo

\begin{tabular}{|c|c|c|c|c|c|c|c|c|}
\hline $\mathbf{R}$ & C & $\mathbf{T}$ & First author & $\mathbf{Y}$ & Cells & Treatment & Control & $\mathbf{O}$ \\
\hline S8 & 1 & $\mathrm{a}$ & Emadedin & 2018 & BM-MSCs & $\mathrm{C}$ & $\mathrm{S}$ & auto \\
\hline S9 & 3 & $\mathrm{a}$ & Freitag & 2019 & ADSCs & $\mathrm{C}$ & $\mathrm{CM}$ & auto \\
\hline S11 & 1 & $\mathrm{a}$ & Garza & 2020 & ADRCs & $\mathrm{C}$ & RS & auto \\
\hline S27 & 4 & $\mathrm{a}$ & Lamo-Espinosa & 2016 & BM-MSCs & $\mathrm{C}+\mathrm{HA}$ & HA & auto \\
\hline QC & & & $\begin{array}{lll}2 & 3 & 4 \\
\bullet & \bullet & \bullet \\
\end{array}$ & $\begin{array}{ll}5 \\
\bullet\end{array}$ & 7 & & & \\
\hline
\end{tabular}

\section{WOMAC Total score MSC vs HA}

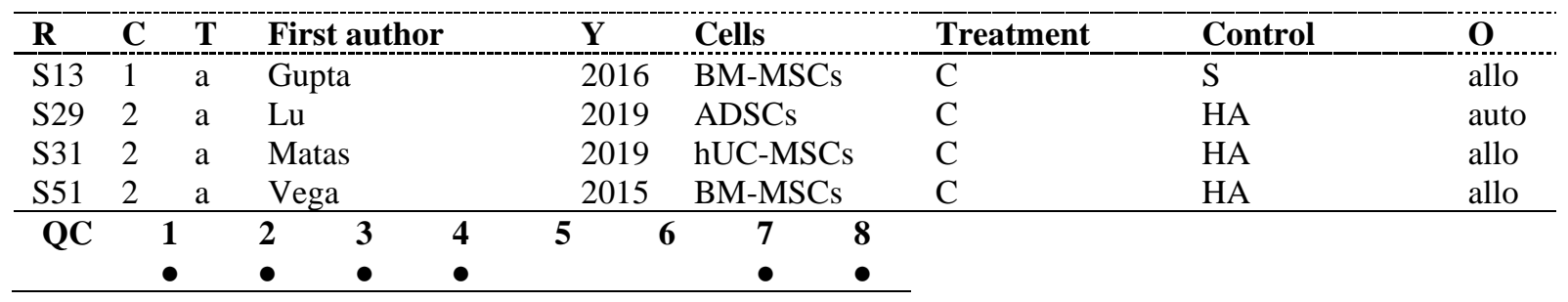

\section{VAS Pain score Adipose-derived MSCs}

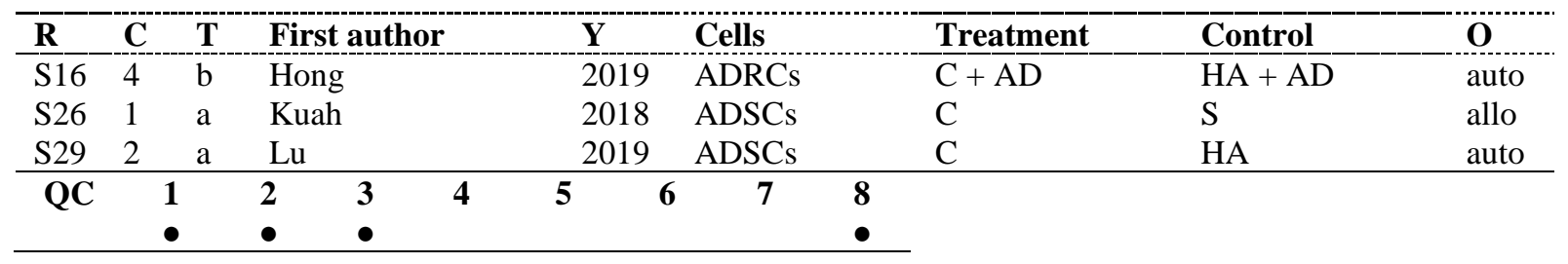


VAS Pain score Bone marrow-derived MSCs

\begin{tabular}{|c|c|c|c|c|c|c|c|c|}
\hline $\mathbf{R}$ & $\mathbf{C}$ & $\mathbf{T}$ & First author & $\mathbf{Y}$ & Cells & Treatment & Control & $\mathbf{O}$ \\
\hline S8 & 1 & $\mathrm{a}$ & Emadedin & 2018 & BM-MSCs & $\mathrm{C}$ & $\mathrm{S}$ & auto \\
\hline S13 & 1 & $\mathrm{a}$ & Gupta & 2016 & BM-MSCs & $\mathrm{C}$ & $\mathrm{S}$ & allo \\
\hline $\mathrm{S} 27$ & 4 & $\mathrm{a}$ & Lamo-Espinosa & 2016 & BM-MSCs & $\mathrm{C}+\mathrm{HA}$ & HA & auto \\
\hline $\mathrm{S} 40$ & 4 & $\mathrm{~b}$ & Shapiro & 2019 & BMACВMAC & $\mathrm{C}+\mathrm{PPP}$ & S & auto \\
\hline S51 & 2 & $\mathrm{a}$ & Vega & 2015 & BM-MSCs & $\mathrm{C}$ & HA & allo \\
\hline $\mathbf{Q C}$ & & & $\begin{array}{ll}2 & 3 \\
\bullet & \bullet \\
\end{array}$ & $5 \quad 6$ & $\begin{array}{ll}7 & 8 \\
& \bullet \\
\end{array}$ & & & \\
\hline
\end{tabular}

VAS Pain score Umbilical cord-derived MSCs

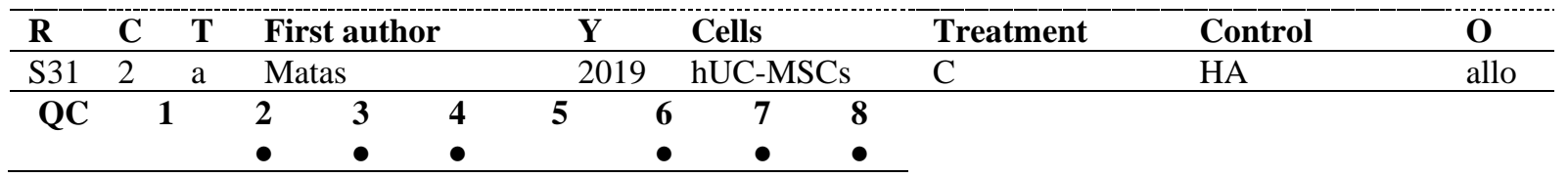

WOMAC Total score Adipose-derived MSCs

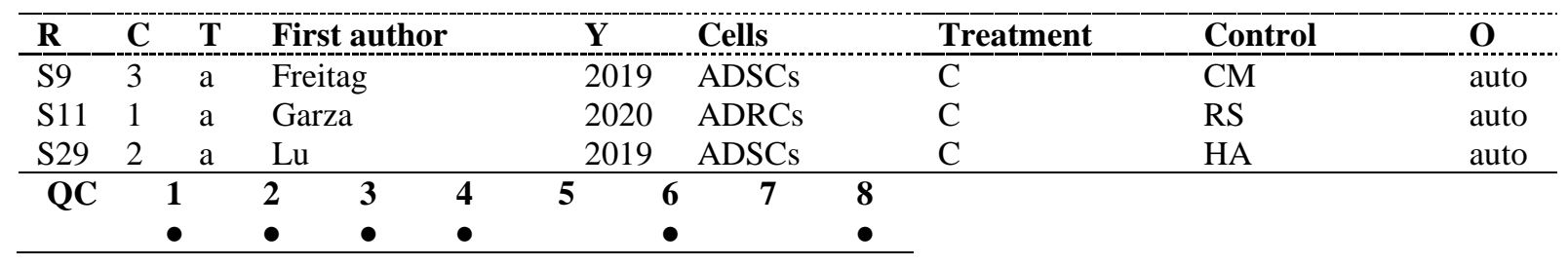

\section{WOMAC Total score Bone marrow-derived MSCs}

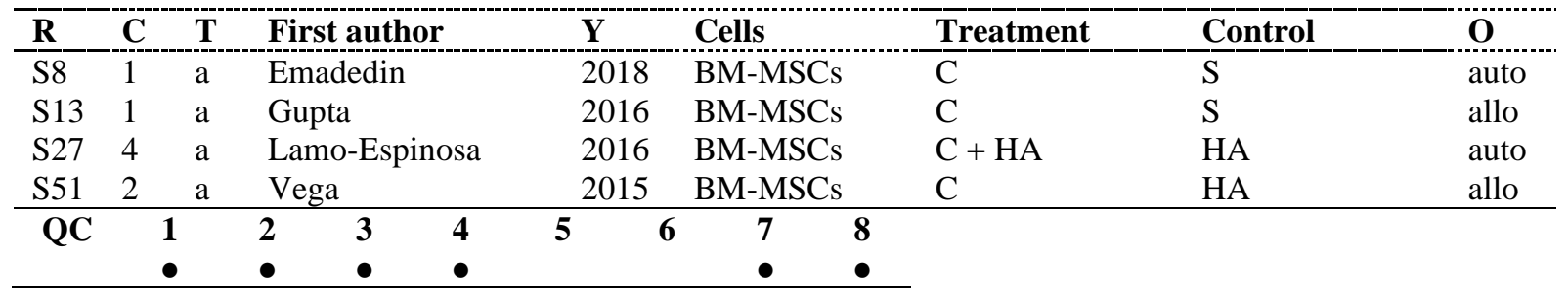

\section{WOMAC Total score Umbilical cord-derived MSCs}

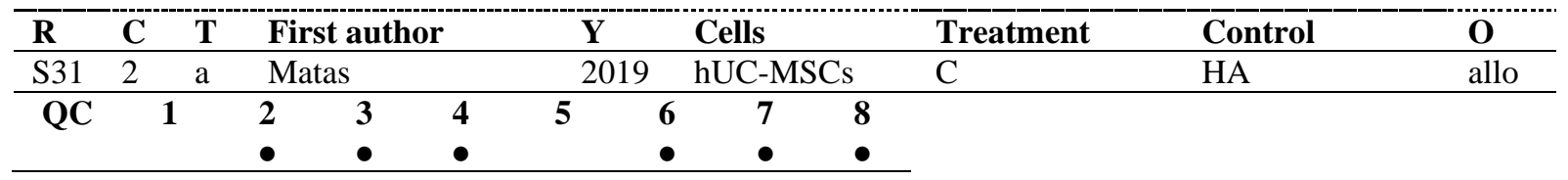




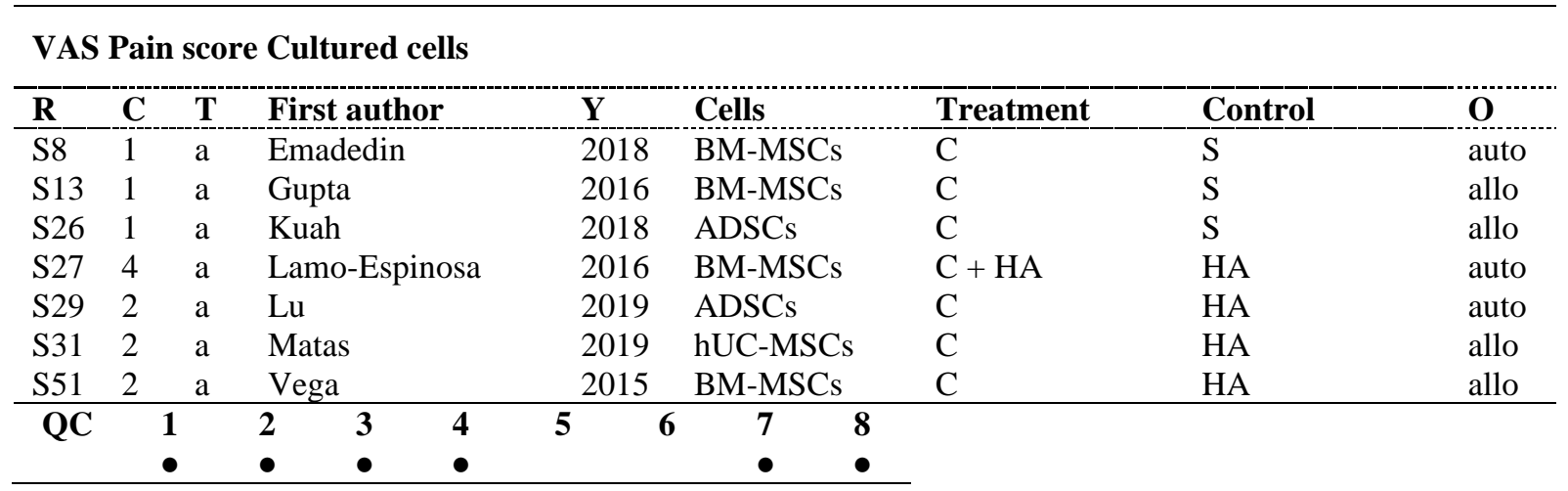

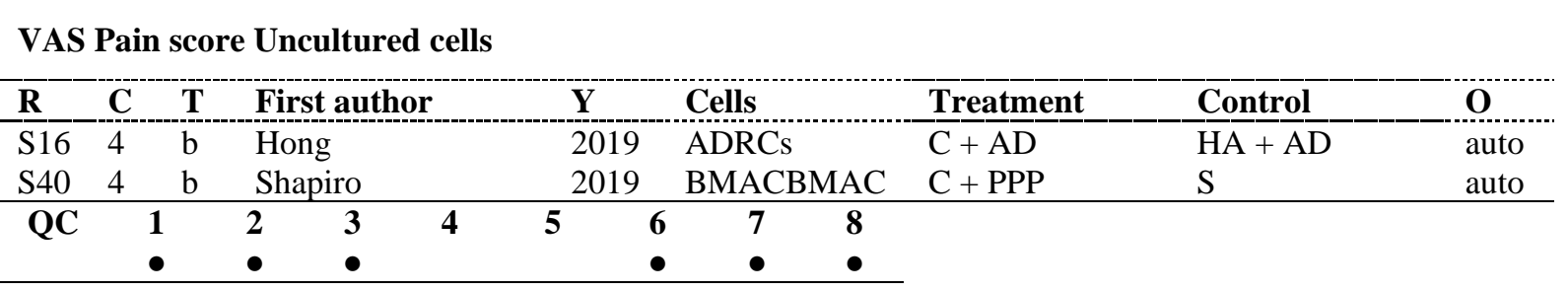

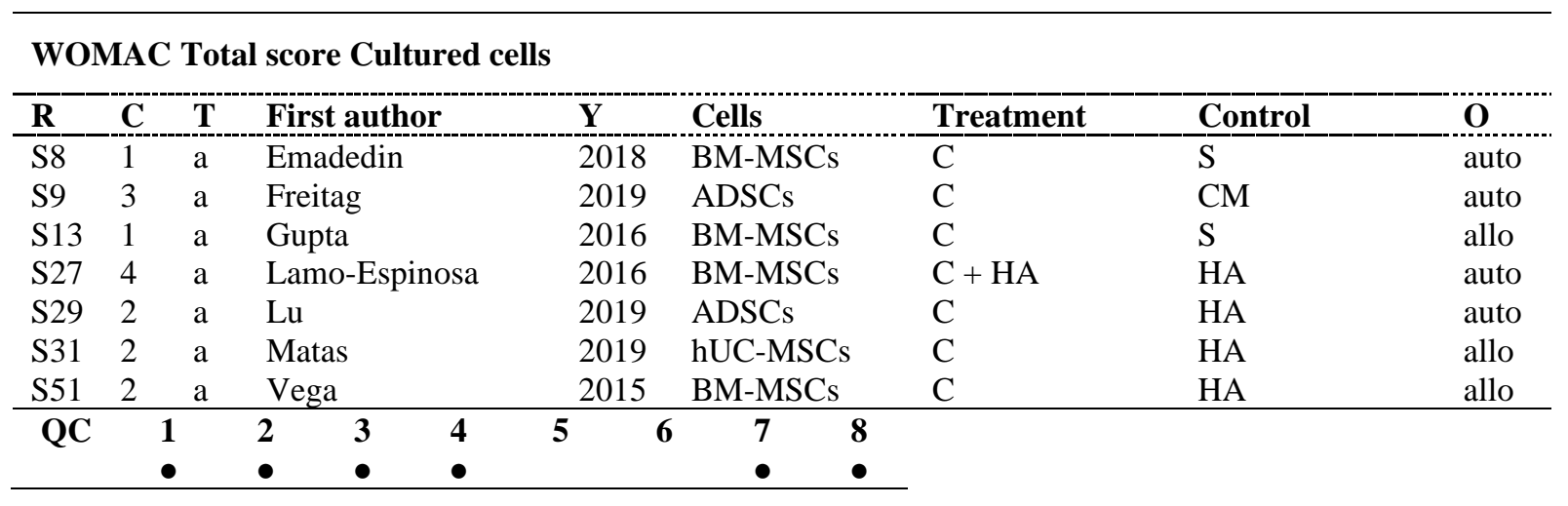

\section{WOMAC Total score Uncultured cells}

\begin{tabular}{ccccccccccccc}
\hline R & C & T & \multicolumn{2}{l}{ First author } & \multicolumn{2}{c}{ Y } & Cells & Treatment & Control & O \\
\hline S11 & 1 & a & \multicolumn{2}{l}{ Garza } & & \multicolumn{2}{c}{2020} & ADRCs & C & RS & auto \\
\hline QC & $\mathbf{1}$ & $\mathbf{2}$ & $\mathbf{3}$ & $\mathbf{4}$ & $\mathbf{5}$ & $\mathbf{6}$ & $\mathbf{7}$ & $\mathbf{8}$ & & \\
& & $\bullet$ & $\bullet$ & $\bullet$ & $\bullet$ & $\bullet$ & $\bullet$ & $\bullet$ & & \\
\hline
\end{tabular}




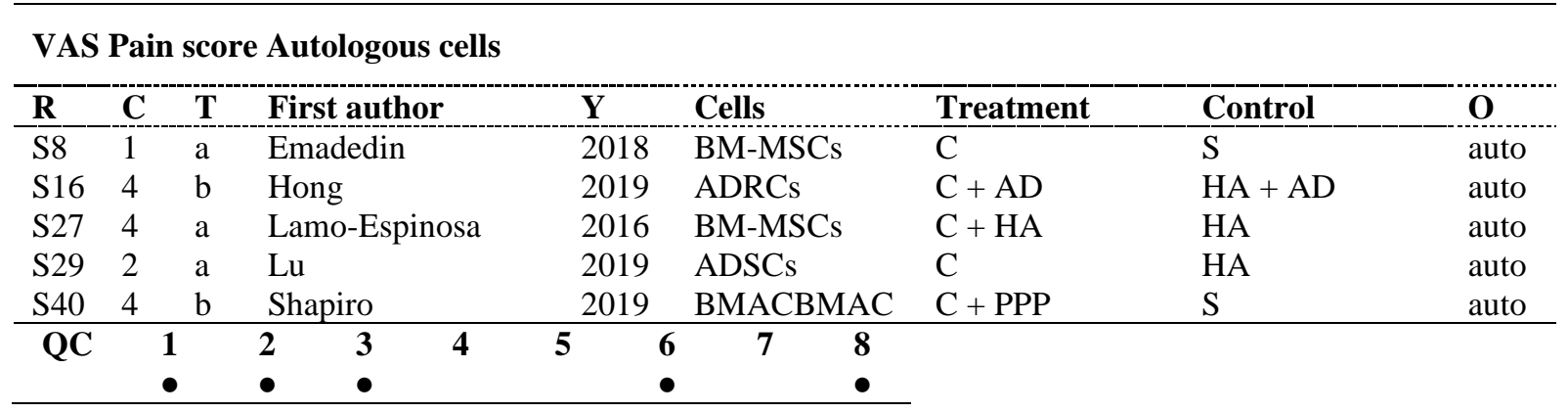

\section{VAS Pain score Allogeneic cells}

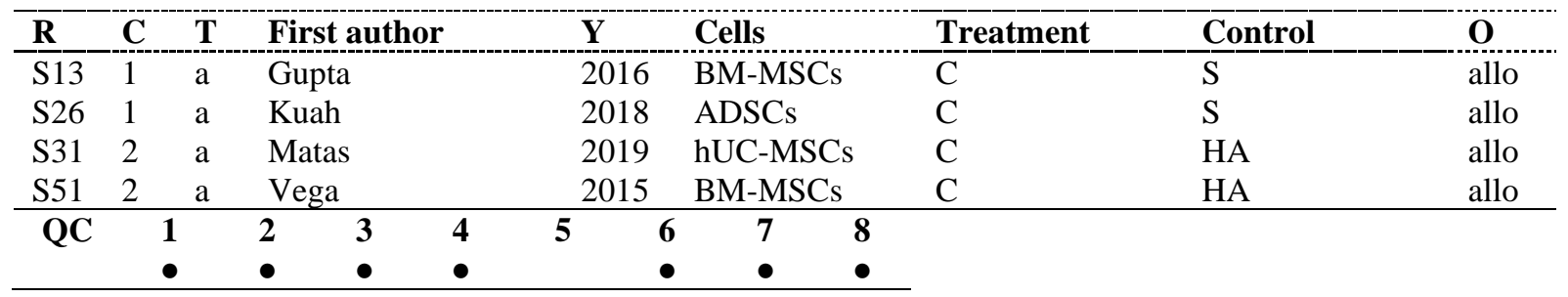

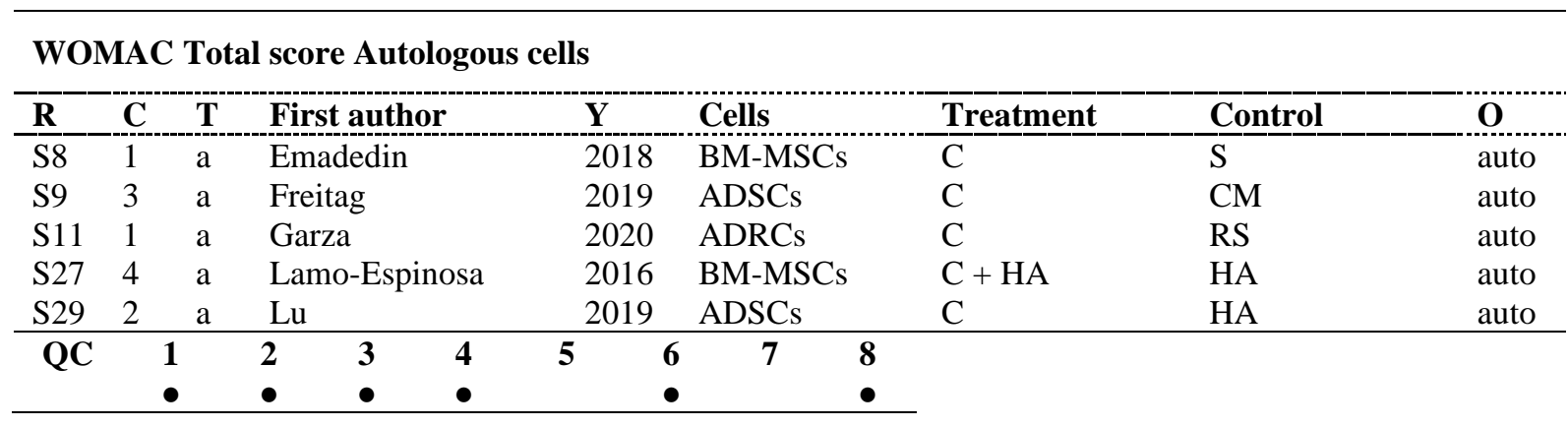

WOMAC Total score Allogeneic cells

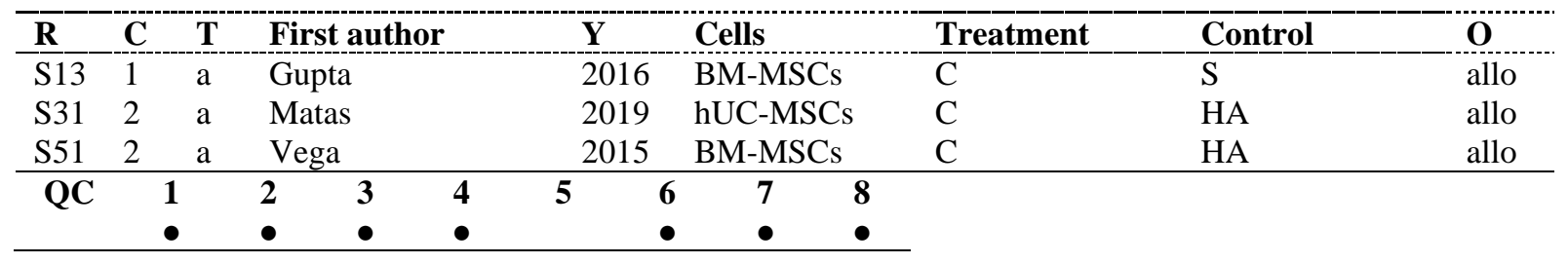


Table S19| Details of studies that were identified during an evidence-based, systematic review of the literature according to the PRISMA guidelines ${ }^{14}$ performed on August 07, 2021 in order to examine studies on treatment of primary knee osteoarthritis with stem cells.

Abbreviations: $\mathrm{AD}=$ arthroscopic debridement; ADRCs = adipose-derived regenerative cells; ADSCs = adipose-derived stem cells (obtained by culturing ADRCs); $\mathrm{AlCh}=$ allogeneic cartilage; allo = allogeneic; $\mathrm{AM}=$ arthroscopic repair of meniscus; auto = autologous; $\mathrm{AMIC}=$ autologous matrix-induced chondrogenesis; $\mathrm{AP}=$ autologous periosteum; $\mathrm{AuCh}=$ autologous chondrocytes; $\mathrm{BMA}=$ bone marrow aspirate; $\mathrm{BMAC}=$ bone marrow aspirate concentrate; BM-MSCs = bone marrow-derived mesenchymal stem cells; Ca, category of study as defined in Table S2; Ccs = subchondral application of cells; $\mathrm{Ce}=$ cell type; $\mathrm{CF}=$ centrifuged fat; $\mathrm{Ch}-\mathrm{TGF} \beta=$ chondrocytes overexpressing transforming growth factor beta; $\mathrm{CLL}=$ centrifuged liposuction liquid; $\mathrm{CM}=$ conservative management; $\mathrm{Cs}=$ chondrocytes; $\mathrm{CS}=$ corticosteroid; $\mathrm{CSCs}=\mathrm{cartilage}$ stem cells; $\mathrm{Cu}=$ culturing of cells; DFO = distal femoral osteotomy; FA = first author; FF = filtrated fat; $\mathrm{GF}=$ growth factor; HA = hayluronic acid; HTO = high tibial osteotomy; hUC-MSCs = human umbilical cord-derived MSCs; hUCB-MSCs = human umbilical cord blood-derived MSCs; MACI = matrixinduced autologous chondrocyte implant; $\mathrm{MF}=$ microfracture; $\mathrm{MFF}=$ micro-fragmented fat (from liposuction); $\mathrm{N}=$ number of patients in the treatment group; $\mathrm{O}=$ origin of cells; $\mathrm{OA}=$ oral acetaminophen; $\mathrm{pBSCs}=$ activated peripheral blood stem cells; $\mathrm{PL}=$ platelet lysate; $\mathrm{P}-\mathrm{MSCs}=$ placental MSCs; PRP = platelet rich plasma; RS = Ringer solution; $\mathrm{S}=$ scaffold; $\mathrm{Sa}=$ saline; $\mathrm{S}-\mathrm{MSCs}=$ matrix-induced MSCs from synovia; $\mathrm{SR}=$ surgical repair; $\mathrm{R}=$ reference; $\mathrm{T}=$ type of study as defined in Table S3; TPA = total knee arthroplasty (internal control); $\mathrm{Y}=\mathrm{year}$ of publication;

Remark: a, in a study of Centeno et al. (2021) a combination of autologous BMAC + PRP + PL was administered into the knee joint and the subchondral bone (treatment group), or into the knee joint alone (control group), respectively.

\begin{tabular}{|c|c|c|c|c|c|c|c|c|c|c|}
\hline $\mathbf{R}$ & $\mathbf{C a}$ & $\mathbf{T}$ & FA & $\mathbf{Y}$ & $\mathrm{Ce}$ & $\mathbf{O}$ & Treatment & $\mathbf{N}$ & Control & $\mathrm{Cu}$ \\
\hline S7 & $\mathrm{I}$ & $\mathrm{a}$ & Cherian & 2015 & Ch-TGFß & allo & $\mathrm{C}$ & 50 & $\mathrm{Sa}$ & Yes \\
\hline S13 & I & $\mathrm{a}$ & Gupta & 2016 & BM-MSCs & allo & $\mathrm{C}+\mathrm{HA}$ & 40 & $\mathrm{C}$ or $\mathrm{S}$ & Yes \\
\hline S8 & I & $\mathrm{a}$ & Emadedin & 2018 & BM-MSCs & auto & $\mathrm{C}$ & 19 & $\mathrm{Sa}$ & Yes \\
\hline $\mathrm{S} 22$ & I & $\mathrm{a}$ & Kim & 2018 & Ch-TGFß & allo & $\mathrm{C}$ & 78 & $\mathrm{Sa}$ & Yes \\
\hline S26 & I & $\mathrm{a}$ & Kuah & 2018 & ADSCs & allo & $\mathrm{C}$ & 16 & $\mathrm{Sa}$ & Yes \\
\hline $\mathrm{S} 21$ & I & $\mathrm{a}$ & Khalifeh Soltani & 2019 & P-MSCs & allo & $\mathrm{C}$ & 10 & $\mathrm{Sa}$ & Yes \\
\hline S28 & I & $\mathrm{a}$ & Lee & 2019 & ADSCs & auto & $\mathrm{C}$ & 12 & $\mathrm{Sa}$ & Yes \\
\hline S11 & $\mathrm{I}$ & $\mathrm{a}$ & Garza & 2020 & ADRCs & auto & $\mathrm{C}$ & 26 & $\mathrm{RS}$ & No \\
\hline S51 & II & $\mathrm{a}$ & Vega & 2015 & BM-MSCs & allo & $\mathrm{C}$ & 15 & $\mathrm{HA}$ & Yes \\
\hline S52 & II & $\mathrm{a}$ & Wang & 2016 & hUC-MSCs & allo & $\mathrm{C}$ & 18 & $\mathrm{HA}$ & Yes \\
\hline $\mathrm{S} 12$ & II & $\mathrm{a}$ & Goncars & 2017 & BMAC & auto & $\mathrm{C}$ & 28 & $\mathrm{HA}$ & No \\
\hline S29 & II & $\mathrm{a}$ & $\mathrm{Lu}$ & 2019 & ADSCs & auto & $\mathrm{C}$ & 26 & HA & Yes \\
\hline S31 & II & $\mathrm{a}$ & Matas & 2019 & hUC-MSCs & allo & $\mathrm{C}$ & 18 & HA & Yes \\
\hline S3 & II & $\mathrm{a}$ & Anz & 2020 & BMAC & auto & $\mathrm{C}$ & 45 & PRP & No \\
\hline S80 & II & a & Dallo & 2021 & MFF & auto & $\mathrm{C}$ & 40 & $\mathrm{PRP}+\mathrm{HA}$ & No \\
\hline $\mathrm{S} 121$ & II & $\mathrm{d}$ & $\mathrm{Kim}$ & 2020 & ADRCs & auto & $\mathrm{C}$ & 30 & $\mathrm{HA}$ & No \\
\hline
\end{tabular}


Table S19 (cont.)

\begin{tabular}{|c|c|c|c|c|c|c|c|c|c|c|}
\hline $\mathbf{R}$ & $\mathbf{C a}$ & $\mathbf{T}$ & FA & $\mathbf{Y}$ & $\mathrm{Ce}$ & $\mathbf{O}$ & Treatment & $\mathbf{N}$ & Control & $\mathbf{C u}$ \\
\hline S10 & III & $\mathrm{a}$ & Garay-Mendoza & 2018 & BMAC & auto & $\mathrm{C}$ & 61 & $\mathrm{OA}$ & No \\
\hline S44 & III & $\mathrm{a}$ & Song & 2018 & ADSCs & auto & $\mathrm{C}$ & 18 & $\mathrm{C}$ & Yes \\
\hline S9 & III & $\mathrm{a}$ & Freitag & 2019 & ADSCs & auto & $\mathrm{C}$ & 20 & $\mathrm{CM}$ & Yes \\
\hline S187 & III & $\mathrm{a}$ & Zhao & 2019 & ADSCs & allo & $\mathrm{C}$ & 18 & $\mathrm{C}$ & Yes \\
\hline S4 & III & $\mathrm{a}$ & Bastos & 2020 & BM-MSCs & auto & $\mathrm{C}$ & 16 & $\mathrm{C}+\mathrm{PRP}+\mathrm{CS}$ & Yes \\
\hline $\mathrm{S} 135$ & III & $\mathrm{a}$ & $\mathrm{Lu}$ & 2020 & ADSCs & allo & $\mathrm{C}$ & 22 & $\mathrm{C}$ & Yes \\
\hline $\mathrm{S} 105$ & III & $\mathrm{b}$ & Hernigou & 2018 & $\mathrm{BMAC}$ & auto & $\mathrm{C}$ & 30 & $\mathrm{TPA}$ & No \\
\hline S106 & III & $\mathrm{b}$ & Hernigou & 2020 & BMAC & auto & $\mathrm{C}$ & 60 & $\mathrm{Csc}$ & No \\
\hline S107 & III & $b$ & Hernigou & 2020 & BMAC & auto & C & 140 & TPA & No \\
\hline $\mathrm{S} 20$ & III & $\mathrm{c}$ & Jo & 2014 & $\mathrm{ADSCs}$ & auto & $\mathrm{C}$ & 18 & $\mathrm{C}$ & Yes \\
\hline S36 & III & $\mathrm{c}$ & Pers & 2016 & ADSCs & auto & $\mathrm{C}$ & 18 & $\mathrm{C}$ & Yes \\
\hline S19 & III & $\mathrm{c}$ & Jo & 2017 & ADSCs & auto & $\mathrm{C}$ & 18 & $\mathrm{C}$ & Yes \\
\hline S35 & III & $\mathrm{c}$ & Pers & 2018 & ADSCs & auto & $\mathrm{C}$ & 18 & $\mathrm{C}$ & Yes \\
\hline S76 & III & $\mathrm{c}$ & Chahal & 2019 & ADSCs & auto & $\mathrm{C}$ & 12 & $\mathrm{C}$ & Yes \\
\hline S89 & III & $\mathrm{c}$ & Estrada & 2020 & ADRCs & auto & $\mathrm{C}$ & 33 & $\mathrm{BMAC}+\mathrm{PRP}$ & No \\
\hline $\mathrm{S} 112$ & III & $\mathrm{c}$ & Kazemian & 2020 & BM-MSCs & auto & $\mathrm{C}$ & 20 & Not described & Yes \\
\hline $\mathrm{S} 147$ & III & $\mathrm{c}$ & Papalia & 2020 & MFF & auto & $\mathrm{C}$ & 8 & $\mathrm{CF}$ or $\mathrm{FF}$ & No \\
\hline S60 & III & c & Bistolfi & 2021 & MFF & auto & $\mathrm{C}$ & 27 & $\mathrm{CF}$ & No \\
\hline S32 & III & d & Mautner & 2019 & $\mathrm{MFF}$ & auto & $\mathrm{C}$ & 23 & BMAC & No \\
\hline S54 & III & $\mathrm{d}$ & Yokota & 2019 & ADRCs & auto & $\mathrm{C}$ & 38 & $\mathrm{C}$ & No \\
\hline $\mathrm{S} 132$ & III & $\mathrm{d}$ & $\mathrm{Li}$ & 2020 & BM-MSCs & auto & $\mathrm{C}$ & 40 & $\mathrm{AD}+\mathrm{HA}$ & Yes \\
\hline $\mathrm{S} 168$ & III & $\mathrm{d}$ & Simunec & 2020 & ADRCs & auto & $\mathrm{C}$ & 6 & $\mathrm{C}+\mathrm{PRP}$ & No \\
\hline S50 & IV & $\mathrm{a}$ & Varma & 2010 & BM-MSCs & auto & $\mathrm{C}+\mathrm{AD}$ & 25 & $\mathrm{AD}$ & Yes \\
\hline S53 & IV & $\mathrm{a}$ & Wong & 2013 & BM-MSCs & auto & $\mathrm{C}+\mathrm{MF}+\mathrm{HTO}+\mathrm{HA}$ & 28 & $\mathrm{HA}+\mathrm{HTO}+\mathrm{MF}$ & Yes \\
\hline S25 & IV & $\mathrm{a}$ & Koh & 2014 & ADRCs & auto & $\mathrm{C}+\mathrm{AD}+\mathrm{HTO}+\mathrm{PRP}$ & 21 & $\mathrm{PRP}+\mathrm{AD}+\mathrm{HTO}$ & No \\
\hline S27 & IV & $\mathrm{a}$ & Lamo-Espinosa & 2016 & BM-MSCs & auto & $\mathrm{C}+\mathrm{HA}$ & 10 & $\mathrm{HA}$ & Yes \\
\hline S177 & IV & $\mathrm{a}$ & Turajane & 2017 & pBSCs & auto & $\mathrm{C}+\mathrm{AD}+\mathrm{MF}$ & 20 & HA & No \\
\hline S5 & IV & $\mathrm{a}$ & Bastos & 2018 & BM-MSCs & auto & $\mathrm{C}+\mathrm{PRP}$ & 9 & $\mathrm{C}$ & Yes \\
\hline S56 & IV & $\mathrm{a}$ & Zhang & 2018 & ADSCs & auto & $\mathrm{C}+\mathrm{HA}$ & 36 & $\mathrm{C}$ & Yes \\
\hline S128 & IV & $\mathrm{a}$ & Lamo-Espinosa & 2018 & BM-MSCs & auto & $\mathrm{C}+\mathrm{HA}$ & 10 & HA & Yes \\
\hline S149 & IV & $\mathrm{a}$ & Peretti & 2018 & MFF & auto & $\mathrm{C}+\mathrm{AD}$ & 20 & $\mathrm{AD}$ & No \\
\hline S129 & IV & $\mathrm{a}$ & Lamo-Espinosa & 2020 & BM-MSCs & auto & $\mathrm{C}+\mathrm{PRP}$ & 30 & PRP & Yes \\
\hline $\mathrm{S} 152$ & IV & $\mathrm{a}$ & Qiao & 2020 & ADSCs & auto & $\mathrm{C}+\mathrm{AD}+\mathrm{MF}+\mathrm{HA}$ & 10 & $\mathrm{MF}$ or $\mathrm{MF}+\mathrm{HA}$ & Yes \\
\hline $\mathrm{S} 154$ & IV & a & Ruane & 2021 & BMAC & auto & $\mathrm{C}+\mathrm{PRP}$ & 17 & $\mathrm{HA}$ & No \\
\hline $\mathrm{S} 41$ & IV & $\mathrm{b}$ & Shapiro & 2017 & BMAC & auto & $\mathrm{C}+\mathrm{PPP}$ & 25 & $\mathrm{~S}$ & No \\
\hline S16 & IV & $\mathrm{b}$ & Hong & 2019 & ADRCs & auto & $\mathrm{C}+\mathrm{AD}$ & 16 & $\mathrm{HA}+\mathrm{AD}$ & No \\
\hline $\mathrm{S} 40$ & IV & $\mathrm{b}$ & Shapiro & 2019 & BMAC & auto & $\mathrm{C}+\mathrm{PPP}$ & 25 & $\mathrm{~S}$ & No \\
\hline
\end{tabular}


Table S19 (cont.)

\begin{tabular}{|c|c|c|c|c|c|c|c|c|c|c|}
\hline $\mathbf{R}$ & $\mathbf{C a}$ & $\mathbf{T}$ & FA & $\mathbf{Y}$ & $\mathrm{Ce}$ & $\mathbf{O}$ & Treatment & $\mathbf{N}$ & Control & $\mathbf{C u}$ \\
\hline $\mathrm{S} 23$ & IV & $\mathrm{c}$ & Koh & 2012 & ADSCs & auto & $\mathrm{C}+\mathrm{AD}+\mathrm{PRP}$ & 25 & $A D+P R P$ & $?$ \\
\hline S119 & IV & $\mathrm{c}$ & Kim & 2015 & ADRCs & auto & $\mathrm{C}+\mathrm{AD}+\mathrm{PRP}$ & 20 & $\mathrm{C}$ in $\mathrm{S}+\mathrm{AD}$ & No \\
\hline S33 & IV & $\mathrm{c}$ & Nguyen & 2017 & ADRCs & auto & $\mathrm{C}+\mathrm{AD}+\mathrm{MF}+\mathrm{PRP}$ & 15 & $\mathrm{AD}+\mathrm{MF}$ & No \\
\hline S118 & IV & $\mathrm{c}$ & Kim & 2018 & ADRCs & auto & $\mathrm{C}+\mathrm{AD}+\mathrm{HTO}$ & 50 & HTO & No \\
\hline S47 & IV & $\mathrm{c}$ & Tran & 2019 & ADRCs & auto & $\mathrm{C}+\mathrm{AD}+\mathrm{MF}$ & 18 & $\mathrm{AD}+\mathrm{MF}$ & No \\
\hline S86 & IV & c & Ehlers & 2020 & ADRCs & auto & $\mathrm{C}+\mathrm{PRP}$ & 8 & PRP & No \\
\hline $\mathrm{S} 72$ & IV & $\mathrm{d}$ & Centeno & 2014 & BMAC & auto & $\mathrm{C}+$ adipose graft & 840 & $\mathrm{C}$ & No \\
\hline $\mathrm{S} 116$ & IV & $\mathrm{d}$ & Kim & 2015 & ADRCs & auto & $\mathrm{C}$ in $\mathrm{S}+\mathrm{AD}$ & 17 & $\mathrm{C}+\mathrm{AD}$ & No \\
\hline S175 & IV & $\mathrm{d}$ & Srinivas & 2015 & BMAC & auto & $\mathrm{C}+\mathrm{PRP}+\mathrm{Co}$ & 65 & $\mathrm{Co}$ & No \\
\hline $\mathrm{S} 130$ & IV & $\mathrm{d}$ & Lee & 2021 & BMAC & auto & $\mathrm{C}+\mathrm{MF}+\mathrm{HTO}$ & 74 & hUCB-MSCs & No \\
\hline S71 & IV & $\mathrm{d}$ & Centeno & 2021 & BMAC & auto & $\mathrm{BMAC}+\mathrm{PRP}+\mathrm{PL}^{\mathrm{a}}$ & 80 & $\mathrm{BMAC}+\mathrm{PRP}+\mathrm{PL}^{\mathrm{a}}$ & \\
\hline $\mathrm{S} 136$ & IV & $\mathrm{d}$ & Magnanelli & 2021 & MFF & auto & $\mathrm{C}+\mathrm{HTO}$ & 42 & HTO & No \\
\hline S81 & $\mathrm{V}$ & $\mathrm{e}$ & Davatchi & 2011 & BM-MSCs & auto & $\mathrm{C}$ & 4 & None & Yes \\
\hline S146 & $\mathrm{V}$ & $\mathrm{e}$ & Pak & 2011 & ADRCs & auto & $\mathrm{C}+\mathrm{PRP}+\mathrm{HA}$ & 2 & None & No \\
\hline S87 & $\mathrm{V}$ & $\mathrm{e}$ & Emadedin & 2012 & BM-MSCs & auto & $\mathrm{C}$ & 6 & None & Yes \\
\hline $\mathrm{S} 102$ & $\mathrm{~V}$ & $\mathrm{e}$ & Hauser & 2013 & BMA & auto & $\mathrm{C}$ & 3 & None & No \\
\hline S24 & $\mathrm{V}$ & $\mathrm{e}$ & Koh & 2013 & ADRCs & auto & $\mathrm{C}+\mathrm{PRP}+\mathrm{HA}$ & 33 & None & No \\
\hline S34 & $\mathrm{V}$ & $\mathrm{e}$ & Orozco & 2013 & BM-MSCs & auto & $\mathrm{C}$ & 12 & None & Yes \\
\hline S178 & $\mathrm{V}$ & $\mathrm{e}$ & Turajane & 2013 & pBSCs & auto & $\mathrm{C}+\mathrm{MF}+\mathrm{GF}+\mathrm{HA}$ & 4 & None & No \\
\hline S58 & $\mathrm{V}$ & $\mathrm{e}$ & Ahmad & 2014 & pBSCs & auto & $\mathrm{C}$ & 10 & None & No \\
\hline S66 & $\mathrm{V}$ & $\mathrm{e}$ & Bui & 2014 & ADRCs & auto & $\mathrm{C}+\mathrm{PRP}$ & 23 & None & No \\
\hline S123 & $\mathrm{V}$ & $\mathrm{e}$ & Koh & 2014 & ADRCs & auto & $\mathrm{C}+\mathrm{AD}$ & 37 & None & No \\
\hline S144 & $\mathrm{V}$ & $\mathrm{e}$ & Orozco & 2014 & BM-MSCs & auto & $\mathrm{C}$ & 12 & None & Yes \\
\hline S73 & $\mathrm{V}$ & $\mathrm{e}$ & Centeno & 2015 & BMAC & auto & $\mathrm{C}$ & 373 & None & No \\
\hline S88 & $\mathrm{V}$ & $\mathrm{e}$ & Emadedin & 2015 & BM-MSCs & auto & $\mathrm{C}$ & 6 & None & Yes \\
\hline S96 & $\mathrm{V}$ & $\mathrm{e}$ & Gibbs & 2015 & ADRCs & auto & $\mathrm{C}+\mathrm{PRP}$ & 4 & None & No \\
\hline S114 & $\mathrm{V}$ & $\mathrm{e}$ & Kim & 2015 & ADRCs & auto & $\mathrm{C}$ in $\mathrm{S}+\mathrm{AD}$ & 55 & None & No \\
\hline $\mathrm{S} 124$ & $\mathrm{~V}$ & $\mathrm{e}$ & Koh & 2015 & ADRCs & auto & $\mathrm{C}+\mathrm{AL}$ & 30 & None & No \\
\hline S82 & $\mathrm{V}$ & $\mathrm{e}$ & Davatchi & 2016 & BM-MSCs & auto & $\mathrm{C}$ & 4 & None & Yes \\
\hline S90 & $\mathrm{V}$ & $\mathrm{e}$ & Fodor & 2016 & ADRCs & auto & $\mathrm{C}$ & 6 & None & No \\
\hline S115 & $\mathrm{V}$ & $\mathrm{e}$ & Kim & 2016 & ADRCs & auto & $\mathrm{C}$ in $\mathrm{S}+\mathrm{AD}$ & 20 & None & No \\
\hline $\mathrm{S} 145$ & $\mathrm{~V}$ & $\mathrm{e}$ & Pak & 2016 & ADRCs & auto & $\mathrm{C}+\mathrm{PRP}+\mathrm{HA}$ & 3 & None & No \\
\hline S159 & $\mathrm{V}$ & $\mathrm{e}$ & Sampson & 2016 & BMAC & auto & $\mathrm{C}+\mathrm{PRP}$ & 125 & None & No \\
\hline $\mathrm{S} 43$ & $\mathrm{~V}$ & $\mathrm{e}$ & Soler & 2016 & BM-MSCs & auto & $\mathrm{C}$ & 15 & None & Yes \\
\hline $\mathrm{S} 2$ & $\mathrm{~V}$ & $\mathrm{e}$ & Al-Najar & 2017 & BM-MSCs & auto & $\mathrm{C}$ & 13 & None & Yes \\
\hline S18 & $\mathrm{V}$ & e & Hudetz & 2017 & MFF & auto & $\mathrm{C}$ & 17 & None & No \\
\hline $\mathrm{S} 148$ & $\mathrm{~V}$ & $\mathrm{e}$ & Park & 2017 & hUCB-MSCs & allo & $\mathrm{C}+\mathrm{MF}$ & 6 & None & Yes \\
\hline $\mathrm{S} 150$ & $\mathrm{~V}$ & $\mathrm{e}$ & Pintat & 2017 & ADRCs & auto & $\mathrm{C}+\mathrm{PRP}$ & 19 & None & $?$ \\
\hline
\end{tabular}




\section{Table S19 (cont.)}

\begin{tabular}{|c|c|c|c|c|c|c|c|c|c|c|}
\hline $\mathbf{R}$ & $\mathbf{C a}$ & $\mathbf{T}$ & FA & $\mathbf{Y}$ & $\mathrm{Ce}$ & $\mathbf{O}$ & Treatment & $\mathbf{N}$ & Control & $\mathrm{Cu}$ \\
\hline S155 & $\mathrm{V}$ & $\mathrm{e}$ & Russo & 2017 & MFF & auto & $\mathrm{C}$ & 30 & None & No \\
\hline $\mathrm{S} 170$ & $\mathrm{~V}$ & $\mathrm{e}$ & Smyshlyaev & 2017 & ADRCs & auto & $\mathrm{C}$ & 28 & None & No \\
\hline S55 & V & $\mathrm{e}$ & Yokota & 2017 & ADRCs & auto & $\mathrm{C}$ & 13 & None & No \\
\hline S69 & $\mathrm{V}$ & $\mathrm{e}$ & Cattaneo & 2018 & $\mathrm{MFF}$ & auto & $\mathrm{C}$ & 38 & None & No \\
\hline S70 & $\mathrm{V}$ & $\mathrm{e}$ & Cavallo & 2018 & BMAC & auto & $\mathrm{C}+\mathrm{AD}+\mathrm{PRP}$ & 24 & None & No \\
\hline S153 & V & $\mathrm{e}$ & Rodriguez-Fontan & 2018 & BMAC & auto & $\mathrm{C}$ & 10 & None & No \\
\hline S156 & $\mathrm{V}$ & $\mathrm{e}$ & Russo & 2018 & $\mathrm{MFF}$ & auto & $\mathrm{C}$ & 30 & None & No \\
\hline S42 & $\mathrm{V}$ & e & Shaw & 2018 & BMAC & auto & $\mathrm{C}$ & 15 & None & No \\
\hline $\mathrm{S} 45$ & V & $\mathrm{e}$ & Spasovski & 2018 & ADSCs & auto & $\mathrm{C}$ & 9 & None & Yes \\
\hline S46 & $\mathrm{V}$ & $\mathrm{e}$ & Themistocleous & 2018 & BMAC & auto & $\mathrm{C}$ & 233 & None & No \\
\hline S62 & $\mathrm{V}$ & $\mathrm{e}$ & Borić & 2019 & MFF & auto & $\mathrm{C}$ & 17 & None & No \\
\hline S101 & V & $\mathrm{e}$ & Goncars & 2019 & BMAC & auto & $\mathrm{C}$ & 32 & None & No \\
\hline S17 & V & e & Hudetz & 2019 & MFF & auto & $\mathrm{C}$ & 20 & None & No \\
\hline S141 & $\mathrm{V}$ & e & Monckeberg & 2019 & pBSCs & auto & $\mathrm{C}+\mathrm{PRP}$ & 20 & None & Yes \\
\hline S143 & V & e & Onoi & 2019 & ADRCs & auto & $\mathrm{C}$ & 2 & None & No \\
\hline S37 & $\mathrm{V}$ & $\mathrm{e}$ & Roato & 2019 & CLL & auto & $\mathrm{C}+\mathrm{AD}$ & 20 & None & No \\
\hline S39 & V & $\mathrm{e}$ & Schiavone Panni & 2019 & MFF & auto & $\mathrm{C}+\mathrm{AD}$ & 52 & None & No \\
\hline S171 & $\mathrm{V}$ & $\mathrm{e}$ & Song & 2019 & hUCB-MSCs & allo & $\mathrm{C}+\mathrm{DFO}$ & 2 & None & Yes \\
\hline S183 & $\mathrm{V}$ & $\mathrm{e}$ & Wang & 2019 & BM-MSCs & auto & $\mathrm{C}+$ chondrocytes & 2 & None & Yes \\
\hline S79 & $\mathrm{V}$ & e & Colberg & 2020 & BMAC & auto & $\mathrm{C}$ & 10 & None & No \\
\hline S84 & $\mathrm{V}$ & $\mathrm{e}$ & Dilogo & 2020 & hUC-MSCs & allo & $\mathrm{C}$ & 39 & None & Yes \\
\hline S85 & $\mathrm{V}$ & $\mathrm{e}$ & Dulic & 2020 & BMAC & auto & $\mathrm{C}$ & 111 & None & No \\
\hline S95 & $\mathrm{V}$ & $\mathrm{e}$ & Freitag & 2020 & ADSCs & auto & $\mathrm{C}+\mathrm{AD}$ & 27 & None & Yes \\
\hline S104 & V & $\mathrm{e}$ & Heidari & 2020 & MFF & auto & $\mathrm{C}$ & 110 & None & No \\
\hline S108 & $\mathrm{V}$ & $\mathrm{e}$ & Higuchi & 2020 & ADSCs & auto & $\mathrm{C}$ & 34 & None & Yes \\
\hline S113 & $\mathrm{V}$ & $\mathrm{e}$ & Kim & 2020 & BMAC & auto & $\mathrm{C}$ & 25 & None & No \\
\hline S120 & $\mathrm{V}$ & $\mathrm{e}$ & Kim & 2020 & ADRCs & auto & $\mathrm{C}$ in $\mathrm{S}$ & 467 & None & No \\
\hline S127 & $\mathrm{V}$ & $\mathrm{e}$ & Lapuente & 2020 & ADRCs & auto & $\mathrm{C}$ & 50 & None & No \\
\hline S139 & $\mathrm{V}$ & $\mathrm{e}$ & Mehling & 2020 & ADRCs & auto & $\mathrm{C}+\mathrm{PRP}$ & 241 & None & No \\
\hline S151 & V & e & Prodromos & 2020 & MFF & auto & $\mathrm{C}+\mathrm{PRP}$ & 42 & None & No \\
\hline S172 & $\mathrm{V}$ & $\mathrm{e}$ & Song & 2020 & hUCB-MSCs & allo & $\mathrm{C}+\mathrm{AD}+\mathrm{MF}+\mathrm{HA}$ & 128 & None & Yes \\
\hline S173 & $\mathrm{V}$ & $\mathrm{e}$ & Song & 2020 & hUCB-MSCs & allo & $\mathrm{C}+\mathrm{MF}+\mathrm{HTO}+\mathrm{HA}$ & 25 & None & Yes \\
\hline S174 & $\mathrm{V}$ & $\mathrm{e}$ & Song & 2020 & hUCB-MSCs & allo & $\mathrm{C}+\mathrm{MF}+\mathrm{HTO}+\mathrm{HA}$ & 25 & None & Yes \\
\hline S176 & $\mathrm{V}$ & $\mathrm{e}$ & Toan & 2020 & BM-MSCs & auto & $\mathrm{C}+\mathrm{AD}$ & 46 & None & $?$ \\
\hline S48 & $\mathrm{V}$ & $\mathrm{e}$ & Tsubosaka & 2020 & ADRCs & auto & $\mathrm{C}$ & 57 & None & No \\
\hline S180 & V & e & Varady & 2020 & BMAC & auto & $\mathrm{C}$ & 17 & None & No \\
\hline S184 & $\mathrm{V}$ & $\mathrm{e}$ & Wells & 2020 & BMAC & auto & $\mathrm{C}$ & 11 & None & No \\
\hline S59 & $\mathrm{V}$ & $\mathrm{e}$ & Bakowski & 2021 & MFF & auto & $\mathrm{C}$ & 59 & None & No \\
\hline
\end{tabular}


Table S19 (cont.)

\begin{tabular}{|c|c|c|c|c|c|c|c|c|c|c|}
\hline $\mathbf{R}$ & $\mathbf{C a}$ & $\mathbf{T}$ & FA & $\mathbf{Y}$ & $\mathrm{Ce}$ & $\mathbf{O}$ & Treatment & $\mathbf{N}$ & Control & $\mathbf{C u}$ \\
\hline S61 & $\mathrm{V}$ & $\mathrm{e}$ & Borg & 2021 & MFF & auto & $\mathrm{C}$ & 456 & None & No \\
\hline S67 & $\mathrm{V}$ & $\mathrm{e}$ & Burnham & 2021 & BMAC & auto & $\mathrm{C}$ & 112 & None & No \\
\hline S68 & $\mathrm{V}$ & $\mathrm{e}$ & Caforio & 2021 & MFF & auto & $\mathrm{C}+\mathrm{AD}$ & 30 & None & No \\
\hline S103 & $\mathrm{V}$ & $\mathrm{e}$ & Heidari & 2021 & MFF & auto & $\mathrm{C}$ & 344 & None & No \\
\hline S122 & $\mathrm{V}$ & $\mathrm{e}$ & $\mathrm{Kim}$ & 2021 & ADRCs & auto & $\mathrm{C}+\mathrm{AD}+\mathrm{HTO}$ & 75 & None & No \\
\hline S137 & $\mathrm{V}$ & $\mathrm{e}$ & Malanga & 2021 & $\mathrm{MFF}$ & auto & $\mathrm{C}$ & 20 & None & No \\
\hline S160 & $\mathrm{V}$ & $\mathrm{e}$ & Santoprete & 2021 & ADRCs & auto & $\mathrm{C}$ & 84 & None & No \\
\hline S163 & $\mathrm{V}$ & $\mathrm{e}$ & Sekiya & 2021 & S-MSCs & auto & $\mathrm{C}$ & 8 & None & Yes \\
\hline S179 & $\mathrm{V}$ & e & Van Genechten & 2021 & MFF & auto & $\mathrm{C}$ & 64 & None & No \\
\hline S74 & $\mathrm{V}$ & $\mathrm{f}$ & Centeno & 2008 & BM-MSCs & auto & $\mathrm{C}$ & 1 & None & Yes \\
\hline S75 & $\mathrm{V}$ & $\mathrm{f}$ & Centeno & 2008 & BM-MSCs & auto & $\mathrm{C}$ & 1 & None & Yes \\
\hline $\mathrm{S} 140$ & $\mathrm{~V}$ & $\mathrm{f}$ & Mehrabani & 2016 & BM-MSCs & auto & $\mathrm{C}$ & 1 & None & Yes \\
\hline S63 & $\mathrm{V}$ & $\mathrm{f}$ & Bright & 2018 & ADRCs & auto & $\mathrm{C}$ & 1 & None & No \\
\hline S92 & $\mathrm{V}$ & $\mathrm{f}$ & Freitag & 2019 & ADSCs & auto & $\mathrm{C}+\mathrm{AD}+\mathrm{HTO}$ & 1 & None & Yes \\
\hline S182 & VI & $\mathrm{a}$ & Wakitani & 2002 & BM-MSCs & auto & $\mathrm{C}+\mathrm{AD}+\mathrm{HTO}+\mathrm{aP}$ & 12 & HTO & Yes \\
\hline S38 & VI & a & Saw & 2013 & pBSCs & auto & $\mathrm{C}+\mathrm{MF}+\mathrm{HA}$ & 25 & HA & No \\
\hline $\mathrm{S} 1$ & VI & a & Akgun & 2015 & S-MSCs & auto & $\mathrm{C}$ in $\mathrm{S}+\mathrm{AD}$ & 7 & MACI & Yes \\
\hline $\mathrm{S} 125$ & IV & $\mathrm{a}$ & Koh & 2016 & ADRCs & auto & $\mathrm{C}$ in $\mathrm{S}+\mathrm{MF}$ & 20 & MF & No \\
\hline S165 & VI & $\mathrm{a}$ & Shadmanfar & 2018 & BM-MSCs & auto & $\mathrm{C}$ & 15 & Placebo & Yes \\
\hline S83 & VI & $\mathrm{a}$ & de Girolamo & 2019 & BMAC & auto & $\mathrm{C}+\mathrm{AMIC}$ & 12 & AMIC & No \\
\hline $\mathrm{S} 15$ & VI & $\mathrm{a}$ & Hashimoto & 2019 & BM-MSCs & auto & $\mathrm{C}+\mathrm{MF}$ & 7 & MF & Yes \\
\hline $\mathrm{S} 142$ & VI & $\mathrm{a}$ & Olivos-Meza & 2019 & pBSCs & auto & $\mathrm{C}$ in $\mathrm{S}$ & 17 & $\mathrm{Sa}$ & Yes \\
\hline S117 & VI & $\mathrm{a}$ & Kim & 2020 & ADRCs & auto & $\mathrm{C}+\mathrm{AD}+\mathrm{HTO}$ & 40 & $\mathrm{C}+\mathrm{AD}+\mathrm{HTO}+\mathrm{Aca}$ & No \\
\hline $\mathrm{S} 133$ & VI & $\mathrm{a}$ & Lim & 2021 & hUCB-MSCs & allo & $\mathrm{C}+\mathrm{AD}+\mathrm{MF}$ & 73 & MF & Yes \\
\hline $\mathrm{S} 162$ & VI & a & Saw & 2021 & pBSCs & auto & $\mathrm{C}+\mathrm{MF}+\mathrm{HA}$ & 35 & $\mathrm{HA}$ & No \\
\hline S97 & VI & $\mathrm{c}$ & Gobbi & 2015 & BMAC & auto & $\mathrm{C}$ in $\mathrm{S}+\mathrm{AD}$ & 19 & MACI & $\mathrm{No}$ \\
\hline $\mathrm{S} 100$ & VI & $\mathrm{c}$ & Gobbi & 2016 & BMAC & auto & $\mathrm{C}$ in $\mathrm{S}$ & 27 & $\mathrm{MF}$ & No \\
\hline S138 & VI & c & Martinčič & 2020 & BMAC & auto & $\mathrm{C}$ in $\mathrm{S}$ & 9 & autoCh & No \\
\hline $\mathrm{S} 157$ & VI & d & Ryu & 2020 & $\mathrm{BMAC}$ & auto & $\mathrm{C}+\mathrm{AD}$ & 52 & hUC-MSCs & No \\
\hline $\mathrm{S} 111$ & VI & $\mathrm{e}$ & Kasemkijwattana & 2011 & BM-MSCs & auto & $\mathrm{C}$ in $\mathrm{S}$ & 2 & None & Yes \\
\hline S169 & VI & $\mathrm{e}$ & Skowroński & 2012 & pBSCs & auto & $\mathrm{C}$ in $\mathrm{S}+\mathrm{AD}$ & 52 & None & No \\
\hline S65 & VI & $\mathrm{e}$ & Buda & 2013 & BMAC & auto & $\mathrm{C}$ in $\mathrm{S}+\mathrm{AD}$ & 20 & None & No \\
\hline S98 & VI & $\mathrm{e}$ & Gobbi & 2014 & BMAC & auto & $\mathrm{C}$ in $\mathrm{S}+\mathrm{AD}$ & 25 & None & No \\
\hline S186 & VI & $\mathrm{e}$ & Zhang & 2014 & MACI & auto & $\mathrm{C}$ in $\mathrm{S}+\mathrm{AD}$ & 15 & None & Yes \\
\hline S109 & VI & $\mathrm{e}$ & Jiang & 2016 & $\mathrm{CSCs}$ & auto & $\mathrm{C}$ in $\mathrm{S}+\mathrm{AD}$ & 15 & None & Yes \\
\hline $\mathrm{S} 158$ & VI & $\mathrm{e}$ & Sadlik & 2017 & hUC-MSCs & allo & $\mathrm{C}$ in $\mathrm{S}$ & $?$ & None & Yes \\
\hline S185 & VI & $\mathrm{e}$ & Whitehouse & 2017 & BM-MSCs & auto & $\mathrm{C}$ in $\mathrm{S}$ & 5 & None & Yes \\
\hline $\mathrm{S} 110$ & VI & $\mathrm{e}$ & Kamei & 2018 & BM-MSCs & auto & $\mathrm{C}$ & 5 & None & Yes \\
\hline
\end{tabular}




\section{Table S19 (cont.)}

\begin{tabular}{|c|c|c|c|c|c|c|c|c|c|c|}
\hline $\mathbf{R}$ & $\mathbf{C a}$ & $\mathbf{T}$ & $\mathbf{F A}$ & $\mathbf{Y}$ & $\mathrm{Ce}$ & $\mathbf{O}$ & Treatment & $\mathbf{N}$ & Control & $\mathbf{C u}$ \\
\hline S166 & VI & $\mathrm{e}$ & Shetty & 2018 & BMAC & auto & $\mathrm{C}+\mathrm{AD}+\mathrm{MF}$ & 60 & None & No \\
\hline S167 & VI & $\mathrm{e}$ & Shimomura & 2018 & S-MSCs & auto & $\mathrm{C}$ & 5 & None & Yes \\
\hline S99 & VI & $\mathrm{e}$ & Gobbi & 2019 & BMAC & auto & $\mathrm{C}$ in $\mathrm{S}+\mathrm{AD}$ & 23 & None & No \\
\hline S164 & VI & $\mathrm{e}$ & Sekiya & 2019 & S-MSCs & auto & $\mathrm{C}+\mathrm{SR}$ & 6 & None & Yes \\
\hline S78 & VI & $\mathrm{e}$ & Ciemniewska-Gorzela & 2020 & BMAC & auto & $\mathrm{C}$ in $\mathrm{S}+\mathrm{AM}$ & 54 & None & No \\
\hline S94 & VI & $\mathrm{e}$ & Freitag & 2020 & ADSCs & auto & $\mathrm{C}+\mathrm{AD}$ & 8 & None & Yes \\
\hline S181 & VI & $\mathrm{e}$ & Veber & 2020 & BMAC & auto & $\mathrm{C}$ in $\mathrm{S}$ & 15 & None & No \\
\hline S77 & VI & $\mathrm{e}$ & Chung & 2021 & hUCB-MSCs & allo & $\mathrm{C}+\mathrm{AD}+\mathrm{HTO}$ & 93 & None & Yes \\
\hline S134 & VI & $\mathrm{e}$ & Liu & 2021 & $\mathrm{Cs} / \mathrm{CPs}$ & auto & $\mathrm{C}$ in $\mathrm{S}+\mathrm{AD}$ & 12 & None & Yes \\
\hline S161 & VI & e & Saris & 2021 & BM-MSCs & allo & $C+A D$ & 35 & None & Yes \\
\hline S57 & VI & $\mathrm{f}$ & Adachi & 2005 & BM-MSCs & auto & $\mathrm{C}$ in $\mathrm{S}$ & 1 & None & Yes \\
\hline S126 & VI & $\mathrm{f}$ & Kuroda & 2007 & BM-MSCs & auto & $\mathrm{C}$ in $\mathrm{S}$ & 1 & None & Yes \\
\hline S64 & VI & $\mathrm{f}$ & Broyles & 2017 & BMAC & auto & $\mathrm{C}+\mathrm{PRP}+\mathrm{HA}$ & 1 & None & No \\
\hline S93 & VI & $\mathrm{f}$ & Freitag & 2017 & ADSCs & auto & $\mathrm{C}$ & 1 & None & Yes \\
\hline S91 & VI & $\mathrm{f}$ & Freitag & 2017 & ADSCs & auto & $\mathrm{C}$ & 1 & None & Yes \\
\hline $\mathrm{S} 131$ & VI & $\mathrm{f}$ & Leigheb & 2017 & $\mathrm{Cs}$ & auto & $\mathrm{C}$ in $\mathrm{S}$ & 1 & None & Yes \\
\hline
\end{tabular}


Table S20 | Categories of studies on treatment of primary knee osteoarthritis (pkOA) with stem cells that were identified during an evidence-based, systematic review of the literature according to the PRISMA guidelines ${ }^{14}$ performed on August 07, 2021 (summarized in Table S19).

Abbreviations: $\mathrm{Ca}=$ category; $\mathrm{CS}=$ corticosteroid; $\mathrm{N}=$ number of studies; $\mathrm{HA}=$ hyaluronic acid; i.a. $=$ intra-articular; PRP = platelet rich plasma.

\begin{tabular}{ccl}
\hline Ca & N & Description \\
\hline I & 8 & $\begin{array}{l}\text { Treatment of pkOA with i.a. injection of stem cells as the sole treatment (not } \\
\text { considering rehabilitation), compared with i.a. injection of saline or sham treatment } \\
\text { as control. }\end{array}$ \\
II & 8 & $\begin{array}{l}\text { Treatment of pkOA with i.a. injection of stem cells as the sole treatment (not } \\
\text { considering rehabilitation), compared with i.a. injection of respectively PRP, CS or }\end{array}$ \\
III & 22 & $\begin{array}{l}\text { HA as control. } \\
\text { Treatment of pkOA with i.a. injection of stem cells as the sole treatment (not } \\
\text { considering rehabilitation), compared with other treatments than those in Categories I } \\
\text { and II as control. } \\
\text { Treatment of pkOA with combinations of stem cells and other modalities, compared } \\
\text { with sham treatment or other treatments as control. } \\
\text { Treatment of pkOA with combinations of stem cells with or without other modalities, } \\
\text { without control group (case series or case reports). } \\
\text { Treatment of focal chondral, osteochondral or meniscal chondral lesions with stem } \\
\text { cells as the sole treatment (not considering rehabilitation) or combinations of stem } \\
\text { cells and other modalities, with or without other treatments as control. }\end{array}$ \\
V & 27 &
\end{tabular}


Table S21 | Types of studies on treatment of primary knee osteoarthritis (pkOA) with stem cells that were identified during an evidence-based, systematic review of the literature according to the PRISMA guidelines ${ }^{14}$ performed on August 07, 2021.

Abbreviations: $\mathrm{Ca}=$ category; $\mathrm{N}_{\text {all }}=$ number of studies among all studies listed in Table $\mathrm{S} 19 ; \mathrm{N}_{\mathrm{pkOA}}=$ number of studies among those studies listed in Table S19 that addressed pkOA.

\begin{tabular}{cccl}
\hline Ca & $\mathbf{N}_{\text {all }}$ & $\mathbf{N}_{\mathbf{p k O A}}$ & Description \\
\hline a & 44 & 33 & Randomized controlled trials (RCTs) \\
b & 6 & 6 & RCTs with the contralateral knee as internal control \\
c & 18 & 15 & Prospective cohort studies \\
d & 12 & 11 & Retrospective cohort studies \\
e & 92 & 73 & Case series with more than one subject \\
f & 11 & 5 & Case reports with only one subject \\
\hline Sum & 183 & 143 & \\
\hline
\end{tabular}


Table S22 | Types of stem cells used in studies on treatment of primary knee osteoarthritis pkOA with stem cells that were identified during an evidence-based, systematic review of the literature according to the PRISMA guidelines ${ }^{14}$ performed on August 07, 2021.

Abbreviations: $\mathrm{N}_{\mathrm{all}}=$ number of studies among all studies listed in Table S19; $\mathrm{N}_{\mathrm{pkOA}}=$ number of studies among those studies listed in Table S19 that addressed pkOA.

\begin{tabular}{lccl}
\hline \multicolumn{1}{c}{ Cell type } & $\mathbf{N}_{\text {all }}$ & $\mathbf{N}_{\mathbf{p k O A}}$ & Description \\
\hline ADRCs & 36 & 35 & Adipose-derived regenerative cells \\
ADSCs & 22 & 18 & Adipose-derived stem cells (obtained by culturing ADRCs) \\
MFF & 21 & 21 & Micro-fragmented fat (from liposuction) \\
CLL & 1 & 1 & Centrifuged liposuction liquid \\
\hline BMA & 1 & 1 & Bone marrow aspirate \\
BMAC & 38 & 26 & Bone marrow aspirate concentrate \\
BM-MSCs & 34 & 25 & Bone marrow-derived mesenchymal stem cells \\
\hline Cs/CPs & 2 & 0 & Chondrocytes and chondrocyte precursors \\
CSCs & 1 & 0 & Cartilage stem cells \\
MACI & 1 & 0 & Matrix-induced, autologous chondrocyte implant \\
S-MSCs & 4 & 1 & Matrix-induced MSCs from synovia \\
Ch-TGFB & 2 & 2 & Chondrocytes that overexpress transcription growth factor beta \\
\hline hUC-MSCs & 4 & 3 & Human umbilical cord-derived MSCs \\
hUCB-MSCs & 7 & 5 & Human umbilical cord blood-derived MSCs \\
P-MSCs & 1 & 1 & Placental MSCs \\
pBSCs & 8 & 4 & Activated peripheral blood stem cells \\
\hline Sum & 183 & 143 & \\
\hline
\end{tabular}




\section{References}

S1. Akgun I, Unlu MC, Erdal OA, Ogut T, Erturk M, Ovali E, Kantarci F, Caliskan G, Akgun Y. Matrix-induced autologous mesenchymal stem cell implantation versus matrix-induced autologous chondrocyte implantation in the treatment of chondral defects of the knee: a 2-year randomized study. Arch Orthop Trauma Surg. 2015;135(2):251-263.

S2. Al-Najar M, Khalil H, Al-Ajlouni J, Al-Antary E, Hamdan M, Rahmeh R, Alhattab D, Samara O, Yasin M, Abdullah AA, Al-Jabbari E, Hmaid D, Jafar H, Awidi A. Intra-articular injection of expanded autologous bone marrow mesenchymal cells in moderate and severe knee osteoarthritis is safe: a phase I/II study. J Orthop Surg Res. 2017;12(1):190.

S3. Anz AW, Hubbard R, Rendos NK, Everts PA, Andrews JR, Hackel JG. Bone marrow aspirate concentrate is equivalent to platelet-rich plasma for the treatment of knee osteoarthritis at 1 year: a prospective, randomized trial. Orthop J Sports Med. 2020;8(2):2325967119900958.

S4. Bastos R, Mathias M, Andrade R, Amaral RJFC, Schott V, Balduino A, Bastos R, Miguel Oliveira $\mathrm{J}$, Reis RL, Rodeo S, Espregueira-Mendes J. Intra-articular injection of culture-expanded mesenchymal stem cells with or without addition of platelet-rich plasma is effective in decreasing pain and symptoms in knee osteoarthritis: a controlled, double-blind clinical trial. Knee Surg Sports Traumatol Arthrosc. 2020;28(6):1989-1999.

S5. Bastos R, Mathias M, Andrade R, Bastos R, Balduino A, Schott V, Rodeo S, Espregueira-Mendes J. Intra-articular injections of expanded mesenchymal stem cells with and without addition of platelet-rich plasma are safe and effective for knee osteoarthritis. Knee Surg Sports Traumatol Arthrosc. 2018;26(11):3342-3350.

S6. Bhattacharya N. Clinical use of amniotic fluid in osteoarthritis: a source of cell therapy. Transplantation 2011;90:395-403.

S7. Cherian JJ, Parvizi J, Bramlet D, Lee KH, Romness DW, Mont MA. Preliminary results of a phase II randomized study to determine the efficacy and safety of genetically engineered allogeneic human chondrocytes expressing TGF- $\beta 1$ in patients with grade 3 chronic degenerative joint disease of the knee. Osteoarthritis Cartilage. 2015;23(12):2109-2118.

S8. Emadedin M, Labibzadeh N, Liastani MG, Karimi A, Jaroughi N, Bolurieh T, Hosseini SE, Baharvand $\mathrm{H}$, Aghdami N. Intra-articular implantation of autologous bone marrow-derived mesenchymal stromal cells to treat knee osteoarthritis: a randomized, triple-blind, placebocontrolled phase 1/2 clinical trial. Cytotherapy. 2018;20(10):1238-1246.

S9. Freitag J, Bates D, Wickham J, Shah K, Huguenin L, Tenen A, Paterson K, Boyd R. Adiposederived mesenchymal stem cell therapy in the treatment of knee osteoarthritis: a randomized controlled trial. Regen Med. 2019;14(3):213-230.

S10. Garay-Mendoza D, Villarreal-Martínez L, Garza-Bedolla A, Pérez-Garza DM, Acosta-Olivo C, Vilchez-Cavazos F, Diaz-Hutchinson C, Gómez-Almaguer D, Jaime-Pérez JC, Mancías-Guerra C. The effect of intra-articular injection of autologous bone marrow stem cells on pain and knee function in patients with osteoarthritis. Int J Rheum Dis. 2018;21(1):140-147.

S11. Garza JR, Campbell RE, Tjoumakaris FP, Freedman KB, Miller LS, Santa Maria D, Tucker BS. Clinical efficacy of intra-articular mesenchymal stromal cells for the treatment of knee osteoarthritis: a double-blinded prospective randomized controlled clinical trial. Am J Sports Med. 2020;48(3):588-598.

S12. Goncars V, Jakobsons E, Blums K, Briede I, Patetko L, Erglis K, Erglis M, Kalnberzs K, Muiznieks I, Erglis A. The comparison of knee osteoarthritis treatment with single-dose bone marrow-derived mononuclear cells vs. hyaluronic acid injections. Medicina (Kaunas). 2017;53(2):101-108.

S13. Gupta PK, Chullikana A, Rengasamy M, Shetty N, Pandey V, Agarwal V, Wagh SY, Vellotare PK, Damodaran D, Viswanathan P, Thej C, Balasubramanian S, Majumdar AS. Efficacy and safety of adult human bone marrow-derived, cultured, pooled, allogeneic mesenchymal stromal cells (Stempeucel®): preclinical and clinical trial in osteoarthritis of the knee joint. Arthritis Res Ther. 2016;18(1):301. 
S14. Ha CZ, Li W, Ren SD, Zhou CH, Chen SF, Wang DW, et al. Effect of platelet rich plasma combined with mesenchymal stem cells in treatment of knee osteoarthritis. Chin J Joint Surg (Electronic Edition). 2018;12(5):644-652.

S15. Hashimoto Y, Nishida Y, Takahashi S, Nakamura H, Mera H, Kashiwa K, Yoshiya S, Inagaki Y, Uematsu K, Tanaka Y, Asada S, Akagi M, Fukuda K, Hosokawa Y, Myoui A, Kamei N, Ishikawa M, Adachi N, Ochi M, Wakitani S. Transplantation of autologous bone marrow-derived mesenchymal stem cells under arthroscopic surgery with microfracture versus microfracture alone for articular cartilage lesions in the knee: a multicenter prospective randomized control clinical trial. Regen Ther. 2019;11:106-113.

S16. Hong Z, Chen J, Zhang S, Zhao C, Bi M, Chen X, Bi Q. Intra-articular injection of autologous adipose-derived stromal vascular fractions for knee osteoarthritis: a double-blind randomized selfcontrolled trial. Int Orthop. 2019;43(5):1123-1134.

S17. Hudetz D, Borić I, Rod E, Jeleč Ž, Kunovac B, Polašek O, Vrdoljak T, Plečko M, Skelin A, Polančec D, Zenić L, Primorac D. Early results of intra-articular micro-fragmented lipoaspirate treatment in patients with late stages knee osteoarthritis: a prospective study. Croat Med J. 2019;60(3):227-236.

S18. Hudetz D, Borić I, Rod E, Jeleč Ž, Radić A, Vrdoljak T, Skelin A, Lauc G, Trbojević-Akmačić I, Plečko M, Polašek O, Primorac D. The effect of intra-articular injection of autologous microfragmented fat tissue on proteoglycan synthesis in patients with knee osteoarthritis. Genes. 2017;8(10):270.

S19. Jo CH, Chai JW, Jeong EC, Oh S, Shin JS, Shim H, Yoon KS. Intra-articular injection of mesenchymal stem cells for the treatment of osteoarthritis of the knee: a 2-year follow-up study. Am J Sports Med. 2017;45(12):2774-2783.

S20. Jo CH, Lee YG, Shin WH, Kim H, Chai JW, Jeong EC, Kim JE, Shim H, Shin JS, Shin IS, Ra JC, $\mathrm{Oh}$ S, Yoon KS. Intra-articular injection of mesenchymal stem cells for the treatment of osteoarthritis of the knee: a proof-of-concept clinical trial. Stem Cells. 2014;32(5):1254-66.

S21. Khalifeh Soltani S, Forogh B, Ahmadbeigi N, Hadizadeh Kharazi H, Fallahzadeh K, Kashani L, Karami M, Kheyrollah Y, Vasei M. Safety and efficacy of allogenic placental mesenchymal stem cells for treating knee osteoarthritis: a pilot study. Cytotherapy. 2019;21(1):54-63.

S22. Kim MK, Ha CW, In Y, Cho SD, Choi ES, Ha JK, Lee JH, Yoo JD, Bin SI, Choi CH, Kyung HS, Lee MC. A multicenter, double-blind, phase iii clinical trial to evaluate the efficacy and safety of a cell and gene therapy in knee osteoarthritis patients. Hum Gene Ther Clin Dev. 2018;29(1):48-59.

S23. Koh YG, Choi YJ. Infrapatellar fat pad-derived mesenchymal stem cell therapy for knee osteoarthritis. Knee. 2012;19(6):902-7.

S24. Koh YG, Jo SB, Kwon OR, Suh DS, Lee SW, Park SH, Choi YJ. Mesenchymal stem cell injections improve symptoms of knee osteoarthritis. Arthroscopy. 2013;29(4):748-55.

S25. Koh YG, Kwon OR, Kim YS, Choi YJ. Comparative outcomes of open-wedge high tibial osteotomy with platelet-rich plasma alone or in combination with mesenchymal stem cell treatment: a prospective study. Arthroscopy. 2014;30(11):1453-60.

S26. Kuah D, Sivell S, Longworth T, James K, Guermazi A, Cicuttini F, Wang Y, Craig S, Comin G, Robinson D, Wilson J. Safety, tolerability and efficacy of intra-articular Progenza in knee osteoarthritis: a randomized double-blind placebo-controlled single ascending dose study. J Transl Med. 2018;16(1):49.

S27. Lamo-Espinosa JM, Mora G, Blanco JF, Granero-Moltó F, Nuñez-Córdoba JM, SánchezEchenique C, Bondía JM, Aquerreta JD, Andreu EJ, Ornilla E, Villarón EM, Valentí-Azcárate A, Sánchez-Guijo F, Del Cañizo MC, Valentí-Nin JR, Prósper F. Intra-articular injection of two different doses of autologous bone marrow mesenchymal stem cells versus hyaluronic acid in the treatment of knee osteoarthritis: multicenter randomized controlled clinical trial (phase I/II). J Transl Med. 2016;14(1):246.

S28. Lee WS, Kim HJ, Kim KI, Kim GB, Jin W. Intra-articular injection of autologous adipose tissuederived mesenchymal stem cells for the treatment of knee osteoarthritis: a Phase IIb, randomized, 
placebo-controlled clinical trial. Stem Cells Transl Med. 2019;8(6):504-511.

S29. Lu L, Dai C, Zhang Z, Du H, Li S, Ye P, Fu Q, Zhang L, Wu X, Dong Y, Song Y, Zhao D, Pang Y, Bao C. Treatment of knee osteoarthritis with intra-articular injection of autologous adiposederived mesenchymal progenitor cells: a prospective, randomized, double-blind, active-controlled, phase IIb clinical trial. Stem Cell Res Ther. 2019;10(1):143.

S30. Lv XX, Huang C, Yin Z, Hong BG, Jiang HJ, Huang XJ. Effectiveness of autologous bone marrow mesenchymal stem cell transplant for knee osteoarthritis. Chin J Cell Stem Cell. 2015;5(2):28-32.

S31. Matas J, Orrego M, Amenabar D, Infante C, Tapia-Limonchi R, Cadiz MI, Alcayaga-Miranda F, González PL, Muse E, Khoury M, Figueroa FE, Espinoza F. Umbilical cord-derived mesenchymal stromal cells (MSCs) for knee osteoarthritis: repeated MSC dosing is superior to a single msc dose and to hyaluronic acid in a controlled randomized Phase I/II trial. Stem Cells Transl Med. 2019;8(3):215-224.

S32. Mautner K, Bowers R, Easley K, Fausel Z, Robinson R. Functional outcomes following microfragmented adipose tissue versus bone marrow aspirate concentrate injections for symptomatic knee osteoarthritis. Stem Cells Transl Med. 2019;8(11):1149-1156.

S33. Nguyen PD, Tran TD, Nguyen HT, Vu HT, Le PT, Phan NL, Vu NB, Phan NK, Van Pham P. Comparative clinical observation of arthroscopic microfracture in the presence and absence of a stromal vascular fraction injection for osteoarthritis. Stem Cells Transl Med. 2017;6(1):187-195.

S34. Orozco L, Munar A, Soler R, Alberca M, Soler F, Huguet M, Sentís J, Sánchez A, García-Sancho J. Treatment of knee osteoarthritis with autologous mesenchymal stem cells: a pilot study. Transplantation. 2013;95(12):1535-41.

S35. Pers YM, Quentin J, Feirreira R, Espinoza F, Abdellaoui N, Erkilic N, Cren M, Dufourcq-Lopez E, Pullig O, Nöth U, Jorgensen C, Louis-Plence P. Injection of adipose-derived stromal cells in the knee of patients with severe osteoarthritis has a systemic effect and promotes an anti-inflammatory phenotype of circulating immune cells. Theranostics. 2018;8(20):5519-5528.

S36. Pers YM, Rackwitz L, Ferreira R, Pullig O, Delfour C, Barry F, Sensebe L, Casteilla L, Fleury S, Bourin P, Noël D, Canovas F, Cyteval C, Lisignoli G, Schrauth J, Haddad D, Domergue S, Noeth U, Jorgensen C; ADIPOA Consortium. Adipose mesenchymal stromal cell-based therapy for severe osteoarthritis of the knee: a phase i dose-escalation trial. Stem Cells Transl Med. 2016;5(7):847-56.

S37. Roato I, Belisario DC, Compagno M, Lena A, Bistolfi A, Maccari L, Mussano F, Genova T, Godio L, Perale G, Formica M, Cambieri I, Castagnoli C, Robba T, Felli L, Ferracini R. Concentrated adipose tissue infusion for the treatment of knee osteoarthritis: clinical and histological observations. Int Orthop. 2019;43(1):15-23.

S38. Saw KY, Anz A, Siew-Yoke Jee C, Merican S, Ching-Soong Ng R, Roohi SA, Ragavanaidu K. Articular cartilage regeneration with autologous peripheral blood stem cells versus hyaluronic acid: a randomized controlled trial. Arthroscopy. 2013r;29(4):684-94.

S39. Schiavone Panni A, Vasso M, Braile A, Toro G, De Cicco A, Viggiano D, Lepore F. Preliminary results of autologous adipose-derived stem cells in early knee osteoarthritis: identification of a subpopulation with greater response. Int Orthop. 2019;43(1):7-13.

S40. Shapiro SA, Arthurs JR, Heckman MG, Bestic JM, Kazmerchak SE, Diehl NN, Zubair AC, O'Connor MI. Quantitative T2 MRI mapping and 12-month follow-up in a randomized, blinded, placebo controlled trial of bone marrow aspiration and concentration for osteoarthritis of the knees. Cartilage. 2019;10(4):432-443.

S41. Shapiro SA, Kazmerchak SE, Heckman MG, Zubair AC, O'Connor MI. A prospective, singleblind, placebo-controlled trial of bone marrow aspirate concentrate for knee osteoarthritis. Am J Sports Med. 2017;45(1):82-90.

S42. Shaw B, Darrow M, Derian A. Short-term outcomes in treatment of knee osteoarthritis with 4 bone marrow concentrate injections. Clin Med Insights Arthritis Musculoskelet Disord. 2018;11:1179544118781080.

S43. Soler R, Orozco L, Munar A, Huguet M, López R, Vives J, Coll R, Codinach M, Garcia-Lopez J. 
Final results of a phase I-II trial using ex vivo expanded autologous Mesenchymal Stromal Cells for the treatment of osteoarthritis of the knee confirming safety and suggesting cartilage regeneration. Knee. 2016;23(4):647-54.

S44. Song Y, Du H, Dai C, Zhang L, Li S, Hunter DJ, Lu L, Bao C. Human adipose-derived mesenchymal stem cells for osteoarthritis: a pilot study with long-term follow-up and repeated injections. Regen Med. 2018;13(3):295-307.

S45. Spasovski D, Spasovski V, Baščarević Z, Stojiljković M, Vreća M, Anđelković M, Pavlović S. Intra-articular injection of autologous adipose-derived mesenchymal stem cells in the treatment of knee osteoarthritis. J Gene Med. 2018 Jan;20(1).

S46. Themistocleous GS, Chloros GD, Kyrantzoulis IM, Georgokostas IA, Themistocleous MS, Papagelopoulos PJ, Savvidou OD. Effectiveness of a single intra-articular bone marrow aspirate concentrate (BMAC) injection in patients with grade 3 and 4 knee osteoarthritis. Heliyon. 2018;4(10):e00871.

S47. Tran TDX, Wu CM, Dubey NK, Deng YH, Su CW, Pham TT, Thi Le PB, Sestili P, Deng WP. Time- and Kellgren-Lawrence grade-dependent changes in intra-articularly transplanted stromal vascular fraction in osteoarthritic patients. Cells. 2019;8(4):308.

S48. Tsubosaka M, Matsumoto T, Sobajima S, Matsushita T, Iwaguro H, Kuroda R. The influence of adipose-derived stromal vascular fraction cells on the treatment of knee osteoarthritis. BMC Musculoskelet Disord. 2020;21(1):207.

S49. Vangsness CT Jr, Farr J 2nd, Boyd J, Dellaero DT, Mills CR, LeRoux-Williams M. Adult human mesenchymal stem cells delivered via intra-articular injection to the knee following partial medial meniscectomy: a randomized, double-blind, controlled study. J Bone Joint Surg Am. 2014;96(2):90-98.

S50. Varma HS, Dadarya B, Vidyarthi A. The new avenues in the management of osteo-arthritis of knee-stem cells. J Indian Med Assoc. 2010;108(9):583-585.

S51. Vega A, Martín-Ferrero MA, Del Canto F, Alberca M, García V, Munar A, Orozco L, Soler R, Fuertes JJ, Huguet M, Sánchez A, García-Sancho J. Treatment of knee osteoarthritis with allogeneic bone marrow mesenchymal stem cells: a randomized controlled trial. Transplantation. 2015;99(8):1681-90.

S52. Wang Y, Jin W, Liu H, Cui Y, Mao Q, Fei Z, Xiang C. [Curative effect of human umbilical cord mesenchymal stem cells by intra-articular injection for degenerative knee osteoarthritis]. Zhongguo Xiu Fu Chong Jian Wai Ke Za Zhi. 2016;30(12):1472-1477. Chinese.

S53. Wong KL, Lee KB, Tai BC, Law P, Lee EH, Hui JH. Injectable cultured bone marrow-derived mesenchymal stem cells in varus knees with cartilage defects undergoing high tibial osteotomy: a prospective, randomized controlled clinical trial with 2 years' follow-up. Arthroscopy. 2013;29(12):2020-8.

S54. Yokota N, Hattori M, Ohtsuru T, Otsuji M, Lyman S, Shimomura K, Nakamura N. Comparative clinical outcomes after intra-articular injection with adipose-derived cultured stem cells or noncultured stromal vascular fraction for the treatment of knee osteoarthritis. Am J Sports Med. 2019;47(11):2577-2583.

S55. Yokota N, Yamakawa M, Shirata T, Kimura T, Kaneshima H. Clinical results following intraarticular injection of adipose-derived stromal vascular fraction cells in patients with osteoarthritis of the knee. Regen Ther. 2017;6:108-112.

S56. Zhang SY, Lyu SJ, Ding QW, Fan MQ, Tong PJ. Intraarticular injection of autologous adiposederived stem cells for knee osteoarthritis: A randomized controlled trial [in Chinese]. Zhong Hua Gu Ke Za Zhi. 2018;38:1426-1434.

S57. Adachi N, Ochi M, Deie M, Ito Y. Transplant of mesenchymal stem cells and hydroxyapatite ceramics to treat severe osteochondral damage after septic arthritis of the knee. J Rheumatol. 2005;32(8):1615-8.

S58. Ahmad KA, Ibrahim YA, Saber NZ, Darwish BA. MR cartilage imaging in assessment of the regenerative power of autologous peripheral blood stem cell injection in knee osteoarthritis. Eypt $\mathbf{J}$ 
Radiol Nucl Med 2014;45(3):787-794.

S59. Bąkowski P, Kaszyński J, Baka C, Kaczmarek T, Ciemniewska-Gorzela K, Bąkowska-Żywicka K, Piontek T. Patients with stage II of the knee osteoarthritis most likely benefit from the intra-articular injections of autologous adipose tissue-from 2 years of follow-up studies. Arch Orthop Trauma Surg. 2021 Jun 11. Epub ahead of print.

S60. Bistolfi A, Roato I, Fornelli G, Sabatini L, Massè A, Ferracini R. Treatment of knee osteoarthritis by intra-articular injection of concentrated autologous adipose tissue: a twenty four month followup study. Int Orthop. 2021;45(3):627-633.

S61. Borg TM, Heidari N, Noorani A, Slevin M, Cullen A, Olgiati S, Zerbi A, Danovi A, Wilson A. Gender-specific response in pain and function to biologic treatment of knee osteoarthritis: a genderbias-mitigated, observational, intention-to-treat study at two years. Stem Cells Int. 2021;2021:6648437.

S62. Borić I, Hudetz D, Rod E, Jeleč Ž, Vrdoljak T, Skelin A, Polašek O, Plečko M, Trbojević-Akmačić I, Lauc G, Primorac D. A 24-month follow-up study of the effect of intra-articular injection of autologous microfragmented fat tissue on proteoglycan synthesis in patients with knee osteoarthritis. Genes. 2019;10(12):1051.

S63. Bright B, Bright R, Bright P, Limaye A. Ankylosing spondylitis, chronic fatigue and depression improved after stromal vascular fraction treatment for osteoarthritis: a case report. J Med Case Rep. 2018;12(1):238.

S64. Broyles JE, O'Brien MA, Stagg MP. Microdrilling surgery augmented with intra-articular bone marrow aspirate concentrate, platelet-rich plasma, and hyaluronic acid: a technique for cartilage repair in the knee. Arthrosc Tech. 2017;6(1):S201-S206.

S65. Buda R, Vannini F, Cavallo M, Baldassarri M, Luciani D, Mazzotti A, Pungetti C, Olivieri A, Giannini S. One-step arthroscopic technique for the treatment of osteochondral lesions of the knee with bone-marrow-derived cells: three years results. Musculoskelet Surg. 2013;97(2):145-51.

S66. Bui KHT, Duong TD, Nguyen NT, Nguyen TC. Symptomatic knee osteoarthritis treatment using autologous adipose derived stem cells and platelet-rich plasma: a clinical study. Biomed Res Ther 2014;1(1):2-8.

S67. Burnham R, Smith A, Hart D. The safety and effectiveness of bone marrow concentrate injection for knee and hip osteoarthritis: a Canadian cohort. Regen Med. 2021;16(7):619-628.

S68. Caforio M, Nobile C. Intra-articular administration of autologous purified adipose tissue associated with arthroscopy ameliorates knee osteoarthritis symptoms. J Clin Med. 2021;10(10):2053.

S69. Cattaneo G, De Caro A, Napoli F, Chiapale D, Trada P, Camera A. Micro-fragmented adipose tissue injection associated with arthroscopic procedures in patients with symptomatic knee osteoarthritis. BMC Musculoskelet Disord. 2018;19(1):176.

S70. Cavallo M, Sayyed-Hosseinian SH, Parma A, Buda R, Mosca M, Giannini S. Combination of high tibial osteotomy and autologous bone marrow derived cell implantation in early osteoarthritis of knee: a preliminary study. Arch Bone Jt Surg. 2018;6(2):112-118.

S71. Centeno C, Cartier C, Stemper I, Dodson E, Freeman M, Azuike U, Williams C, Hyzy M, Silva O, Steinmetz N. The treatment of bone marrow lesions associated with advanced knee osteoarthritis: comparing intraosseous and intraarticular injections with bone marrow concentrate and platelet products. Pain Physician. 2021;24(3):S279-S288.

S72. Centeno C, Pitts J, Al-Sayegh H, Freeman M. Efficacy of autologous bone marrow concentrate for knee osteoarthritis with and without adipose graft. Biomed Res Int. 2014;2014:370621.

S73. Centeno CJ, Al-Sayegh H, Bashir J, Goodyear S, Freeman MD. A dose response analysis of a specific bone marrow concentrate treatment protocol for knee osteoarthritis. BMC Musculoskelet Disord. 2015;16:258.

S74. Centeno CJ, Busse D, Kisiday J, Keohan C, Freeman M, Karli D. Increased knee cartilage volume in degenerative joint disease using percutaneously implanted, autologous mesenchymal stem cells. Pain Physician. 2008;11(3):343-53.

S75. Centeno CJ, Busse D, Kisiday J, Keohan C, Freeman M. Increased knee cartilage volume in 
degenerative joint disease using percutaneously implanted, autologous mesenchymal stem cells, platelet lysate and dexamethasone. Am J Case Rep 2008;9:246-251.

S76. Chahal J, Gómez-Aristizábal A, Shestopaloff K, Bhatt S, Chaboureau A, Fazio A, Chisholm J, Weston A, Chiovitti J, Keating A, Kapoor M, Ogilvie-Harris DJ, Syed KA, Gandhi R, Mahomed NN, Marshall KW, Sussman MS, Naraghi AM, Viswanathan S. Bone marrow mesenchymal stromal cell treatment in patients with osteoarthritis results in overall improvement in pain and symptoms and reduces synovial inflammation. Stem Cells Transl Med. 2019;8(8):746-757.

S77. Chung YW, Yang HY, Kang SJ, Song EK, Seon JK. Allogeneic umbilical cord blood-derived mesenchymal stem cells combined with high tibial osteotomy: a retrospective study on safety and early results. Int Orthop. 2021;45(2):481-488.

S78. Ciemniewska-Gorzela K, Bąkowski P, Naczk J, Jakob R, Piontek T. Complex meniscus tears treated with collagen matrix wrapping and bone marrow blood injection: clinical effectiveness and survivorship after a minimum of 5 years' follow-up. Cartilage. 2020 Jun 1:1947603520924762.

S79. Colberg RE, Jurado Vélez JA, Walsh KP, Fleisig G. Short-term outcomes after pure bone marrow aspirate injection for severe knee osteoarthritis: a case series. Regen Med. 2020;15(7):1851-1859.

S80. Dallo I, Szwedowski D, Mobasheri A, Irlandini E, Gobbi A. A prospective study comparing leukocyte-poor platelet-rich plasma combined with hyaluronic acid and autologous microfragmented adipose tissue in patients with early knee osteoarthritis. Stem Cells Dev. 2021;30(13):651-659.

S81. Davatchi F, Abdollahi BS, Mohyeddin M, Shahram F, Nikbin B. Mesenchymal stem cell therapy for knee osteoarthritis. Preliminary report of four patients. Int J Rheum Dis. 2011;14(2):211-5.

S82. Davatchi F, Sadeghi Abdollahi B, Mohyeddin M, Nikbin B. Mesenchymal stem cell therapy for knee osteoarthritis: 5 years follow-up of three patients. Int J Rheum Dis. 2016;19(3):219-25.

S83. de Girolamo L, Schönhuber H, Viganò M, Bait C, Quaglia A, Thiebat G, Volpi P. Autologous matrix-induced chondrogenesis (AMIC) and AMIC enhanced by autologous concentrated bone marrow aspirate (BMAC) allow for stable clinical and functional improvements at up to 9 years follow-up: results from a randomized controlled study. J Clin Med. 2019;8(3):392.

S84. Dilogo IH, Canintika AF, Hanitya AL, Pawitan JA, Liem IK, Pandelaki J. Umbilical cord-derived mesenchymal stem cells for treating osteoarthritis of the knee: a single-arm, open-label study. Eur J Orthop Surg Traumatol. 2020;30(5):799-807.

S85. Dulic O, Lalic I, Kecojevic V, Gavrilovic G, Abazovic D, Miskulin M, Maric D, Bumbasirevic M. Do knee injection portals affect clinical results of bone marrow aspirate concentrate injection in the treatment of osteoarthritis? A prospective randomized controlled study. Regen Med. 2020;15(8):1987-2000.

S86. Ehlers CB, Webb AR, McCormick BP, Wyand TJ, Sarna N, Povey K, Marano G, Schainker L. Standardized platelet rich plasma injections for osteoarthritis of the knee. Cureus. 2020;12(10):S10900.

S87. Emadedin M, Aghdami N, Taghiyar L, Fazeli R, Moghadasali R, Jahangir S, Farjad R, Baghaban Eslaminejad M. Intra-articular injection of autologous mesenchymal stem cells in six patients with knee osteoarthritis. Arch Iran Med. 2012;15(7):422-8.

S88. Emadedin M, Ghorbani Liastani M, Fazeli R, Mohseni F, Moghadasali R, Mardpour S, Hosseini SE, Niknejadi M, Moeininia F, Aghahossein Fanni A, Baghban Eslaminejhad R, Vosough Dizaji A, Labibzadeh N, Mirazimi Bafghi A, Baharvand H, Aghdami N. Long-term follow-up of intraarticular injection of autologous mesenchymal stem cells in patients with knee, ankle, or hip osteoarthritis. Arch Iran Med. 2015;18(6):336-44.

S89. Estrada E, Décima JL, Rodríguez M, Di Tomaso M, Roberti J. Patient-reported outcomes after platelet-rich plasma, bone marrow aspirate, and adipose-derived mesenchymal stem cell injections for symptomatic knee osteoarthritis. Clin Med Insights Arthritis Musculoskelet Disord. 2020;13:1179544120931086.

S90. Fodor PB, Paulseth SG. Adipose derived stromal cell (ADSC) injections for pain management of osteoarthritis in the human knee joint. Aesthet Surg J. 2016;36(2):229-36. 
S91. Freitag J, Li D, Wickham J, Shah K, Tenen A. Effect of autologous adipose-derived mesenchymal stem cell therapy in the treatment of a post-traumatic chondral defect of the knee. BMJ Case Rep. 2017;2017:bcr2017220852.

S92. Freitag J, Norsworthy C, Wickham J, Shah K, Tenen A. High tibial osteotomy in combination with arthroscopic abrasion arthroplasty and autologous adipose-derived mesenchymal stem cell therapy in the treatment of advanced knee osteoarthritis. BMJ Case Rep. 2019;12(2):S228003.

S93. Freitag J, Shah K, Wickham J, Boyd R, Tenen A. The effect of autologous adipose derived mesenchymal stem cell therapy in the treatment of a large osteochondral defect of the knee following unsuccessful surgical intervention of osteochondritis dissecans - a case study. BMC Musculoskelet Disord. 2017;18(1):298.

S94. Freitag J, Shah K, Wickham J, Li D, Norsworthy C, Tenen A. Evaluation of autologous adiposederived mesenchymal stem cell therapy in focal chondral defects of the knee: a pilot case series. Regen Med. 2020;15(6):1703-1717.

S95. Freitag J, Wickham J, Shah K, Li D, Norsworthy C, Tenen A. Mesenchymal stem cell therapy combined with arthroscopic abrasion arthroplasty regenerates cartilage in patients with severe knee osteoarthritis: a case series. Regen Med. 2020;15(8):1957-1977.

S96. Gibbs N, Diamond R, Sekyere EO, Thomas WD. Management of knee osteoarthritis by combined stromal vascular fraction cell therapy, platelet-rich plasma, and musculoskeletal exercises: a case series. J Pain Res. 2015;8:799-806.

S97. Gobbi A, Chaurasia S, Karnatzikos G, Nakamura N. Matrix-induced autologous chondrocyte implantation versus multipotent stem cells for the treatment of large patellofemoral chondral lesions: a nonrandomized prospective trial. Cartilage. 2015;6(2):82-97.

S98. Gobbi A, Karnatzikos G, Sankineani SR. One-step surgery with multipotent stem cells for the treatment of large full-thickness chondral defects of the knee. Am J Sports Med. 2014;42(3):64857.

S99. Gobbi A, Whyte GP. Long-term clinical outcomes of one-stage cartilage repair in the knee with hyaluronic acid-based scaffold embedded with mesenchymal stem cells sourced from bone marrow aspirate concentrate. Am J Sports Med. 2019;47(7):1621-1628.

S100. Gobbi A, Whyte GP. One-stage cartilage repair using a hyaluronic acid-based scaffold with activated bone marrow-derived mesenchymal stem cells compared with microfracture: five-year follow-up. Am J Sports Med. 2016;44(11):2846-2854.

S101. Goncars V, Kalnberzs K, Jakobsons E, Enǵele I, Briede I, Blums K, Erglis K, Erglis M, Patetko L, Muiznieks I, Erglis A. Treatment of knee osteoarthritis with bone marrow-derived mononuclear cell injection: 12-month follow-up. Cartilage. 2019;10(1):26-35.

S102. Hauser RA, Orlofsky A. Regenerative injection therapy with whole bone marrow aspirate for degenerative joint disease: a case series. Clin Med Insights Arthritis Musculoskelet Disord. 2013;6:65-72.

S103. Heidari N, Borg TM, Olgiati S, Slevin M, Danovi A, Fish B, Wilson A, Noorani A. Microfragmented adipose tissue injection (mfat) may be a solution to the rationing of total knee replacement: a prospective, gender-bias mitigated, reproducible analysis at two years. Stem Cells Int. 2021;2021:9921015.

S104. Heidari N, Noorani A, Slevin M, Cullen A, Stark L, Olgiati S, Zerbi A, Wilson A. Patient-centered outcomes of microfragmented adipose tissue treatments of knee osteoarthritis: an observational, intention-to-treat study at twelve months. Stem Cells Int. 2020;2020:8881405.

S105. Hernigou P, Auregan JC, Dubory A, Flouzat-Lachaniette CH, Chevallier N, Rouard H. Subchondral stem cell therapy versus contralateral total knee arthroplasty for osteoarthritis following secondary osteonecrosis of the knee. Int Orthop. 2018;42(11):2563-2571.

S106. Hernigou P, Bouthors C, Bastard C, Flouzat Lachaniette CH, Rouard H, Dubory A. Subchondral bone or intra-articular injection of bone marrow concentrate mesenchymal stem cells in bilateral knee osteoarthritis: what better postpone knee arthroplasty at fifteen years? A randomized study. Int Orthop. 2021;45(2):391-399. 
S107. Hernigou P, Delambre J, Quiennec S, Poignard A. Human bone marrow mesenchymal stem cell injection in subchondral lesions of knee osteoarthritis: a prospective randomized study versus contralateral arthroplasty at a mean fifteen year follow-up. Int Orthop. 2021;45(2):365-373.

S108. Higuchi J, Yamagami R, Matsumoto T, Terao T, Inoue K, Tsuji S, Maenohara Y, Matsuzaki T, Chijimatsu R, Omata Y, Yano F, Tanaka S, Saito T. Associations of clinical outcomes and MRI findings in intra-articular administration of autologous adipose-derived stem cells for knee osteoarthritis. Regen Ther. 2020;14:332-340.

S109. Jiang Y, Cai Y, Zhang W, Yin Z, Hu C, Tong T, Lu P, Zhang S, Neculai D, Tuan RS, Ouyang HW. Human cartilage-derived progenitor cells from committed chondrocytes for efficient cartilage repair and regeneration. Stem Cells Transl Med. 2016;5(6):733-44.

S110. Kamei N, Ochi M, Adachi N, Ishikawa M, Yanada S, Levin LS, Kamei G, Kobayashi T. The safety and efficacy of magnetic targeting using autologous mesenchymal stem cells for cartilage repair. Knee Surg Sports Traumatol Arthrosc. 2018;26(12):3626-3635.

S111. Kasemkijwattana C, Hongeng S, Kesprayura S, Rungsinaporn V, Chaipinyo K, Chansiri K. Autologous bone marrow mesenchymal stem cells implantation for cartilage defects: two cases report. J Med Assoc Thai. 2011;94(3):395-400.

S112. Kazemian G, Manafi Rasi A, Baroutkoub M, Tavakoli Darestani R. Effect of administration of mesenchymal stem cells on cartilage recovery and knee function in patients with knee osteoarthritis. Med Sci. 2020;24(103):1019-1026.

S113. Kim GB, Kim J-D, Choi Y, Choi CH, Lee GW. Intra-articular bone marrow aspirate concentrate injection in patients with knee osteoarthritis. Appl Sci. 2020;10(17):5945.

S114. Kim YS, Choi YJ, Koh YG. Mesenchymal stem cell implantation in knee osteoarthritis: an assessment of the factors influencing clinical outcomes. Am J Sports Med. 2015;43(9):2293-301.

S115. Kim YS, Choi YJ, Lee SW, Kwon OR, Suh DS, Heo DB, Koh YG. Assessment of clinical and MRI outcomes after mesenchymal stem cell implantation in patients with knee osteoarthritis: a prospective study. Osteoarthritis Cartilage. 2016;24(2):237-45.

S116. Kim YS, Choi YJ, Suh DS, Heo DB, Kim YI, Ryu JS, Koh YG. Mesenchymal stem cell implantation in osteoarthritic knees: is fibrin glue effective as a scaffold? Am J Sports Med. 2015;43(1):176-85.

S117. Kim YS, Chung PK, Suh DS, Heo DB, Tak DH, Koh YG. Implantation of mesenchymal stem cells in combination with allogenic cartilage improves cartilage regeneration and clinical outcomes in patients with concomitant high tibial osteotomy. Knee Surg Sports Traumatol Arthrosc. 2020;28(2):544-554.

S118. Kim YS, Koh YG. Comparative matched-pair analysis of open-wedge high tibial osteotomy with versus without an injection of adipose-derived mesenchymal stem cells for varus knee osteoarthritis: clinical and second-look arthroscopic results. Am J Sports Med. 2018;46(11):26692677.

S119. Kim YS, Kwon OR, Choi YJ, Suh DS, Heo DB, Koh YG. Comparative matched-pair analysis of the injection versus implantation of mesenchymal stem cells for knee osteoarthritis. Am J Sports Med. 2015;43(11):2738-46.

S120. Kim YS, Suh DS, Tak DH, Chung PK, Koh YG. Mesenchymal stem cell implantation in knee osteoarthritis: midterm outcomes and survival analysis in 467 patients. Orthop J Sports Med. 2020;8(12):2325967120969189.

S121. Kim YS, Suh DS, Tak DH, Chung PK, Kwon YB, Kim TY, Koh YG. Comparative matched-pair cohort analysis of the short-term clinical outcomes of mesenchymal stem cells versus hyaluronic acid treatments through intra-articular injections for knee osteoarthritis. J Exp Orthop. 2020;7(1):90.

S122. Kim YS, Suh DS, Tak DH, Chung PK, Kwon YB, Kim TY, Koh YG. Factors influencing clinical and mri outcomes of mesenchymal stem cell implantation with concomitant high tibial osteotomy for varus knee osteoarthritis. Orthop J Sports Med. 2021;9(2):2325967120979987.

S123. Koh YG, Choi YJ, Kwon OR, Kim YS. Second-look arthroscopic evaluation of cartilage lesions 
after mesenchymal stem cell implantation in osteoarthritic knees. Am J Sports Med. 2014;42(7):1628-37.

S124. Koh YG, Choi YJ, Kwon SK, Kim YS, Yeo JE. Clinical results and second-look arthroscopic findings after treatment with adipose-derived stem cells for knee osteoarthritis. Knee Surg Sports Traumatol Arthrosc. 2015;23(5):1308-16.

S125. Koh YG, Kwon OR, Kim YS, Choi YJ, Tak DH. Adipose-derived mesenchymal stem cells with microfracture versus microfracture alone: 2-year follow-up of a prospective randomized trial. Arthroscopy. 2016;32(1):97-109.

S126. Kuroda R, Ishida K, Matsumoto T, Akisue T, Fujioka H, Mizuno K, Ohgushi H, Wakitani S, Kurosaka M. Treatment of a full-thickness articular cartilage defect in the femoral condyle of an athlete with autologous bone-marrow stromal cells. Osteoarthritis Cartilage. 2007;15(2):226-31.

S127. Lamo-Espinosa JM, Blanco JF, Sánchez M, Moreno V, Granero-Moltó F, Sánchez-Guijo F, Crespo-Cullel Í, Mora G, San Vicente DD, Pompei-Fernández O, Aquerreta JD, Núñez-Córdoba JM, Vitoria Sola M, Valentí-Azcárate A, Andreu EJ, Del Consuelo Del Cañizo M, Valentí-Nin JR, Prósper F. Phase II multicenter randomized controlled clinical trial on the efficacy of intra-articular injection of autologous bone marrow mesenchymal stem cells with platelet rich plasma for the treatment of knee osteoarthritis. J Transl Med. 2020;18(1):356.

S128. Lamo-Espinosa JM, Mora G, Blanco JF, Granero-Moltó F, Núñez-Córdoba JM, López-Elío S, Andreu E, Sánchez-Guijo F, Aquerreta JD, Bondía JM, Valentí-Azcárate A, Del Consuelo Del Cañizo M, Villarón EM, Valentí-Nin JR, Prósper F. Intra-articular injection of two different doses of autologous bone marrow mesenchymal stem cells versus hyaluronic acid in the treatment of knee osteoarthritis: long-term follow up of a multicenter randomized controlled clinical trial (phase I/II). J Transl Med. 2018;16(1):213.

S129. Lapuente JP, Dos-Anjos S, Blázquez-Martínez A. Intra-articular infiltration of adipose-derived stromal vascular fraction cells slows the clinical progression of moderate-severe knee osteoarthritis: hypothesis on the regulatory role of intra-articular adipose tissue. J Orthop Surg Res. 2020;15(1): 137.

S130. Lee NH, Na SM, Ahn HW, Kang JK, Seon JK, Song EK. Allogenic human umbilical cord bloodderived mesenchymal stem cells are more effective than bone marrow aspiration concentrate for cartilage regeneration after high tibial osteotomy in medial unicompartmental osteoarthritis of knee. Arthroscopy. 2021;37(8):2521-2530.

S131. Leigheb M, Bosetti M, De Consoli A, Borrone A, Cannas M, Grassi F. Chondral tissue engineering of the reumatoid knee with collagen matrix autologous chondrocytes implant. Acta Biomed. 2017;88(4S):107-113.

S132. Li J, Shao Q, Zhu X, Sun G. Efficacy of autologous bone marrow mesenchymal stem cells in the treatment of knee osteoarthritis and their effects on the expression of serum TNF- $\alpha$ and IL- 6 . J Musculoskelet Neuronal Interact. 2020;20(1):128-135.

S133. Lim HC, Park YB, Ha CW, Cole BJ, Lee BK, Jeong HJ, Kim MK, Bin SI, Choi CH, Choi CH, Yoo JD; Cartistem Research Group, Yoon JR, Chung JY. Allogeneic umbilical cord blood-derived mesenchymal stem cell implantation versus microfracture for large, full-thickness cartilage defects in older patients: a multicenter randomized clinical trial and extended 5-year clinical follow-up. Orthop J Sports Med. 2021;9(1):2325967120973052.

S134. Liu HC, Liu TT, Liu YL, Wang JH, Chang CH, Shih TT, Lin FH. Atelocollagen-embedded chondrocyte precursors as a treatment for grade- 4 cartilage defects of the femoral condyle: a case series with up to 9-year follow-up. Biomolecules. 2021;11(7):942.

S135. Lu L, Dai C, Du H, Li S, Ye P, Zhang L, Wang X, Song Y, Togashi R, Vangsness CT, Bao C. Intra-articular injections of allogeneic human adipose-derived mesenchymal progenitor cells in patients with symptomatic bilateral knee osteoarthritis: a Phase I pilot study. Regen Med. 2020;15(5):1625-1636.

S136. Magnanelli S, Screpis D, Di Benedetto P, Natali S, Causero A, Zorzi C. Open-wedge high tibial osteotomy associated with Lipogems ${ }^{\circledR}$ intra-articular injection for the treatment of varus knee 
osteoarthritis - retrospective study. Acta Biomed. 2020;91(14-S):S2020022.

S137. Malanga GA, Chirichella PS, Hogaboom NS, Capella T. Clinical evaluation of micro-fragmented adipose tissue as a treatment option for patients with meniscus tears with osteoarthritis: a prospective pilot study. Int Orthop. 2021;45(2):473-480.

S138. Martinčič D, Leban J, Filardo G, Busacca M, Barlič A, Veber M, Drobnič M. Autologous chondrocytes versus filtered bone marrow mesenchymal stem/stromal cells for knee cartilage repair-a prospective study. Int Orthop. 2020.

S139. Mehling B, Hric M, Salatkova A, Vetrak R, Santora D, Ovariova M, Mihalyova R, Manvelyan M. A Retrospective study of stromal vascular fraction cell therapy for osteoarthritis. J Clin Med Res. 2020;12(11):747-751.

S140. Mehrabani D, Mojtahed Jaberi F, Zakerinia M, Hadianfard MJ, Jalli R, Tanideh N, Zare S. The healing effect of bone marrow-derived stem cells in knee osteoarthritis: a case report. World J Plast Surg. 2016;5(2):168-74.

S141. Monckeberg JE, Rafols C, Apablaza F, Gerhard P, Rosales J. Intra-articular administration of peripheral blood stem cells with platelet-rich plasma regenerated articular cartilage and improved clinical outcomes for knee chondral lesions. Knee 2019;26(4):824-31.

S142. Olivos-Meza A, Pérez Jiménez FJ, Granados-Montiel J, Landa-Solís C, Cortés González S, Jiménez Aroche CA, Valdez Chávez M, Renán León S, Gomez-Garcia R, Martínez-López V, Ortega-Sánchez C, Parra-Cid C, Velasquillo Martinez C, Ibarra C. First clinical application of polyurethane meniscal scaffolds with mesenchymal stem cells and assessment of cartilage quality with $\mathrm{t} 2$ mapping at 12 months. Cartilage. 2019:1947603519852415.

S143. Onoi Y, Hiranaka T, Nishida R, Takase K, Fujita M, Hida Y, Fujishiro T, Okamoto K. Secondlook arthroscopic findings of cartilage and meniscus repair after injection of adipose-derived regenerative cells in knee osteoarthrits: Report of two cases. Regen Ther. 2019;11:212-216.

S144. Orozco L, Munar A, Soler R, Alberca M, Soler F, Huguet M, Sentís J, Sánchez A, García-Sancho J. Treatment of knee osteoarthritis with autologous mesenchymal stem cells: two-year follow-up results. Transplantation. 2014;97(11):S66-8.

S145. Pak J, Lee JH, Park KS, Jeong BC, Lee SH. Regeneration of cartilage in human knee osteoarthritis with autologous adipose tissue-derived stem cells and autologous extracellular matrix. Biores Open Access. 2016;5(1):192-200.

S146. Pak J. Regeneration of human bones in hip osteonecrosis and human cartilage in knee osteoarthritis with autologous adipose-tissue-derived stem cells: a case series. J Med Case Rep. 2011;5:296.

S147. Papalia R, Zampogna B, Russo F, Vasta S, Campi S, Saccone L, Di Giacomo G, Vadalà G, Denaro V. Adipose-derived stromal vascular fraction processed with different systems for the treatment of knee osteoarthritis: a pilot study on cell proliferation and clinical results. J Biol Regul Homeost Agents. 2020;34(5 Suppl. 1):113-119.

S148. Park YB, Ha CW, Lee CH, Yoon YC, Park YG. Cartilage regeneration in osteoarthritic patients by a composite of allogeneic umbilical cord blood-derived mesenchymal stem cells and hyaluronate hydrogel: results from a clinical trial for safety and proof-of-concept with 7 years of extended follow-up. Stem Cells Transl Med. 2017;6(2):613-621.

S149. Peretti GM, Ulivi M, De Girolamo L, Meroni V, Lombardo MD, Mangiavini L. Evaluation of the use of autologous micro-fragmented adipose tissue in the treatment of knee osteoarthritis: preliminary results of a randomized controlled trial. J Biol Regul Homeost Agents. 2018;32(6 Suppl. 1):193-199.

S150. Pintat J, Silvestre A, Magalon G, Gadeau AP, Pesquer L, Perozziello A, Peuchant A, Mounayer C, Dallaudière B. Intra-articular injection of mesenchymal stem cells and platelet-rich plasma to treat patellofemoral osteoarthritis: preliminary results of a long-term pilot study. J Vasc Interv Radiol. 2017;28(12):1708-1713.

S151. Prodromos C, Finkle S. Autologous biologic treatment with fat, bone marrow aspirate and platelet rich plasma is an effective alternative to total knee arthroplasty for patients with moderate knee arthrosis. Medicines (Basel). 2020;7(6):37. 
S152. Qiao Z, Tang J, Yue B, Wang J, Zhang J, Xuan L, Dai C, Li S, Li M, Xu C, Dai K, Wang Y. Human adipose-derived mesenchymal progenitor cells plus microfracture and hyaluronic acid for cartilage repair: a Phase IIa trial. Regen Med. 2020 Jan;15(1):1193-1214.

S153. Rodriguez-Fontan F, Piuzzi NS, Kraeutler MJ, Pascual-Garrido C. Early clinical outcomes of intraarticular injections of bone marrow aspirate concentrate for the treatment of early osteoarthritis of the hip and knee: a cohort study. PM R. 2018;10(12):1353-1359.

S154. Ruane JJ, Ross A, Zigmont V, McClure D, Gascon G. A single-blinded randomized controlled trial of mesenchymal stem cell therapy for the treatment of osteoarthritis of the knee with active control. J Stem Cells Regen Med. 2021;17(1):3-17.

S155. Russo A, Condello V, Madonna V, Guerriero M, Zorzi C. Autologous and micro-fragmented adipose tissue for the treatment of diffuse degenerative knee osteoarthritis. J Exp Orthop. 2017;4(1):33.

S156. Russo A, Screpis D, Di Donato SL, Bonetti S, Piovan G, Zorzi C. Autologous micro-fragmented adipose tissue for the treatment of diffuse degenerative knee osteoarthritis: an update at 3 year follow-up. J Exp Orthop. 2018;5(1):52.

S157. Ryu DJ, Jeon YS, Park JS, Bae GC, Kim JS, Kim MK. Comparison of bone marrow aspirate concentrate and allogenic human umbilical cord blood derived mesenchymal stem cell implantation on chondral defect of knee: assessment of clinical and magnetic resonance imaging outcomes at 2year follow-up. Cell Transplant. 2020 Jan-Dec;29:963689720943581.

S158. Sadlik B, Jaroslawski G, Gladysz D, Puszkarz M, Markowska M, Pawelec K, Boruczkowski D, Oldak T. Knee cartilage regeneration with umbilical cord mesenchymal stem cells embedded in collagen scaffold using dry arthroscopy technique. Adv Exp Med Biol. 2017;1020:113-122.

S159. Sampson S, Smith J, Vincent H, Aufiero D, Zall M, Botto-van-Bemden A. Intra-articular bone marrow concentrate injection protocol: short-term efficacy in osteoarthritis. Regen Med. 2016;11(6):511-520.

S160. Santoprete S, Marchetti F, Rubino C, Bedini MG, Nasto LA, Cipolloni V, Pola E. Fresh autologous stromal tissue fraction for the treatment of knee osteoarthritis related pain and disability. Orthop Rev (Pavia). 2021;13(1):9161.

S161. Saris TFF, de Windt TS, Kester EC, Vonk LA, Custers RJH, Saris DBF. Five-year outcome of 1stage cell-based cartilage repair using recycled autologous chondrons and allogenic mesenchymal stromal cells: a first-in-human clinical trial. Am J Sports Med. 2021;49(4):941-947.

S162. Saw KY, Anz AW, Ng RC, Jee CS, Low SF, Dorvault C, Johnson KB. Arthroscopic subchondral drilling followed by injection of peripheral blood stem cells and hyaluronic acid showed improved outcome compared to hyaluronic acid and physiotherapy for massive knee chondral defects: a randomized controlled trial. Arthroscopy. 2021;37(8):2502-2517.

S163. Sekiya I, Katano H, Mizuno M, Koga H, Masumoto J, Tomita M, Ozeki N. Alterations in cartilage quantification before and after injections of mesenchymal stem cells into osteoarthritic knees. Sci Rep. 2021;11(1):13832.

S164. Sekiya I, Koga H, Otabe K, Nakagawa Y, Katano H, Ozeki N, Mizuno M, Horie M, Kohno Y, Katagiri K, Watanabe N, Muneta T. Additional use of synovial mesenchymal stem cell transplantation following surgical repair of a complex degenerative tear of the medial meniscus of the knee: a case report. Cell Transplant. 2019;28(11):1445-1454.

S165. Shadmanfar S, Labibzadeh N, Emadedin M, Jaroughi N, Azimian V, Mardpour S, Kakroodi FA, Bolurieh T, Hosseini SE, Chehrazi M, Niknejadi M, Baharvand H, Gharibdoost F, Aghdami N. Intra-articular knee implantation of autologous bone marrow-derived mesenchymal stromal cells in rheumatoid arthritis patients with knee involvement: results of a randomized, triple-blind, placebo-controlled phase 1/2 clinical trial. Cytotherapy. 2018;20(4):499-506.

S166. Shetty AA, Kim SJ, Ahmed S, Trattnig S, Kim SA, Jang HJ. A cost-effective cell- and matrixbased minimally invasive single-stage chondroregenerative technique developed with validated vertical translation methodology. Ann R Coll Surg Engl. 2018;100(3):240-246.

S167. Shimomura K, Yasui Y, Koizumi K, Chijimatsu R, Hart DA, Yonetani Y, Ando W, Nishii T, 
Kanamoto T, Horibe S, Yoshikawa H, Nakamura N, Sakaue M, Sugita N, Moriguchi Y. First-inhuman pilot study of implantation of a scaffold-free tissue-engineered construct generated from autologous synovial mesenchymal stem cells for repair of knee chondral lesions. Am J Sports Med. 2018;46(10):2384-2393.

S168. Simunec D, Salari H, Meyer J. Treatment of Grade 3 and 4 Osteoarthritis with intraoperatively separated adipose tissue-derived stromal vascular fraction: a comparative case series. Cells. 2020;9(9):S2096.

S169. Skowroński J, Skowroński R, Rutka M. Cartilage lesions of the knee treated with blood mesenchymal stem cells - results. Ortop Traumatol Rehabil. 2012;14(6):569-77.

S170. Smyshlyaev IA, Gilfanov SI, Kopylov VA, Gilmutdinov RG, Pulin II, Korsakov IN, Gilmutdinova IR, Petrikina AP, Eremin PS, Kruchkova OV, Abeltsev VP, Zagorodniy NV, Zorin VL, Vasilyev VS, Pupynin DY, Eremin II. Safety and effectiveness of intraarticular administration of adiposederived stromal vascular fraction for treatment of knee articular cartilage degenerative damage: preliminary results of a clinical trial. Travmatol Ortoped Rossii 2017;23:17-31.

S171. Song JS, Hong KT, Kim NM, Jung JY, Park HS, Chun YS, Kim SJ. Cartilage regeneration in osteoarthritic knees treated with distal femoral osteotomy and intra-lesional implantation of allogenic human umbilical cord blood-derived mesenchymal stem cells: A report of two cases. Knee. 2019;26(6):1445-1450.

S172. Song JS, Hong KT, Kim NM, Jung JY, Park HS, Lee SH, Cho YJ, Kim SJ. Implantation of allogenic umbilical cord blood-derived mesenchymal stem cells improves knee osteoarthritis outcomes: Two-year follow-up. Regen Ther. 2020 Jan 14;14:32-39.

S173. Song JS, Hong KT, Kim NM, Park HS, Choi NH. Human umbilical cord blood-derived mesenchymal stem cell implantation for osteoarthritis of the knee. Arch Orthop Trauma Surg. 2020;140(4):503-509.

S174. Song JS, Hong KT, Kong CG, Kim NM, Jung JY, Park HS, Kim YJ, Chang KB, Kim SJ. High tibial osteotomy with human umbilical cord blood-derived mesenchymal stem cells implantation for knee cartilage regeneration. World J Stem Cells. 2020;12(6):514-526.

S175. Srinivas PL, Kumar PP. Role of PRP and stem cell injections in osteoarthritic patients of knee joint. J Evol Med Dent Sci 2015;4(54):9468-9474.

S176. Toan DD, Binh NT, Dung TT, Thuy LQ, Hoa ND, Long NH, Tung PS. The effectiveness of knee osteoarthritis treatment by arthroscopic microfracture technique in combination with autologous bone marrow stem cells transplantation. J Back Musculoskelet Rehabil. 2020;33(3):397-403.

S177. Turajane T, Chaveewanakorn U, Fongsarun W, Aojanepong J, Papadopoulos KI. Avoidance of total knee arthroplasty in early osteoarthritis of the knee with intra-articular implantation of autologous activated peripheral blood stem cells versus hyaluronic acid: a randomized controlled trial with differential effects of growth factor addition. Stem Cells Int. 2017;2017:8925132.

S178. Turajane T, Chaweewannakorn U, Larbpaiboonpong V, Aojanepong J, Thitiset T, Honsawek S, Fongsarun J, Papadopoulos KI. Combination of intra-articular autologous activated peripheral blood stem cells with growth factor addition/ preservation and hyaluronic acid in conjunction with arthroscopic microdrilling mesenchymal cell stimulation Improves quality of life and regenerates articular cartilage in early osteoarthritic knee disease. J Med Assoc Thai. 2013;96(5):580-588.

S179. Van Genechten W, Vuylsteke K, Martinez PR, Swinnen L, Sas K, Verdonk P. Autologous microfragmented adipose tissue (MFAT) to treat symptomatic knee osteoarthritis: early outcomes of a consecutive case series. J Clin Med. 2021;10(11):2231.

S180. Varady NH, Cate G, Barghi A, Jobe N, Yakin D, Ylanan RC, Arnold CA. Positive early clinical outcomes of bone marrow aspirate concentrate for osteoarthritis using a novel fenestrated trocar. Knee. 2020;27(5):1627-1634.

S181. Veber M, Vogler J, Knežević M, Barlič A, Drobnič M. Combination of filtered bone marrow aspirate and biomimetic scaffold for the treatment of knee osteochondral lesions: cellular and early clinical results of a single centre case series. Tissue Eng Regen Med. 2020;17(3):375-386.

S182. Wakitani S, Imoto K, Yamamoto T, Saito M, Murata N, Yoneda M. Human autologous culture 
expanded bone marrow mesenchymal cell transplantation for repair of cartilage defects in osteoarthritic knees. Osteoarthritis Cartilage. 2002;10(3):199-206.

S183. Wang J, Wright KT, Perry J, Tins B, Hopkins T, Hulme C, McCarthy HS, Brown A, Richardson JB. Combined autologous chondrocyte and bone marrow mesenchymal stromal cell implantation in the knee: an 8-year follow up of two first-in-man cases. Cell Transplant. 2019;28(7):924-931.

S184. Wells K, Klein M, Hurwitz N, Santiago K, Cheng J, Abutalib Z, Beatty N, Lutz G. Cellular and clinical analyses of autologous bone marrow aspirate injectate for knee osteoarthritis: a pilot study. PM R. 2020 Jun 5. Epub ahead of print.

S185. Whitehouse MR, Howells NR, Parry MC, Austin E, Kafienah W, Brady K, Goodship AE, Eldridge JD, Blom AW, Hollander AP. Repair of torn avascular meniscal cartilage using undifferentiated autologous mesenchymal stem cells: from in vitro optimization to a first-in-human study. Stem Cells Transl Med. 2017;6(4):1237-1248.

S186. Zhang Z, Zhong X, Ji H, Tang Z, Bai J, Yao M, Hou J, Zheng M, Wood DJ, Sun J, Zhou SF, Liu A. Matrix-induced autologous chondrocyte implantation for the treatment of chondral defects of the knees in Chinese patients. Drug Des Devel Ther. 2014;8:2439-48.

S187. Zhao X, Ruan J, Tang H, Li J, Shi Y, Li M, Li S, Xu C, Lu Q, Dai C. Multi-compositional MRI evaluation of repair cartilage in knee osteoarthritis with treatment of allogeneic human adiposederived mesenchymal progenitor cells. Stem Cell Res Ther. 2019;10(1):308. 